Supporting Information for

\title{
Masked Radicals: Iron Complexes of Trityl, Benzophenone, and Phenylacetylene
}

K. Cory MacLeod, ${ }^{\ddagger}$ Ida M. DiMucci, Edward P. Zovinka,, Sean F. McWilliams, Brandon Q. Mercado, Kyle M. Lancaster,* and Patrick L. Holland*

Table of Contents

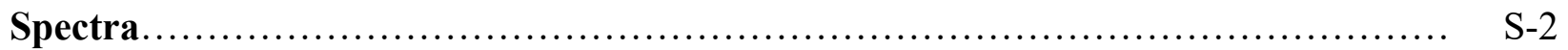

Mössbauer Spectra................................................ S-2

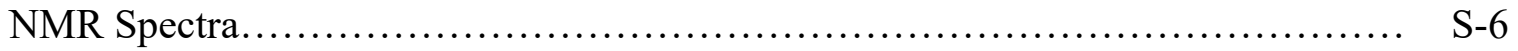

UV-visible Spectra.................................................. S-10

IR Spectra........................................................ S-15

X-Ray Absorption Spectroscopy Details................................ S-19

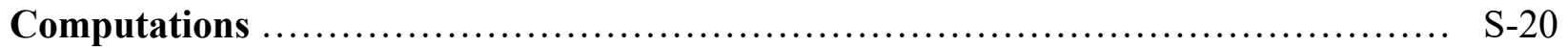

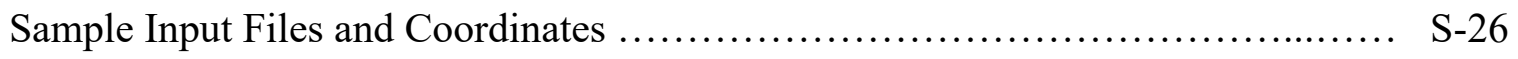

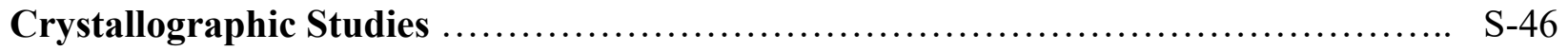

References............................................................. S-195 


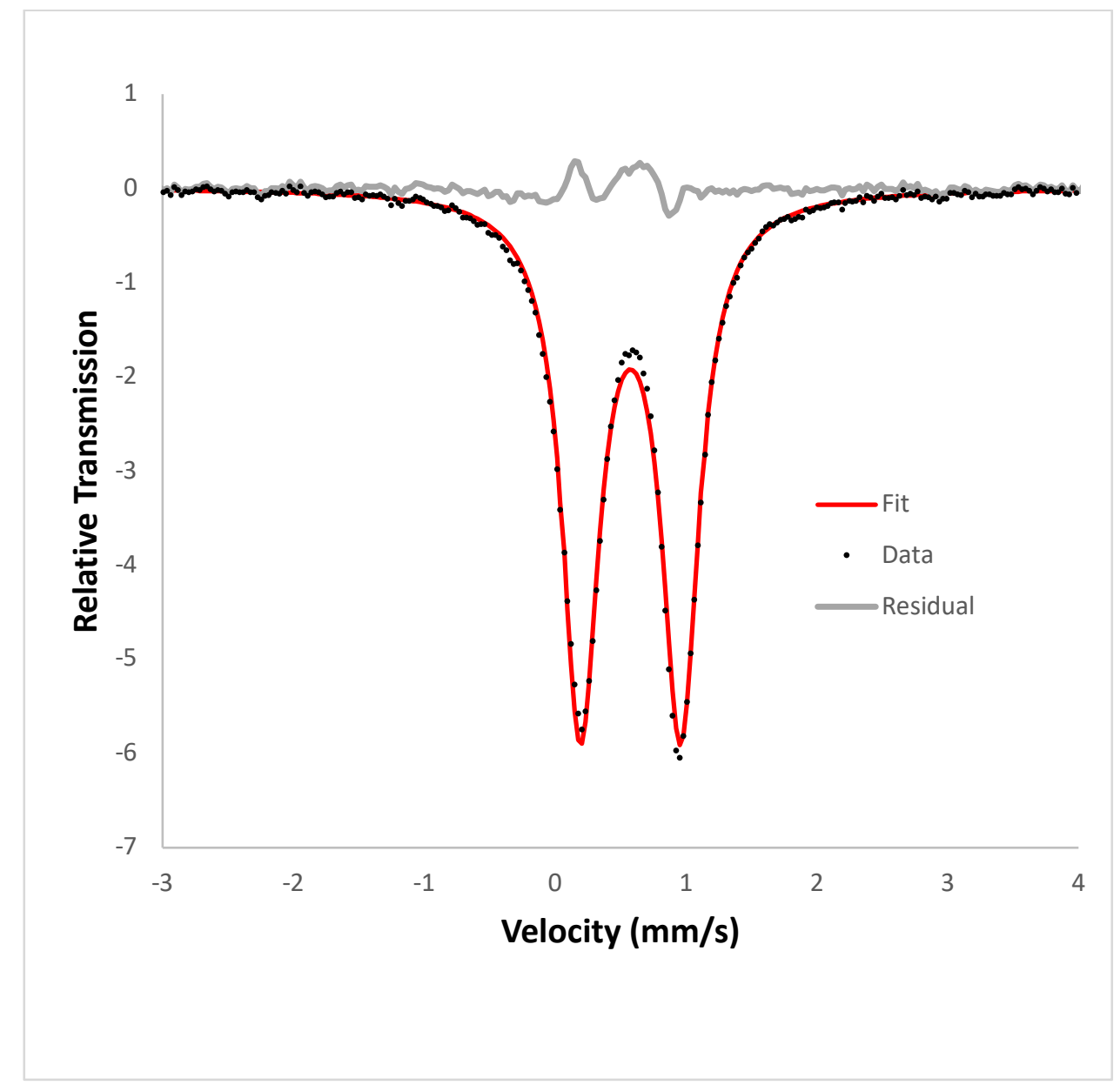

Figure S-1. Zero-field Mössbauer spectrum of $\mathrm{L}^{\mathrm{Me}} \mathrm{Fe}\left(\mathrm{CPh}_{3}\right)$ (1) recorded at $80 \mathrm{~K}$. The black circles are the data, the red line is a one-component simulation of the data $\left(\delta=0.58 \mathrm{~mm} / \mathrm{s},\left|\Delta E_{\mathrm{Q}}\right|=0.76\right.$ $\mathrm{mm} / \mathrm{s}, \Gamma=0.35 \mathrm{~mm} \mathrm{~s}^{-1}$ ), and the gray line is the residual. 

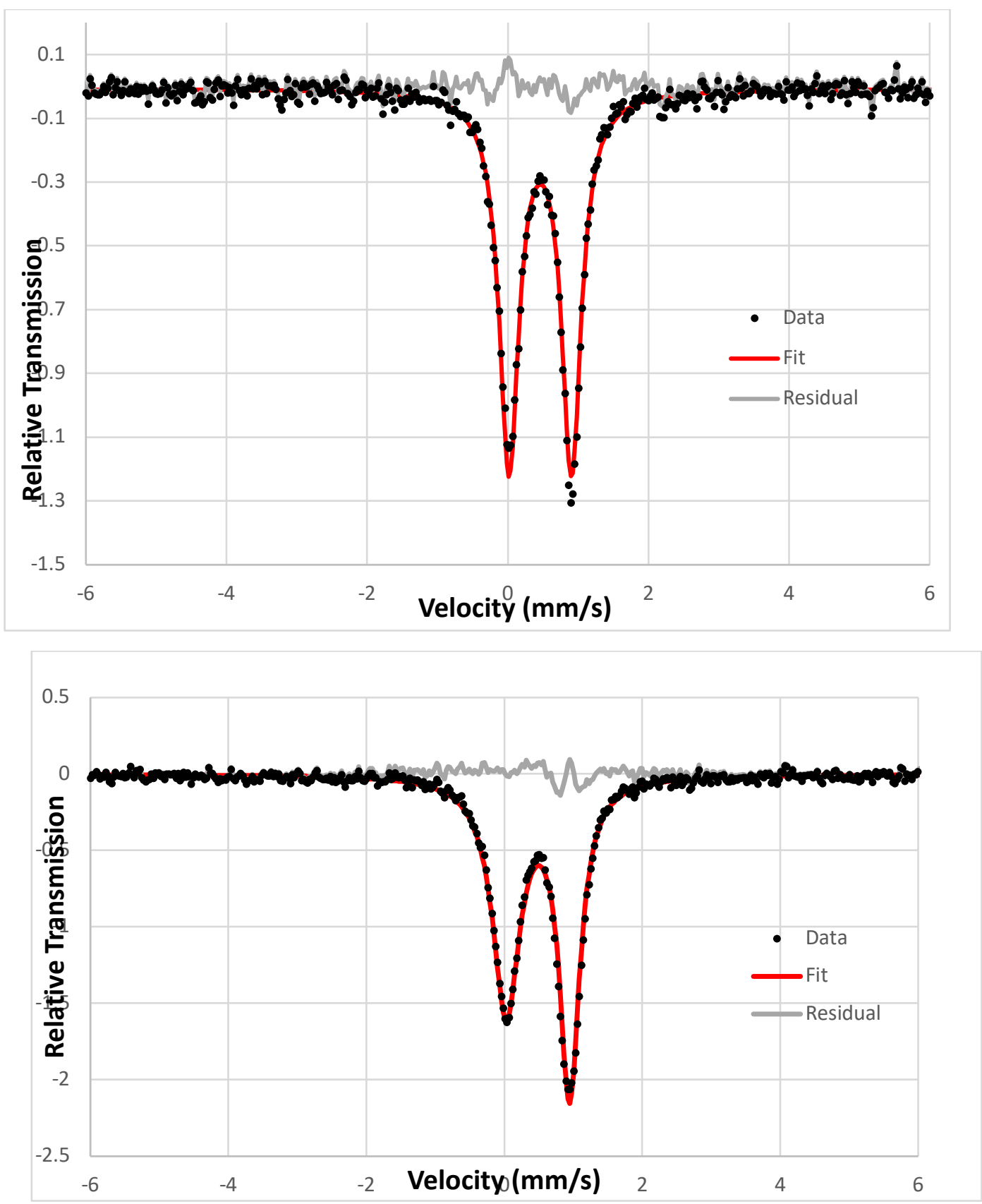

Figure S-2. Zero-field Mössbauer spectrum of $\mathrm{L}^{\mathrm{Me}} \mathrm{Fe}\left(\mathrm{Ph}_{2} \mathrm{CO}\right)$ (2) recorded at (a) $223 \mathrm{~K}$ and (b) $173 \mathrm{~K}$. The black circles are the data, the red lines are one-component simulations of the data and the gray lines are the residuals. Fit parameters: (a, $223 \mathrm{~K}$ ) $\delta=0.46 \mathrm{~mm} / \mathrm{s},\left|\Delta E_{\mathrm{Q}}\right|=0.89 \mathrm{~mm} / \mathrm{s}, \Gamma=$ $0.34 \mathrm{~mm} / \mathrm{s} ;(\mathrm{b}, 173 \mathrm{~K}) \delta=0.49 \mathrm{~mm} / \mathrm{s},\left|\Delta E_{\mathrm{Q}}\right|=0.92 \mathrm{~mm} / \mathrm{s}, \Gamma_{\mathrm{L}}=0.48 \mathrm{~mm} / \mathrm{s}, \Gamma_{\mathrm{R}}=0.36 \mathrm{~mm} / \mathrm{s}$. 


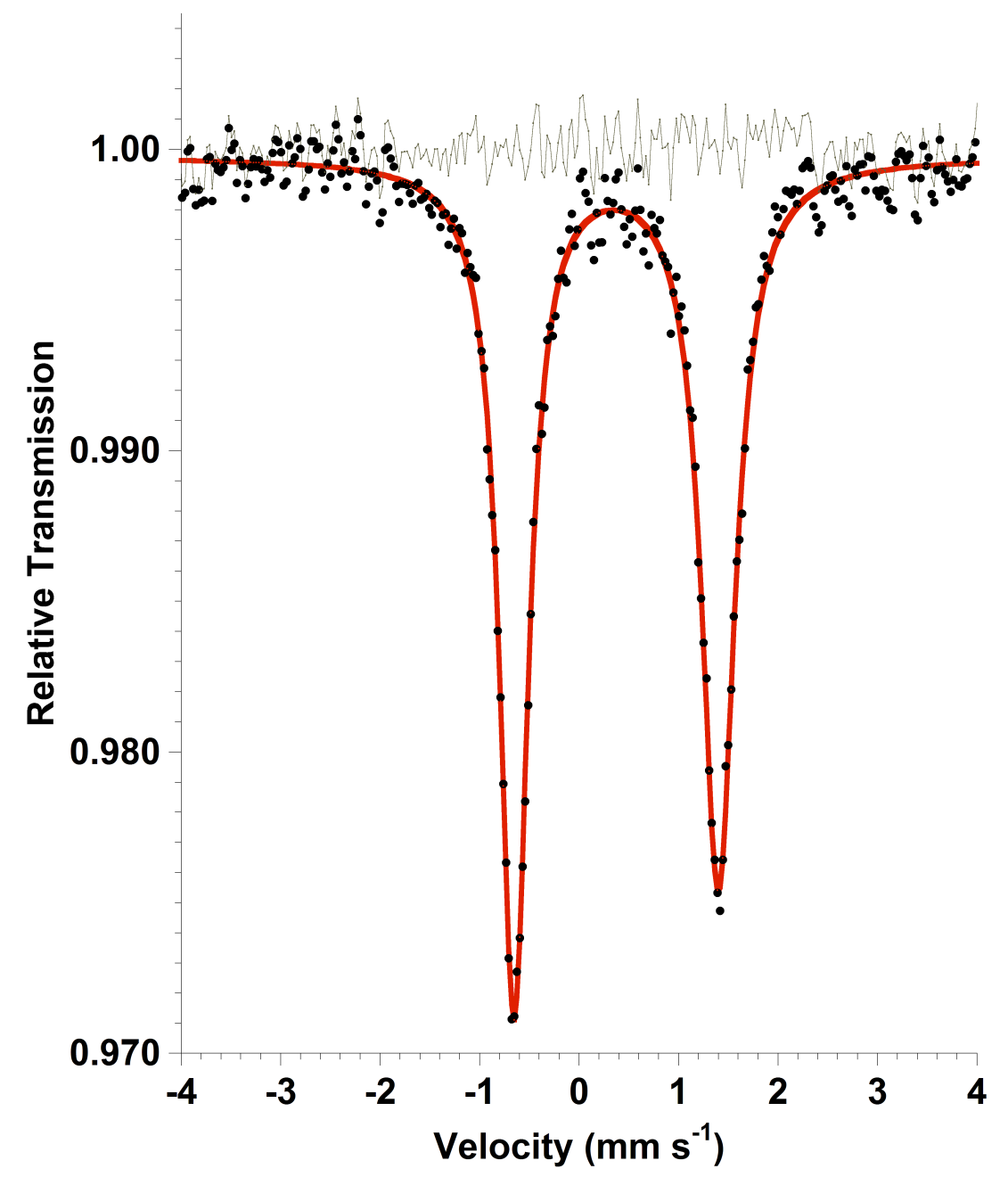

Figure S-3. Zero-field Mössbauer spectrum of [K(2.2.2-cryptand) $]\left[\mathrm{L}^{\mathrm{Me}} \mathrm{FeCH}_{3}\right]$ (3) recorded at 80 $\mathrm{K}$. The black circles are the data, the red line is a one-component simulation of the data $(\delta=0.38$ $\mathrm{mm} / \mathrm{s},\left|\Delta E_{\mathrm{Q}}\right|=2.06 \mathrm{~mm} / \mathrm{s}, \Gamma_{\mathrm{L}}=0.35 \mathrm{~mm} / \mathrm{s}, \Gamma_{\mathrm{R}}=0.42 \mathrm{~mm} / \mathrm{s}$.), and the gray line is the residual. 


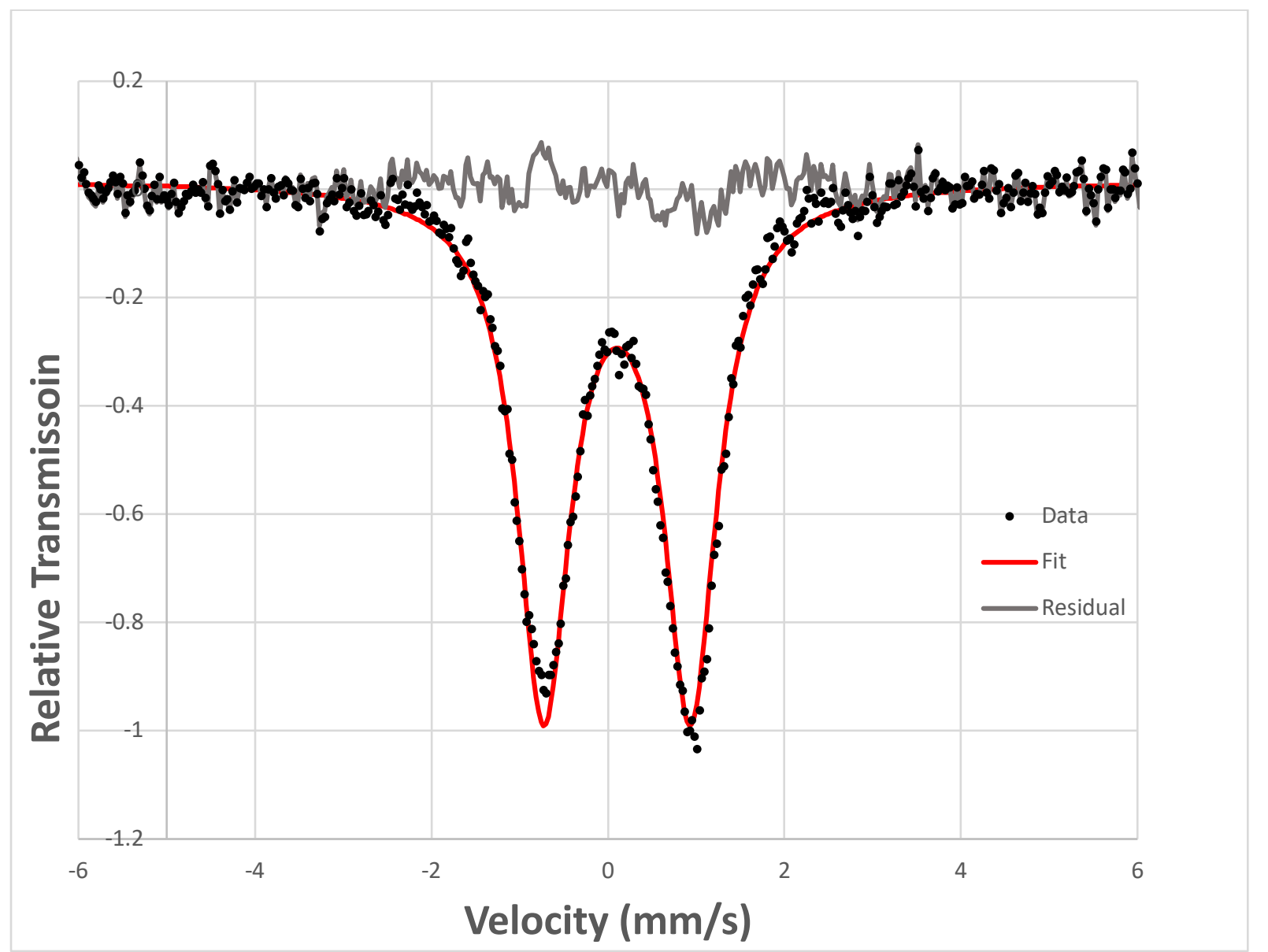

Figure S-4. Zero-field Mössbauer spectrum of $\mathrm{L}^{\mathrm{Me}} \mathrm{Fe}\left(\mathrm{OCPh}_{2} \mathrm{CHCPh}\right)(6)$ recorded at $173 \mathrm{~K}$. The black circles are the data, the red line is a one-component simulation of the data $(\delta=0.10 \mathrm{~mm} / \mathrm{s}$, $\left.\left|\Delta E_{\mathrm{Q}}\right|=1.66 \mathrm{~mm} / \mathrm{s}, \Gamma=0.73 \mathrm{~mm} / \mathrm{s}\right)$, and the gray line is the residual. 


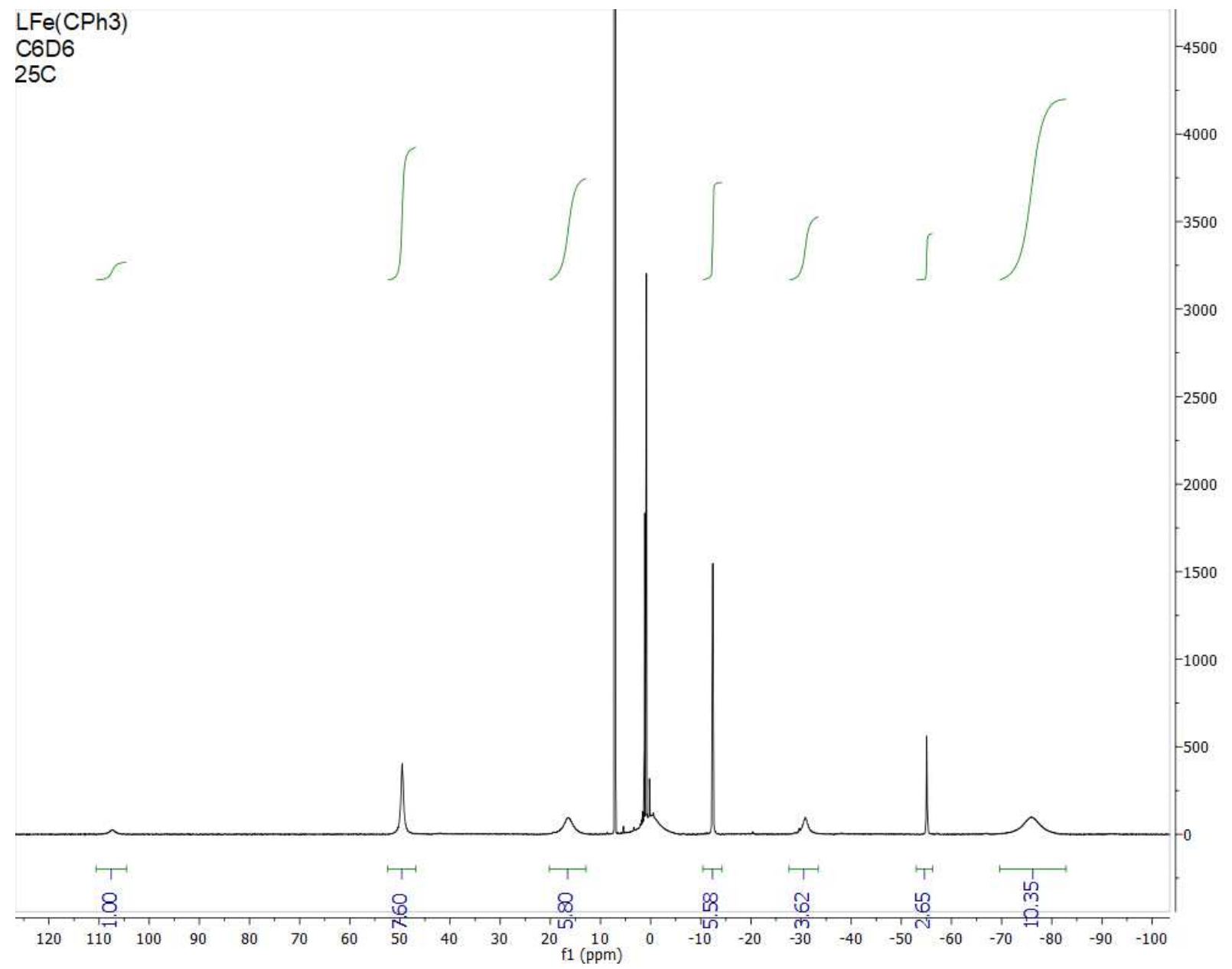

Figure S-5: ${ }^{1} \mathrm{H}$ NMR spectrum of $\mathrm{L}^{\mathrm{Me}} \mathrm{Fe}\left(\mathrm{CPh}_{3}\right)$ (1) in $\mathrm{C}_{6} \mathrm{D}_{6}$. 


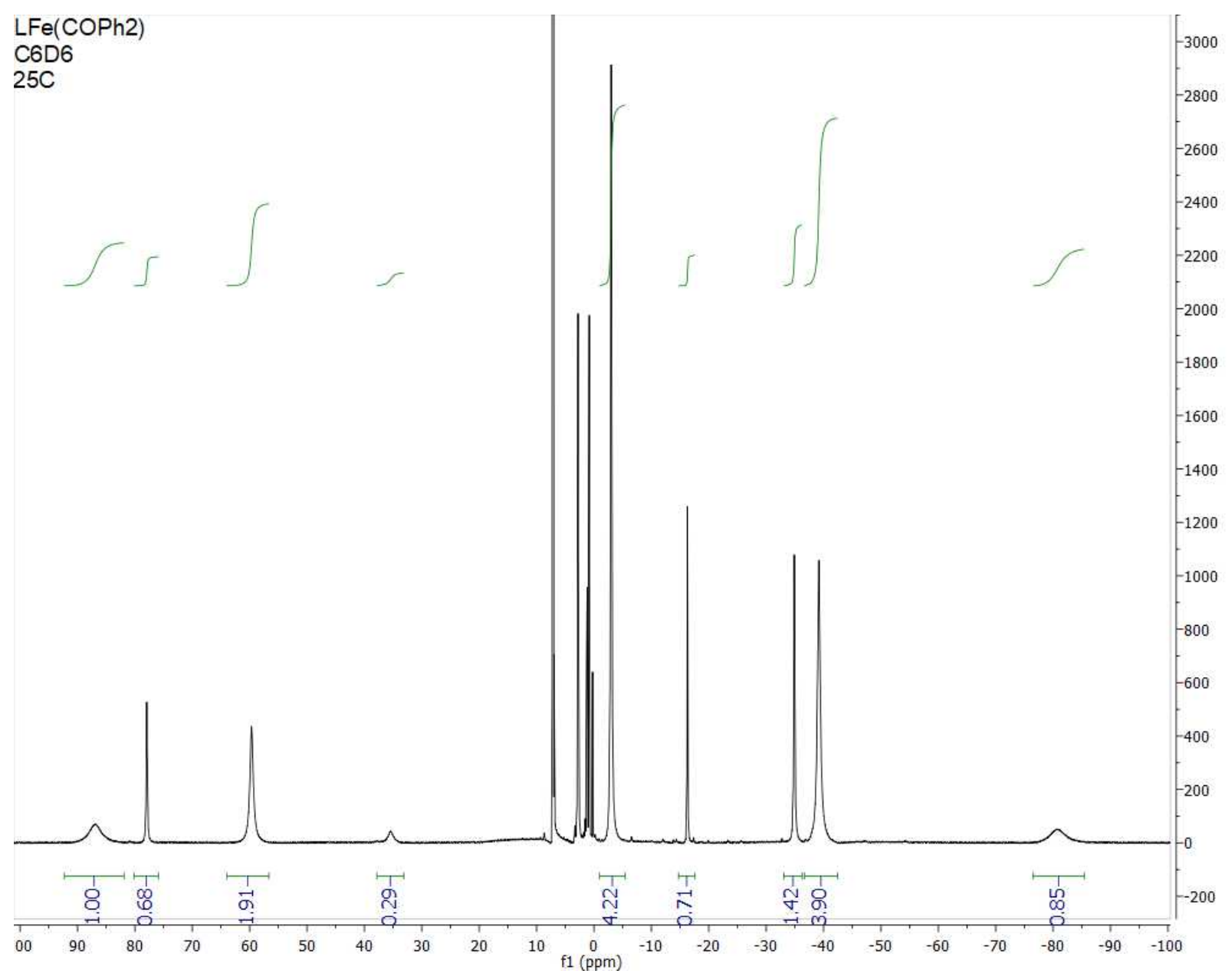

Figure S-6: ${ }^{1} \mathrm{H}$ NMR spectrum of $\mathrm{L}^{\mathrm{Me}} \mathrm{Fe}\left(\mathrm{Ph}_{2} \mathrm{CO}\right)$ (2) in $\mathrm{C}_{6} \mathrm{D}_{6}$. 

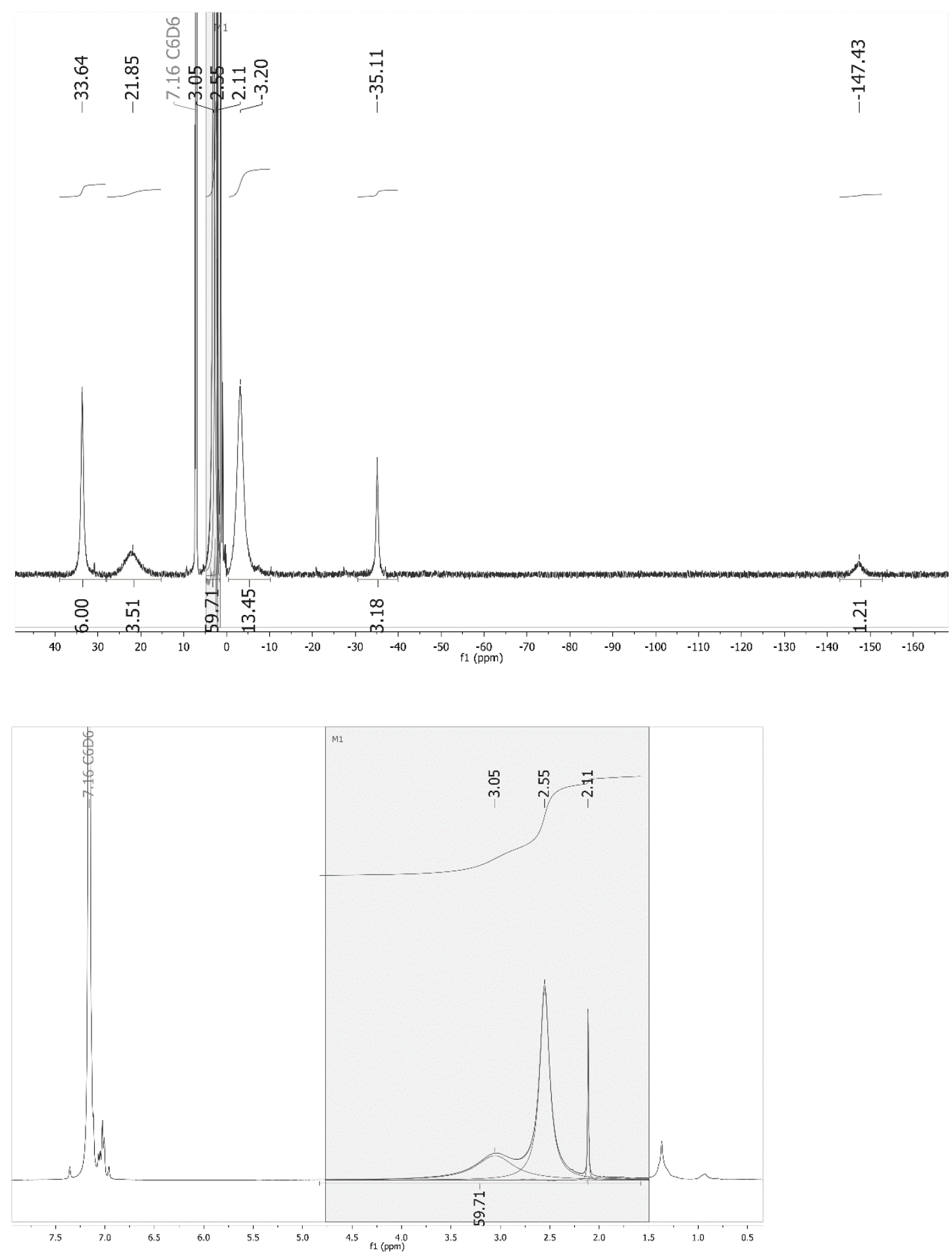

Figure S-7: ${ }^{1} \mathrm{H}$ NMR spectrum of $\left[\mathrm{K}\left(18\right.\right.$-crown-6)] $\left[\mathrm{L}^{\mathrm{Me}} \mathrm{FeCH}_{3}\right]$ (3) in $\mathrm{C}_{6} \mathrm{D}_{6}$ : (top) full spectrum, (bottom) closeup of the region from 0 to $8 \mathrm{ppm}$. 


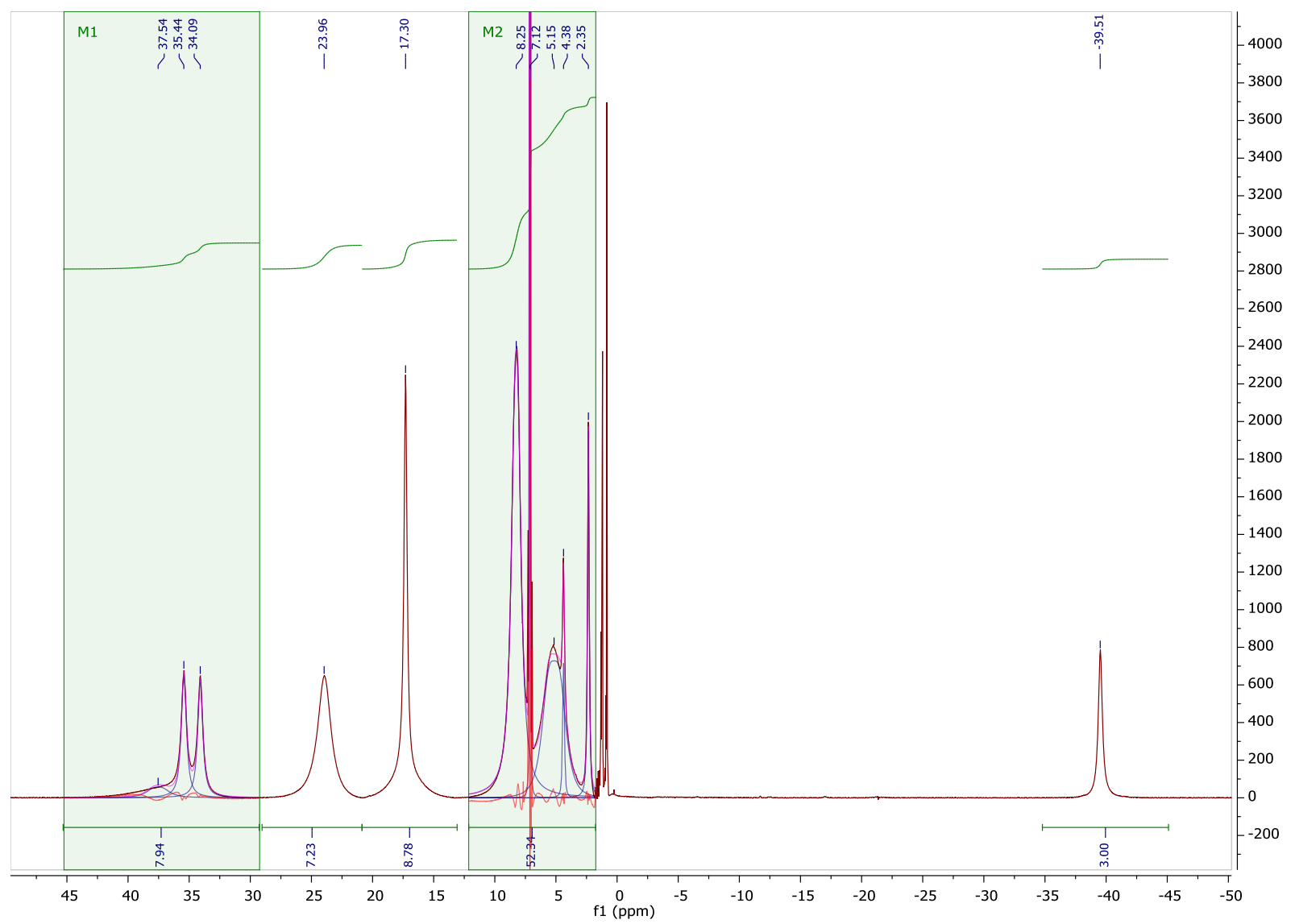

Figure S-8: ${ }^{1} \mathrm{H}$ NMR spectrum of $\mathrm{L}^{\mathrm{Me}} \mathrm{Fe}\left(\mathrm{OCPh}{ }_{2} \mathrm{CHCPh}\right)(\mathbf{6})$ in $\mathrm{C}_{6} \mathrm{D}_{6} .{ }^{1} \mathrm{H} \mathrm{NMR}\left(\mathrm{C}_{6} \mathrm{D}_{6}, 400\right.$ MHz) 37, 35, 34, 24, 17, 8, 5, 4, 2, -40 ppm. There are at least three missing or overlapping signals, which is unsurprising given the broadness of the peaks as is typical for the iron(III) oxidation state. 


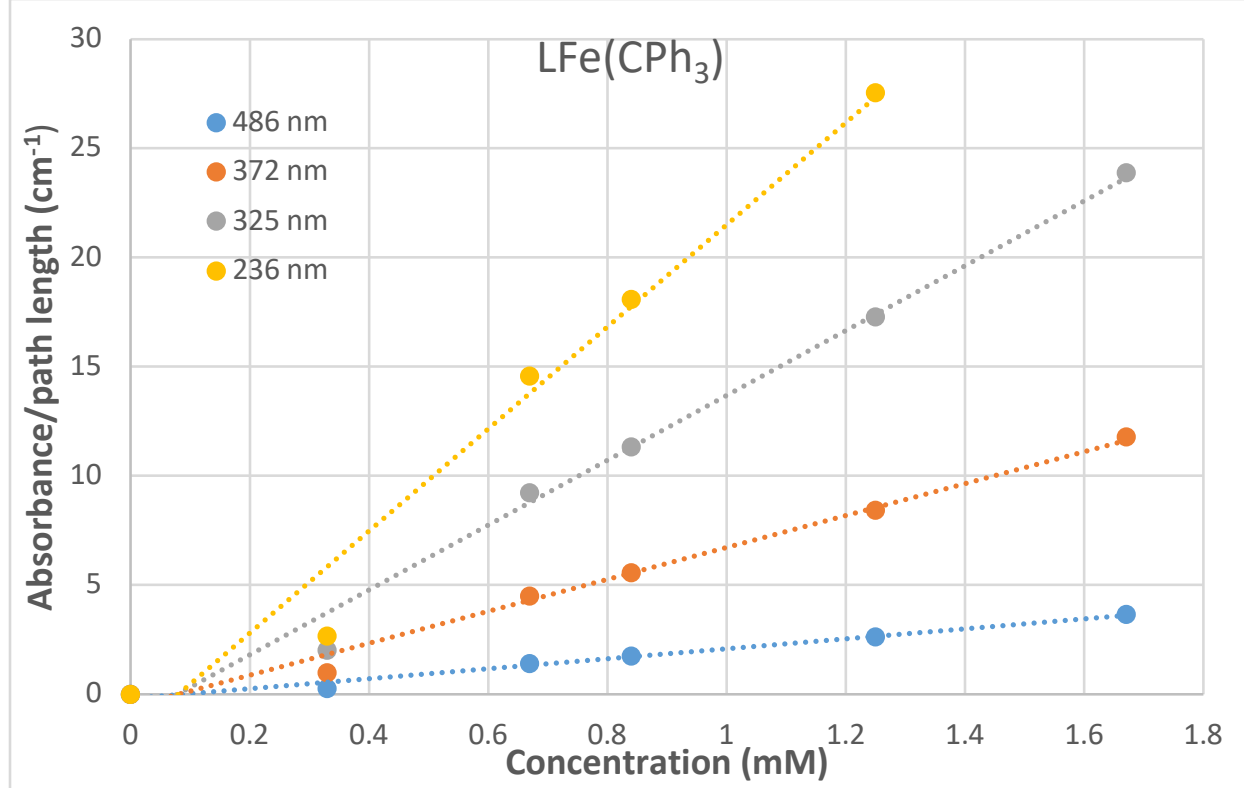

\begin{tabular}{|c|c|c|c|}
\hline & & Value & Error \\
\hline \multirow[t]{3}{*}{ Peak at $486 \mathrm{~nm}$} & $y$-int & -0.4080 & 0.1487 \\
\hline & slope & 2460.1805 & 140.2989 \\
\hline & $\mathrm{R}^{2}$ & 0.9903 & \\
\hline \multirow[t]{3}{*}{ Peak at $372 \mathrm{~nm}$} & $y$-int & -1.1953 & 0.3945 \\
\hline & slope & 7826.3101 & 372.2403 \\
\hline & $\mathrm{R}^{2}$ & 0.9933 & \\
\hline \multirow[t]{3}{*}{ Peak at $325 \mathrm{~nm}$} & $y$-int & -2.3682 & 0.8057 \\
\hline & slope & 15870.1106 & 760.3451 \\
\hline & $\mathrm{R}^{2}$ & 0.9932 & \\
\hline \multirow[t]{3}{*}{ Peak at $236 \mathrm{~nm}$} & $y$-int & -4.89 & 2.07 \\
\hline & slope & 26669.54 & 2460.74 \\
\hline & $\mathrm{R}^{2}$ & 0.9833 & \\
\hline
\end{tabular}

Figure S-9: Beer-Lambert Law plot of selected UV-vis spectral features for $\mathrm{L}^{\mathrm{Me}} \mathrm{Fe}\left(\mathrm{CPh}_{3}\right)(\mathbf{1})$ in hexanes. 


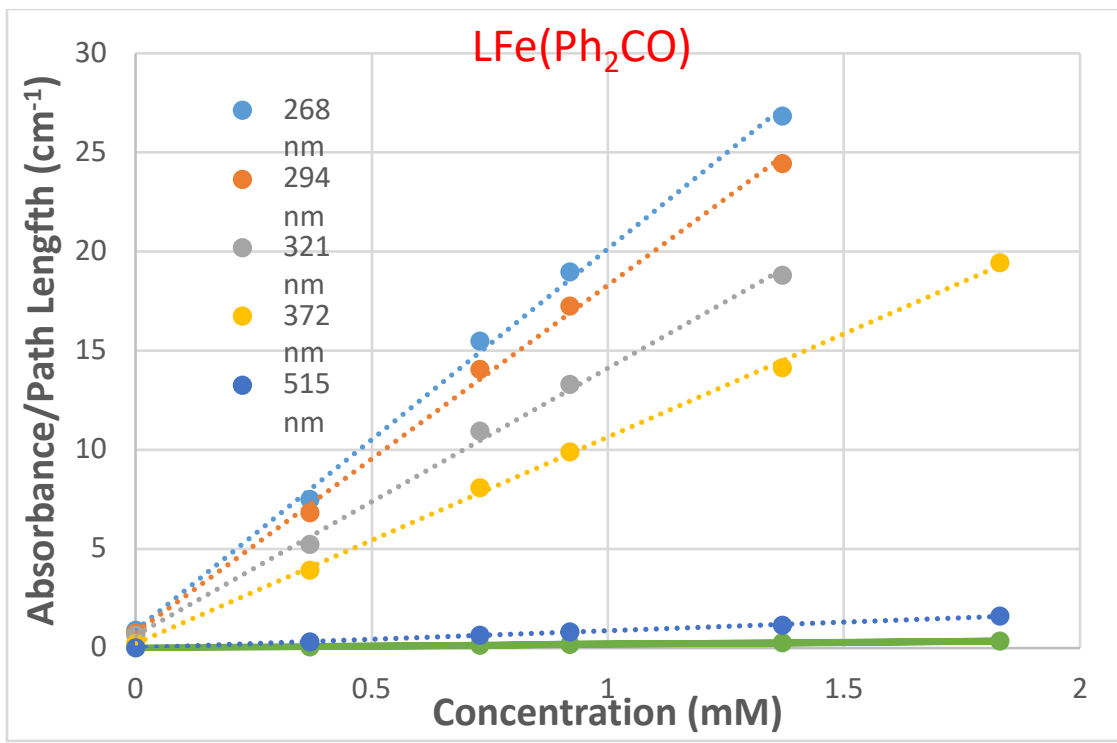

\begin{tabular}{|c|c|c|c|}
\hline & & Value & Error \\
\hline \multirow[t]{3}{*}{ Peak at $817 \mathrm{~nm}$} & $y$-int & 0.004948 & 0.009304 \\
\hline & slope & 188.4428 & 8.012423 \\
\hline & $\mathrm{R}^{2}$ & 0.9946 & \\
\hline \multirow[t]{3}{*}{ Peak at $515 \mathrm{~nm}$} & $y$-int & 0.009976 & 0.031076 \\
\hline & slope & 866.3141 & 26.76175 \\
\hline & $\mathrm{R}^{2}$ & 0.9971 & \\
\hline \multirow[t]{3}{*}{ Peak at $372 \mathrm{~nm}$} & $y$-int & 0.227078 & 0.296108 \\
\hline & slope & 10416.74 & 255.0006 \\
\hline & $\mathrm{R}^{2}$ & 0.9982 & \\
\hline \multirow[t]{3}{*}{ Peak at $321 \mathrm{~nm}$} & $y$-int & 0.640788 & 0.653175 \\
\hline & slope & 13473.72 & 709.1879 \\
\hline & $\mathrm{R}^{2}$ & 0.9945 & \\
\hline \multirow[t]{3}{*}{ Peak at $294 \mathrm{~nm}$} & $y$-int & 0.773263 & 0.729542 \\
\hline & slope & 17529.95 & 792.1041 \\
\hline & $\mathrm{R}^{2}$ & 0.9959 & \\
\hline \multirow[t]{3}{*}{ Peak at $268 \mathrm{~nm}$} & $y$-int & 0.888233 & 0.85134 \\
\hline & slope & 19248.08 & 924.3458 \\
\hline & $\overline{\mathrm{R}^{2}}$ & 0.9954 & \\
\hline
\end{tabular}

Figure S-10: Beer-Lambert Law plot of selected UV-vis spectral features for $\mathrm{L}^{\mathrm{Me}} \mathrm{Fe}\left(\mathrm{Ph}_{2} \mathrm{CO}\right)$ (2) in hexanes solutions. 

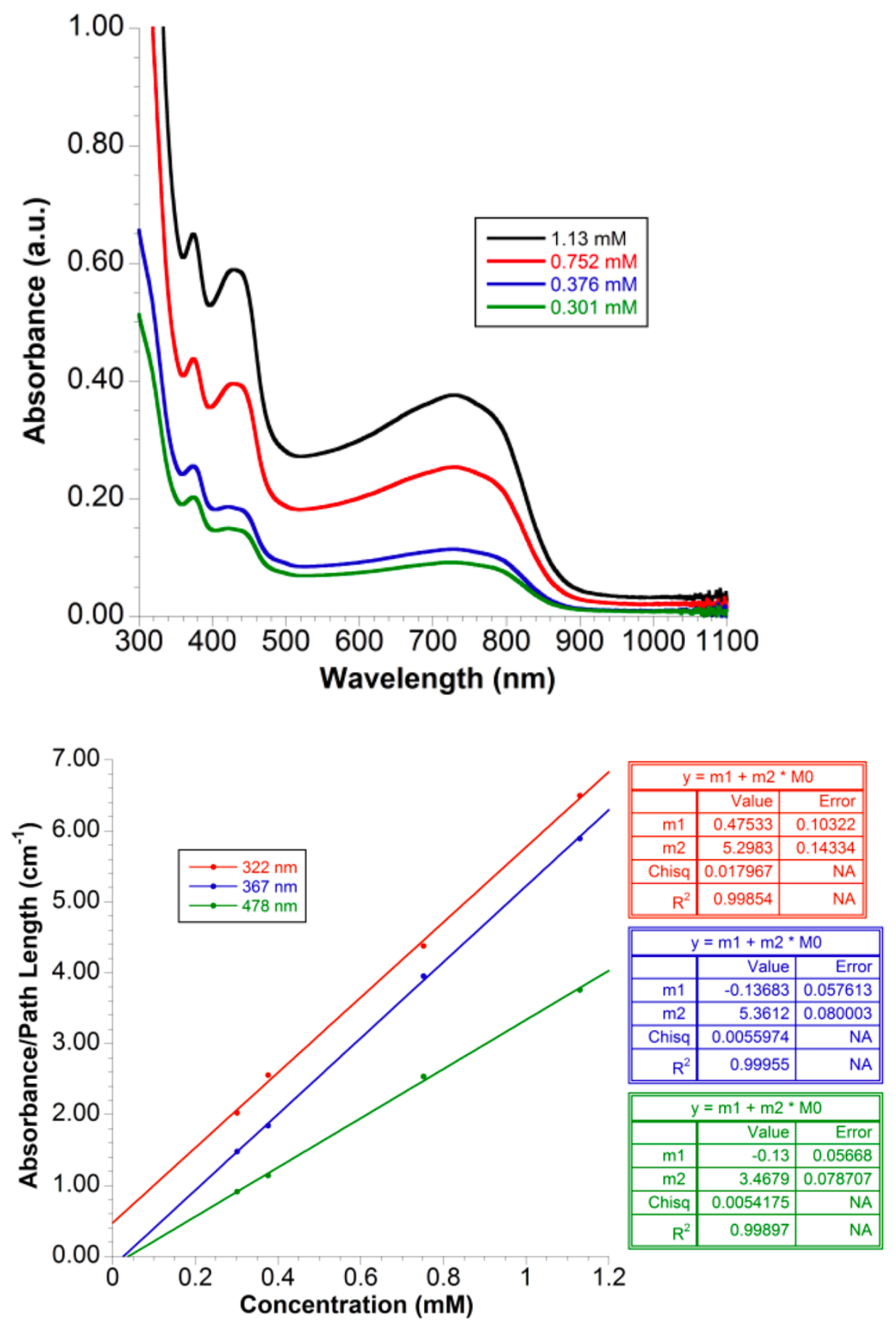

Figure S-11: UV-vis spectra and Beer-Lambert Law plot of $\left[\mathrm{K}\left(18\right.\right.$-crown-6)][L $\left.{ }^{\mathrm{Me}} \mathrm{FeCH} 3\right]$ (3). 


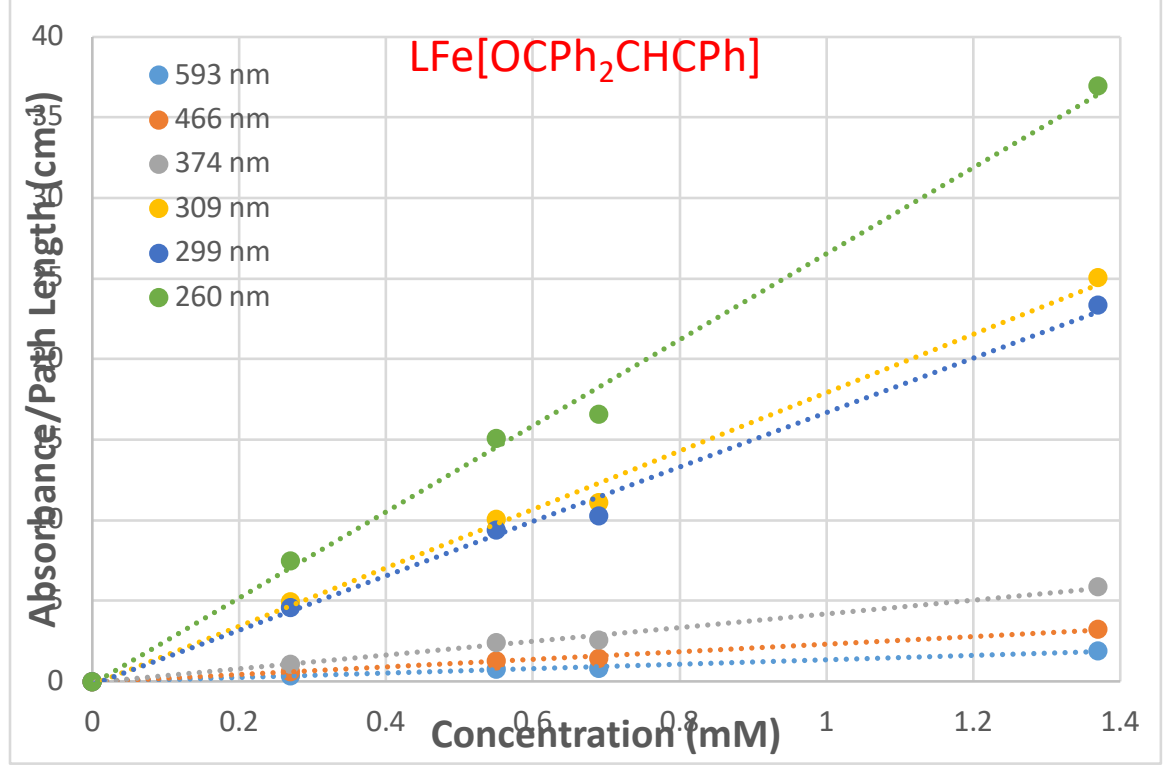

\begin{tabular}{|c|c|c|c|}
\hline & & Value & Error \\
\hline \multirow[t]{3}{*}{ Peak at $593 \mathrm{~nm}$} & $y$-int & -0.0310 & 0.04551 \\
\hline & slope & 1.3712 & 0.06161 \\
\hline & $\mathrm{R}^{2}$ & 0.9940 & \\
\hline \multirow[t]{3}{*}{ Peak at $466 \mathrm{~nm}$} & $y$-int & -0.0435 & 0.07676 \\
\hline & slope & 2.3544 & 0.10392 \\
\hline & $\mathrm{R}^{2}$ & 0.9942 & \\
\hline \multirow[t]{3}{*}{ Peak at $374 \mathrm{~nm}$} & $y$-int & -0.0668 & 0.14701 \\
\hline & slope & 4.25707 & 0.19901 \\
\hline & $\mathrm{R}^{2}$ & 0.9935 & \\
\hline \multirow[t]{3}{*}{ Peak at $309 \mathrm{~nm}$} & $y$-int & -0.2043 & 0.56737 \\
\hline & slope & 18.1270 & 0.76806 \\
\hline & $\mathrm{R}^{2}$ & 0.9946 & \\
\hline \multirow[t]{3}{*}{ Peak at $299 \mathrm{~nm}$} & $y$-int & -0.2057 & 0.53804 \\
\hline & slope & 16.8942 & 0.72836 \\
\hline & $\mathrm{R}^{2}$ & 0.9945 & \\
\hline \multirow[t]{3}{*}{ Peak at $260 \mathrm{~nm}$} & $y$-int & -0.1679 & 0.7940 \\
\hline & slope & 26.7166 & 1.0748 \\
\hline & $\mathrm{R}^{2}$ & 0.9952 & \\
\hline
\end{tabular}

Figure S-12: Beer-Lambert Law plot of selected UV-vis spectral features for $\mathrm{L}^{\mathrm{Me}} \mathrm{Fe}\left(\mathrm{OCPh}{ }_{2} \mathrm{CHCPh}\right)(6)$ in hexanes. 


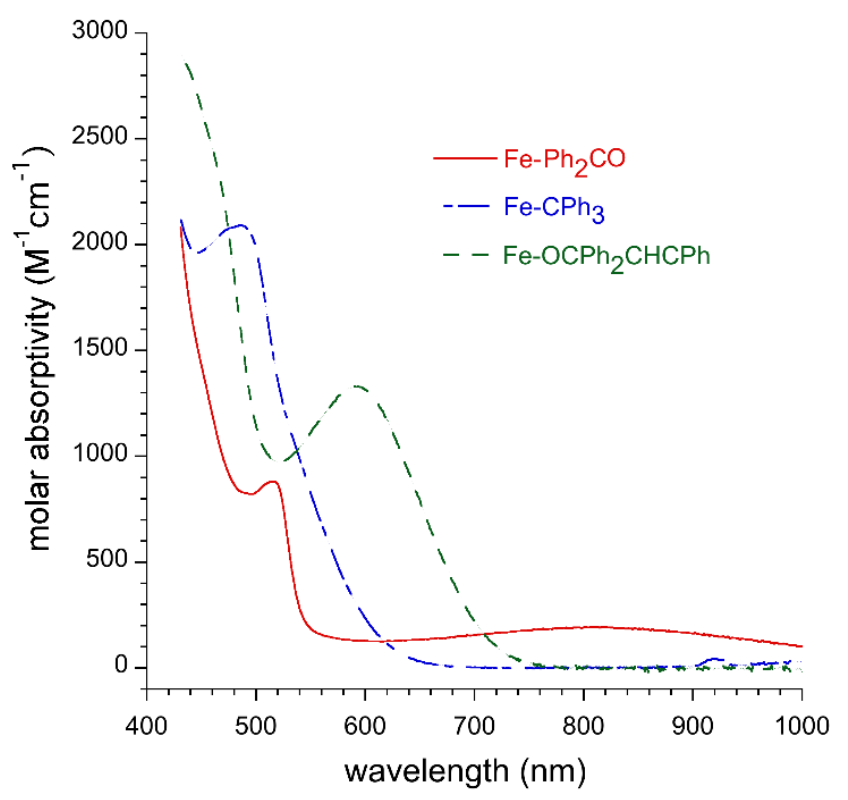

Figure S-13. Overlaid UV-vis spectra of $\mathbf{1}$ (blue), $\mathbf{2}$ (red), and $\mathbf{6}$ (green). 


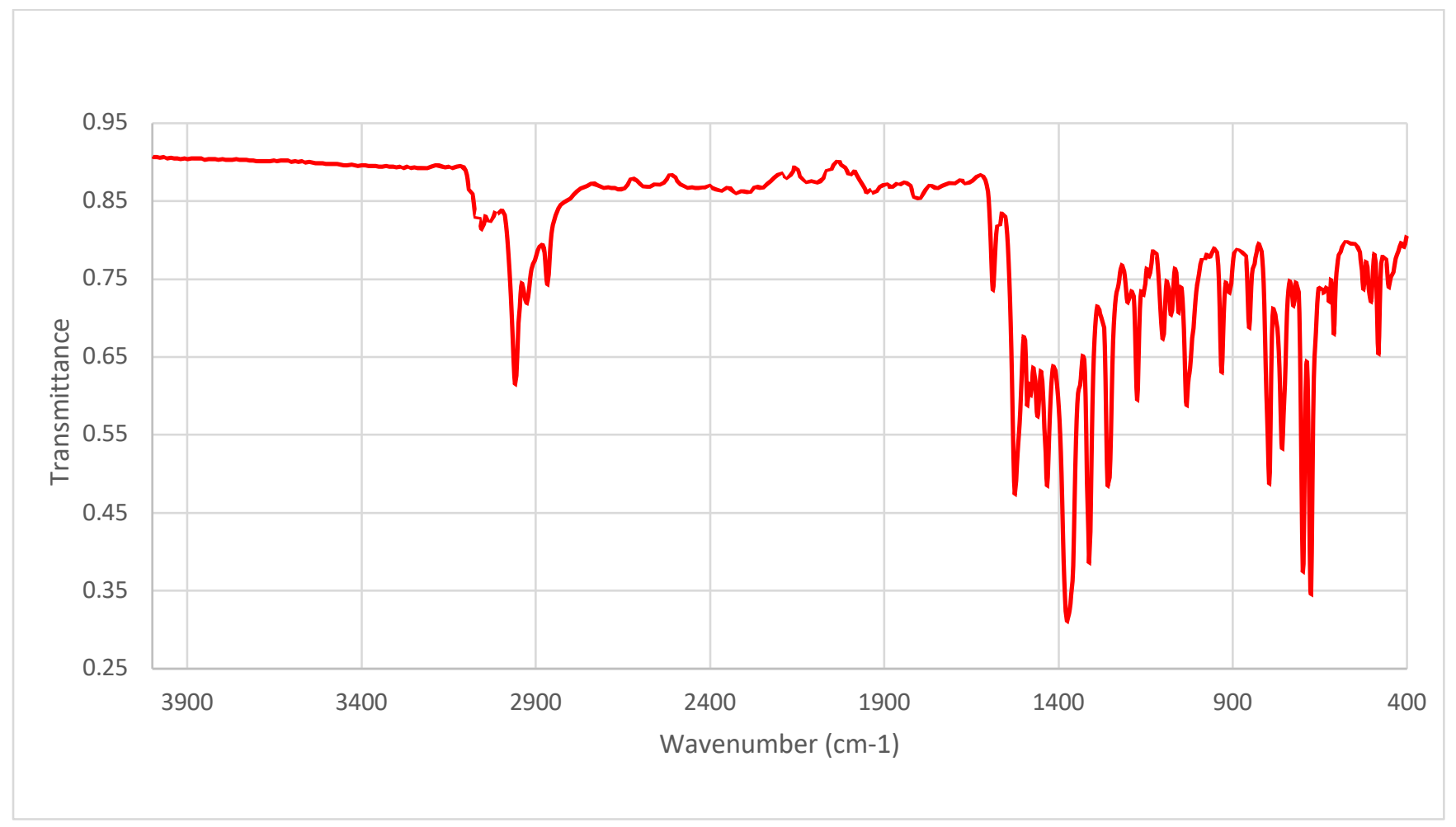

Figure S-14: FTIR spectrum of solid $\mathrm{L}^{\mathrm{Me}} \mathrm{Fe}\left(\mathrm{CPh}_{3}\right)$ (1). 


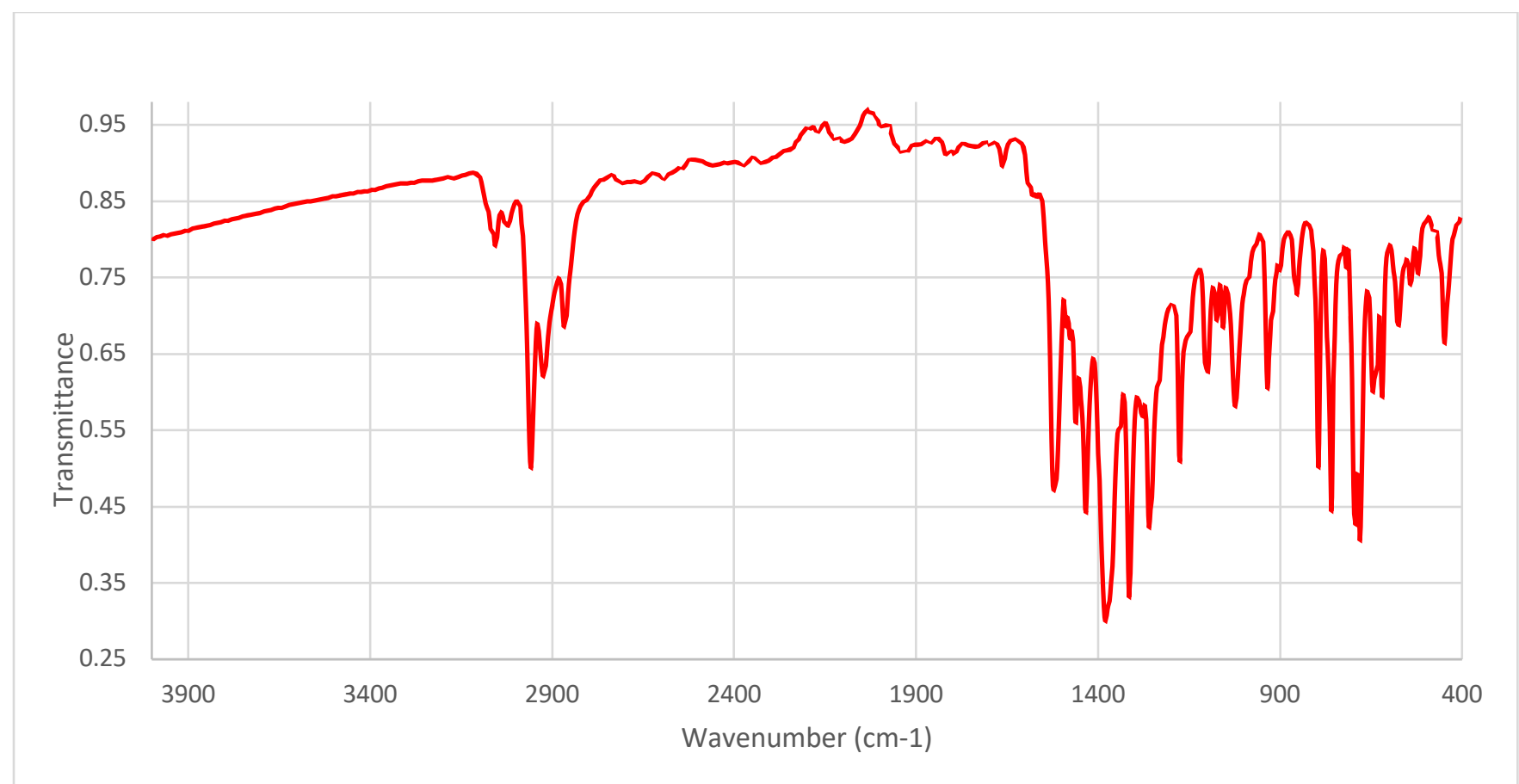

Figure S-15: FTIR spectrum of solid $\mathrm{L}^{\mathrm{Me}} \mathrm{Fe}\left(\mathrm{Ph}_{2} \mathrm{CO}\right)$ (2). 


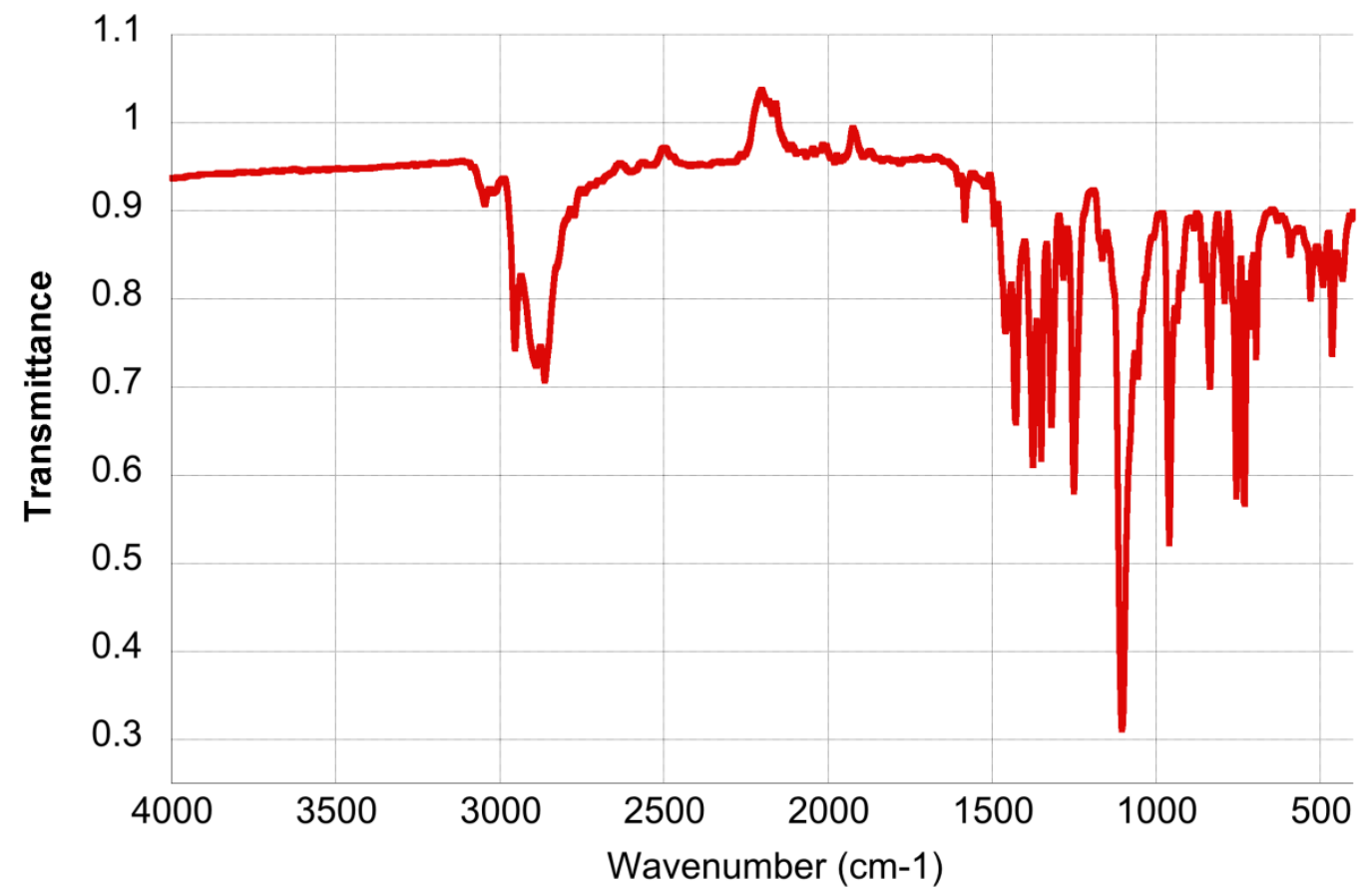

Figure S-16: FTIR spectrum of [K(18-crown-6)][L $\left.\mathrm{L}^{\mathrm{Me}} \mathrm{FeCH} 3\right]$ (3). 


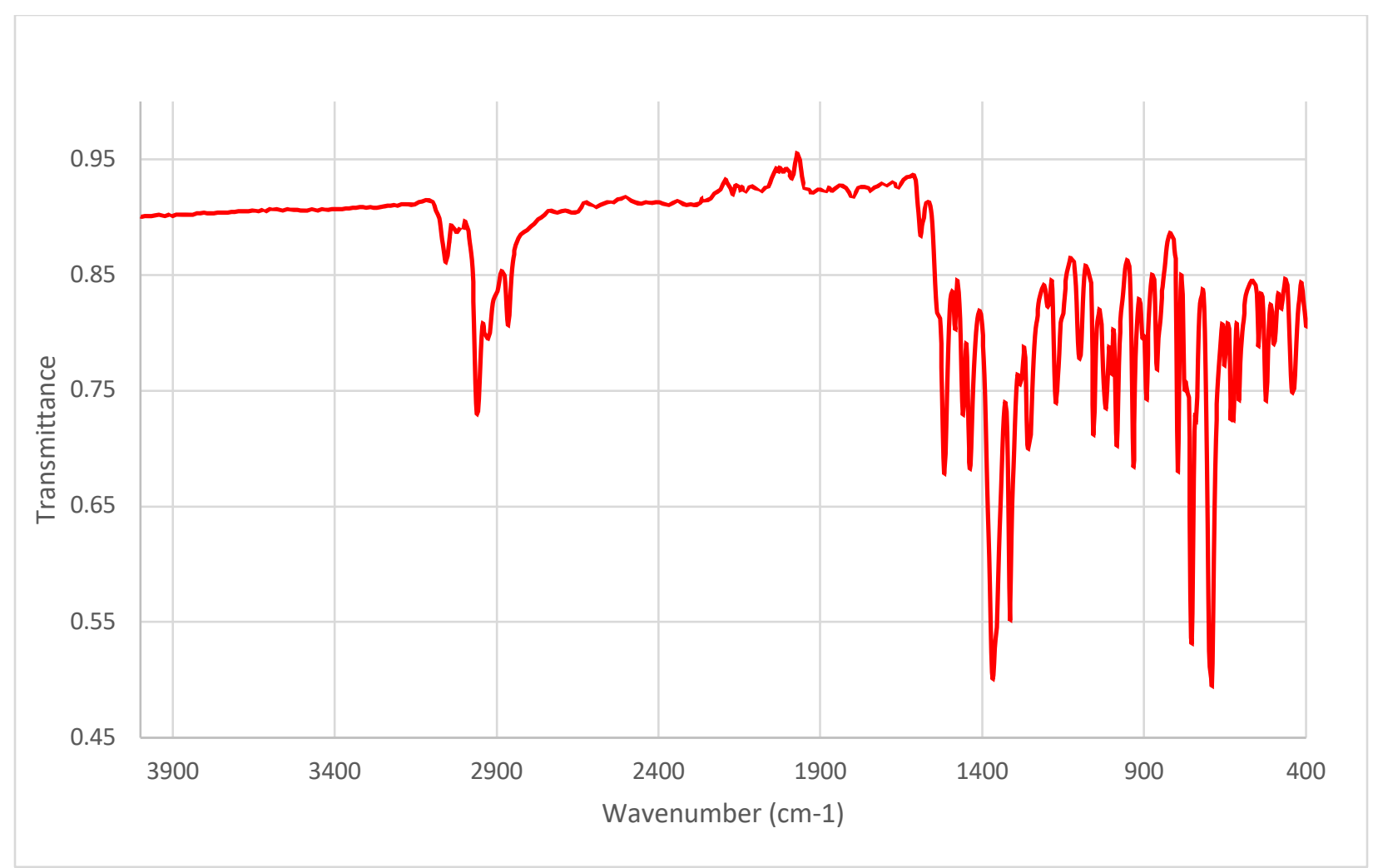

Figure S-17: FTIR spectrum of $\mathrm{L}^{\mathrm{Me}} \mathrm{Fe}\left(\mathrm{OCPh}_{2} \mathrm{CHCPh}\right)$ (6). 
X-Ray Absorption Data Collection. Fe K-edge XAS spectra were collected on the 16 pole, $2 \mathrm{~T}$ wiggler beamline 9-3 at the Stanford Synchrotron Radiation Lightsource (SSRL) under ring conditions of $3 \mathrm{GeV}$ and $500 \mathrm{~mA}$. Samples were diluted in BN, pressed into $1 \mathrm{~mm}$ aluminum spacers and sealed with $37 \mu \mathrm{m}$ Kapton tape. Samples were maintained at $10 \mathrm{~K}$ in a liquid $\mathrm{He}$ cryostat during data collection. A Si(220) double-crystal monochromator was used for energy selection and a Rh-coated mirror (set to an energy cutoff of $13 \mathrm{keV}$ ) was used for harmonic rejection. Internal energy calibration was performed by assigning the first inflection point of an Fe foil spectrum to $7112.6 \mathrm{eV}$. ${ }^{1}$ Spectra were collected in fluorescence mode with a Lytle detector, attenuating elastic scatter into the detector using a Soller slit with an upstream $\mathrm{Mn}$ filter. The raw data were averaged and energy shifted using EXAFSPAK. ${ }^{1}$ Data were calibrated using an internal Fe foil standard. Spectra were shifted such that the Fe foil rising edge inflection point matched the value of $7112.6 \mathrm{eV}$. The averaged data file was then normalized in Igor by applying a linear normalization to the pre-edge and a quad normalization to the post-edge to produce the final spectra.

Computational Details. All electronic structure and spectroscopic calculations were performed using the ORCA 4.04 computational chemistry package. ${ }^{2}$ Geometry optimizations were performed using the BP86 functional, ${ }^{3}$ the zeroth-order regular approximation for relativistic effects (ZORA) ${ }^{4}$ as implemented by van Wüllen, ${ }^{5}$ and the CP(PPP) basis set. ${ }^{6}$ Optimized crystal structure coordinates were used for TDDFT calculations ${ }^{7}$ of Fe K-edge XAS utilizing the B3LYP ${ }^{9}$ functional. Calculated excitation energies were plotted against experimental energies for the B3LYP functional. ${ }^{8}$ This correlation (Figure S-18) was used to shift the calculated energy of the spectra and produce calculated spectra that correlate well to experimental spectra. 


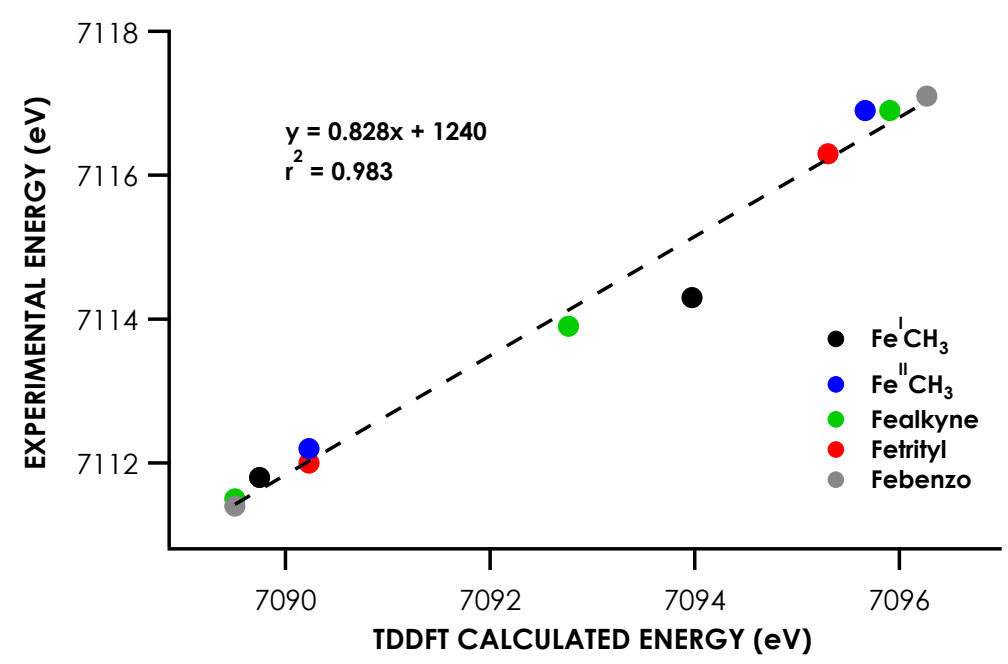

Figure S-18: Correlation curve of experimentally collected pre-edge energy to B3LYP calculated energy for compounds $\mathbf{1 - 5}$ used to shift calculated spectra.

Multireference character in the ground states of $\mathbf{2}$ and $\mathbf{5}$ was evaluated using SORCI calculations. SORCI was performed on a complete active space (CAS) for models of $\mathbf{2}$ and $\mathbf{5}$ comprising 15 electrons and 11 orbitals [CAS(15,11)]. Sufficiency of the active space was evaluated by ensuring that it captured ca. $90 \%$ of chosen state references without requiring holes or particles outside the active orbitals. The ZORA-def2-TZVPP(-f) basis set $^{9}$ was used on Fe, and ZORAdef2-SVP was used on all other atoms. The ZORA relativistic correction ${ }^{10}$ was used in all SORCI calculations. As described elsewhere, ${ }^{11}$ individual selection was used to ease the computational burden. The size of the first-order interacting space was reduced with a threshold: $T_{\text {sel }}=10^{-} \mathrm{E}_{\mathrm{h}}$. A further approximation involved reducing the reference space through another selection: all initial references that contributed less than a second threshold $\left(T_{p r e}=10^{-s}\right)$ to the zeroth-order states were rejected from the reference space. Starting orbitals were taken from unrestricted Kohn-Sham orbitals generated via B3LYP calculations using the aforementioned basis sets that were subsequently transformed to quasi-restricted orbitals (QROs). ${ }^{12}$ These orbitals were then used in a CASSCF calculation, whereupon the resulting orbitals were used in the SORCI procedure. 


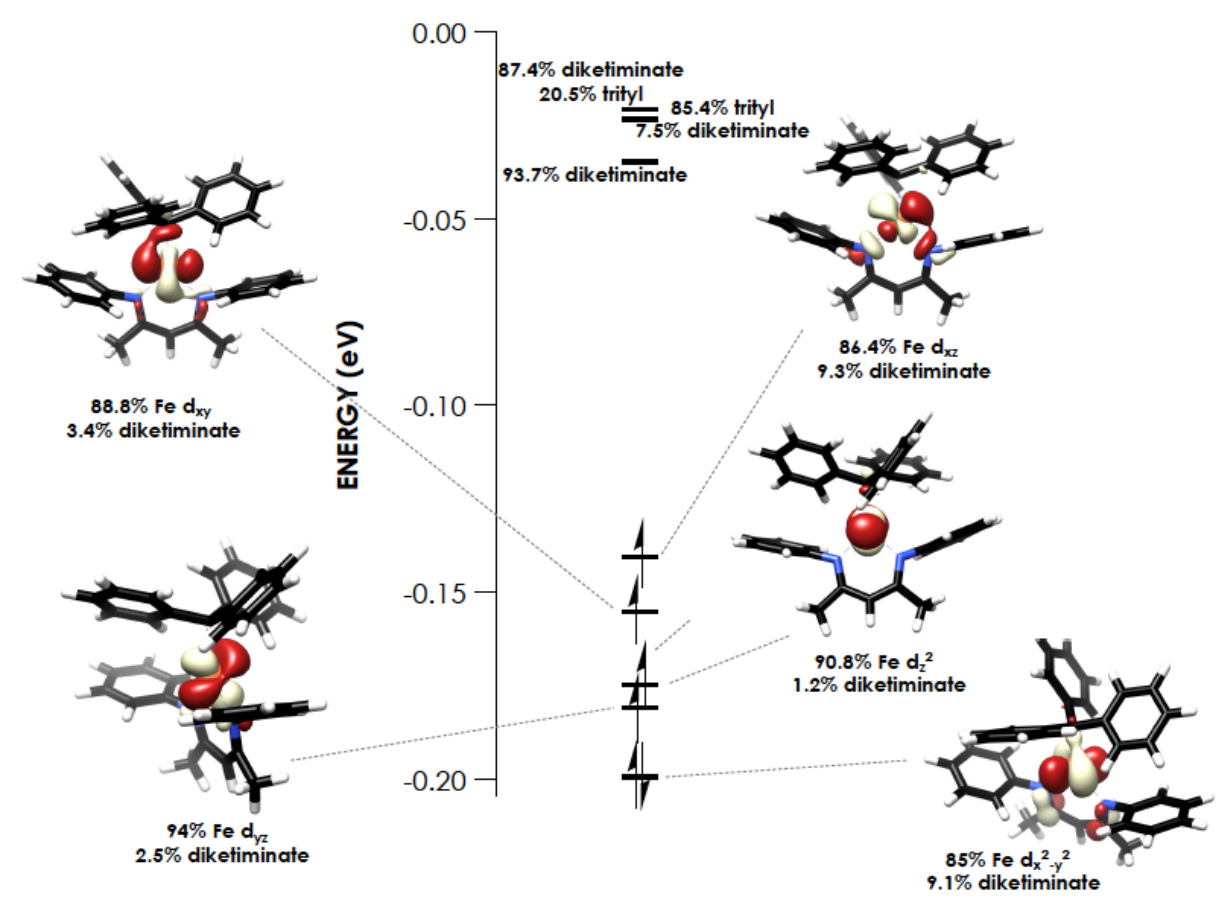

Figure S-19. (left) Overlay of experimental and TDDFT calculated spectra of 1 showing acceptor molecular orbitals for pre-edge transitions. (right) Molecular orbital diagram for 1 generated with QROs calculated with the B3LYP functional. Orbitals are plotted at an isovalue of $0.03 \mathrm{au}$. 


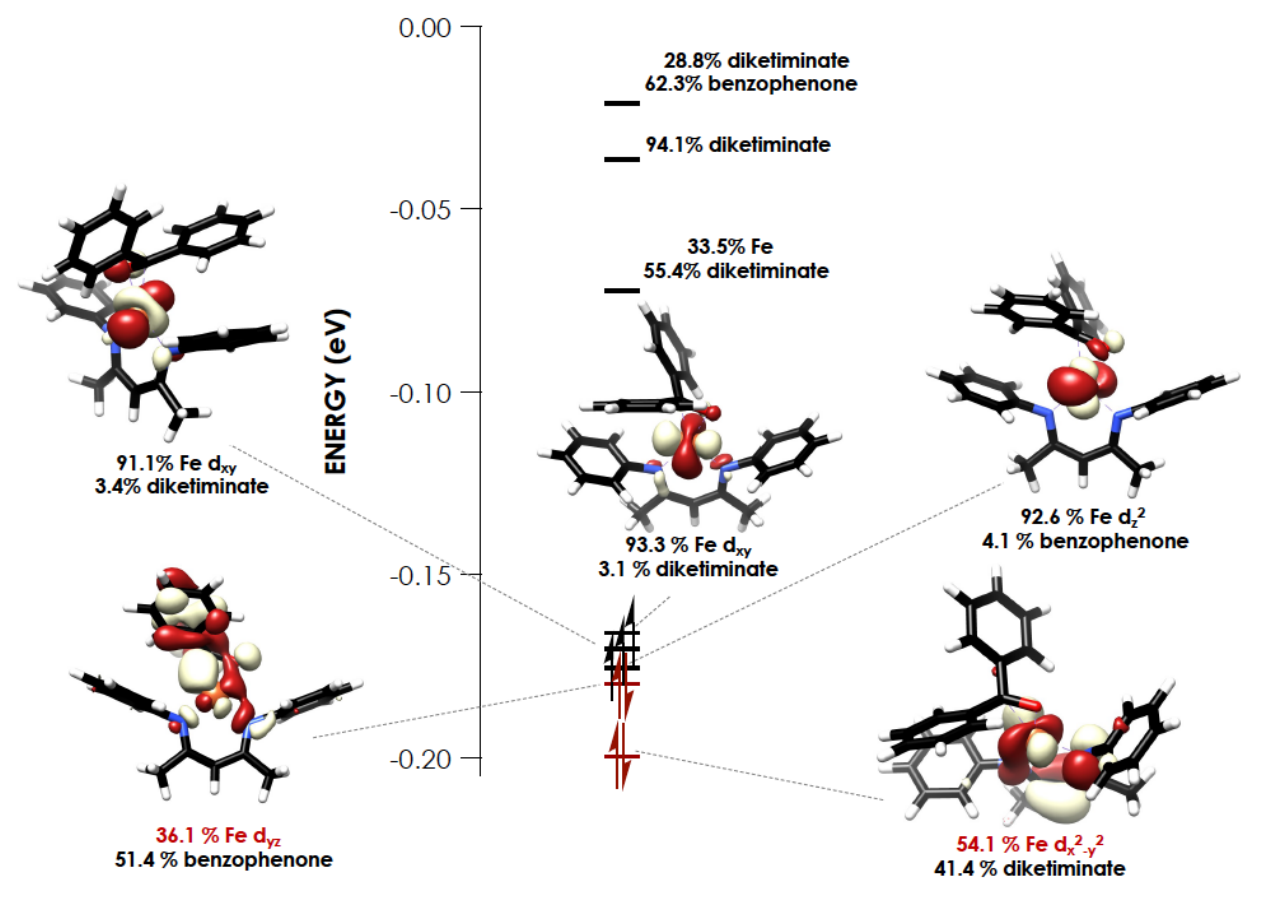

Figure S-20. Molecular orbital diagram for 2 generated with QROs calculated with the B3LYP functional. Orbitals with low Fe percentage are shown in red. Orbitals are plotted at an isovalue of 0.03 au. 


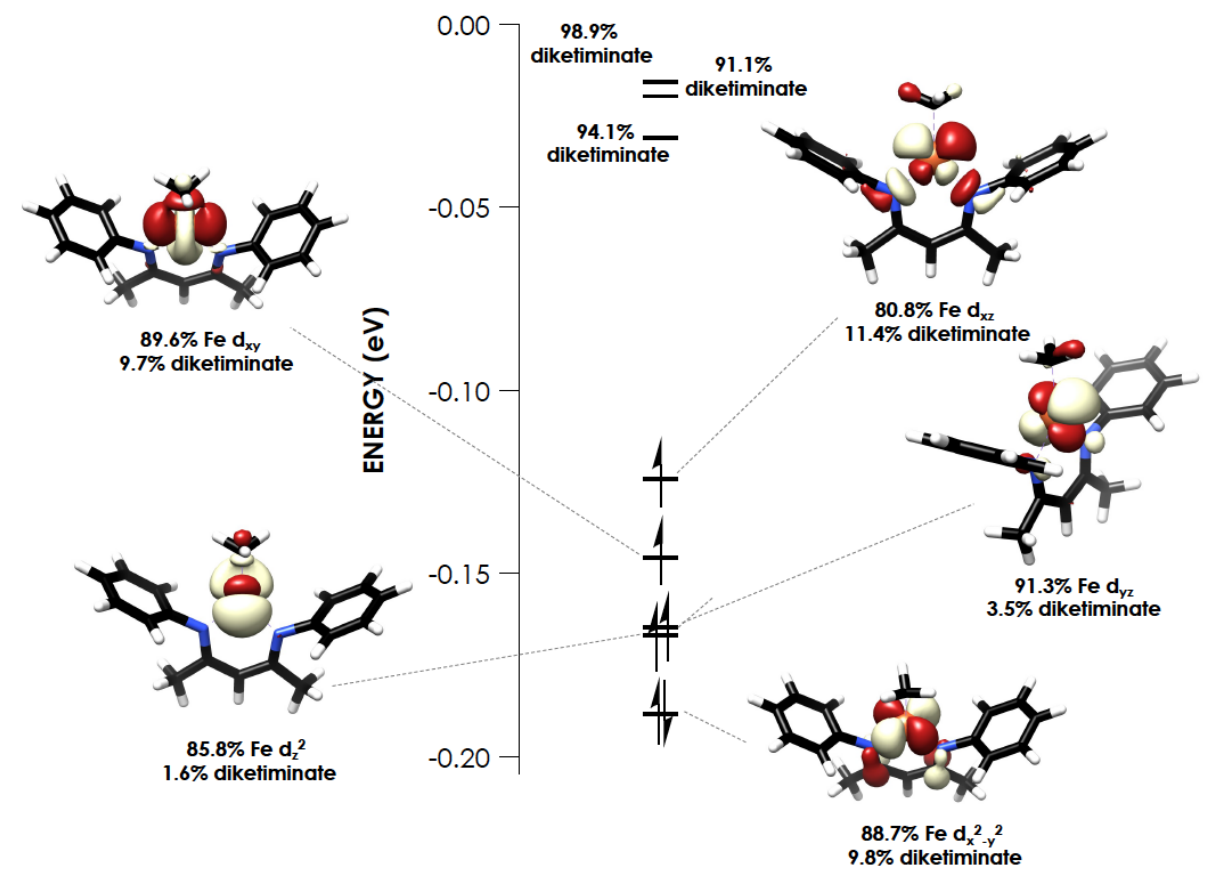

Figure S-21. Molecular orbital diagram for 3 generated with QROs calculated with the B3LYP functional. Orbitals are plotted at an isovalue of 0.03 au. 


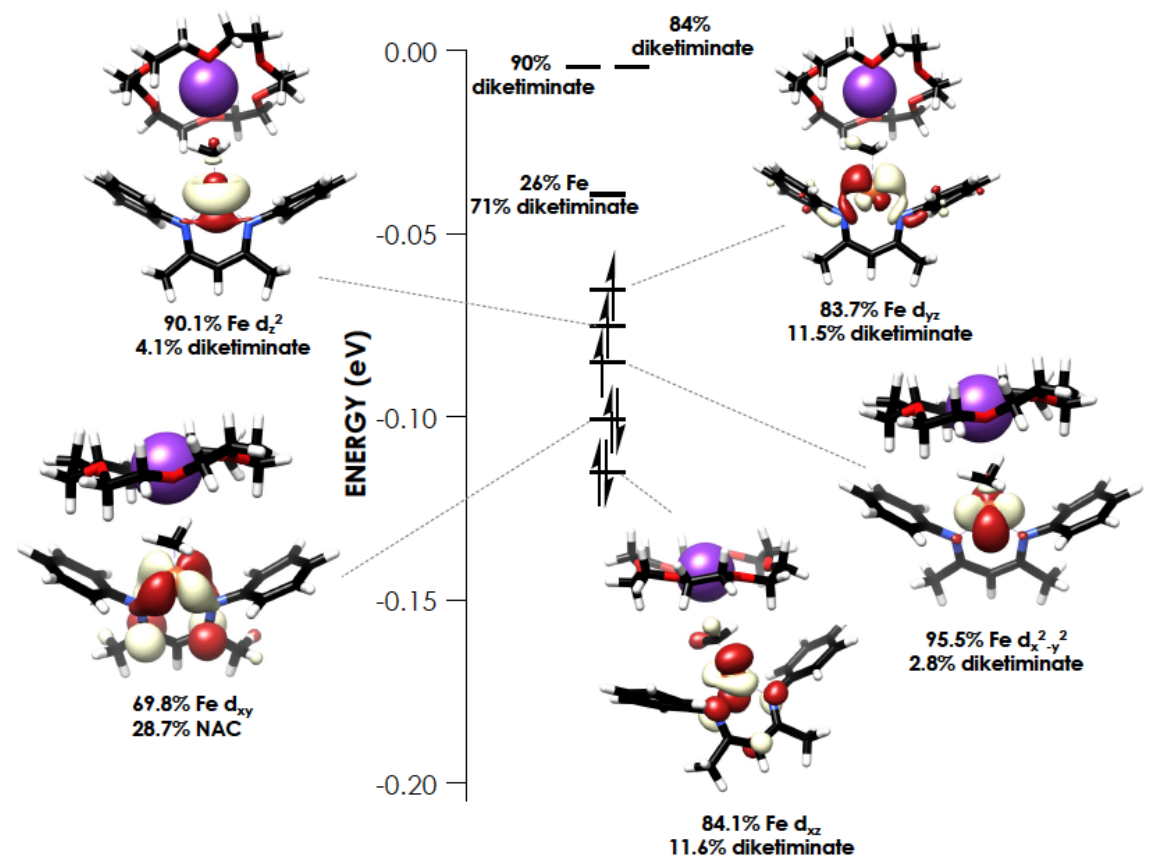

Figure S-22. Molecular orbital diagram for 4 generated with QROs calculated with the B3LYP functional. Orbitals are plotted at an isovalue of 0.03 au. 


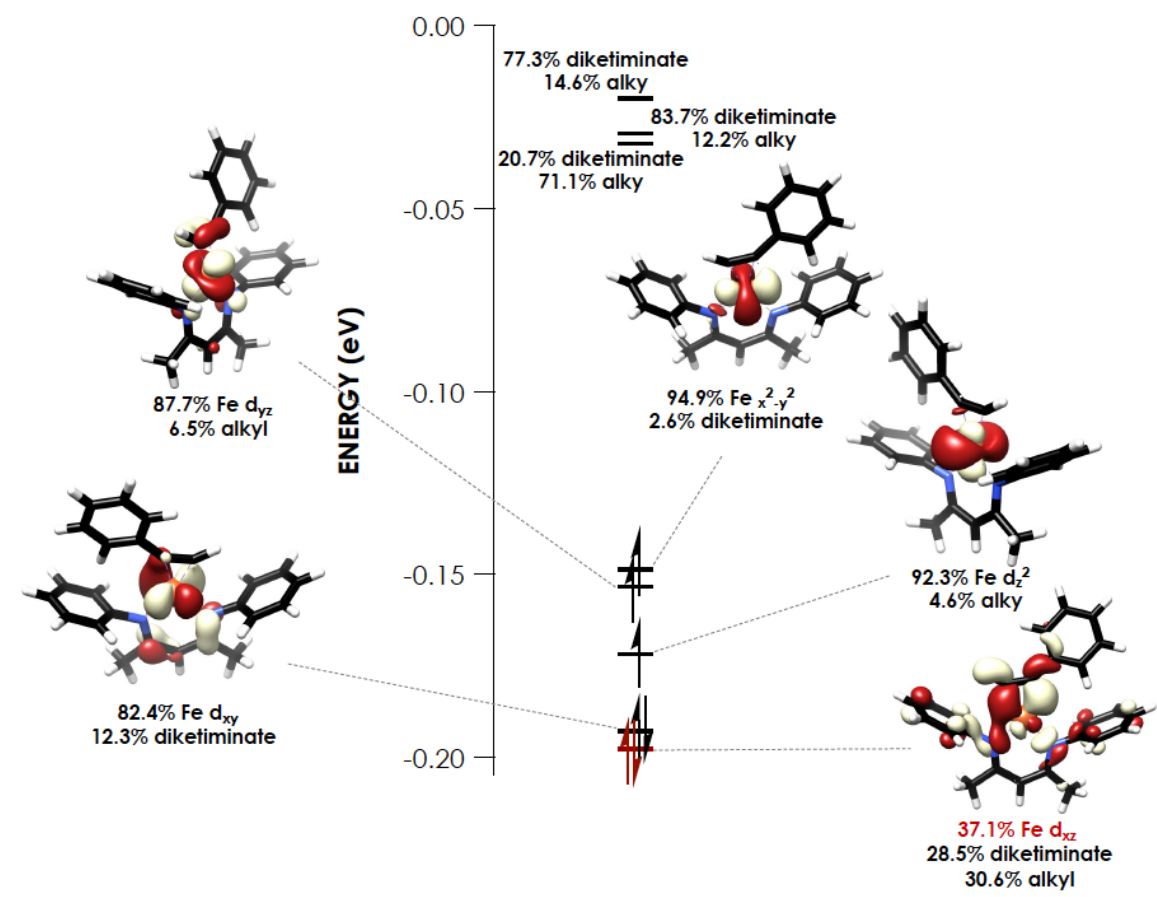

Figure S-23. Molecular orbital diagram for 5 generated with QROs calculated with the B3LYP functional. Orbitals are plotted at an isovalue of $0.03 \mathrm{au}$. 


\section{Example input file: geometry optimizations}

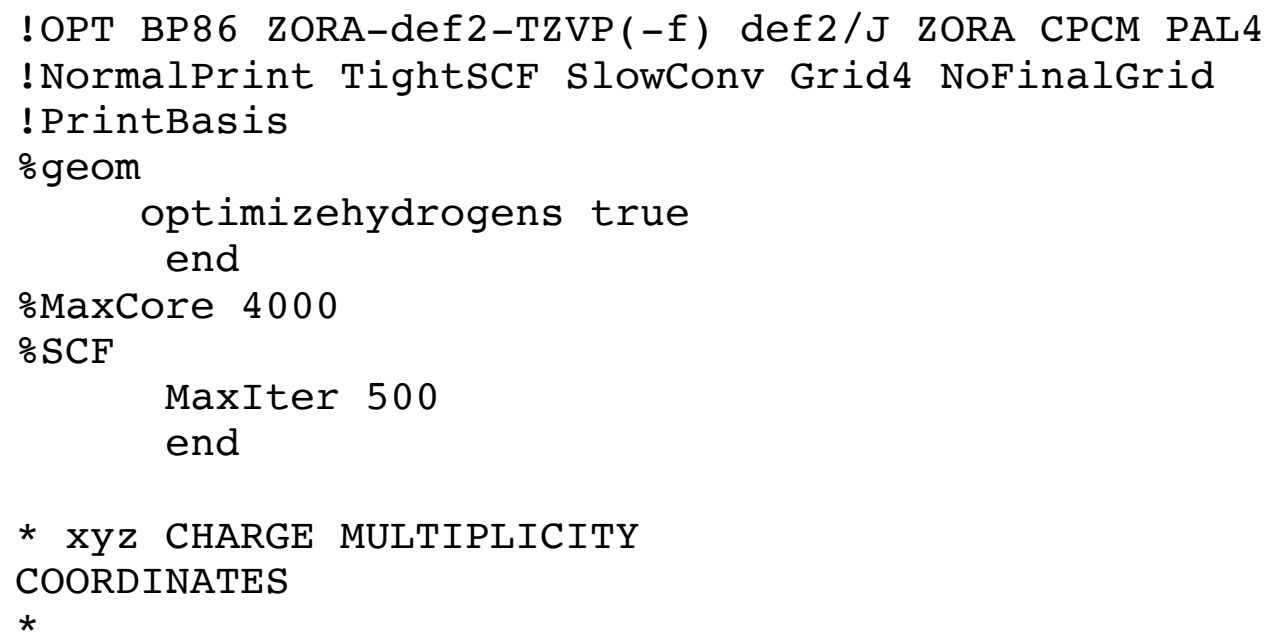

Example input file: TD-DFT for K-edge spectra

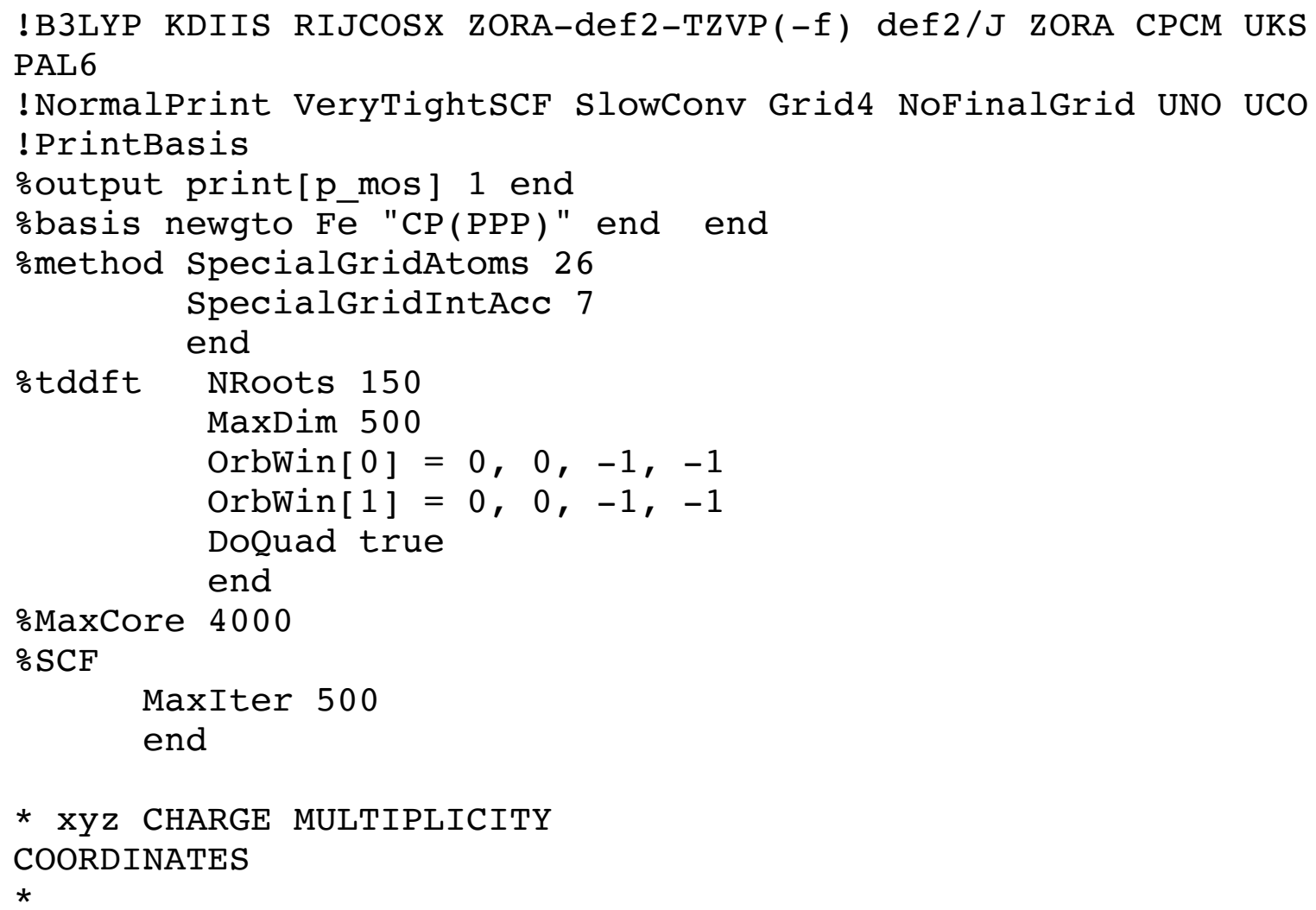




\section{Example input file: SORCI}

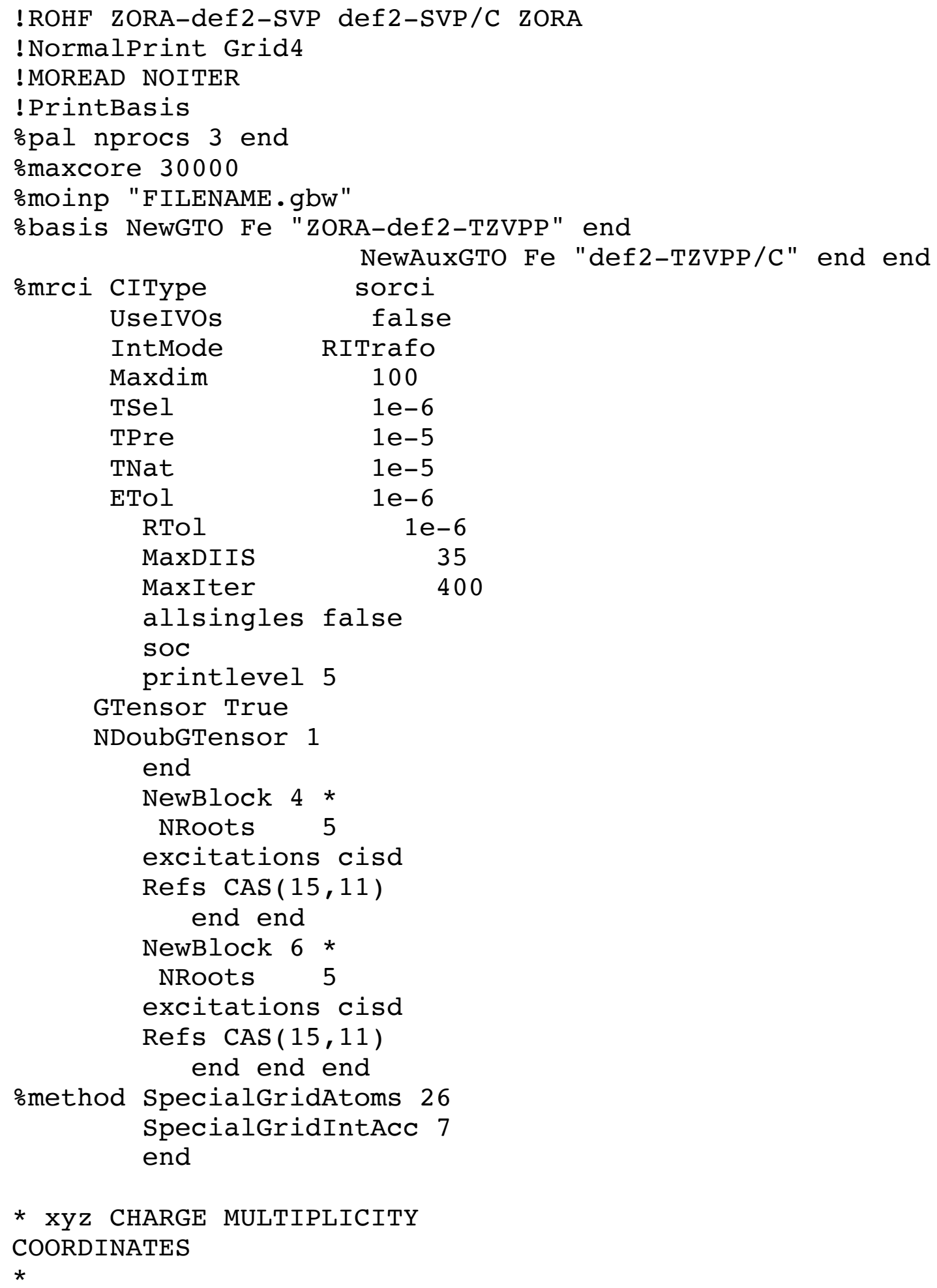




\section{Example input file: CASSCF}

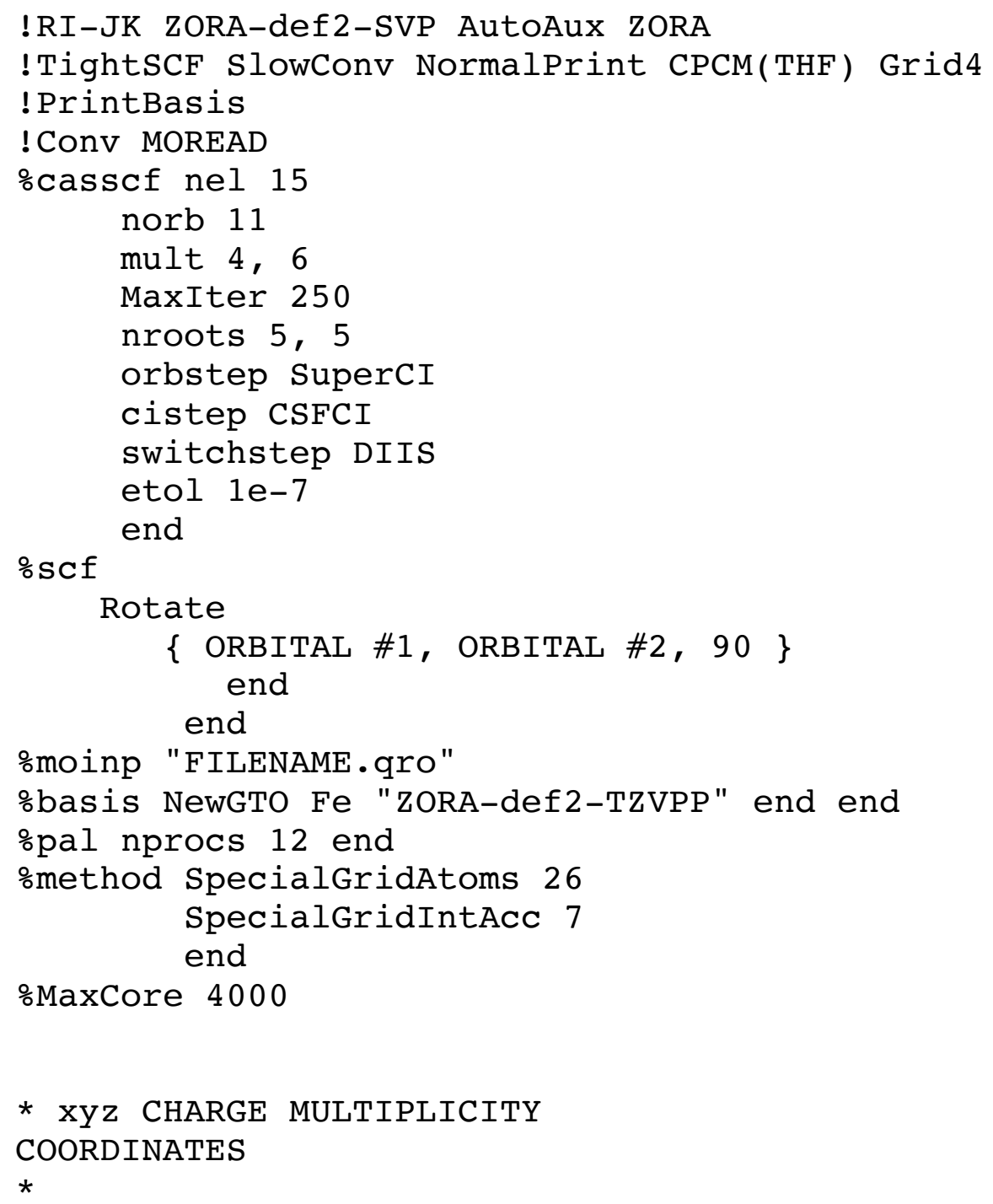




\section{Optimized, Truncated Structure for 1}

$$
\begin{aligned}
& \begin{array}{llll}
\text { Fe } & 0.000000 & 0.000000 & 0.000000
\end{array} \\
& \begin{array}{llll}
\mathrm{N} & -1.212610 & -0.734180 & -1.428340
\end{array} \\
& \text { N } \quad \begin{array}{lll}
1.307280 & 0.790110 & -1.303680
\end{array} \\
& \begin{array}{llll}
\text { C } & 0.402220 & -0.386710 & -3.200820
\end{array} \\
& \begin{array}{llll}
\mathrm{H} & 0.576390 & -0.586360 & -4.256580
\end{array} \\
& \begin{array}{llll}
\text { C } & 3.496010 & -2.104410 & 3.579800
\end{array} \\
& \mathrm{H} \quad 4.341510 \quad-2.671230 \quad 3.974690 \\
& \begin{array}{llll}
\text { C } & 0.961720 & 2.343010 & 2.275240
\end{array} \\
& \mathrm{H} \quad 1.882490 \quad 1.952840 \quad 1.841150 \\
& \begin{array}{llll}
\text { C } & -1.261270 & 2.040580 & 3.078810
\end{array} \\
& \mathrm{H} \quad-2.121360 \quad 1.410740 \quad 3.303630 \\
& \begin{array}{llll}
\text { C } & 2.015820 & -0.213300 & 3.699240
\end{array} \\
& \begin{array}{llll}
\mathrm{H} & 1.703480 & 0.694770 & 4.216750
\end{array} \\
& \begin{array}{llll}
\text { C } & 1.559540 & 3.193750 & -0.983620
\end{array} \\
& \begin{array}{llll}
\text { C } & -1.168720 & -0.843930 & 2.596430
\end{array} \\
& \begin{array}{llll}
\text { C } & 2.117610 & 1.906690 & -0.878260
\end{array} \\
& \begin{array}{llll}
\text { C } & 3.402180 & 1.711760 & -0.347800
\end{array} \\
& \begin{array}{llll}
\text { C } & -2.576740 & -1.114220 & -1.146850
\end{array} \\
& \begin{array}{llll}
\text { C } & -3.546860 & -0.094450 & -1.029310
\end{array} \\
& \begin{array}{llll}
\text { C } & -2.914790 & -2.459720 & -0.985630
\end{array} \\
& \begin{array}{llll}
\text { C } & 1.741620 & -1.820950 & 1.963780
\end{array} \\
& \begin{array}{llll}
\mathrm{H} & 1.197160 & -2.208190 & 1.094860
\end{array} \\
& \begin{array}{llll}
\text { C } & -0.114400 & 1.471440 & 2.501660
\end{array} \\
& \begin{array}{llll}
\text { C } & -0.825060 & -0.844440 & -2.708400
\end{array} \\
& \begin{array}{llll}
\text { C } & 1.335040 & 0.461560 & -2.595150
\end{array} \\
& \begin{array}{llll}
\text { C } & 0.000000 & -0.000000 & 2.123500
\end{array} \\
& \begin{array}{llll}
\text { C } & 3.564160 & 4.090790 & 0.026430
\end{array} \\
& \mathrm{H} \quad 4.129670 \quad 4.949880 \quad 0.390820 \\
& \begin{array}{llll}
\text { C } & -1.047600 & -1.863550 & 3.535300
\end{array} \\
& \mathrm{H} \quad-0.078290 \quad-2.065410 \quad 3.992060
\end{aligned}
$$




\begin{tabular}{|c|c|c|c|}
\hline $\mathrm{C}$ & -3.380930 & -2.426350 & 3.357630 \\
\hline $\mathrm{H}$ & -4.233300 & -3.043940 & 3.646010 \\
\hline $\mathrm{C}$ & 0.893290 & 3.687610 & 2.590580 \\
\hline$H$ & 1.756830 & 4.324950 & 2.395450 \\
\hline$C$ & 1.303510 & -0.647100 & 2.579630 \\
\hline $\mathrm{C}$ & 2.303920 & 4.271650 & -0.524230 \\
\hline UI & 1.882230 & 5.277540 & -0.586870 \\
\hline $\mathrm{C}$ & -0.257020 & 4.226980 & 3.147830 \\
\hline $\mathrm{H}$ & -0.311060 & 5.289100 & 3.394120 \\
\hline $\mathrm{C}$ & 3.100460 & -0.928000 & 4.182890 \\
\hline $\mathrm{H}$ & 3.626410 & -0.565270 & 5.069290 \\
\hline $\mathrm{C}$ & 2.823050 & -2.544260 & 2.458440 \\
\hline $\mathrm{H}$ & 3.128720 & -3.465130 & 1.957250 \\
\hline $\mathrm{C}$ & -5.213590 & -1.782880 & -0.632920 \\
\hline $\mathrm{H}$ & -6.252890 & -2.051310 & -0.433620 \\
\hline $\mathrm{C}$ & -3.528250 & -1.391900 & 2.442690 \\
\hline $\mathrm{H}$ & -4.505080 & -1.191320 & 2.001270 \\
\hline $\mathrm{C}$ & -1.761350 & -1.444430 & -3.726860 \\
\hline $\mathrm{H}$ & -2.012750 & -2.487900 & -3.489920 \\
\hline $\mathrm{H}$ & -1.310730 & -1.414120 & -4.725140 \\
\hline $\mathrm{H}$ & -2.712050 & -0.891620 & -3.757730 \\
\hline $\mathrm{C}$ & 2.370440 & 1.050420 & -3.509730 \\
\hline $\mathrm{H}$ & 2.182900 & 2.122990 & -3.675170 \\
\hline $\mathrm{H}$ & 2.354470 & 0.549790 & -4.484050 \\
\hline $\mathrm{H}$ & 3.381760 & 0.971470 & -3.085960 \\
\hline $\mathrm{C}$ & -4.257760 & -2.773810 & -0.730900 \\
\hline $\mathrm{H}$ & -4.543600 & -3.820270 & -0.603730 \\
\hline$C$ & -2.452980 & -0.632760 & 2.067840 \\
\hline $\mathrm{H}$ & -2.600140 & 0.168400 & 1.343120 \\
\hline $\mathrm{C}$ & -1.328230 & 3.389290 & 3.392750 \\
\hline 1 & -2.241160 & 3.784830 & 3.844660 \\
\hline
\end{tabular}




$\begin{array}{lrrr}\mathrm{C} & -2.127180 & -2.641300 & 3.911130 \\ \mathrm{H} & -1.983720 & -3.434730 & 4.648650 \\ \mathrm{C} & -4.861500 & -0.469570 & -0.778380 \\ \mathrm{H} & -5.624860 & 0.308160 & -0.693570 \\ \mathrm{C} & 4.104210 & 2.825530 & 0.095390 \\ \mathrm{H} & 5.102540 & 2.687880 & 0.518060 \\ \mathrm{H} & 0.561680 & 3.321170 & -1.403180 \\ \mathrm{H} & 3.819840 & 0.707140 & -0.281310 \\ \mathrm{H} & -3.257970 & 0.948940 & -1.157380 \\ \mathrm{H} & -2.151060 & -3.233880 & -1.056940\end{array}$

Optimized, Truncated Structure for 2
$\begin{array}{llll}\text { Fe } & -0.000000 & 0.000000 & 0.000000\end{array}$
$\begin{array}{llll}\text { O } & 0.870430 & 0.799950 & 1.404460\end{array}$
$\mathrm{N} \quad 0.794430 \quad 1.053570 \quad-1.504760$
$\mathrm{N} \quad-1.076220 \quad-1.214980 \quad-1.070730$
$\begin{array}{llll}\text { C } & 1.082640 & 1.613680 & -3.864170\end{array}$
$\mathrm{H} \quad 1.093430 \quad 2.693520 \quad-3.657930$
$\mathrm{H} \quad 0.584650 \quad 1.441860 \quad-4.825130$
$\mathrm{H} \quad 2.134540 \quad 1.305010 \quad-3.964960$
$\begin{array}{llll}\text { C } & 0.414960 & 0.851270 & -2.768570\end{array}$
$\begin{array}{llll}\text { C } & -0.554450 & -0.086880 & -3.140510\end{array}$
$\mathrm{H} \quad-0.783350 \quad-0.101760 \quad-4.204920$
C $\quad-1.206650 \quad-1.060120 \quad-2.392830$
C $\quad-2.061140 \quad-2.031350 \quad-3.167930$
$\mathrm{H} \quad-3.041690 \quad-2.174520 \quad-2.691990$
$\mathrm{H} \quad-1.584550 \quad-3.021960 \quad-3.213460$
$\mathrm{H} \quad-2.212880 \quad-1.678380 \quad-4.193690$
$\begin{array}{llll}\text { C } & 1.862400 & 1.980620 & -1.221490\end{array}$
$\begin{array}{llll}\text { C } & 3.187410 & 1.508490 & -1.236280\end{array}$
$\begin{array}{llll}\text { C } & 4.193040 & 2.424180 & -0.912020\end{array}$ 


$\begin{array}{lrrr}\mathrm{H} & 5.231500 & 2.082030 & -0.917370 \\ \mathrm{C} & 3.915920 & 3.722390 & -0.614730 \\ \mathrm{H} & 4.727710 & 4.412200 & -0.375040 \\ \mathrm{C} & 2.609420 & 4.165960 & -0.579480 \\ \mathrm{H} & 2.388580 & 5.203940 & -0.321850 \\ \mathrm{C} & 1.561680 & 3.297800 & -0.881900 \\ \mathrm{C} & -1.624570 & -2.392420 & -0.450750 \\ \mathrm{C} & -2.849870 & -2.310160 & 0.249060 \\ \mathrm{C} & -3.336220 & -3.467470 & 0.843200 \\ \mathrm{H} & -4.287480 & -3.427340 & 1.379590 \\ \mathrm{C} & -2.638220 & -4.661620 & 0.782050 \\ \mathrm{H} & -3.034610 & -5.553300 & 1.269760 \\ \mathrm{C} & -1.434430 & -4.711560 & 0.110290 \\ \mathrm{H} & -1.372200 & -2.283610 & 2.637430 \\ \mathrm{H} & -0.878750 & -5.651490 & 0.068760 \\ \mathrm{C} & -0.915050 & -3.595580 & -0.526760 \\ \mathrm{C} & -0.000000 & -0.000000 & 2.072940 \\ \mathrm{C} & -1.110500 & 0.701250 & 2.763590 \\ \mathrm{C} & -1.792930 & 1.757000 & 2.165070 \\ \mathrm{H} & -1.547420 & 2.031420 & 1.134810 \\ \mathrm{C} & -2.780470 & 2.449740 & 2.834850 \\ \mathrm{H} & -3.311460 & 3.261650 & 2.334580 \\ \mathrm{C} & -3.086030 & 2.114940 & 4.146810 \\ \mathrm{H} & -3.863260 & 2.662900 & 4.683360 \\ \mathrm{H} & -2.408430 & 1.091220 & 4.764660 \\ \mathrm{H} & -3.516170 & 3.396770\end{array}$




$\begin{array}{lrrr}\mathrm{H} & -0.470050 & -4.360260 & 3.580310 \\ \mathrm{C} & 1.543230 & -3.625390 & 3.697690 \\ \mathrm{H} & 1.938310 & -4.549660 & 4.123680 \\ \mathrm{C} & 2.379630 & -2.565240 & 3.442020 \\ \mathrm{H} & 3.444810 & -2.647210 & 3.673390 \\ \mathrm{C} & 1.896880 & -1.391830 & 2.901310 \\ \mathrm{H} & 2.561550 & -0.552200 & 2.697030 \\ \mathrm{H} & 3.405070 & 0.469530 & -1.480680 \\ \mathrm{H} & 0.523340 & 3.629580 & -0.862090 \\ \mathrm{H} & -3.387970 & -1.363840 & 0.299950 \\ \mathrm{H} & 0.035990 & -3.632840 & -1.058480\end{array}$

Full Structure of 2 for SORCI and CASSCF
$\begin{array}{llll}\text { Fe } & 0.000000 & 0.000000 & 0.000000\end{array}$
$\begin{array}{llll}\text { O } & -0.820530 & 0.526180 & -1.555620\end{array}$
$\begin{array}{llll}\mathrm{N} & -1.334090 & 0.705430 & 1.314540\end{array}$
$\mathrm{N} \quad 1.201140 \quad-0.845390 \quad 1.274160$
$\begin{array}{llll}\text { C } & -2.188660 & 1.076960 & 3.571810\end{array}$
$\begin{array}{llll}\mathrm{H} & -2.461160 & 1.983130 & 3.321510\end{array}$
$\mathrm{H} \quad-1.831210 \quad 1.084380 \quad 4.484300$
$\begin{array}{llll}\mathrm{H} & -2.963670 & 0.478420 & 3.528420\end{array}$
$\begin{array}{llll}\text { C } & -1.135500 & 0.595880 & 2.630100\end{array}$
$\begin{array}{llll}\text { C } & -0.000000 & 0.000000 & 3.190260\end{array}$
$\begin{array}{llll}\mathrm{H} & 0.068310 & 0.087190 & 4.134040\end{array}$
$\begin{array}{llll}\text { C } & 1.044760 & -0.696180 & 2.594090\end{array}$
$\begin{array}{llll}\text { C } & 2.013510 & -1.371420 & 3.532060\end{array}$
H $\quad 2.931380 \quad-1.180080 \quad 3.249010$
$\mathrm{H} \quad 1.862880 \quad-2.339260 \quad 3.516410$
H $\quad 1.874940 \quad-1.035330 \quad 4.442580$
$\begin{array}{llll}\text { C } & -2.573450 & 1.257160 & 0.824830\end{array}$
$\begin{array}{llll}\text { C } & -3.663760 & 0.391810 & 0.621970\end{array}$ 


\begin{tabular}{|c|c|c|c|}
\hline $\mathrm{C}$ & -4.834260 & 0.953580 & 0.103060 \\
\hline & -5.583920 & 0.390970 & -0.057230 \\
\hline $\mathrm{C}$ & -4.932900 & 2.281170 & -0.176790 \\
\hline & -5.753440 & 2.636090 & -0.498740 \\
\hline C & -3.847320 & 3.114200 & 0.003500 \\
\hline $\mathrm{H}$ & -3.917900 & 4.033840 & -0.216980 \\
\hline $\mathrm{C}$ & -2.648700 & 2.611410 & 0.506930 \\
\hline $\mathrm{C}$ & -3.600070 & -1.092490 & 0.893370 \\
\hline $\mathrm{H}$ & -2.749080 & -1.273980 & 1.386870 \\
\hline $\mathrm{C}$ & -3.545260 & -1.870880 & -0.422250 \\
\hline $\mathrm{H}$ & -2.761580 & -1.586740 & -0.937020 \\
\hline $\mathrm{H}$ & -4.358210 & -1.693450 & -0.939980 \\
\hline $\mathrm{H}$ & -3.480410 & -2.830530 & -0.231460 \\
\hline $\mathrm{C}$ & -4.749820 & -1.583250 & 1.762610 \\
\hline $\mathrm{H}$ & -4.724180 & -1.123910 & 2.627550 \\
\hline $\mathrm{H}$ & -4.664280 & -2.550090 & 1.903200 \\
\hline $\mathrm{H}$ & -5.601100 & -1.391770 & 1.315720 \\
\hline $\mathrm{C}$ & -1.460920 & 3.552900 & 0.652030 \\
\hline $\mathrm{H}$ & -0.651900 & 2.998120 & 0.849910 \\
\hline $\mathrm{C}$ & -1.187710 & 4.338740 & -0.619700 \\
\hline $\mathrm{H}$ & -1.097260 & 3.720980 & -1.374130 \\
\hline $\mathrm{H}$ & -0.357450 & 4.848790 & -0.515190 \\
\hline $\mathrm{H}$ & -1.931380 & 4.952540 & -0.789580 \\
\hline $\mathrm{C}$ & -1.662710 & 4.523450 & 1.824660 \\
\hline $\mathrm{H}$ & -1.689400 & 4.019300 & 2.664130 \\
\hline $\mathrm{H}$ & -2.507590 & 5.007870 & 1.705420 \\
\hline $\mathrm{H}$ & -0.920750 & 5.161660 & 1.851000 \\
\hline C & 2.191130 & -1.771720 & 0.791210 \\
\hline 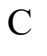 & 3.430000 & -1.287550 & 0.313020 \\
\hline$c$ & 4.351320 & -2.215050 & -0.155810 \\
\hline $\mathrm{H}$ & 5.197180 & -1.913850 & -0.466250 \\
\hline
\end{tabular}




\begin{tabular}{|c|c|c|c|}
\hline $\mathrm{C}$ & 4.065410 & -3.569450 & -0.184400 \\
\hline $\mathrm{H}$ & 4.707490 & -4.187800 & -0.514500 \\
\hline$C$ & 2.841310 & -4.015360 & 0.269030 \\
\hline $\mathrm{H}$ & 2.644070 & -4.945790 & 0.235520 \\
\hline$C$ & 1.894740 & -3.138720 & 0.775490 \\
\hline C & 3.782060 & 0.176990 & 0.305000 \\
\hline $\mathrm{H}$ & 2.936570 & 0.702440 & 0.400530 \\
\hline $\mathrm{C}$ & 4.458410 & 0.588970 & -1.007430 \\
\hline $\mathrm{H}$ & 3.960210 & 0.213310 & -1.764320 \\
\hline $\mathrm{H}$ & 5.377330 & 0.249900 & -1.022060 \\
\hline $\mathrm{H}$ & 4.468810 & 1.567260 & -1.077150 \\
\hline $\mathrm{C}$ & 4.696550 & 0.526380 & 1.473710 \\
\hline $\mathrm{H}$ & 4.219050 & 0.378200 & 2.317050 \\
\hline $\mathrm{H}$ & 4.961660 & 1.466830 & 1.409790 \\
\hline $\mathrm{H}$ & 5.494960 & -0.041630 & 1.445100 \\
\hline $\mathrm{C}$ & 0.542320 & -3.665320 & 1.245140 \\
\hline $\mathrm{H}$ & 0.145190 & -2.986420 & 1.863070 \\
\hline $\mathrm{C}$ & -0.392560 & -3.821960 & 0.061330 \\
\hline $\mathrm{H}$ & -0.529900 & -2.950800 & -0.364620 \\
\hline $\mathrm{H}$ & -1.254140 & -4.173130 & 0.368410 \\
\hline $\mathrm{H}$ & 0.000890 & -4.441590 & -0.587030 \\
\hline$C$ & 0.660430 & -4.984120 & 2.005130 \\
\hline $\mathrm{H}$ & 1.312690 & -4.884760 & 2.729120 \\
\hline $\mathrm{H}$ & 0.957320 & -5.689990 & 1.391500 \\
\hline $\mathrm{H}$ & -0.211560 & -5.222010 & 2.381230 \\
\hline $\mathrm{C}$ & 0.359560 & 0.060770 & -2.040610 \\
\hline C & 1.294790 & 1.095800 & -2.546590 \\
\hline$C$ & 1.495330 & 2.294650 & -1.867550 \\
\hline 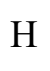 & 1.059380 & 2.436280 & -1.035220 \\
\hline$\tau$ & 2.314880 & 3.282300 & -2.374120 \\
\hline $\mathrm{H}$ & 2.454180 & 4.083860 & -1.884640 \\
\hline
\end{tabular}




$\begin{array}{lrrr}\mathrm{C} & 2.933000 & 3.099290 & -3.603400 \\ \mathrm{H} & 3.494170 & 3.776490 & -3.960260 \\ \mathrm{C} & 2.730240 & 1.932920 & -4.301500 \\ \mathrm{H} & 3.150580 & 1.812740 & -5.144790 \\ \mathrm{C} & 1.925710 & 0.938490 & -3.793680 \\ \mathrm{H} & 1.795530 & 0.140070 & -4.290310 \\ \mathrm{C} & 0.368380 & -1.287900 & -2.634270 \\ \mathrm{C} & 1.527110 & -2.051970 & -2.708200 \\ \mathrm{H} & 2.339510 & -1.705550 & -2.356370 \\ \mathrm{C} & 1.511780 & -3.298600 & -3.282430 \\ \mathrm{H} & 2.311510 & -3.812010 & -3.316360 \\ \mathrm{C} & 0.342950 & -3.816800 & -3.809500 \\ \mathrm{H} & 0.340700 & -4.672940 & -4.220740 \\ \mathrm{C} & -0.815720 & -3.081720 & -3.732050 \\ \mathrm{H} & -1.620450 & -3.438930 & -4.086740 \\ \mathrm{C} & -0.828530 & -1.832390 & -3.147830 \\ \mathrm{H} & -1.639390 & -1.340320 & -3.091340\end{array}$

\section{Optimized, Truncated Structure of 3 for TD-DFT}

$\begin{array}{lrrr}\mathrm{Fe} & 0.000000 & 0.000000 & 0.000000 \\ \mathrm{C} & -1.775740 & -1.559020 & -3.631860 \\ \mathrm{H} & -1.714570 & -2.639840 & -3.432470 \\ \mathrm{H} & -1.482930 & -1.381930 & -4.673040 \\ \mathrm{H} & -2.832940 & -1.276470 & -3.517260 \\ \mathrm{C} & -2.142680 & -1.732070 & -0.852790 \\ \mathrm{C} & 2.121900 & 1.814540 & -0.701130 \\ \mathrm{C} & 0.000000 & -0.000000 & 2.002830 \\ \mathrm{H} & -0.810570 & 0.648510 & 2.379680 \\ \mathrm{H} & 0.954570 & 0.380120 & 2.403520\end{array}$




\begin{tabular}{|c|c|c|c|}
\hline & -0.168010 & -1.012380 & 2.408560 \\
\hline & -0.894170 & -0.784300 & -2.688970 \\
\hline & -3.425320 & -1.182370 & -0.701080 \\
\hline & 1.763680 & 3.142850 & -0.418570 \\
\hline & 0.085730 & 0.036390 & -3.258110 \\
\hline & 0.117850 & 0.039600 & -4.346860 \\
\hline C & -4.396290 & -1.940350 & -0.051760 \\
\hline & -5.393930 & -1.516730 & 0.086920 \\
\hline C & 2.682280 & 3.930480 & 0.286680 \\
\hline $\mathrm{H}$ & 2.418080 & 4.962800 & 0.528750 \\
\hline $\mathrm{C}$ & 1.034960 & 0.850510 & -2.627370 \\
\hline $\mathrm{C}$ & -4.115770 & -3.198480 & 0.428680 \\
\hline $\mathrm{H}$ & -4.888150 & -3.769360 & 0.947530 \\
\hline C & 3.905260 & 3.429780 & 0.670250 \\
\hline $\mathrm{H}$ & 4.604800 & 4.065420 & 1.216690 \\
\hline $\mathrm{C}$ & 1.959700 & 1.659110 & -3.505380 \\
\hline $\mathrm{H}$ & 1.823960 & 2.737410 & -3.333920 \\
\hline $\mathrm{H}$ & 1.772150 & 1.451850 & -4.565180 \\
\hline $\mathrm{H}$ & 3.013830 & 1.434310 & -3.284470 \\
\hline $\mathrm{C}$ & -2.868310 & -3.741570 & 0.244710 \\
\hline $\mathrm{H}$ & -2.653200 & -4.746750 & 0.615760 \\
\hline $\mathrm{C}$ & 4.245460 & 2.133390 & 0.375340 \\
\hline H & 5.216370 & 1.741910 & 0.689220 \\
\hline C & -1.852100 & -3.028980 & -0.398530 \\
\hline $\mathrm{C}$ & 3.362840 & 1.288360 & -0.302020 \\
\hline $\mathrm{N}$ & -1.077970 & -0.913970 & -1.375800 \\
\hline $\mathrm{N}$ & 1.146830 & 0.939320 & -1.301850 \\
\hline $\mathrm{H}$ & -3.631980 & -0.173840 & -1.059480 \\
\hline $\mathrm{H}$ & 0.788870 & 3.525970 & -0.717140 \\
\hline $\mathrm{H}$ & -0.852260 & -3.443760 & -0.526310 \\
\hline & 3.613830 & 0.250410 & -0.520280 \\
\hline
\end{tabular}




\section{Optimized, Truncated Structure of 4 for TD-DFT}

$$
\begin{aligned}
& \begin{array}{llll}
\text { Fe } & 0.000000 & 0.000000 & 0.000000
\end{array} \\
& \begin{array}{llll}
\text { C } & -0.000000 & -0.000000 & 2.036910
\end{array} \\
& \begin{array}{llll}
\mathrm{H} & 0.287140 & -0.986350 & 2.440760
\end{array} \\
& \begin{array}{llll}
\mathrm{H} & 0.647690 & 0.777350 & 2.479060
\end{array} \\
& \begin{array}{llll}
\mathrm{H} & -1.042290 & 0.218870 & 2.331620
\end{array} \\
& \begin{array}{llll}
\mathrm{N} & 0.513630 & -1.400500 & -1.227490
\end{array} \\
& \begin{array}{llll}
\mathrm{N} & -0.366230 & 1.393280 & -1.267970
\end{array} \\
& \begin{array}{llll}
\text { C } & 1.099150 & -2.308400 & -3.490750
\end{array} \\
& \mathrm{H} \quad 1.937160 \quad-2.874100 \quad-3.055180 \\
& \text { H } \quad 1.420000 \quad-1.894210 \quad-4.454700 \\
& \mathrm{H} \quad 0.295010 \quad-3.036230 \quad-3.687500 \\
& \begin{array}{llll}
\text { C } & 0.644050 & -1.179080 & -2.564380
\end{array} \\
& \begin{array}{llll}
\text { C } & 0.437500 & 0.079760 & -3.156350
\end{array} \\
& \text { H } \quad 0.651450 \quad 0.127220 \quad-4.224840 \\
& \begin{array}{llll}
\text { C } & -0.033900 & 1.258030 & -2.593590
\end{array} \\
& \begin{array}{llll}
\text { C } & -0.155160 & 2.483760 & -3.487750
\end{array} \\
& \begin{array}{llll}
\mathrm{H} & -1.198010 & 2.823830 & -3.585610
\end{array} \\
& \mathrm{H} \quad 0.223750 \quad 2.258550 \quad-4.492590 \\
& \begin{array}{llll}
\mathrm{H} & 0.415860 & 3.336220 & -3.087020
\end{array} \\
& \begin{array}{llll}
\text { C } & 0.639770 & -2.606600 & -0.675150
\end{array} \\
& \begin{array}{llll}
\text { C } & -0.346540 & -3.590860 & -0.922360
\end{array} \\
& \begin{array}{llll}
\text { C } & -0.259610 & -4.831290 & -0.300940
\end{array} \\
& \text { H } \quad-1.024220 \quad-5.589670 \quad-0.486900 \\
& \begin{array}{llll}
\text { C } & 0.782050 & -5.089780 & 0.592490
\end{array} \\
& \begin{array}{llll}
\mathrm{H} & 0.837140 & -6.048990 & 1.110320
\end{array} \\
& \begin{array}{llll}
\text { C } & 1.741650 & -4.121930 & 0.816820
\end{array} \\
& \text { H } \quad 2.560330 \quad-4.340500 \quad 1.508920 \\
& \begin{array}{llll}
\text { C } & 1.722450 & -2.896460 & 0.170080
\end{array} \\
& \begin{array}{llll}
\text { C } & -0.779340 & 2.545750 & -0.804130
\end{array}
\end{aligned}
$$




$$
\begin{aligned}
& \begin{array}{llll}
\text { C } & -2.103880 & 2.913120 & -0.982700
\end{array} \\
& \begin{array}{llll}
\text { C } & -2.754650 & 3.973510 & -0.359200
\end{array} \\
& \text { H } \quad-3.828690 \quad 4.120470 \quad-0.478840 \\
& \begin{array}{llll}
\text { C } & -1.944640 & 4.907000 & 0.281760
\end{array} \\
& \begin{array}{llll}
\mathrm{H} & -2.374290 & 5.801680 & 0.738800
\end{array} \\
& \begin{array}{llll}
\text { C } & -0.595920 & 4.642070 & 0.422550
\end{array} \\
& \begin{array}{llll}
\mathrm{H} & 0.039070 & 5.354630 & 0.955260
\end{array} \\
& \begin{array}{llll}
\text { C } & -0.015190 & 3.487340 & -0.090190
\end{array} \\
& \begin{array}{llll}
\text { K } & -0.997450 & -0.501750 & 4.951920
\end{array} \\
& \begin{array}{llll}
\text { O } & -3.186540 & -1.354000 & 3.331290
\end{array} \\
& \begin{array}{llll}
\text { O } & -0.925020 & -3.291940 & 4.230950
\end{array} \\
& \begin{array}{llll}
\text { O } & 0.924840 & -2.374010 & 6.236900
\end{array} \\
& \begin{array}{llll}
\text { O } & 1.077490 & 0.426060 & 6.445270
\end{array} \\
& \begin{array}{llll}
\text { O } & -0.997730 & 2.102850 & 6.087340
\end{array} \\
& \begin{array}{llll}
\text { O } & -2.960080 & 1.093630 & 4.261440
\end{array} \\
& \begin{array}{llll}
\text { C } & -2.805660 & -2.670480 & 2.792390
\end{array} \\
& \mathrm{H} \quad-3.709210 \quad-3.200410 \quad 2.442510 \\
& \mathrm{H} \quad-2.205930 \quad-2.436230 \quad 1.892260 \\
& \begin{array}{llll}
\text { C } & -2.090180 & -3.654570 & 3.605850
\end{array} \\
& \text { H } \quad-2.788060 \quad-4.070730 \quad 4.368860 \\
& \text { Н } \quad-1.913060 \quad-4.486470 \quad 2.888900 \\
& \begin{array}{llll}
\text { C } & -0.230300 & -3.876470 & 5.212950
\end{array} \\
& \mathrm{H} \quad-0.237850 \quad-4.983840 \quad 5.025940 \\
& \mathrm{H} \quad-0.794280 \quad-3.802080 \quad 6.173510 \\
& \begin{array}{llll}
\text { C } & 1.122340 & -3.549490 & 5.539150
\end{array} \\
& \mathrm{H} \quad 1.559250 \quad-4.342560 \quad 6.173490 \\
& \text { H } \quad 1.783250 \quad-3.393340 \quad 4.663660 \\
& \begin{array}{llll}
\text { C } & 2.092790 & -1.731580 & 6.457470
\end{array} \\
& \begin{array}{llll}
\mathrm{H} & 2.637980 & -1.587560 & 5.496370
\end{array} \\
& \begin{array}{llll}
\mathrm{H} & 2.777760 & -2.330610 & 7.097810
\end{array} \\
& \begin{array}{llll}
\text { C } & 1.924780 & -0.409390 & 7.124780
\end{array}
\end{aligned}
$$




\begin{tabular}{lrrr}
$\mathrm{H}$ & 1.539050 & -0.597660 & 8.147040 \\
$\mathrm{H}$ & 2.933370 & 0.037260 & 7.243970 \\
$\mathrm{C}$ & 1.023200 & 1.513680 & 7.321930 \\
$\mathrm{H}$ & 1.900470 & 2.159120 & 7.112170 \\
$\mathrm{H}$ & 1.116230 & 1.197000 & 8.371640 \\
$\mathrm{C}$ & -0.230840 & 2.266510 & 7.226810 \\
$\mathrm{H}$ & -0.871080 & 1.976680 & 8.086060 \\
$\mathrm{H}$ & -0.001900 & 3.338580 & 7.402030 \\
$\mathrm{C}$ & -1.816840 & 2.844640 & 5.209330 \\
$\mathrm{H}$ & -1.255620 & 2.924470 & 4.258650 \\
$\mathrm{H}$ & -1.903260 & 3.888050 & 5.581750 \\
$\mathrm{C}$ & -3.158370 & 2.387490 & 4.855800 \\
$\mathrm{H}$ & -3.875530 & 2.305370 & 5.690660 \\
$\mathrm{H}$ & -3.543060 & 3.104750 & 4.111060 \\
$\mathrm{C}$ & -4.037730 & 0.939380 & 3.466900 \\
$\mathrm{H}$ & -3.900530 & 1.547170 & 2.541810 \\
$\mathrm{H}$ & -4.972680 & 1.305910 & 3.932490 \\
$\mathrm{C}$ & -4.223380 & -0.447230 & 3.177380 \\
$\mathrm{H}$ & -5.051910 & -0.815840 & 3.825750 \\
$\mathrm{H}$ & -4.654520 & -0.527470 & 2.154820 \\
$\mathrm{H}$ & -1.177290 & -3.348670 & -1.586890 \\
$\mathrm{H}$ & 2.483890 & -2.136590 & 0.347730 \\
\hline & -2.725330 & 2.201490 & -1.539490 \\
$\mathrm{H}$ & 1.046820 & 3.289820 & 0.080590
\end{tabular}

\section{Optimized Truncated Structure of 5 for TD-DFT}

$\begin{array}{lrrr}\mathrm{Fe} & 0.000000 & 0.000000 & 0.000000 \\ \mathrm{~N} & -1.435360 & 0.017150 & 1.353610 \\ \mathrm{~N} & 1.455130 & -0.003590 & 1.357390 \\ \mathrm{C} & 3.510860 & -1.100380 & 0.594130 \\ \mathrm{C} & -1.256920 & 0.017020 & 2.676350\end{array}$



$\begin{array}{llll}\text { C } & 2.804780 & 0.075410 & 0.852660\end{array}$
$\begin{array}{llll}\text { C } & 3.345100 & 1.338040 & 0.563250\end{array}$
$\begin{array}{llll}\text { C } & 1.265300 & 0.019190 & 2.679050\end{array}$
$\begin{array}{llll}\text { C } & -0.000000 & 0.000000 & 3.277830\end{array}$
$\mathrm{H} \quad-0.001020 \quad 0.001580 \quad 4.366640$
$\begin{array}{llll}\text { C } & -2.766250 & 0.103780 & 0.824430\end{array}$
$\begin{array}{llll}\text { C } & -1.248730 & 0.280690 & -3.020670\end{array}$
$\begin{array}{llll}\text { C } & -2.455360 & 0.021950 & 3.596070\end{array}$
$\begin{array}{llll}\mathrm{H} & -3.098440 & 0.894500 & 3.407450\end{array}$
$\begin{array}{llll}\mathrm{H} & -2.137980 & 0.043310 & 4.644420\end{array}$
$\mathrm{H} \quad-3.079150 \quad-0.870410 \quad 3.438530$
$\begin{array}{llll}\text { C } & 2.460780 & 0.074380 & 3.604520\end{array}$
H $\quad 3.125840 \quad-0.787360 \quad 3.446800$
$\begin{array}{llll}\mathrm{H} & 2.137790 & 0.078830 & 4.651720\end{array}$
$\begin{array}{llll}\mathrm{H} & 3.062160 & 0.976690 & 3.420710\end{array}$
$\begin{array}{llll}\text { C } & -3.500430 & -1.075810 & 0.603580\end{array}$
$\begin{array}{llll}\text { C } & -3.268150 & 1.359200 & 0.457870\end{array}$
$\begin{array}{llll}\text { C } & 4.774620 & -0.995860 & 0.012270\end{array}$
$\begin{array}{llll}\mathrm{H} & 5.340140 & -1.904500 & -0.206500\end{array}$
$\begin{array}{llll}\text { C } & 4.608160 & 1.381840 & -0.019910\end{array}$
H $\quad 5.041760 \quad 2.355200 \quad-0.264440$
$\begin{array}{llll}\text { C } & -4.543680 & 1.412760 & -0.119310\end{array}$
$\mathrm{H} \quad-4.954470 \quad 2.383130 \quad-0.406960$
$\begin{array}{llll}\text { C } & -0.285760 & 0.120350 & -1.932810\end{array}$
$\begin{array}{llll}\text { C } & 0.949380 & 0.020230 & -1.665860\end{array}$
$\begin{array}{llll}\text { C } & -2.600660 & 0.117650 & -2.829870\end{array}$
$\mathrm{H} \quad-2.975610 \quad-0.155090 \quad-1.845060$
$\begin{array}{llll}\text { C } & -4.754910 & -0.957660 & 0.005290\end{array}$
$\begin{array}{llll}\mathrm{H} & -5.335970 & -1.863070 & -0.186010\end{array}$
$\begin{array}{llll}\text { C } & -0.817460 & 0.609110 & -4.281480\end{array}$
$\begin{array}{llll}\mathrm{H} & 0.252380 & 0.740110 & -4.462420\end{array}$ 


$$
\begin{array}{lrrr}
\mathrm{C} & -5.269490 & 0.266450 & -0.346600 \\
\mathrm{H} & -6.254080 & 0.328990 & -0.813340 \\
\mathrm{C} & 5.313860 & 0.241560 & -0.291290 \\
\mathrm{H} & 6.300700 & 0.304910 & -0.753530 \\
\mathrm{C} & -3.489620 & 0.290850 & -3.878730 \\
\mathrm{H} & -4.557940 & 0.156840 & -3.695470 \\
\mathrm{C} & -3.043160 & 0.631820 & -5.112670 \\
\mathrm{H} & -3.751670 & 0.775330 & -5.932250 \\
\mathrm{C} & -1.705810 & 0.783330 & -5.332210 \\
\mathrm{H} & -1.325480 & 1.048530 & -6.320480 \\
\mathrm{H} & 1.957530 & -0.027510 & -2.072640 \\
\mathrm{H} & 3.064870 & -2.068410 & 0.823760 \\
\mathrm{H} & 2.772460 & 2.242000 & 0.769600 \\
\mathrm{H} & -3.080560 & -2.043360 & 0.877710 \\
\mathrm{H} & -2.674470 & 2.257840 & 0.624160
\end{array}
$$

\section{Full Structure of 5 for SORCI and CASSCF}

$$
\begin{array}{lrrr}
\mathrm{Fe} & 0.000000 & 0.000000 & 0.000000 \\
\mathrm{~N} & -1.435350 & 0.017630 & 1.353610 \\
\mathrm{~N} & 1.455130 & -0.004080 & 1.357390 \\
\mathrm{C} & 3.510500 & -1.101550 & 0.594130 \\
\mathrm{C} & -1.256910 & 0.017440 & 2.676350 \\
\mathrm{C} & 2.804810 & 0.074470 & 0.852660 \\
\mathrm{C} & 3.345550 & 1.336930 & 0.563250 \\
\mathrm{C} & 1.265310 & 0.018760 & 2.679050 \\
\mathrm{C} & 0.000000 & -0.000000 & 3.277830 \\
\mathrm{H} & -0.001260 & -0.029130 & 4.207520 \\
\mathrm{C} & -2.766210 & 0.104700 & 0.824430 \\
\mathrm{C} & -1.248630 & 0.281110 & -3.020670 \\
\mathrm{C} & -2.455350 & 0.022760 & 3.596070 \\
\mathrm{H} & -3.059050 & 0.717460 & 3.330240
\end{array}
$$




$\begin{array}{lrrr}\mathrm{H} & -2.165110 & 0.176830 & 4.498410 \\ \mathrm{H} & -2.900860 & -0.826770 & 3.544670 \\ \mathrm{C} & 2.460810 & 0.073560 & 3.604520 \\ \mathrm{H} & 3.039260 & -0.672530 & 3.424730 \\ \mathrm{H} & 2.162200 & 0.034780 & 4.516780 \\ \mathrm{H} & 2.940490 & 0.892300 & 3.459700 \\ \mathrm{C} & -3.500780 & -1.074640 & 0.603580 \\ \mathrm{C} & -3.267700 & 1.360290 & 0.457870 \\ \mathrm{C} & 2.921970 & -2.472050 & 0.877630 \\ \mathrm{H} & 2.124110 & -2.340930 & 1.431670 \\ \mathrm{C} & 4.774290 & -0.997460 & 0.012270 \\ \mathrm{H} & 5.259270 & -1.768590 & -0.173730 \\ \mathrm{C} & 4.608620 & 1.380300 & -0.019910 \\ \mathrm{H} & -1.549350 & 2.355530 & 0.945150 \\ \mathrm{H} & 4.981380 & 2.207020 & -0.230490 \\ \mathrm{C} & -4.543210 & 1.414270 & -0.119300 \\ \mathrm{H} & -2.214780 & -2.304000 & 1.594630 \\ \mathrm{H} & -4.905160 & 2.239220 & -0.353790 \\ \mathrm{C} & -0.285720 & 0.120450 & -1.932810 \\ \mathrm{C} & 0.949390 & 0.019920 & -1.665860 \\ \mathrm{C} & 2.593410 & 2.615750 & 0.885670 \\ \mathrm{H} & 1.850480 & 2.384090 & 1.482080 \\ \mathrm{H} & -2.600620 & 0.118520 & -2.829870 \\ \mathrm{H} & -2.921150 & -0.110890 & -1.987890 \\ \mathrm{H} & -2.870080 & -3.396790 & 1.647030 \\ \mathrm{H} & -3.133660 & -0.411980\end{array}$




\begin{tabular}{|c|c|c|c|}
\hline U & 1.864570 & -2.553160 & -0.873870 \\
\hline & 2.039680 & -3.963770 & -0.209890 \\
\hline & 3.240940 & -3.299820 & -0.966840 \\
\hline & -4.755230 & -0.956080 & 0.005290 \\
\hline & -5.254770 & -1.721830 & -0.159220 \\
\hline$C$ & -0.817250 & 0.609380 & -4.281480 \\
\hline & 0.093640 & 0.717120 & -4.435570 \\
\hline $\mathrm{C}$ & 3.474260 & 3.625550 & 1.612210 \\
\hline$\theta$ & 3.851370 & 3.216840 & 2.394270 \\
\hline $\mathrm{H}$ & 2.946120 & 4.383850 & 1.870170 \\
\hline $\mathrm{H}$ & 4.180530 & 3.911810 & 1.027930 \\
\hline $\mathrm{C}$ & -5.269400 & 0.268210 & -0.346600 \\
\hline $\mathrm{H}$ & -6.111500 & 0.323730 & -0.739110 \\
\hline $\mathrm{C}$ & 5.313940 & 0.239780 & -0.291290 \\
\hline $\mathrm{H}$ & 6.156570 & 0.295110 & -0.680010 \\
\hline $\mathrm{C}$ & -3.014300 & 3.524120 & 1.726640 \\
\hline $\mathrm{H}$ & -2.460100 & 4.301770 & 1.821890 \\
\hline $\mathrm{H}$ & -3.042620 & 3.048340 & 2.560050 \\
\hline $\mathrm{H}$ & -3.902810 & 3.793230 & 1.480570 \\
\hline$C$ & -3.489520 & 0.292010 & -3.878720 \\
\hline $\mathrm{H}$ & -4.401080 & 0.173050 & -3.734870 \\
\hline $\mathrm{C}$ & 2.001030 & 3.260930 & -0.372700 \\
\hline $\mathrm{H}$ & 2.712720 & 3.522870 & -0.962500 \\
\hline $\mathrm{H}$ & 1.489070 & 4.031940 & -0.125280 \\
\hline $\mathrm{H}$ & 1.432750 & 2.629920 & -0.819560 \\
\hline $\mathrm{C}$ & -3.042940 & 0.632840 & -5.112670 \\
\hline $\mathrm{H}$ & -3.646210 & 0.763680 & -5.807950 \\
\hline $\mathrm{C}$ & -1.705540 & 0.783900 & -5.332200 \\
\hline 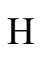 & -1.391580 & 1.002240 & -6.180690 \\
\hline $\mathrm{C}$ & -3.943570 & -3.383210 & 1.561750 \\
\hline $\mathrm{H}$ & -4.327740 & -2.969640 & 2.338980 \\
\hline
\end{tabular}




$\begin{array}{lrrr}\mathrm{H} & -3.507420 & -4.199020 & 1.814070 \\ \mathrm{H} & -4.637280 & -3.572950 & 0.925800 \\ \mathrm{C} & -2.302280 & -3.076530 & -0.288310 \\ \mathrm{H} & -2.979680 & -3.233460 & -0.950160 \\ \mathrm{H} & -1.895700 & -3.912020 & -0.042800 \\ \mathrm{H} & -1.634140 & -2.487790 & -0.647430 \\ \mathrm{C} & -2.289830 & 3.411950 & -0.625590 \\ \mathrm{H} & -3.148420 & 3.724840 & -0.913790 \\ \mathrm{H} & -1.911820 & 2.845930 & -1.302390 \\ \mathrm{H} & -1.708330 & 4.160030 & -0.472560 \\ \mathrm{H} & 1.861770 & -0.054790 & -2.025300\end{array}$




\section{X-ray crystallography:}

\section{$\left[\mathrm{L}^{\mathrm{Me}} \mathrm{Fe}\left(\mathrm{CPh}_{3}\right](\mathbf{1})\right.$}

A crystal $\left(0.48 \times 0.14 \times 0.06 \mathrm{~mm}^{3}\right)$ was placed onto the tip of a $0.1 \mathrm{~mm}$ diameter glass capillary tube or fiber and mounted on a Bruker SMART APEX II CCD Platform diffractometer for a data collection at $100.0(5) \mathrm{K} .{ }^{13} \mathrm{~A}$ preliminary set of cell constants and an orientation matrix were calculated from reflections harvested from three orthogonal wedges of reciprocal space. The full data collection was carried out using MoKa radiation (graphite monochromator) with a frame time of 90 seconds and a detector distance of $3.99 \mathrm{~cm}$. A randomly oriented region of reciprocal space was surveyed: six major sections of frames were collected with $0.50^{\circ}$ steps in $\omega$ at six different $\phi$ settings and a detector position of $-38^{\circ}$ in $2 \theta$. The intensity data were corrected for absorption. ${ }^{14}$ Final cell constants were calculated from the xyz centroids of 4024 strong reflections from the actual data collection after integration. ${ }^{15}$ See Table S-1 for additional crystal and refinement information.

The structure was solved using SIR97 ${ }^{16}$ and refined using SHELXL-97. ${ }^{17}$ The space group $P 2{ }_{1} / n$ was determined based on systematic absences and intensity statistics. A direct-methods solution was calculated which provided most non-hydrogen atoms from the E-map. Full-matrix least squares / difference Fourier cycles were performed which located the remaining non-hydrogen atoms. All non-hydrogen atoms were refined with anisotropic displacement parameters. All hydrogen atoms were placed in ideal positions and refined as riding atoms with relative isotropic displacement parameters. The final full matrix least squares refinement converged to $R 1=0.0502$ $\left(F^{2}, I>2 \sigma(I)\right)$ and $w R 2=0.1307\left(F^{2}\right.$, all data).

The structure is the one suggested. The asymmetric unit contains one iron molecule and one-half molecule of cocrystallized diethyl ether solvent. The diethyl ether molecule is modeled as disordered over a special position. Unless noted otherwise all structural diagrams containing thermal displacement ellipsoids are drawn at the $50 \%$ probability level. 

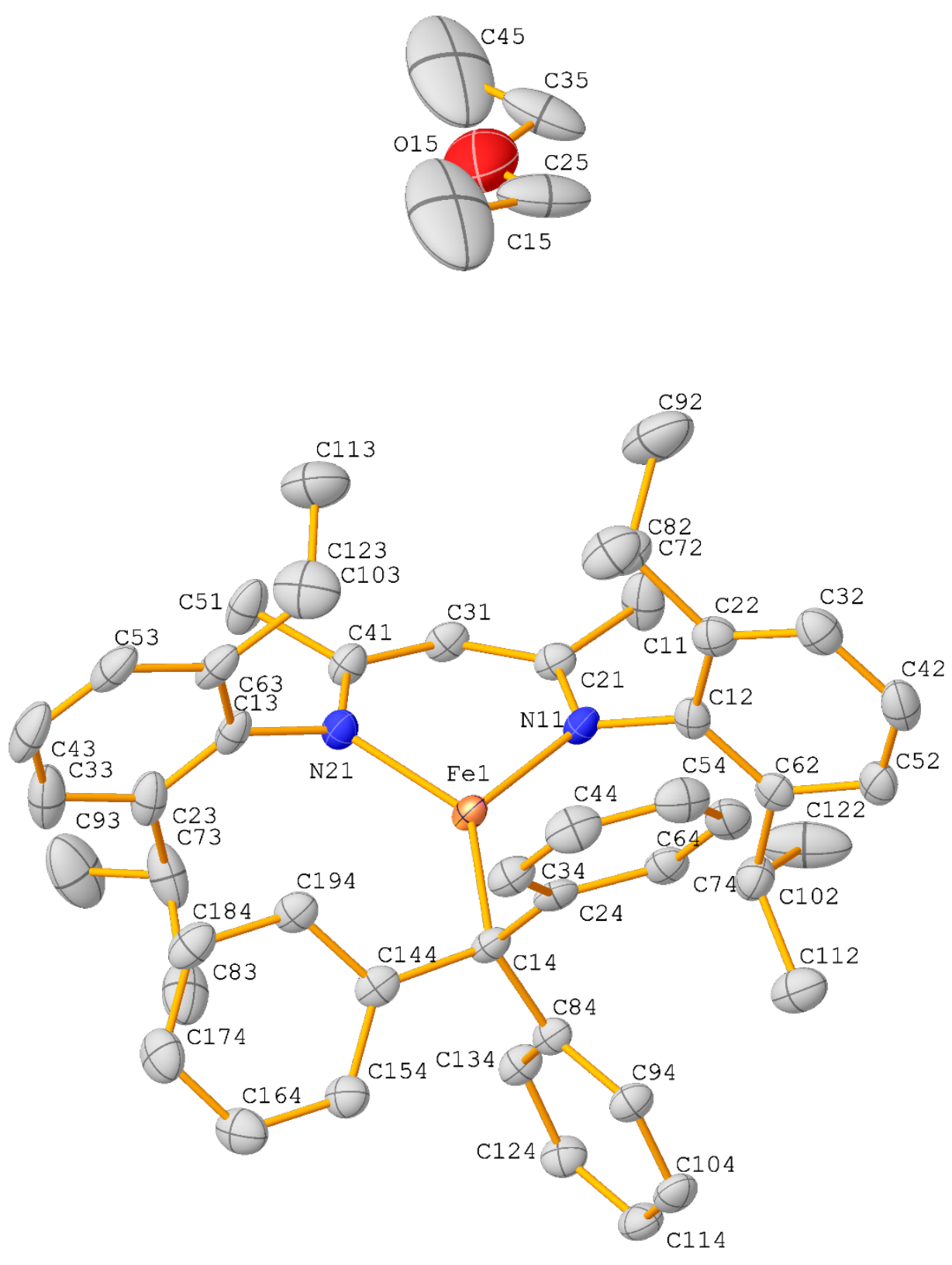

Figure S-24: The complete numbering scheme of $\mathrm{L}^{\mathrm{Me}} \mathrm{Fe}\left(\mathrm{CPh}_{3}\right)$ (1) with $50 \%$ thermal ellipsoid probability levels. The hydrogen atoms are omitted for clarity. 
Table S-1. Crystal data and structure refinement for $\mathrm{L}^{\mathrm{Me}} \mathrm{Fe}\left(\mathrm{CPh}_{3}\right]$ (1).

\begin{tabular}{|c|c|c|}
\hline Identification code & \multicolumn{2}{|l|}{ holkcm06 } \\
\hline CSD Deposition Number & \multicolumn{2}{|l|}{1944808} \\
\hline Empirical formula & \multicolumn{2}{|l|}{$\mathrm{C}_{50} \mathrm{H}_{61} \mathrm{Fe} \mathrm{N}_{2} \mathrm{O}_{0.5}$} \\
\hline Formula weight & \multicolumn{2}{|l|}{753.86} \\
\hline Temperature & \multicolumn{2}{|l|}{$100.0(5) \mathrm{K}$} \\
\hline Wavelength & \multicolumn{2}{|l|}{$0.71073 \AA$} \\
\hline Crystal system & \multicolumn{2}{|l|}{ Monoclinic } \\
\hline Space group & \multicolumn{2}{|l|}{$P 2{ }_{1} / n$} \\
\hline \multirow[t]{3}{*}{ Unit cell dimensions } & $a=19.946(4) \AA$ & $\alpha=90^{\circ}$ \\
\hline & $b=10.0572(18) \AA$ & $\beta=108.414(4)^{\circ}$ \\
\hline & $c=22.639(4) \AA$ & $\gamma=90^{\circ}$ \\
\hline Volume & \multicolumn{2}{|l|}{$4308.9(13) \AA^{3}$} \\
\hline$Z$ & \multicolumn{2}{|c|}{4} \\
\hline Density (calculated) & \multicolumn{2}{|l|}{$1.162 \mathrm{~g} / \mathrm{cm}^{3}$} \\
\hline Absorption coefficient & \multicolumn{2}{|l|}{$0.386 \mathrm{~mm}^{-1}$} \\
\hline$F(000)$ & \multicolumn{2}{|l|}{1620} \\
\hline Crystal color, morphology & \multicolumn{2}{|l|}{ orange, needle } \\
\hline Crystal size & \multicolumn{2}{|c|}{$0.48 \times 0.14 \times 0.06 \mathrm{~mm}^{3}$} \\
\hline Theta range for data collection & \multicolumn{2}{|l|}{1.90 to $24.99^{\circ}$} \\
\hline Index ranges & \multicolumn{2}{|c|}{$-23 \leq h \leq 23,-11 \leq k \leq 11,-26 \leq l \leq 26$} \\
\hline Reflections collected & \multicolumn{2}{|l|}{57089} \\
\hline Independent reflections & \multicolumn{2}{|c|}{$7554[R($ int $)=0.1431]$} \\
\hline Observed reflections & \multicolumn{2}{|l|}{5083} \\
\hline Completeness to theta $=24.99^{\circ}$ & \multicolumn{2}{|l|}{$99.7 \%$} \\
\hline Absorption correction & \multicolumn{2}{|l|}{ Multi-scan } \\
\hline Max. and min. transmission & \multicolumn{2}{|l|}{0.9772 and 0.8364} \\
\hline Refinement method & \multicolumn{2}{|c|}{ Full-matrix least-squares on $F^{2}$} \\
\hline Data / restraints / parameters & \multicolumn{2}{|l|}{7554 / 5 / 509} \\
\hline Goodness-of-fit on $F^{2}$ & \multicolumn{2}{|l|}{1.008} \\
\hline Final $R$ indices $[I>2 \operatorname{sigma}(I)]$ & \multicolumn{2}{|c|}{$R 1=0.0502, w R 2=0.1124$} \\
\hline$R$ indices (all data) & \multicolumn{2}{|c|}{$R 1=0.0891, w R 2=0.1307$} \\
\hline Largest diff. peak and hole & \multicolumn{2}{|c|}{0.522 and -0.321 e. $\AA^{-3}$} \\
\hline
\end{tabular}


Table S-2. Atomic coordinates (x 104) and equivalent isotropic displacement parameters $\left(\AA^{2} \times 10^{3}\right)$ for $\mathrm{L}^{\mathrm{Me}} \mathrm{Fe}\left(\mathrm{CPh}_{3}\right](\mathbf{1}) . \mathrm{U}_{\text {eq }}$ is defined as one third of the trace of the orthogonalized $\mathrm{U}_{\mathrm{ij}}$ tensor.

\begin{tabular}{|c|c|c|c|c|}
\hline & $\mathrm{x}$ & $\mathrm{y}$ & z & $\mathrm{U}_{\mathrm{eq}}$ \\
\hline $\mathrm{Fe} 1$ & $7166(1)$ & $2398(1)$ & $4172(1)$ & $19(1)$ \\
\hline N21 & $6398(1)$ & $3723(2)$ & $4137(1)$ & $23(1)$ \\
\hline N11 & $7948(1)$ & $3743(2)$ & $4440(1)$ & $22(1)$ \\
\hline $\mathrm{C} 31$ & $7163(2)$ & $5628(3)$ & $4207(1)$ & $25(1)$ \\
\hline C114 & $8035(2)$ & $-318(3)$ & $2473(2)$ & $30(1)$ \\
\hline $\mathrm{C} 74$ & $8350(2)$ & $-7(3)$ & $4875(1)$ & $26(1)$ \\
\hline $\mathrm{C} 34$ & $7327(2)$ & $-1058(3)$ & 4989(1) & $30(1)$ \\
\hline C94 & $8009(2)$ & $-899(3)$ & $3496(1)$ & $25(1)$ \\
\hline $\mathrm{C} 22$ & $8821(2)$ & $3137(3)$ & $5428(1)$ & $28(1)$ \\
\hline C144 & $6446(2)$ & $-180(3)$ & $3756(1)$ & $24(1)$ \\
\hline $\mathrm{C} 12$ & $8651(2)$ & $3288(3)$ & $4779(1)$ & $23(1)$ \\
\hline C62 & $9141(2)$ & 2976(3) & $4471(1)$ & $24(1)$ \\
\hline $\mathrm{C} 13$ & $5706(2)$ & $3311(3)$ & $4140(2)$ & $26(1)$ \\
\hline C63 & $5620(2)$ & 2922(3) & $4711(2)$ & $30(1)$ \\
\hline $\mathrm{C} 23$ & $5139(2)$ & $3291(3)$ & $3586(2)$ & $33(1)$ \\
\hline C134 & $7357(2)$ & $975(3)$ & 2974(1) & $26(1)$ \\
\hline $\mathrm{C} 24$ & $7623(2)$ & $-258(3)$ & $4627(1)$ & $23(1)$ \\
\hline $\mathrm{C} 41$ & $6507(2)$ & $5040(3)$ & $4139(1)$ & $25(1)$ \\
\hline $\mathrm{C} 21$ & $7836(2)$ & $5051(3)$ & $4397(1)$ & $23(1)$ \\
\hline $\mathrm{C} 14$ & $7200(2)$ & $328(3)$ & $4000(1)$ & $23(1)$ \\
\hline $\mathrm{C} 42$ & $9968(2)$ & $2308(3)$ & $5457(2)$ & $35(1)$ \\
\hline $\mathrm{C} 154$ & $6189(2)$ & $-939(3)$ & $3218(1)$ & $30(1)$ \\
\hline $\mathrm{C} 174$ & $5022(2)$ & $-1013(4)$ & $3313(2)$ & $37(1)$ \\
\hline C64 & $8752(2)$ & $-508(3)$ & $5446(2)$ & $32(1)$ \\
\hline C84 & $7553(2)$ & 153(3) & $3498(1)$ & $22(1)$ \\
\hline $\mathrm{C} 32$ & $9484(2)$ & $2645(3)$ & $5757(2)$ & $33(1)$ \\
\hline C112 & $9196(2)$ & 1992(3) & $3461(2)$ & $40(1)$ \\
\hline $\mathrm{C} 103$ & $6242(2)$ & $2905(3)$ & $5308(2)$ & $34(1)$ \\
\hline C54 & $8445(2)$ & $-1285(3)$ & $5797(2)$ & $37(1)$ \\
\hline C104 & $8249(2)$ & $-1122(3)$ & 2993(2) & $29(1)$ \\
\hline $\mathrm{C} 102$ & $8984(2)$ & 3180(3) & $3774(2)$ & $31(1)$ \\
\hline $\mathrm{C} 124$ & $7593(2)$ & $742(3)$ & $2466(1)$ & $28(1)$ \\
\hline
\end{tabular}




\begin{tabular}{|c|c|c|c|c|}
\hline $\mathrm{C} 43$ & $4387(2)$ & 2535(4) & $4175(2)$ & $46(1)$ \\
\hline $\mathrm{C} 184$ & $5272(2)$ & $-283(3)$ & $3860(2)$ & $33(1)$ \\
\hline C51 & $5905(2)$ & 5985(3) & 4091(2) & $36(1)$ \\
\hline $\mathrm{C} 11$ & $8445(2)$ & $6006(3)$ & $4584(2)$ & $39(1)$ \\
\hline $\mathrm{C} 82$ & $8176(2)$ & 2379(4) & $6158(2)$ & $44(1)$ \\
\hline $\mathrm{C} 72$ & $8308(2)$ & $3522(3)$ & $5775(2)$ & $34(1)$ \\
\hline $\mathrm{C} 33$ & $4476(2)$ & 2899(3) & $3617(2)$ & $44(1)$ \\
\hline C194 & $5961(2)$ & $128(3)$ & $4071(2)$ & $27(1)$ \\
\hline $\mathrm{C} 44$ & $7731(2)$ & $-1558(3)$ & $5562(2)$ & $36(1)$ \\
\hline $\mathrm{C} 164$ & 5492(2) & $-1349(4)$ & 2999(2) & $37(1)$ \\
\hline $\mathrm{C} 83$ & $5270(2)$ & $2317(4)$ & $2613(2)$ & $46(1)$ \\
\hline $\mathrm{C} 122$ & $9352(3)$ & 4411(4) & $3635(2)$ & $65(1)$ \\
\hline $\mathrm{C} 73$ & $5236(2)$ & $3604(4)$ & 2961(2) & $42(1)$ \\
\hline $\mathrm{C} 53$ & $4950(2)$ & $2546(3)$ & $4711(2)$ & $38(1)$ \\
\hline C52 & $9797(2)$ & 2489(3) & $4823(2)$ & $30(1)$ \\
\hline C93 & $4668(2)$ & $4543(4)$ & $2556(2)$ & $63(1)$ \\
\hline $\mathrm{C} 123$ & $6199(2)$ & 1792(4) & $5749(2)$ & $54(1)$ \\
\hline C113 & $6353(2)$ & 4242(4) & $5631(2)$ & $59(1)$ \\
\hline C92 & $8577(2)$ & $4740(4)$ & $6186(2)$ & $65(1)$ \\
\hline $\mathrm{C} 15$ & $7319(8)$ & $4170(20)$ & $7697(7)$ & $168(8)$ \\
\hline $\mathrm{C} 25$ & $7948(6)$ & $4984(10)$ & $7654(4)$ & $76(3)$ \\
\hline O15 & 7587(4) & $6180(9)$ & $7405(4)$ & $118(3)$ \\
\hline $\mathrm{C} 35$ & $8036(5)$ & 7321(9) & $7402(4)$ & $63(3)$ \\
\hline $\mathrm{C} 45$ & $7478(8)$ & $8393(16)$ & $7215(7)$ & $168(8)$ \\
\hline
\end{tabular}


Table S-3. Bond lengths $[\AA]$ and angles $\left[^{\circ}\right]$ for $\mathrm{L}^{\mathrm{Me}} \mathrm{Fe}\left(\mathrm{CPh}_{3}\right]$ (1).

\begin{tabular}{|c|c|c|c|}
\hline $\mathrm{Fe}(1)-\mathrm{N}(11)$ & $2.008(2)$ & $\mathrm{C}(23)-\mathrm{C}(33)$ & $1.403(5)$ \\
\hline $\mathrm{Fe}(1)-\mathrm{N}(21)$ & $2.013(2)$ & $\mathrm{C}(23)-\mathrm{C}(73)$ & $1.520(5)$ \\
\hline $\mathrm{Fe}(1)-\mathrm{C}(14)$ & $2.123(3)$ & $\mathrm{C}(134)-\mathrm{C}(124)$ & $1.392(4)$ \\
\hline $\mathrm{N}(21)-\mathrm{C}(41)$ & $1.342(4)$ & $\mathrm{C}(134)-\mathrm{C}(84)$ & $1.396(4)$ \\
\hline $\mathrm{N}(21)-\mathrm{C}(13)$ & $1.444(4)$ & $\mathrm{C}(134)-\mathrm{H}(134)$ & 0.9500 \\
\hline $\mathrm{N}(11)-\mathrm{C}(21)$ & $1.333(4)$ & $C(24)-C(14)$ & $1.524(4)$ \\
\hline $\mathrm{N}(11)-\mathrm{C}(12)$ & $1.444(4)$ & $\mathrm{C}(41)-\mathrm{C}(51)$ & $1.508(4)$ \\
\hline$C(31)-C(21)$ & $1.399(4)$ & $\mathrm{C}(21)-\mathrm{C}(11)$ & $1.501(4)$ \\
\hline $\mathrm{C}(31)-\mathrm{C}(41)$ & $1.400(4)$ & $\mathrm{C}(14)-\mathrm{C}(84)$ & $1.525(4)$ \\
\hline $\mathrm{C}(31)-\mathrm{H}(31)$ & 0.9500 & $\mathrm{C}(42)-\mathrm{C}(52)$ & $1.378(5)$ \\
\hline $\mathrm{C}(114)-\mathrm{C}(124)$ & $1.380(5)$ & $\mathrm{C}(42)-\mathrm{C}(32)$ & $1.387(5)$ \\
\hline$C(114)-C(104)$ & $1.380(4)$ & $\mathrm{C}(42)-\mathrm{H}(42)$ & 0.9500 \\
\hline $\mathrm{C}(114)-\mathrm{H}(114)$ & 0.9500 & $\mathrm{C}(154)-\mathrm{C}(164)$ & $1.383(4)$ \\
\hline$C(74)-C(64)$ & $1.383(4)$ & $\mathrm{C}(154)-\mathrm{H}(154)$ & 0.9500 \\
\hline$C(74)-C(24)$ & $1.403(4)$ & $\mathrm{C}(174)-\mathrm{C}(164)$ & $1.387(5)$ \\
\hline $\mathrm{C}(74)-\mathrm{H}(74)$ & 0.9500 & $\mathrm{C}(174)-\mathrm{C}(184)$ & $1.389(5)$ \\
\hline$C(34)-C(44)$ & $1.386(4)$ & $\mathrm{C}(174)-\mathrm{H}(174)$ & 0.9500 \\
\hline$C(34)-C(24)$ & $1.405(4)$ & $\mathrm{C}(64)-\mathrm{C}(54)$ & $1.387(5)$ \\
\hline $\mathrm{C}(34)-\mathrm{H}(34)$ & 0.9500 & $\mathrm{C}(64)-\mathrm{H}(64)$ & 0.9500 \\
\hline C(94)-C(104) & $1.386(4)$ & $\mathrm{C}(32)-\mathrm{H}(32)$ & 0.9500 \\
\hline $\mathrm{C}(94)-\mathrm{C}(84)$ & $1.396(4)$ & $\mathrm{C}(112)-\mathrm{C}(102)$ & $1.517(5)$ \\
\hline $\mathrm{C}(94)-\mathrm{H}(94)$ & 0.9500 & $\mathrm{C}(112)-\mathrm{H}(11 \mathrm{~A})$ & 0.9800 \\
\hline$C(22)-C(32)$ & $1.388(4)$ & $\mathrm{C}(112)-\mathrm{H}(11 \mathrm{~B})$ & 0.9800 \\
\hline$C(22)-C(12)$ & $1.407(4)$ & $\mathrm{C}(112)-\mathrm{H}(11 \mathrm{C})$ & 0.9800 \\
\hline$C(22)-C(72)$ & $1.525(4)$ & $\mathrm{C}(103)-\mathrm{C}(113)$ & $1.513(5)$ \\
\hline$C(144)-C(154)$ & $1.391(4)$ & $\mathrm{C}(103)-\mathrm{C}(123)$ & $1.519(5)$ \\
\hline$C(144)-C(194)$ & $1.405(4)$ & $\mathrm{C}(103)-\mathrm{H}(103)$ & 1.0000 \\
\hline$C(144)-C(14)$ & $1.517(4)$ & $\mathrm{C}(54)-\mathrm{C}(44)$ & $1.382(5)$ \\
\hline$C(12)-C(62)$ & $1.403(4)$ & $\mathrm{C}(54)-\mathrm{H}(54)$ & 0.9500 \\
\hline$C(62)-C(52)$ & $1.389(4)$ & $\mathrm{C}(104)-\mathrm{H}(104)$ & 0.9500 \\
\hline$C(62)-C(102)$ & $1.522(4)$ & $\mathrm{C}(102)-\mathrm{C}(122)$ & $1.521(5)$ \\
\hline$C(13)-C(23)$ & $1.397(4)$ & $\mathrm{C}(102)-\mathrm{H}(102)$ & 1.0000 \\
\hline$C(13)-C(63)$ & $1.412(4)$ & $\mathrm{C}(124)-\mathrm{H}(124)$ & 0.9500 \\
\hline$C(63)-C(53)$ & $1.390(4)$ & $\mathrm{C}(43)-\mathrm{C}(53)$ & $1.368(5)$ \\
\hline$C(63)-C(103)$ & $1.519(5)$ & $\mathrm{C}(43)-\mathrm{C}(33)$ & $1.380(5)$ \\
\hline
\end{tabular}




\begin{tabular}{|c|c|c|c|}
\hline $\mathrm{C}(43)-\mathrm{H}(43)$ & 0.9500 & $\mathrm{C}(113)-\mathrm{H}(11 \mathrm{H})$ & 0.9800 \\
\hline C(184)-C(194) & $1.368(4)$ & $\mathrm{C}(113)-\mathrm{H}(11 \mathrm{I})$ & 0.9800 \\
\hline C(184)-H(184) & 0.9500 & $\mathrm{C}(92)-\mathrm{H}(92 \mathrm{~A})$ & 0.9800 \\
\hline $\mathrm{C}(51)-\mathrm{H}(51 \mathrm{~A})$ & 0.9800 & $\mathrm{C}(92)-\mathrm{H}(92 \mathrm{~B})$ & 0.9800 \\
\hline $\mathrm{C}(51)-\mathrm{H}(51 \mathrm{~B})$ & 0.9800 & $\mathrm{C}(92)-\mathrm{H}(92 \mathrm{C})$ & 0.9800 \\
\hline $\mathrm{C}(51)-\mathrm{H}(51 \mathrm{C})$ & 0.9800 & $C(15)-C(25)$ & $1.524(9)$ \\
\hline $\mathrm{C}(11)-\mathrm{H}(11 \mathrm{D})$ & 0.9800 & $\mathrm{C}(15)-\mathrm{H}(15 \mathrm{~A})$ & 0.9800 \\
\hline $\mathrm{C}(11)-\mathrm{H}(11 \mathrm{E})$ & 0.9800 & $\mathrm{C}(15)-\mathrm{H}(15 \mathrm{~B})$ & 0.9800 \\
\hline $\mathrm{C}(11)-\mathrm{H}(11 \mathrm{~F})$ & 0.9800 & $\mathrm{C}(15)-\mathrm{H}(15 \mathrm{C})$ & 0.9800 \\
\hline$C(82)-C(72)$ & $1.511(5)$ & $\mathrm{C}(25)-\mathrm{O}(15)$ & $1.423(10)$ \\
\hline $\mathrm{C}(82)-\mathrm{H}(82 \mathrm{~A})$ & 0.9800 & $\mathrm{C}(25)-\mathrm{H}(25 \mathrm{~A})$ & 0.9900 \\
\hline $\mathrm{C}(82)-\mathrm{H}(82 \mathrm{~B})$ & 0.9800 & $\mathrm{C}(25)-\mathrm{H}(25 \mathrm{~B})$ & 0.9900 \\
\hline $\mathrm{C}(82)-\mathrm{H}(82 \mathrm{C})$ & 0.9800 & $\mathrm{O}(15)-\mathrm{C}(35)$ & $1.457(10)$ \\
\hline$C(72)-C(92)$ & $1.530(5)$ & $C(35)-C(45)$ & $1.512(9)$ \\
\hline $\mathrm{C}(72)-\mathrm{H}(72)$ & 1.0000 & $\mathrm{C}(35)-\mathrm{H}(35 \mathrm{~A})$ & 0.9900 \\
\hline $\mathrm{C}(33)-\mathrm{H}(33)$ & 0.9500 & $\mathrm{C}(35)-\mathrm{H}(35 \mathrm{~B})$ & 0.9900 \\
\hline C(194)-H(194) & 0.9500 & $\mathrm{C}(45)-\mathrm{H}(45 \mathrm{~A})$ & 0.9800 \\
\hline $\mathrm{C}(44)-\mathrm{H}(44)$ & 0.9500 & $\mathrm{C}(45)-\mathrm{H}(45 \mathrm{~B})$ & 0.9800 \\
\hline $\mathrm{C}(164)-\mathrm{H}(164)$ & 0.9500 & $\mathrm{C}(45)-\mathrm{H}(45 \mathrm{C})$ & 0.9800 \\
\hline$C(83)-C(73)$ & $1.528(5)$ & $\mathrm{N}(11)-\mathrm{Fe}(1)-\mathrm{N}(21)$ & $94.29(10)$ \\
\hline $\mathrm{C}(83)-\mathrm{H}(83 \mathrm{~A})$ & 0.9800 & $\mathrm{~N}(11)-\mathrm{Fe}(1)-\mathrm{C}(14)$ & $130.49(11)$ \\
\hline $\mathrm{C}(83)-\mathrm{H}(83 \mathrm{~B})$ & 0.9800 & $\mathrm{~N}(21)-\mathrm{Fe}(1)-\mathrm{C}(14)$ & $135.23(11)$ \\
\hline $\mathrm{C}(83)-\mathrm{H}(83 \mathrm{C})$ & 0.9800 & $\mathrm{C}(41)-\mathrm{N}(21)-\mathrm{C}(13)$ & $116.0(2)$ \\
\hline $\mathrm{C}(122)-\mathrm{H}(12 \mathrm{~A})$ & 0.9800 & $\mathrm{C}(41)-\mathrm{N}(21)-\mathrm{Fe}(1)$ & $122.2(2)$ \\
\hline $\mathrm{C}(122)-\mathrm{H}(12 \mathrm{~B})$ & 0.9800 & $\mathrm{C}(13)-\mathrm{N}(21)-\mathrm{Fe}(1)$ & $121.77(19)$ \\
\hline $\mathrm{C}(122)-\mathrm{H}(12 \mathrm{C})$ & 0.9800 & $\mathrm{C}(21)-\mathrm{N}(11)-\mathrm{C}(12)$ & $117.7(2)$ \\
\hline$C(73)-C(93)$ & $1.537(5)$ & $\mathrm{C}(21)-\mathrm{N}(11)-\mathrm{Fe}(1)$ & $123.1(2)$ \\
\hline $\mathrm{C}(73)-\mathrm{H}(73)$ & 1.0000 & $\mathrm{C}(12)-\mathrm{N}(11)-\mathrm{Fe}(1)$ & $118.58(18)$ \\
\hline $\mathrm{C}(53)-\mathrm{H}(53)$ & 0.9500 & $\mathrm{C}(21)-\mathrm{C}(31)-\mathrm{C}(41)$ & 129.1(3) \\
\hline $\mathrm{C}(52)-\mathrm{H}(52)$ & 0.9500 & $\mathrm{C}(21)-\mathrm{C}(31)-\mathrm{H}(31)$ & 115.4 \\
\hline $\mathrm{C}(93)-\mathrm{H}(93 \mathrm{~A})$ & 0.9800 & $\mathrm{C}(41)-\mathrm{C}(31)-\mathrm{H}(31)$ & 115.4 \\
\hline C(93)-H(93B) & 0.9800 & $\mathrm{C}(124)-\mathrm{C}(114)-\mathrm{C}(104)$ & 119.2(3) \\
\hline $\mathrm{C}(93)-\mathrm{H}(93 \mathrm{C})$ & 0.9800 & $\mathrm{C}(124)-\mathrm{C}(114)-\mathrm{H}(114)$ & 120.4 \\
\hline $\mathrm{C}(123)-\mathrm{H}(12 \mathrm{D})$ & 0.9800 & $\mathrm{C}(104)-\mathrm{C}(114)-\mathrm{H}(114)$ & 120.4 \\
\hline $\mathrm{C}(123)-\mathrm{H}(12 \mathrm{E})$ & 0.9800 & $C(64)-C(74)-C(24)$ & $122.0(3)$ \\
\hline $\mathrm{C}(123)-\mathrm{H}(12 \mathrm{~F})$ & 0.9800 & $\mathrm{C}(64)-\mathrm{C}(74)-\mathrm{H}(74)$ & 119.0 \\
\hline $\mathrm{C}(113)-\mathrm{H}(11 \mathrm{G})$ & 0.9800 & $\mathrm{C}(24)-\mathrm{C}(74)-\mathrm{H}(74)$ & 119.0 \\
\hline
\end{tabular}




\begin{tabular}{|c|c|c|c|}
\hline $\mathrm{C}(44)-\mathrm{C}(34)-\mathrm{C}(24)$ & $121.8(3)$ & $\mathrm{N}(11)-\mathrm{C}(21)-\mathrm{C}(11)$ & $120.5(3)$ \\
\hline $\mathrm{C}(44)-\mathrm{C}(34)-\mathrm{H}(34)$ & 119.1 & $\mathrm{C}(31)-\mathrm{C}(21)-\mathrm{C}(11)$ & $115.7(3)$ \\
\hline $\mathrm{C}(24)-\mathrm{C}(34)-\mathrm{H}(34)$ & 119.1 & $C(144)-C(14)-C(24)$ & $113.7(2)$ \\
\hline $\mathrm{C}(104)-\mathrm{C}(94)-\mathrm{C}(84)$ & $121.3(3)$ & $\mathrm{C}(144)-\mathrm{C}(14)-\mathrm{C}(84)$ & 109.2(2) \\
\hline C(104)-C(94)-H(94) & 119.4 & $C(24)-C(14)-C(84)$ & $113.6(2)$ \\
\hline $\mathrm{C}(84)-\mathrm{C}(94)-\mathrm{H}(94)$ & 119.4 & $\mathrm{C}(144)-\mathrm{C}(14)-\mathrm{Fe}(1)$ & $108.19(19)$ \\
\hline$C(32)-C(22)-C(12)$ & $118.2(3)$ & $\mathrm{C}(24)-\mathrm{C}(14)-\mathrm{Fe}(1)$ & $104.37(19)$ \\
\hline $\mathrm{C}(32)-\mathrm{C}(22)-\mathrm{C}(72)$ & $119.6(3)$ & $\mathrm{C}(84)-\mathrm{C}(14)-\mathrm{Fe}(1)$ & $107.41(19)$ \\
\hline $\mathrm{C}(12)-\mathrm{C}(22)-\mathrm{C}(72)$ & $122.1(3)$ & $\mathrm{C}(52)-\mathrm{C}(42)-\mathrm{C}(32)$ & 119.7(3) \\
\hline C(154)-C(144)-C(194) & $116.3(3)$ & $\mathrm{C}(52)-\mathrm{C}(42)-\mathrm{H}(42)$ & 120.1 \\
\hline $\mathrm{C}(154)-\mathrm{C}(144)-\mathrm{C}(14)$ & $123.5(3)$ & $\mathrm{C}(32)-\mathrm{C}(42)-\mathrm{H}(42)$ & 120.1 \\
\hline C(194)-C(144)-C(14) & $120.2(3)$ & $C(164)-C(154)-C(144)$ & $121.9(3)$ \\
\hline$C(62)-C(12)-C(22)$ & $121.2(3)$ & $\mathrm{C}(164)-\mathrm{C}(154)-\mathrm{H}(154)$ & 119.1 \\
\hline $\mathrm{C}(62)-\mathrm{C}(12)-\mathrm{N}(11)$ & $121.2(3)$ & $\mathrm{C}(144)-\mathrm{C}(154)-\mathrm{H}(154)$ & 119.1 \\
\hline $\mathrm{C}(22)-\mathrm{C}(12)-\mathrm{N}(11)$ & $117.6(3)$ & $\mathrm{C}(164)-\mathrm{C}(174)-\mathrm{C}(184)$ & $118.3(3)$ \\
\hline$C(52)-C(62)-C(12)$ & $118.2(3)$ & $\mathrm{C}(164)-\mathrm{C}(174)-\mathrm{H}(174)$ & 120.8 \\
\hline $\mathrm{C}(52)-\mathrm{C}(62)-\mathrm{C}(102)$ & $119.3(3)$ & $\mathrm{C}(184)-\mathrm{C}(174)-\mathrm{H}(174)$ & 120.8 \\
\hline$C(12)-C(62)-C(102)$ & $122.5(3)$ & $\mathrm{C}(74)-\mathrm{C}(64)-\mathrm{C}(54)$ & $120.7(3)$ \\
\hline$C(23)-C(13)-C(63)$ & $121.3(3)$ & $\mathrm{C}(74)-\mathrm{C}(64)-\mathrm{H}(64)$ & 119.6 \\
\hline $\mathrm{C}(23)-\mathrm{C}(13)-\mathrm{N}(21)$ & $120.3(3)$ & $\mathrm{C}(54)-\mathrm{C}(64)-\mathrm{H}(64)$ & 119.6 \\
\hline $\mathrm{C}(63)-\mathrm{C}(13)-\mathrm{N}(21)$ & $118.4(3)$ & $\mathrm{C}(134)-\mathrm{C}(84)-\mathrm{C}(94)$ & $117.0(3)$ \\
\hline$C(53)-C(63)-C(13)$ & $118.0(3)$ & $\mathrm{C}(134)-\mathrm{C}(84)-\mathrm{C}(14)$ & $119.6(3)$ \\
\hline $\mathrm{C}(53)-\mathrm{C}(63)-\mathrm{C}(103)$ & $120.8(3)$ & $\mathrm{C}(94)-\mathrm{C}(84)-\mathrm{C}(14)$ & $123.0(3)$ \\
\hline$C(13)-C(63)-C(103)$ & $121.2(3)$ & $C(42)-C(32)-C(22)$ & $121.2(3)$ \\
\hline$C(13)-C(23)-C(33)$ & $117.9(3)$ & $\mathrm{C}(42)-\mathrm{C}(32)-\mathrm{H}(32)$ & 119.4 \\
\hline$C(13)-C(23)-C(73)$ & $121.8(3)$ & $\mathrm{C}(22)-\mathrm{C}(32)-\mathrm{H}(32)$ & 119.4 \\
\hline $\mathrm{C}(33)-\mathrm{C}(23)-\mathrm{C}(73)$ & $120.1(3)$ & $\mathrm{C}(102)-\mathrm{C}(112)-\mathrm{H}(11 \mathrm{~A})$ & 109.5 \\
\hline $\mathrm{C}(124)-\mathrm{C}(134)-\mathrm{C}(84)$ & $121.6(3)$ & $\mathrm{C}(102)-\mathrm{C}(112)-\mathrm{H}(11 \mathrm{~B})$ & 109.5 \\
\hline C(124)-C(134)-H(134) & 119.2 & $\mathrm{H}(11 \mathrm{~A})-\mathrm{C}(112)-\mathrm{H}(11 \mathrm{~B})$ & 109.5 \\
\hline C(84)-C(134)-H(134) & 119.2 & $\mathrm{C}(102)-\mathrm{C}(112)-\mathrm{H}(11 \mathrm{C})$ & 109.5 \\
\hline$C(74)-C(24)-C(34)$ & $116.1(3)$ & $\mathrm{H}(11 \mathrm{~A})-\mathrm{C}(112)-\mathrm{H}(11 \mathrm{C})$ & 109.5 \\
\hline $\mathrm{C}(74)-\mathrm{C}(24)-\mathrm{C}(14)$ & $120.2(3)$ & $\mathrm{H}(11 \mathrm{~B})-\mathrm{C}(112)-\mathrm{H}(11 \mathrm{C})$ & 109.5 \\
\hline $\mathrm{C}(34)-\mathrm{C}(24)-\mathrm{C}(14)$ & $123.7(3)$ & $\mathrm{C}(113)-\mathrm{C}(103)-\mathrm{C}(123)$ & $111.6(3)$ \\
\hline $\mathrm{N}(21)-\mathrm{C}(41)-\mathrm{C}(31)$ & $124.2(3)$ & $\mathrm{C}(113)-\mathrm{C}(103)-\mathrm{C}(63)$ & $111.9(3)$ \\
\hline $\mathrm{N}(21)-\mathrm{C}(41)-\mathrm{C}(51)$ & $119.8(3)$ & $\mathrm{C}(123)-\mathrm{C}(103)-\mathrm{C}(63)$ & 113.2(3) \\
\hline $\mathrm{C}(31)-\mathrm{C}(41)-\mathrm{C}(51)$ & $115.9(3)$ & $\mathrm{C}(113)-\mathrm{C}(103)-\mathrm{H}(103)$ & 106.5 \\
\hline $\mathrm{N}(11)-\mathrm{C}(21)-\mathrm{C}(31)$ & $123.7(3)$ & $\mathrm{C}(123)-\mathrm{C}(103)-\mathrm{H}(103)$ & 106.5 \\
\hline
\end{tabular}




\begin{tabular}{|c|c|c|c|}
\hline C(63)-C(103)-H(103) & 106.5 & $\mathrm{C}(72)-\mathrm{C}(82)-\mathrm{H}(82 \mathrm{C})$ & 109.5 \\
\hline $\mathrm{C}(44)-\mathrm{C}(54)-\mathrm{C}(64)$ & $118.6(3)$ & $\mathrm{H}(82 \mathrm{~A})-\mathrm{C}(82)-\mathrm{H}(82 \mathrm{C})$ & 109.5 \\
\hline $\mathrm{C}(44)-\mathrm{C}(54)-\mathrm{H}(54)$ & 120.7 & $\mathrm{H}(82 \mathrm{~B})-\mathrm{C}(82)-\mathrm{H}(82 \mathrm{C})$ & 109.5 \\
\hline $\mathrm{C}(64)-\mathrm{C}(54)-\mathrm{H}(54)$ & 120.7 & $\mathrm{C}(82)-\mathrm{C}(72)-\mathrm{C}(22)$ & $111.7(3)$ \\
\hline $\mathrm{C}(114)-\mathrm{C}(104)-\mathrm{C}(94)$ & $120.8(3)$ & $\mathrm{C}(82)-\mathrm{C}(72)-\mathrm{C}(92)$ & $110.6(3)$ \\
\hline C(114)-C(104)-H(104) & 119.6 & $\mathrm{C}(22)-\mathrm{C}(72)-\mathrm{C}(92)$ & $110.6(3)$ \\
\hline C(94)-C(104)-H(104) & 119.6 & $\mathrm{C}(82)-\mathrm{C}(72)-\mathrm{H}(72)$ & 107.9 \\
\hline$C(112)-C(102)-C(122)$ & $108.7(3)$ & $\mathrm{C}(22)-\mathrm{C}(72)-\mathrm{H}(72)$ & 107.9 \\
\hline $\mathrm{C}(112)-\mathrm{C}(102)-\mathrm{C}(62)$ & $112.4(3)$ & $\mathrm{C}(92)-\mathrm{C}(72)-\mathrm{H}(72)$ & 107.9 \\
\hline$C(122)-C(102)-C(62)$ & $111.7(3)$ & $C(43)-C(33)-C(23)$ & $121.0(3)$ \\
\hline $\mathrm{C}(112)-\mathrm{C}(102)-\mathrm{H}(102)$ & 107.9 & $\mathrm{C}(43)-\mathrm{C}(33)-\mathrm{H}(33)$ & 119.5 \\
\hline $\mathrm{C}(122)-\mathrm{C}(102)-\mathrm{H}(102)$ & 107.9 & $\mathrm{C}(23)-\mathrm{C}(33)-\mathrm{H}(33)$ & 119.5 \\
\hline $\mathrm{C}(62)-\mathrm{C}(102)-\mathrm{H}(102)$ & 107.9 & C(184)-C(194)-C(144) & $122.1(3)$ \\
\hline C(114)-C(124)-C(134) & $120.1(3)$ & C(184)-C(194)-H(194) & 118.9 \\
\hline $\mathrm{C}(114)-\mathrm{C}(124)-\mathrm{H}(124)$ & 119.9 & $\mathrm{C}(144)-\mathrm{C}(194)-\mathrm{H}(194)$ & 118.9 \\
\hline C(134)-C(124)-H(124) & 119.9 & $\mathrm{C}(54)-\mathrm{C}(44)-\mathrm{C}(34)$ & $120.8(3)$ \\
\hline $\mathrm{C}(53)-\mathrm{C}(43)-\mathrm{C}(33)$ & $120.3(3)$ & $\mathrm{C}(54)-\mathrm{C}(44)-\mathrm{H}(44)$ & 119.6 \\
\hline $\mathrm{C}(53)-\mathrm{C}(43)-\mathrm{H}(43)$ & 119.9 & $\mathrm{C}(34)-\mathrm{C}(44)-\mathrm{H}(44)$ & 119.6 \\
\hline $\mathrm{C}(33)-\mathrm{C}(43)-\mathrm{H}(43)$ & 119.9 & C(154)-C(164)-C(174) & $120.7(3)$ \\
\hline C(194)-C(184)-C(174) & $120.7(3)$ & $\mathrm{C}(154)-\mathrm{C}(164)-\mathrm{H}(164)$ & 119.7 \\
\hline C(194)-C(184)-H(184) & 119.7 & $\mathrm{C}(174)-\mathrm{C}(164)-\mathrm{H}(164)$ & 119.7 \\
\hline C(174)-C(184)-H(184) & 119.7 & $\mathrm{C}(73)-\mathrm{C}(83)-\mathrm{H}(83 \mathrm{~A})$ & 109.5 \\
\hline $\mathrm{C}(41)-\mathrm{C}(51)-\mathrm{H}(51 \mathrm{~A})$ & 109.5 & $\mathrm{C}(73)-\mathrm{C}(83)-\mathrm{H}(83 \mathrm{~B})$ & 109.5 \\
\hline $\mathrm{C}(41)-\mathrm{C}(51)-\mathrm{H}(51 \mathrm{~B})$ & 109.5 & $\mathrm{H}(83 \mathrm{~A})-\mathrm{C}(83)-\mathrm{H}(83 \mathrm{~B})$ & 109.5 \\
\hline $\mathrm{H}(51 \mathrm{~A})-\mathrm{C}(51)-\mathrm{H}(51 \mathrm{~B})$ & 109.5 & $\mathrm{C}(73)-\mathrm{C}(83)-\mathrm{H}(83 \mathrm{C})$ & 109.5 \\
\hline $\mathrm{C}(41)-\mathrm{C}(51)-\mathrm{H}(51 \mathrm{C})$ & 109.5 & $\mathrm{H}(83 \mathrm{~A})-\mathrm{C}(83)-\mathrm{H}(83 \mathrm{C})$ & 109.5 \\
\hline $\mathrm{H}(51 \mathrm{~A})-\mathrm{C}(51)-\mathrm{H}(51 \mathrm{C})$ & 109.5 & $\mathrm{H}(83 \mathrm{~B})-\mathrm{C}(83)-\mathrm{H}(83 \mathrm{C})$ & 109.5 \\
\hline $\mathrm{H}(51 \mathrm{~B})-\mathrm{C}(51)-\mathrm{H}(51 \mathrm{C})$ & 109.5 & $\mathrm{C}(102)-\mathrm{C}(122)-\mathrm{H}(12 \mathrm{~A})$ & 109.5 \\
\hline $\mathrm{C}(21)-\mathrm{C}(11)-\mathrm{H}(11 \mathrm{D})$ & 109.5 & $\mathrm{C}(102)-\mathrm{C}(122)-\mathrm{H}(12 \mathrm{~B})$ & 109.5 \\
\hline $\mathrm{C}(21)-\mathrm{C}(11)-\mathrm{H}(11 \mathrm{E})$ & 109.5 & $\mathrm{H}(12 \mathrm{~A})-\mathrm{C}(122)-\mathrm{H}(12 \mathrm{~B})$ & 109.5 \\
\hline H(11D)-C(11)-H(11E) & 109.5 & $\mathrm{C}(102)-\mathrm{C}(122)-\mathrm{H}(12 \mathrm{C})$ & 109.5 \\
\hline $\mathrm{C}(21)-\mathrm{C}(11)-\mathrm{H}(11 \mathrm{~F})$ & 109.5 & $\mathrm{H}(12 \mathrm{~A})-\mathrm{C}(122)-\mathrm{H}(12 \mathrm{C})$ & 109.5 \\
\hline H(11D)-C(11)-H(11F) & 109.5 & $\mathrm{H}(12 \mathrm{~B})-\mathrm{C}(122)-\mathrm{H}(12 \mathrm{C})$ & 109.5 \\
\hline $\mathrm{H}(11 \mathrm{E})-\mathrm{C}(11)-\mathrm{H}(11 \mathrm{~F})$ & 109.5 & $\mathrm{C}(23)-\mathrm{C}(73)-\mathrm{C}(83)$ & $110.1(3)$ \\
\hline $\mathrm{C}(72)-\mathrm{C}(82)-\mathrm{H}(82 \mathrm{~A})$ & 109.5 & $\mathrm{C}(23)-\mathrm{C}(73)-\mathrm{C}(93)$ & $113.4(3)$ \\
\hline $\mathrm{C}(72)-\mathrm{C}(82)-\mathrm{H}(82 \mathrm{~B})$ & 109.5 & $\mathrm{C}(83)-\mathrm{C}(73)-\mathrm{C}(93)$ & $110.9(3)$ \\
\hline H(82A)-C(82)-H(82B) & 109.5 & $\mathrm{C}(23)-\mathrm{C}(73)-\mathrm{H}(73)$ & 107.4 \\
\hline
\end{tabular}




\begin{tabular}{|c|c|c|c|}
\hline $\mathrm{C}(83)-\mathrm{C}(73)-\mathrm{H}(73)$ & 107.4 & $\mathrm{C}(72)-\mathrm{C}(92)-\mathrm{H}(92 \mathrm{C})$ & 109.5 \\
\hline $\mathrm{C}(93)-\mathrm{C}(73)-\mathrm{H}(73)$ & 107.4 & $\mathrm{H}(92 \mathrm{~A})-\mathrm{C}(92)-\mathrm{H}(92 \mathrm{C})$ & 109.5 \\
\hline$C(43)-C(53)-C(63)$ & $121.4(3)$ & $\mathrm{H}(92 \mathrm{~B})-\mathrm{C}(92)-\mathrm{H}(92 \mathrm{C})$ & 109.5 \\
\hline $\mathrm{C}(43)-\mathrm{C}(53)-\mathrm{H}(53)$ & 119.3 & $\mathrm{C}(25)-\mathrm{C}(15)-\mathrm{H}(15 \mathrm{~A})$ & 109.5 \\
\hline $\mathrm{C}(63)-\mathrm{C}(53)-\mathrm{H}(53)$ & 119.3 & $\mathrm{C}(25)-\mathrm{C}(15)-\mathrm{H}(15 \mathrm{~B})$ & 109.5 \\
\hline $\mathrm{C}(42)-\mathrm{C}(52)-\mathrm{C}(62)$ & $121.5(3)$ & $\mathrm{H}(15 \mathrm{~A})-\mathrm{C}(15)-\mathrm{H}(15 \mathrm{~B})$ & 109.5 \\
\hline $\mathrm{C}(42)-\mathrm{C}(52)-\mathrm{H}(52)$ & 119.3 & $\mathrm{C}(25)-\mathrm{C}(15)-\mathrm{H}(15 \mathrm{C})$ & 109.5 \\
\hline $\mathrm{C}(62)-\mathrm{C}(52)-\mathrm{H}(52)$ & 119.3 & $\mathrm{H}(15 \mathrm{~A})-\mathrm{C}(15)-\mathrm{H}(15 \mathrm{C})$ & 109.5 \\
\hline $\mathrm{C}(73)-\mathrm{C}(93)-\mathrm{H}(93 \mathrm{~A})$ & 109.5 & $\mathrm{H}(15 \mathrm{~B})-\mathrm{C}(15)-\mathrm{H}(15 \mathrm{C})$ & 109.5 \\
\hline $\mathrm{C}(73)-\mathrm{C}(93)-\mathrm{H}(93 \mathrm{~B})$ & 109.5 & $\mathrm{O}(15)-\mathrm{C}(25)-\mathrm{C}(15)$ & $98.9(11)$ \\
\hline $\mathrm{H}(93 \mathrm{~A})-\mathrm{C}(93)-\mathrm{H}(93 \mathrm{~B})$ & 109.5 & $\mathrm{O}(15)-\mathrm{C}(25)-\mathrm{H}(25 \mathrm{~A})$ & 112.0 \\
\hline C(73)-C(93)-H(93C) & 109.5 & $\mathrm{C}(15)-\mathrm{C}(25)-\mathrm{H}(25 \mathrm{~A})$ & 112.0 \\
\hline $\mathrm{H}(93 \mathrm{~A})-\mathrm{C}(93)-\mathrm{H}(93 \mathrm{C})$ & 109.5 & $\mathrm{O}(15)-\mathrm{C}(25)-\mathrm{H}(25 \mathrm{~B})$ & 112.0 \\
\hline $\mathrm{H}(93 \mathrm{~B})-\mathrm{C}(93)-\mathrm{H}(93 \mathrm{C})$ & 109.5 & $\mathrm{C}(15)-\mathrm{C}(25)-\mathrm{H}(25 \mathrm{~B})$ & 112.0 \\
\hline $\mathrm{C}(103)-\mathrm{C}(123)-\mathrm{H}(12 \mathrm{D})$ & 109.5 & $\mathrm{H}(25 \mathrm{~A})-\mathrm{C}(25)-\mathrm{H}(25 \mathrm{~B})$ & 109.7 \\
\hline $\mathrm{C}(103)-\mathrm{C}(123)-\mathrm{H}(12 \mathrm{E})$ & 109.5 & $\mathrm{C}(25)-\mathrm{O}(15)-\mathrm{C}(35)$ & $115.7(8)$ \\
\hline $\mathrm{H}(12 \mathrm{D})-\mathrm{C}(123)-\mathrm{H}(12 \mathrm{E})$ & 109.5 & $\mathrm{O}(15)-\mathrm{C}(35)-\mathrm{C}(45)$ & $99.4(10)$ \\
\hline $\mathrm{C}(103)-\mathrm{C}(123)-\mathrm{H}(12 \mathrm{~F})$ & 109.5 & $\mathrm{O}(15)-\mathrm{C}(35)-\mathrm{H}(35 \mathrm{~A})$ & 111.9 \\
\hline $\mathrm{H}(12 \mathrm{D})-\mathrm{C}(123)-\mathrm{H}(12 \mathrm{~F})$ & 109.5 & $\mathrm{C}(45)-\mathrm{C}(35)-\mathrm{H}(35 \mathrm{~A})$ & 111.9 \\
\hline $\mathrm{H}(12 \mathrm{E})-\mathrm{C}(123)-\mathrm{H}(12 \mathrm{~F})$ & 109.5 & $\mathrm{O}(15)-\mathrm{C}(35)-\mathrm{H}(35 \mathrm{~B})$ & 111.9 \\
\hline $\mathrm{C}(103)-\mathrm{C}(113)-\mathrm{H}(11 \mathrm{G})$ & 109.5 & $\mathrm{C}(45)-\mathrm{C}(35)-\mathrm{H}(35 \mathrm{~B})$ & 111.9 \\
\hline $\mathrm{C}(103)-\mathrm{C}(113)-\mathrm{H}(11 \mathrm{H})$ & 109.5 & $\mathrm{H}(35 \mathrm{~A})-\mathrm{C}(35)-\mathrm{H}(35 \mathrm{~B})$ & 109.6 \\
\hline $\mathrm{H}(11 \mathrm{G})-\mathrm{C}(113)-\mathrm{H}(11 \mathrm{H})$ & 109.5 & $\mathrm{C}(35)-\mathrm{C}(45)-\mathrm{H}(45 \mathrm{~A})$ & 109.5 \\
\hline $\mathrm{C}(103)-\mathrm{C}(113)-\mathrm{H}(11 \mathrm{I})$ & 109.5 & $\mathrm{C}(35)-\mathrm{C}(45)-\mathrm{H}(45 \mathrm{~B})$ & 109.5 \\
\hline $\mathrm{H}(11 \mathrm{G})-\mathrm{C}(113)-\mathrm{H}(11 \mathrm{I})$ & 109.5 & $\mathrm{H}(45 \mathrm{~A})-\mathrm{C}(45)-\mathrm{H}(45 \mathrm{~B})$ & 109.5 \\
\hline $\mathrm{H}(11 \mathrm{H})-\mathrm{C}(113)-\mathrm{H}(11 \mathrm{I})$ & 109.5 & $\mathrm{C}(35)-\mathrm{C}(45)-\mathrm{H}(45 \mathrm{C})$ & 109.5 \\
\hline $\mathrm{C}(72)-\mathrm{C}(92)-\mathrm{H}(92 \mathrm{~A})$ & 109.5 & $\mathrm{H}(45 \mathrm{~A})-\mathrm{C}(45)-\mathrm{H}(45 \mathrm{C})$ & 109.5 \\
\hline $\mathrm{C}(72)-\mathrm{C}(92)-\mathrm{H}(92 \mathrm{~B})$ & 109.5 & $\mathrm{H}(45 \mathrm{~B})-\mathrm{C}(45)-\mathrm{H}(45 \mathrm{C})$ & 109.5 \\
\hline $\mathrm{H}(92 \mathrm{~A})-\mathrm{C}(92)-\mathrm{H}(92 \mathrm{~B})$ & 109.5 & & \\
\hline
\end{tabular}


Table S-4. Anisotropic displacement parameters $\left(\AA^{2} \times 10^{3}\right)$ for $\mathrm{L}^{\mathrm{Me}} \mathrm{Fe}\left(\mathrm{CPh}_{3}\right]$ (1). The anisotropic displacement factor exponent takes the form: $-2 \pi^{2}\left[\mathrm{~h}^{2} \mathrm{a}^{* 2} \mathrm{U}_{11}+\ldots+2 \mathrm{~h} \mathrm{k} \mathrm{a} \mathrm{b}^{*} \mathrm{U}_{12}\right]$

\begin{tabular}{|c|c|c|c|c|c|c|}
\hline & $\mathrm{U}_{11}$ & $\mathrm{U}_{22}$ & $\mathrm{U}_{33}$ & $\mathrm{U}_{23}$ & $\mathrm{U}_{13}$ & $\mathrm{U}_{12}$ \\
\hline $\mathrm{Fe} 1$ & $20(1)$ & $16(1)$ & $26(1)$ & $-1(1)$ & $12(1)$ & $0(1)$ \\
\hline N21 & $21(1)$ & 19(1) & $31(1)$ & $-1(1)$ & 11(1) & $-3(1)$ \\
\hline N11 & $22(1)$ & 19(1) & $28(1)$ & $-1(1)$ & $13(1)$ & $2(1)$ \\
\hline C31 & $32(2)$ & $13(2)$ & $33(2)$ & $2(1)$ & $16(1)$ & $0(1)$ \\
\hline C114 & $32(2)$ & $33(2)$ & $30(2)$ & $-7(2)$ & $17(2)$ & $-3(2)$ \\
\hline $\mathrm{C} 74$ & $32(2)$ & $16(2)$ & $32(2)$ & $-3(1)$ & $14(2)$ & $0(1)$ \\
\hline C34 & $36(2)$ & $26(2)$ & $32(2)$ & $0(2)$ & $19(2)$ & $1(2)$ \\
\hline C94 & $31(2)$ & 19(2) & $29(2)$ & $1(1)$ & $14(1)$ & $2(1)$ \\
\hline $\mathrm{C} 22$ & $31(2)$ & $22(2)$ & $30(2)$ & $-2(1)$ & $10(1)$ & $-3(2)$ \\
\hline C144 & $32(2)$ & $17(2)$ & $30(2)$ & $5(1)$ & $16(2)$ & $0(1)$ \\
\hline $\mathrm{C} 12$ & $20(2)$ & $15(2)$ & $33(2)$ & $0(1)$ & $7(1)$ & $-3(1)$ \\
\hline C62 & $23(2)$ & $15(2)$ & $34(2)$ & $-5(1)$ & $10(1)$ & $-8(1)$ \\
\hline $\mathrm{C} 13$ & $22(2)$ & $14(2)$ & $49(2)$ & $-2(1)$ & $20(2)$ & $0(1)$ \\
\hline C63 & $33(2)$ & $18(2)$ & $51(2)$ & $-3(2)$ & $29(2)$ & $0(1)$ \\
\hline $\mathrm{C} 23$ & $22(2)$ & $24(2)$ & $54(2)$ & $5(2)$ & $15(2)$ & $1(2)$ \\
\hline C134 & $30(2)$ & 19(2) & $32(2)$ & $2(1)$ & $17(1)$ & $1(1)$ \\
\hline $\mathrm{C} 24$ & $33(2)$ & $15(2)$ & $26(2)$ & $-3(1)$ & $15(1)$ & $3(1)$ \\
\hline C41 & $25(2)$ & $22(2)$ & $32(2)$ & $2(1)$ & $13(1)$ & $4(1)$ \\
\hline $\mathrm{C} 21$ & $28(2)$ & $18(2)$ & $28(2)$ & $-2(1)$ & $14(1)$ & $-4(1)$ \\
\hline $\mathrm{C} 14$ & $26(2)$ & $18(2)$ & $29(2)$ & $-3(1)$ & $16(1)$ & $0(1)$ \\
\hline $\mathrm{C} 42$ & $24(2)$ & $26(2)$ & $48(2)$ & $-1(2)$ & $3(2)$ & $0(2)$ \\
\hline C154 & $31(2)$ & $30(2)$ & $32(2)$ & $-2(2)$ & $16(2)$ & $-5(2)$ \\
\hline C174 & $30(2)$ & $43(2)$ & $42(2)$ & $4(2)$ & $15(2)$ & $-10(2)$ \\
\hline C64 & $34(2)$ & $22(2)$ & $38(2)$ & $-5(2)$ & $8(2)$ & $1(2)$ \\
\hline C84 & $24(2)$ & $17(2)$ & $29(2)$ & $-2(1)$ & $14(1)$ & $-4(1)$ \\
\hline C32 & $33(2)$ & $27(2)$ & $35(2)$ & $1(2)$ & $5(2)$ & $-4(2)$ \\
\hline $\mathrm{C} 112$ & $50(2)$ & $32(2)$ & $48(2)$ & $-7(2)$ & $29(2)$ & $-8(2)$ \\
\hline $\mathrm{C} 103$ & $45(2)$ & $28(2)$ & $39(2)$ & $-2(2)$ & $28(2)$ & $-5(2)$ \\
\hline C54 & $50(2)$ & $29(2)$ & $31(2)$ & $4(2)$ & $12(2)$ & $8(2)$ \\
\hline C104 & $31(2)$ & $20(2)$ & $41(2)$ & $-7(2)$ & $17(2)$ & $1(2)$ \\
\hline C102 & $25(2)$ & $33(2)$ & $37(2)$ & $-3(2)$ & $14(2)$ & $0(2)$ \\
\hline C124 & $34(2)$ & $28(2)$ & $27(2)$ & $2(1)$ & $14(1)$ & $-2(2)$ \\
\hline
\end{tabular}




\begin{tabular}{lcccccc} 
C43 & $31(2)$ & $35(2)$ & $84(3)$ & $-1(2)$ & $37(2)$ & $0(2)$ \\
C184 & $38(2)$ & $27(2)$ & $42(2)$ & $3(2)$ & $25(2)$ & $2(2)$ \\
C51 & $30(2)$ & $19(2)$ & $66(2)$ & $4(2)$ & $25(2)$ & $5(2)$ \\
C11 & $29(2)$ & $21(2)$ & $65(2)$ & $3(2)$ & $12(2)$ & $-4(2)$ \\
C82 & $57(2)$ & $38(2)$ & $50(2)$ & $6(2)$ & $33(2)$ & $1(2)$ \\
C72 & $39(2)$ & $37(2)$ & $30(2)$ & $4(2)$ & $17(2)$ & $8(2)$ \\
C33 & $23(2)$ & $30(2)$ & $75(3)$ & $7(2)$ & $10(2)$ & $3(2)$ \\
C194 & $31(2)$ & $18(2)$ & $35(2)$ & $-1(1)$ & $16(2)$ & $-3(1)$ \\
C44 & $52(2)$ & $30(2)$ & $33(2)$ & $7(2)$ & $22(2)$ & $3(2)$ \\
C164 & $41(2)$ & $39(2)$ & $36(2)$ & $-4(2)$ & $18(2)$ & $-14(2)$ \\
C83 & $34(2)$ & $68(3)$ & $36(2)$ & $17(2)$ & $10(2)$ & $5(2)$ \\
C122 & $131(4)$ & $29(2)$ & $45(2)$ & $-3(2)$ & $41(3)$ & $-17(3)$ \\
C73 & $24(2)$ & $43(2)$ & $51(2)$ & $16(2)$ & $0(2)$ & $-8(2)$ \\
C53 & $38(2)$ & $27(2)$ & $61(2)$ & $-8(2)$ & $36(2)$ & $-2(2)$ \\
C52 & $23(2)$ & $25(2)$ & $44(2)$ & $-7(2)$ & $11(1)$ & $-7(2)$ \\
C93 & $52(3)$ & $47(3)$ & $70(3)$ & $17(2)$ & $-9(2)$ & $1(2)$ \\
C123 & $70(3)$ & $51(3)$ & $49(2)$ & $5(2)$ & $29(2)$ & $-12(2)$ \\
C113 & $76(3)$ & $46(3)$ & $58(3)$ & $-17(2)$ & $27(2)$ & $-3(2)$ \\
C92 & $83(3)$ & $42(3)$ & $89(3)$ & $-20(2)$ & $53(3)$ & $-4(2)$ \\
C15 & $114(9)$ & $340(20)$ & $43(5)$ & $30(9)$ & $14(5)$ & $-29(11)$ \\
C25 & $138(10)$ & $51(6)$ & $34(5)$ & $-9(4)$ & $21(5)$ & $5(6)$ \\
O15 & $90(6)$ & $185(11)$ & $81(5)$ & $-50(6)$ & $29(5)$ & $14(7)$ \\
C35 & $99(8)$ & $53(6)$ & $29(4)$ & $-4(4)$ & $6(4)$ & $-31(6)$ \\
C45 & $114(9)$ & $340(20)$ & $43(5)$ & $30(9)$ & $14(5)$ & $-29(11)$ \\
& & & & & & \\
\hline
\end{tabular}


Table S-5. Hydrogen coordinates (x 10 $)$ and isotropic displacement parameters $\left(\AA^{2} \times 10^{3}\right)$ for $\mathrm{L}^{\mathrm{Me}} \mathrm{Fe}\left(\mathrm{CPh}_{3}\right]$ (1).

\begin{tabular}{|c|c|c|c|c|}
\hline & $\mathrm{x}$ & $\mathrm{y}$ & $\mathrm{z}$ & $\mathrm{U}(\mathrm{eq})$ \\
\hline H31 & 7150 & 6549 & 4110 & 30 \\
\hline H114 & 8189 & -492 & 2124 & 36 \\
\hline H74 & 8573 & 522 & 4644 & 31 \\
\hline H34 & 6838 & -1262 & 4838 & 35 \\
\hline H94 & 8158 & -1473 & 3847 & 30 \\
\hline H134 & 7055 & 1711 & 2963 & 31 \\
\hline $\mathrm{H} 42$ & 10416 & 1953 & 5687 & 42 \\
\hline H154 & 6501 & -1182 & 2995 & 36 \\
\hline H174 & 4540 & -1275 & 3159 & 45 \\
\hline H64 & 9243 & -319 & 5599 & 38 \\
\hline H32 & 9608 & 2537 & 6195 & 40 \\
\hline H11A & 9712 & 1905 & 3605 & 60 \\
\hline H11B & 9029 & 2118 & 3009 & 60 \\
\hline $\mathrm{H} 11 \mathrm{C}$ & 8985 & 1184 & 3568 & 60 \\
\hline H103 & 6673 & 2728 & 5185 & 41 \\
\hline H54 & 8720 & -1622 & 6191 & 44 \\
\hline H104 & 8565 & -1836 & 3006 & 35 \\
\hline H102 & 8463 & 3310 & 3585 & 37 \\
\hline H124 & 7450 & 1314 & 2115 & 34 \\
\hline $\mathrm{H} 43$ & 3934 & 2276 & 4187 & 55 \\
\hline H184 & 4962 & -66 & 4089 & 39 \\
\hline H51A & 5542 & 5876 & 3685 & 54 \\
\hline H51B & 6081 & 6901 & 4134 & 54 \\
\hline $\mathrm{H} 51 \mathrm{C}$ & 5701 & 5791 & 4422 & 54 \\
\hline H11D & 8862 & 5556 & 4861 & 58 \\
\hline H11E & 8324 & 6766 & 4801 & 58 \\
\hline $\mathrm{H} 11 \mathrm{~F}$ & 8548 & 6317 & 4211 & 58 \\
\hline H82A & 7951 & 1646 & 5881 & 67 \\
\hline H82B & 7864 & 2675 & 6391 & 67 \\
\hline $\mathrm{H} 82 \mathrm{C}$ & 8626 & 2077 & 6449 & 67 \\
\hline H72 & 7847 & 3763 & 5459 & 41 \\
\hline
\end{tabular}




\begin{tabular}{|c|c|c|c|c|}
\hline H33 & 4082 & 2884 & 3247 & 53 \\
\hline H194 & 6117 & 637 & 4443 & 32 \\
\hline H44 & 7514 & -2095 & 5795 & 44 \\
\hline H164 & 5335 & -1866 & 2630 & 45 \\
\hline $\mathrm{H} 83 \mathrm{~A}$ & 5649 & 1752 & 2873 & 69 \\
\hline H83B & 5364 & 2527 & 2224 & 69 \\
\hline $\mathrm{H} 83 \mathrm{C}$ & 4817 & 1848 & 2519 & 69 \\
\hline $\mathrm{H} 12 \mathrm{~A}$ & 9176 & 5201 & 3792 & 98 \\
\hline H12B & 9254 & 4492 & 3184 & 98 \\
\hline $\mathrm{H} 12 \mathrm{C}$ & 9862 & 4331 & 3839 & 98 \\
\hline $\mathrm{H} 73$ & 5702 & 4061 & 3047 & 51 \\
\hline $\mathrm{H} 53$ & 4880 & 2292 & 5091 & 45 \\
\hline H52 & 10136 & 2276 & 4622 & 36 \\
\hline H93A & 4207 & 4100 & 2439 & 95 \\
\hline H93B & 4784 & 4777 & 2180 & 95 \\
\hline $\mathrm{H} 93 \mathrm{C}$ & 4650 & 5353 & 2792 & 95 \\
\hline $\mathrm{H} 12 \mathrm{D}$ & 5816 & 1978 & 5922 & 82 \\
\hline $\mathrm{H} 12 \mathrm{E}$ & 6648 & 1733 & 6089 & 82 \\
\hline $\mathrm{H} 12 \mathrm{~F}$ & 6107 & 948 & 5522 & 82 \\
\hline $\mathrm{H} 11 \mathrm{G}$ & 6406 & 4928 & 5341 & 88 \\
\hline $\mathrm{H} 11 \mathrm{H}$ & 6781 & 4213 & 5994 & 88 \\
\hline H11I & 5945 & 4452 & 5767 & 88 \\
\hline $\mathrm{H} 92 \mathrm{~A}$ & 9028 & 4526 & 6502 & 98 \\
\hline H92B & 8231 & 4996 & 6391 & 98 \\
\hline H92C & 8645 & 5478 & 5929 & 98 \\
\hline $\mathrm{H} 15 \mathrm{~A}$ & 7482 & 3296 & 7873 & 252 \\
\hline H15B & 6977 & 4067 & 7281 & 252 \\
\hline $\mathrm{H} 15 \mathrm{C}$ & 7094 & 4635 & 7966 & 252 \\
\hline $\mathrm{H} 25 \mathrm{~A}$ & 8303 & 5125 & 8068 & 91 \\
\hline $\mathrm{H} 25 \mathrm{~B}$ & 8176 & 4572 & 7369 & 91 \\
\hline $\mathrm{H} 35 \mathrm{~A}$ & 8290 & 7218 & 7093 & 76 \\
\hline H35B & 8381 & 7490 & 7818 & 76 \\
\hline $\mathrm{H} 45 \mathrm{~A}$ & 7702 & 9250 & 7191 & 252 \\
\hline $\mathrm{H} 45 \mathrm{~B}$ & 7229 & 8447 & 7525 & 252 \\
\hline $\mathrm{H} 45 \mathrm{C}$ & 7140 & 8178 & 6807 & 252 \\
\hline
\end{tabular}


Table S-6. Torsion angles $\left[^{\circ}\right]$ for $\mathrm{L}^{\mathrm{Me}} \mathrm{Fe}\left(\mathrm{CPh}_{3}\right]$ (1).

\begin{tabular}{|c|c|c|c|}
\hline N11-Fe1-N21-C41 & $15.0(2)$ & $\mathrm{C} 44-\mathrm{C} 34-\mathrm{C} 24-\mathrm{C} 14$ & $-179.2(3)$ \\
\hline C14-Fe1-N21-C41 & $-164.9(2)$ & C13-N21-C41-C31 & $172.6(3)$ \\
\hline N11-Fe1-N21-C13 & $-161.9(2)$ & Fe1-N21-C41-C31 & $-4.6(4)$ \\
\hline C14-Fe1-N21-C13 & $18.1(3)$ & C13-N21-C41-C51 & $-4.9(4)$ \\
\hline N21-Fe1-N11-C21 & $-15.3(2)$ & Fe1-N21-C41-C51 & $178.0(2)$ \\
\hline C14-Fe1-N11-C21 & $164.6(2)$ & $\mathrm{C} 21-\mathrm{C} 31-\mathrm{C} 41-\mathrm{N} 21$ & $-13.2(5)$ \\
\hline N21-Fe1-N11-C12 & $155.0(2)$ & C21-C31-C41-C51 & $164.4(3)$ \\
\hline C14-Fe1-N11-C12 & $-25.0(3)$ & C12-N11-C21-C31 & $-165.5(3)$ \\
\hline C32-C22-C12-C62 & $-1.2(4)$ & Fe1-N11-C21-C31 & $4.9(4)$ \\
\hline $\mathrm{C} 72-\mathrm{C} 22-\mathrm{C} 12-\mathrm{C} 62$ & $177.6(3)$ & C12-N11-C21-C11 & $11.8(4)$ \\
\hline C32-C22-C12-N11 & $177.2(3)$ & Fe1-N11-C21-C11 & $-177.8(2)$ \\
\hline C72-C22-C12-N11 & $-4.1(4)$ & C41-C31-C21-N11 & $13.0(5)$ \\
\hline C21-N11-C12-C62 & $-95.5(3)$ & $\mathrm{C} 41-\mathrm{C} 31-\mathrm{C} 21-\mathrm{C} 11$ & $-164.5(3)$ \\
\hline Fe1-N11-C12-C62 & $93.6(3)$ & C154-C144-C14-C24 & $-115.2(3)$ \\
\hline C21-N11-C12-C22 & $86.1(3)$ & C194-C144-C14-C24 & $65.9(4)$ \\
\hline Fe1-N11-C12-C22 & $-84.7(3)$ & C154-C144-C14-C84 & $12.7(4)$ \\
\hline C22-C12-C62-C52 & $1.1(4)$ & C194-C144-C14-C84 & $-166.1(3)$ \\
\hline N11-C12-C62-C52 & $-177.2(3)$ & C154-C144-C14-Fe1 & $129.4(3)$ \\
\hline $\mathrm{C} 22-\mathrm{C} 12-\mathrm{C} 62-\mathrm{C} 102$ & $-177.5(3)$ & C194-C144-C14-Fe1 & $-49.5(3)$ \\
\hline N11-C12-C62-C102 & $4.1(4)$ & C74-C24-C14-C144 & $172.3(3)$ \\
\hline C41-N21-C13-C23 & $81.1(4)$ & C34-C24-C14-C144 & $-7.8(4)$ \\
\hline Fe1-N21-C13-C23 & $-101.8(3)$ & C74-C24-C14-C84 & $46.6(4)$ \\
\hline C41-N21-C13-C63 & $-99.4(3)$ & C34-C24-C14-C84 & $-133.5(3)$ \\
\hline Fe1-N21-C13-C63 & $77.8(3)$ & C74-C24-C14-Fe1 & $-70.1(3)$ \\
\hline C23-C13-C63-C53 & $-1.4(5)$ & $\mathrm{C} 34-\mathrm{C} 24-\mathrm{C} 14-\mathrm{Fe} 1$ & $109.8(3)$ \\
\hline N21-C13-C63-C53 & $179.0(3)$ & N11-Fe1-C14-C144 & $-175.31(16)$ \\
\hline C23-C13-C63-C103 & 178.1(3) & N21-Fe1-C14-C144 & $4.6(3)$ \\
\hline N21-C13-C63-C103 & $-1.4(4)$ & N11-Fe1-C14-C24 & $63.3(2)$ \\
\hline C63-C13-C23-C33 & $1.3(5)$ & N21-Fe1-C14-C24 & $-116.8(2)$ \\
\hline N21-C13-C23-C33 & $-179.2(3)$ & N11-Fe1-C14-C84 & $-57.5(2)$ \\
\hline C63-C13-C23-C73 & $-175.2(3)$ & N21-Fe1-C14-C84 & $122.4(2)$ \\
\hline N21-C13-C23-C73 & $4.3(5)$ & C194-C144-C154-C164 & $1.1(5)$ \\
\hline C64-C74-C24-C34 & $-0.6(4)$ & C14-C144-C154-C164 & $-177.8(3)$ \\
\hline C64-C74-C24-C14 & $179.3(3)$ & C24-C74-C64-C54 & $0.0(5)$ \\
\hline C44-C34-C24-C74 & $0.7(4)$ & C124-C134-C84-C94 & $1.3(4)$ \\
\hline
\end{tabular}




\begin{tabular}{|c|c|c|c|}
\hline C124-C134-C84-C14 & $-171.3(3)$ & C24-C34-C44-C54 & $-0.1(5)$ \\
\hline C104-C94-C84-C134 & $-0.7(4)$ & C144-C154-C164-C174 & $0.0(5)$ \\
\hline C104-C94-C84-C14 & $171.7(3)$ & C184-C174-C164-C154 & $-1.5(5)$ \\
\hline C144-C14-C84-C134 & $72.2(3)$ & $\mathrm{C} 13-\mathrm{C} 23-\mathrm{C} 73-\mathrm{C} 83$ & $100.0(4)$ \\
\hline C24-C14-C84-C134 & $-159.7(3)$ & $\mathrm{C} 33-\mathrm{C} 23-\mathrm{C} 73-\mathrm{C} 83$ & $-76.5(4)$ \\
\hline Fe1-C14-C84-C134 & $-44.9(3)$ & $\mathrm{C} 13-\mathrm{C} 23-\mathrm{C} 73-\mathrm{C} 93$ & $-135.1(3)$ \\
\hline C144-C14-C84-C94 & $-99.9(3)$ & $\mathrm{C} 33-\mathrm{C} 23-\mathrm{C} 73-\mathrm{C} 93$ & $48.5(5)$ \\
\hline C24-C14-C84-C94 & $28.1(4)$ & C33-C43-C53-C63 & $0.1(6)$ \\
\hline Fe1-C14-C84-C94 & $143.0(3)$ & C13-C63-C53-C43 & $0.7(5)$ \\
\hline $\mathrm{C} 52-\mathrm{C} 42-\mathrm{C} 32-\mathrm{C} 22$ & $1.4(5)$ & C103-C63-C53-C43 & $-178.9(3)$ \\
\hline $\mathrm{C} 12-\mathrm{C} 22-\mathrm{C} 32-\mathrm{C} 42$ & $-0.1(5)$ & C32-C42-C52-C62 & $-1.5(5)$ \\
\hline $\mathrm{C} 72-\mathrm{C} 22-\mathrm{C} 32-\mathrm{C} 42$ & $-178.9(3)$ & $\mathrm{C} 12-\mathrm{C} 62-\mathrm{C} 52-\mathrm{C} 42$ & $0.3(5)$ \\
\hline C53-C63-C103-C113 & $-93.3(4)$ & $\mathrm{C} 102-\mathrm{C} 62-\mathrm{C} 52-\mathrm{C} 42$ & $178.9(3)$ \\
\hline C13-C63-C103-C113 & $87.2(4)$ & $\mathrm{C} 15-\mathrm{C} 25-\mathrm{O} 15-\mathrm{C} 35$ & $168.9(9)$ \\
\hline C53-C63-C103-C123 & $33.9(4)$ & $\mathrm{C} 25-\mathrm{O} 15-\mathrm{C} 35-\mathrm{C} 45$ & $-171.1(9)$ \\
\hline C13-C63-C103-C123 & $-145.7(3)$ & & \\
\hline C74-C64-C54-C44 & $0.6(5)$ & & \\
\hline C124-C114-C104-C94 & $2.0(5)$ & & \\
\hline C84-C94-C104-C114 & $-1.0(5)$ & & \\
\hline C52-C62-C102-C112 & $47.1(4)$ & & \\
\hline C12-C62-C102-C112 & $-134.3(3)$ & & \\
\hline C52-C62-C102-C122 & $-75.4(4)$ & & \\
\hline C12-C62-C102-C122 & $103.2(4)$ & & \\
\hline C104-C114-C124-C134 & $-1.4(5)$ & & \\
\hline C84-C134-C124-C114 & $-0.3(5)$ & & \\
\hline C164-C174-C184-C194 & $2.0(5)$ & & \\
\hline C32-C22-C72-C82 & $-55.9(4)$ & & \\
\hline $\mathrm{C} 12-\mathrm{C} 22-\mathrm{C} 72-\mathrm{C} 82$ & $125.4(3)$ & & \\
\hline C32-C22-C72-C92 & $67.8(4)$ & & \\
\hline $\mathrm{C} 12-\mathrm{C} 22-\mathrm{C} 72-\mathrm{C} 92$ & $-110.9(4)$ & & \\
\hline C53-C43-C33-C23 & $-0.2(6)$ & & \\
\hline $\mathrm{C} 13-\mathrm{C} 23-\mathrm{C} 33-\mathrm{C} 43$ & $-0.5(5)$ & & \\
\hline C73-C23-C33-C43 & $176.1(3)$ & & \\
\hline C174-C184-C194-C144 & $-1.0(5)$ & & \\
\hline C154-C144-C194-C184 & $-0.6(5)$ & & \\
\hline C14-C144-C194-C184 & $178.4(3)$ & & \\
\hline C64-C54-C44-C34 & $-0.5(5)$ & & \\
\hline
\end{tabular}




\section{$\left[\mathrm{L}^{\mathrm{Me}} \mathrm{Fe}\left(\mathrm{Ph}_{2} \mathrm{CO}\right](2)\right.$}

A crystal $\left(0.28 \times 0.12 \times 0.05 \mathrm{~mm}^{3}\right)$ was placed onto the tip of a $0.1 \mathrm{~mm}$ diameter glass capillary tube or fiber and mounted on a Bruker SMART APEX II CCD platform diffractometer for a data collection at 100.0(5) K. ${ }^{13} \mathrm{~A}$ preliminary set of cell constants and an orientation matrix were calculated from reflections harvested from three orthogonal wedges of reciprocal space. The full data collection was carried out using MoKa radiation (graphite monochromator) with a frame time of 90 seconds and a detector distance of $4.00 \mathrm{~cm}$. A randomly oriented region of reciprocal space was surveyed: three major sections of frames were collected with $0.50^{\circ}$ steps in $\omega$ at three different $\phi$ settings and a detector position of $-38^{\circ}$ in $2 \theta$. The intensity data were corrected for absorption. ${ }^{14}$ Final cell constants were calculated from the xyz centroids of 2904 strong reflections from the actual data collection after integration. ${ }^{15}$ See Table S-7 for additional crystal and refinement information.

The structure was solved using SIR2011 16 and refined using SHELXL-2013. ${ }^{17}$ The space group $C 2 / c$ was determined based on systematic absences and intensity statistics. A direct-methods solution was calculated which provided most non-hydrogen atoms from the E-map. Full-matrix least squares / difference Fourier cycles were performed which located the remaining non-hydrogen atoms. All non-hydrogen atoms were refined with anisotropic displacement parameters. All hydrogen atoms were placed in ideal positions and refined as riding atoms with relative isotropic displacement parameters. The final full matrix least squares refinement converged to $R 1=0.0655$ $\left(F^{2}, I>2 \sigma(I)\right)$ and $w R^{2}=0.1579\left(F^{2}\right.$, all data).

The asymmetric unit contains one iron molecule in a general position and one cocrystallized $n$-hexane solvent molecule in a crystallographic inversion center. The unique portion of the latter is modeled as disordered over two general positions (71:29).

Unless noted otherwise all structural diagrams containing thermal displacement ellipsoids are drawn at the $50 \%$ probability level. 


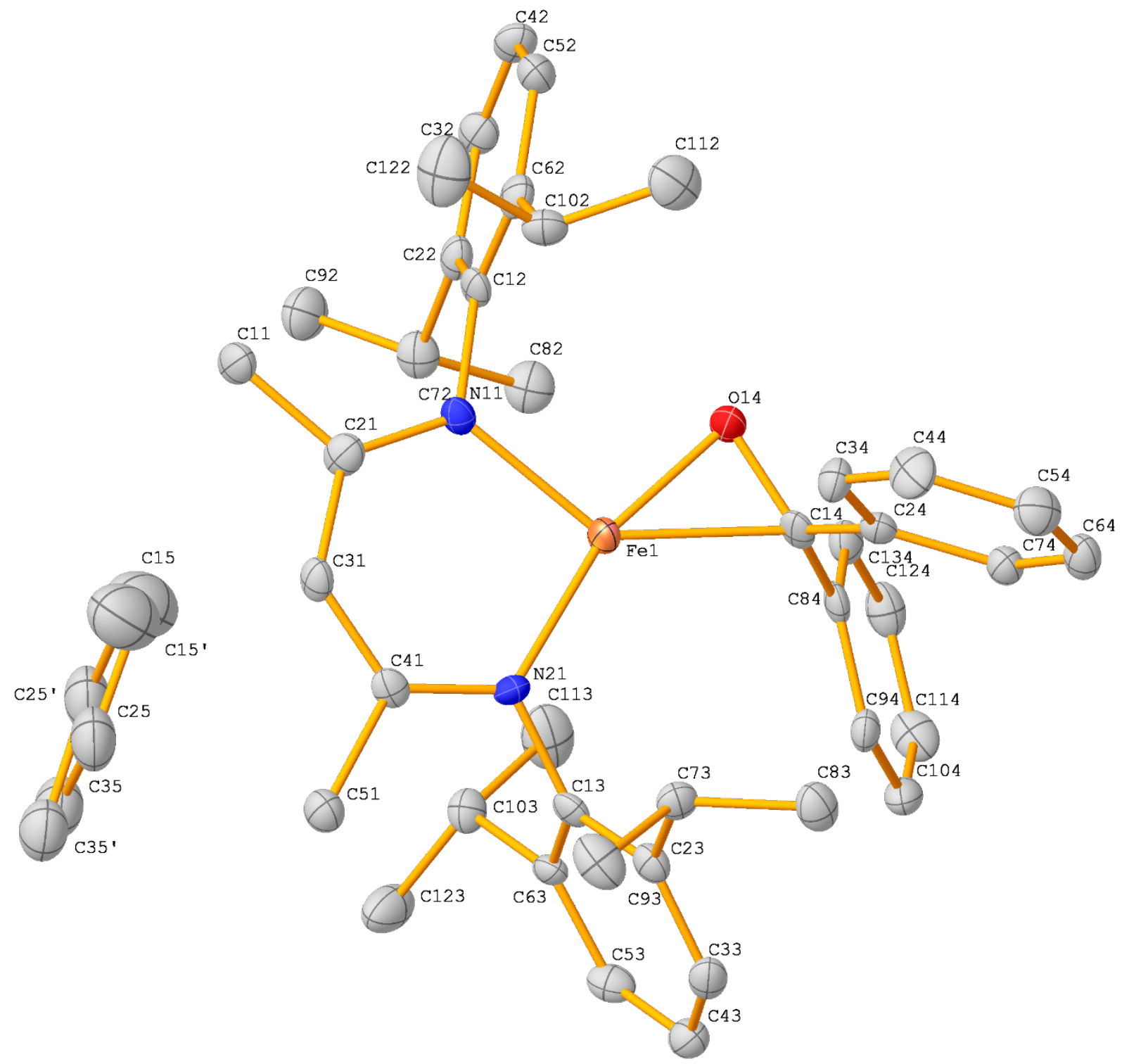

Figure S-25: The complete numbering scheme of $\mathrm{L}^{\mathrm{Me}} \mathrm{Fe}\left(\mathrm{Ph}_{2} \mathrm{CO}\right)$ (2) with 50\% thermal ellipsoid probability levels. The hydrogen atoms are omitted for clarity. 
Table S-7. Crystal data and structure refinement for $\mathrm{L}^{\mathrm{Me}} \mathrm{Fe}\left(\mathrm{Ph}_{2} \mathrm{CO}\right)$ (2).

\begin{tabular}{|c|c|c|}
\hline Identification code & \multicolumn{2}{|l|}{ holkcm09 } \\
\hline CSD Deposition Number & \multicolumn{2}{|l|}{1944809} \\
\hline Empirical formula & \multicolumn{2}{|l|}{ C45 H58 Fe N2 O } \\
\hline Formula weight & \multicolumn{2}{|l|}{698.78} \\
\hline Temperature & \multicolumn{2}{|l|}{$100.0(5) \mathrm{K}$} \\
\hline Wavelength & \multicolumn{2}{|l|}{$0.71073 \AA$} \\
\hline Crystal system & \multicolumn{2}{|l|}{ monoclinic } \\
\hline Space group & \multicolumn{2}{|l|}{$C 2 / c$} \\
\hline \multirow[t]{3}{*}{ Unit cell dimensions } & $a=39.564(5) \AA$ & $\alpha=90^{\circ}$ \\
\hline & $b=11.3678(13) \AA$ & $\beta=107.263(2)^{\circ}$ \\
\hline & $c=17.931(2) \AA$ & $\gamma=90^{\circ}$ \\
\hline Volume & \multicolumn{2}{|l|}{$7701.2(16) \AA^{3}$} \\
\hline$Z$ & \multicolumn{2}{|c|}{8} \\
\hline Density (calculated) & \multicolumn{2}{|l|}{$1.205 \mathrm{~g} / \mathrm{cm}^{3}$} \\
\hline Absorption coefficient & \multicolumn{2}{|l|}{$0.428 \mathrm{~mm}^{-1}$} \\
\hline$F(000)$ & \multicolumn{2}{|l|}{3008} \\
\hline Crystal color, morphology & \multicolumn{2}{|l|}{ orange, plate } \\
\hline Crystal size & \multicolumn{2}{|c|}{$0.28 \times 0.12 \times 0.05 \mathrm{~mm}^{3}$} \\
\hline Theta range for data collection & \multicolumn{2}{|l|}{1.078 to $25.086^{\circ}$} \\
\hline Index ranges & \multicolumn{2}{|c|}{$-46 \leq h \leq 46,-13 \leq k \leq 12,-21 \leq l \leq 21$} \\
\hline Reflections collected & \multicolumn{2}{|l|}{29328} \\
\hline Independent reflections & \multicolumn{2}{|c|}{$6852[R(\mathrm{int})=0.1658]$} \\
\hline Observed reflections & \multicolumn{2}{|l|}{3906} \\
\hline Completeness to theta $=25.030^{\circ}$ & \multicolumn{2}{|l|}{$100.0 \%$} \\
\hline Absorption correction & \multicolumn{2}{|l|}{ Multi-scan } \\
\hline Max. and min. transmission & \multicolumn{2}{|l|}{0.7452 and 0.5466} \\
\hline Refinement method & \multicolumn{2}{|c|}{ Full-matrix least-squares on $F^{2}$} \\
\hline Data / restraints / parameters & \multicolumn{2}{|l|}{6852 / 4 / 464} \\
\hline Goodness-of-fit on $F^{2}$ & \multicolumn{2}{|l|}{1.026} \\
\hline Final $R$ indices $[I>2 \operatorname{sigma}(I)]$ & \multicolumn{2}{|c|}{$R 1=0.0655, w R 2=0.1235$} \\
\hline$R$ indices (all data) & \multicolumn{2}{|c|}{$R 1=0.1381, w R 2=0.1579$} \\
\hline Largest diff. peak and hole & \multicolumn{2}{|c|}{0.426 and -0.450 e. $\AA^{-3}$} \\
\hline
\end{tabular}


Table S-8. Atomic coordinates (x 10 $)$ and equivalent isotropic displacement parameters $\left(\AA^{2} \times 10^{3}\right)$ for 2. $U_{e q}$ is defined as one third of the trace of the orthogonalized $U_{i j}$ tensor.

\begin{tabular}{|c|c|c|c|c|}
\hline & $\mathrm{x}$ & $\mathrm{y}$ & $\mathrm{z}$ & $\mathrm{U}_{\mathrm{eq}}$ \\
\hline $\mathrm{Fe} 1$ & $3564(1)$ & $1298(1)$ & $1246(1)$ & $17(1)$ \\
\hline $\mathrm{O} 14$ & $3490(1)$ & $888(3)$ & $2175(2)$ & $21(1)$ \\
\hline N11 & $3400(1)$ & 2971(3) & $1181(2)$ & $16(1)$ \\
\hline $\mathrm{N} 21$ & $3714(1)$ & $1284(3)$ & $307(2)$ & $16(1)$ \\
\hline $\mathrm{C} 11$ & $3286(1)$ & 4903(4) & $562(3)$ & $25(1)$ \\
\hline $\mathrm{C} 21$ & 3393(1) & $3644(4)$ & $567(2)$ & $22(1)$ \\
\hline $\mathrm{C} 31$ & $3496(1)$ & $3245(4)$ & $-72(2)$ & $20(1)$ \\
\hline $\mathrm{C} 41$ & $3652(1)$ & 2194(4) & $-188(2)$ & $17(1)$ \\
\hline $\mathrm{C} 51$ & $3773(1)$ & $2128(4)$ & $-909(3)$ & $25(1)$ \\
\hline $\mathrm{C} 12$ & $3311(1)$ & $3477(4)$ & $1837(2)$ & $18(1)$ \\
\hline $\mathrm{C} 22$ & $3581(1)$ & $4010(4)$ & $2435(3)$ & $21(1)$ \\
\hline $\mathrm{C} 32$ & $3488(1)$ & $4455(4)$ & $3074(2)$ & $22(1)$ \\
\hline $\mathrm{C} 42$ & $3151(1)$ & 4397(4) & $3116(3)$ & $25(1)$ \\
\hline $\mathrm{C} 52$ & $2890(1)$ & $3852(4)$ & 2532(3) & $25(1)$ \\
\hline C62 & $2967(1)$ & $3382(4)$ & 1883(3) & $22(1)$ \\
\hline $\mathrm{C} 72$ & 3961(1) & 4079(4) & $2424(3)$ & $23(1)$ \\
\hline C82 & 4191(1) & $3212(4)$ & $3012(3)$ & $30(1)$ \\
\hline C92 & $4112(1)$ & $5318(4)$ & $2571(3)$ & $33(1)$ \\
\hline $\mathrm{C} 102$ & $2675(1)$ & 2755(4) & $1263(3)$ & $22(1)$ \\
\hline $\mathrm{C} 112$ & $2487(1)$ & $1836(4)$ & 1611(3) & $33(1)$ \\
\hline $\mathrm{C} 122$ & $2403(2)$ & $3635(5)$ & $777(3)$ & $42(2)$ \\
\hline $\mathrm{C} 13$ & $3930(1)$ & $328(4)$ & $186(2)$ & $18(1)$ \\
\hline $\mathrm{C} 23$ & $3769(1)$ & $-729(4)$ & $-158(3)$ & $20(1)$ \\
\hline $\mathrm{C} 33$ & $3988(1)$ & $-1633(4)$ & $-257(2)$ & $22(1)$ \\
\hline $\mathrm{C} 43$ & 4353(1) & $-1521(4)$ & $-14(3)$ & $23(1)$ \\
\hline $\mathrm{C} 53$ & $4504(1)$ & $-487(4)$ & $331(3)$ & $22(1)$ \\
\hline C63 & $4298(1)$ & $454(4)$ & $429(2)$ & $18(1)$ \\
\hline $\mathrm{C} 73$ & $3374(1)$ & $-901(4)$ & $-422(3)$ & $20(1)$ \\
\hline $\mathrm{C} 83$ & $3270(1)$ & $-2114(4)$ & $-188(3)$ & $26(1)$ \\
\hline C93 & $3224(1)$ & $-753(5)$ & $-1304(3)$ & $33(1)$ \\
\hline $\mathrm{C} 103$ & $4475(1)$ & $1576(4)$ & $826(3)$ & $22(1)$ \\
\hline $\mathrm{C} 113$ & $4576(2)$ & 1439(5) & $1706(3)$ & $39(2)$ \\
\hline
\end{tabular}




\begin{tabular}{lrrrr} 
C123 & $4799(1)$ & $1915(4)$ & $577(3)$ & $36(1)$ \\
C14 & $3579(1)$ & $-172(4)$ & $1939(2)$ & $19(1)$ \\
C24 & $3285(1)$ & $-1033(4)$ & $1682(2)$ & $18(1)$ \\
C34 & $2950(1)$ & $-700(4)$ & $1218(3)$ & $23(1)$ \\
C44 & $2673(1)$ & $-1490(4)$ & $1012(3)$ & $30(1)$ \\
C54 & $2725(1)$ & $-2638(4)$ & $1286(3)$ & $28(1)$ \\
C64 & $3052(1)$ & $-2980(4)$ & $1756(3)$ & $24(1)$ \\
C74 & $3330(1)$ & $-2199(4)$ & $1956(3)$ & $21(1)$ \\
C84 & $3944(1)$ & $-591(4)$ & $2296(2)$ & $18(1)$ \\
C94 & $4103(1)$ & $-1398(4)$ & $1924(2)$ & $19(1)$ \\
C104 & $4441(1)$ & $-1786(4)$ & $2274(3)$ & $25(1)$ \\
C114 & $4630(1)$ & $-1388(5)$ & $3008(3)$ & $28(1)$ \\
C124 & $4479(1)$ & $-580(4)$ & $3381(3)$ & $26(1)$ \\
C134 & $4140(1)$ & $-168(4)$ & $3038(3)$ & $22(1)$ \\
C15 & $4385(3)$ & $5200(20)$ & $816(9)$ & $65(5)$ \\
C25 & $4527(2)$ & $4944(7)$ & $123(5)$ & $44(2)$ \\
C35 & $4925(2)$ & $5157(8)$ & $328(4)$ & $41(2)$ \\
C15' & $4310(8)$ & $5210(70)$ & $550(30)$ & $65(5)$ \\
C25' & $4711(6)$ & $5369(17)$ & $676(11)$ & $44(2)$ \\
C35' & $4803(2)$ & $5050(20)$ & $-78(11)$ & $41(2)$ \\
& & & & \\
\hline & & & &
\end{tabular}


Table S-9. Bond lengths $[\AA ̊]$ and angles $\left[{ }^{\circ}\right]$ for $\mathbf{2}$.

\begin{tabular}{|c|c|c|c|}
\hline $\mathrm{Fe}(1)-\mathrm{O}(14)$ & $1.836(3)$ & $\mathrm{C}(82)-\mathrm{H}(82 \mathrm{~B})$ & 0.9800 \\
\hline $\mathrm{Fe}(1)-\mathrm{N}(21)$ & $1.945(4)$ & $\mathrm{C}(82)-\mathrm{H}(82 \mathrm{C})$ & 0.9800 \\
\hline $\mathrm{Fe}(1)-\mathrm{N}(11)$ & $2.001(4)$ & $\mathrm{C}(92)-\mathrm{H}(92 \mathrm{~A})$ & 0.9800 \\
\hline $\mathrm{Fe}(1)-\mathrm{C}(14)$ & $2.073(4)$ & $\mathrm{C}(92)-\mathrm{H}(92 \mathrm{~B})$ & 0.9800 \\
\hline $\mathrm{O}(14)-\mathrm{C}(14)$ & $1.358(5)$ & $\mathrm{C}(92)-\mathrm{H}(92 \mathrm{C})$ & 0.9800 \\
\hline $\mathrm{N}(11)-\mathrm{C}(21)$ & $1.335(5)$ & $\mathrm{C}(102)-\mathrm{C}(112)$ & $1.520(7)$ \\
\hline $\mathrm{N}(11)-\mathrm{C}(12)$ & $1.443(5)$ & $\mathrm{C}(102)-\mathrm{C}(122)$ & $1.535(7)$ \\
\hline $\mathrm{N}(21)-\mathrm{C}(41)$ & $1.337(5)$ & $\mathrm{C}(102)-\mathrm{H}(10 \mathrm{~A})$ & 1.0000 \\
\hline $\mathrm{N}(21)-\mathrm{C}(13)$ & $1.440(5)$ & $\mathrm{C}(112)-\mathrm{H}(11 \mathrm{D})$ & 0.9800 \\
\hline$C(11)-C(21)$ & $1.492(6)$ & $\mathrm{C}(112)-\mathrm{H}(11 \mathrm{E})$ & 0.9800 \\
\hline $\mathrm{C}(11)-\mathrm{H}(11 \mathrm{~A})$ & 0.9800 & $\mathrm{C}(112)-\mathrm{H}(11 \mathrm{~F})$ & 0.9800 \\
\hline $\mathrm{C}(11)-\mathrm{H}(11 \mathrm{~B})$ & 0.9800 & $\mathrm{C}(122)-\mathrm{H}(12 \mathrm{~A})$ & 0.9800 \\
\hline $\mathrm{C}(11)-\mathrm{H}(11 \mathrm{C})$ & 0.9800 & $\mathrm{C}(122)-\mathrm{H}(12 \mathrm{~B})$ & 0.9800 \\
\hline$C(21)-C(31)$ & $1.400(6)$ & $\mathrm{C}(122)-\mathrm{H}(12 \mathrm{C})$ & 0.9800 \\
\hline$C(31)-C(41)$ & $1.390(6)$ & $\mathrm{C}(13)-\mathrm{C}(63)$ & $1.399(7)$ \\
\hline $\mathrm{C}(31)-\mathrm{H}(31 \mathrm{~A})$ & 0.9500 & $\mathrm{C}(13)-\mathrm{C}(23)$ & $1.413(6)$ \\
\hline$C(41)-C(51)$ & $1.507(6)$ & $C(23)-C(33)$ & $1.389(6)$ \\
\hline $\mathrm{C}(51)-\mathrm{H}(51 \mathrm{~A})$ & 0.9800 & $\mathrm{C}(23)-\mathrm{C}(73)$ & $1.506(6)$ \\
\hline $\mathrm{C}(51)-\mathrm{H}(51 \mathrm{~B})$ & 0.9800 & $\mathrm{C}(33)-\mathrm{C}(43)$ & $1.385(7)$ \\
\hline $\mathrm{C}(51)-\mathrm{H}(51 \mathrm{C})$ & 0.9800 & $\mathrm{C}(33)-\mathrm{H}(33 \mathrm{~A})$ & 0.9500 \\
\hline$C(12)-C(62)$ & $1.393(7)$ & $\mathrm{C}(43)-\mathrm{C}(53)$ & $1.380(6)$ \\
\hline$C(12)-C(22)$ & $1.407(6)$ & $\mathrm{C}(43)-\mathrm{H}(43 \mathrm{~A})$ & 0.9500 \\
\hline$C(22)-C(32)$ & $1.398(6)$ & $\mathrm{C}(53)-\mathrm{C}(63)$ & $1.386(6)$ \\
\hline$C(22)-C(72)$ & $1.511(7)$ & $\mathrm{C}(53)-\mathrm{H}(53 \mathrm{~A})$ & 0.9500 \\
\hline$C(32)-C(42)$ & $1.361(7)$ & $\mathrm{C}(63)-\mathrm{C}(103)$ & $1.525(6)$ \\
\hline $\mathrm{C}(32)-\mathrm{H}(32 \mathrm{~A})$ & 0.9500 & $\mathrm{C}(73)-\mathrm{C}(93)$ & $1.524(6)$ \\
\hline$C(42)-C(52)$ & $1.380(6)$ & $\mathrm{C}(73)-\mathrm{C}(83)$ & $1.533(6)$ \\
\hline $\mathrm{C}(42)-\mathrm{H}(42 \mathrm{~A})$ & 0.9500 & $\mathrm{C}(73)-\mathrm{H}(73 \mathrm{~A})$ & 1.0000 \\
\hline$C(52)-C(62)$ & $1.395(6)$ & $\mathrm{C}(83)-\mathrm{H}(83 \mathrm{~A})$ & 0.9800 \\
\hline $\mathrm{C}(52)-\mathrm{H}(52 \mathrm{~A})$ & 0.9500 & $\mathrm{C}(83)-\mathrm{H}(83 \mathrm{~B})$ & 0.9800 \\
\hline $\mathrm{C}(62)-\mathrm{C}(102)$ & $1.523(6)$ & $\mathrm{C}(83)-\mathrm{H}(83 \mathrm{C})$ & 0.9800 \\
\hline$C(72)-C(92)$ & $1.523(6)$ & $\mathrm{C}(93)-\mathrm{H}(93 \mathrm{~A})$ & 0.9800 \\
\hline $\mathrm{C}(72)-\mathrm{C}(82)$ & $1.530(6)$ & $\mathrm{C}(93)-\mathrm{H}(93 \mathrm{~B})$ & 0.9800 \\
\hline $\mathrm{C}(72)-\mathrm{H}(72 \mathrm{~A})$ & 1.0000 & $\mathrm{C}(93)-\mathrm{H}(93 \mathrm{C})$ & 0.9800 \\
\hline $\mathrm{C}(82)-\mathrm{H}(82 \mathrm{~A})$ & 0.9800 & $\mathrm{C}(103)-\mathrm{C}(113)$ & $1.517(6)$ \\
\hline
\end{tabular}




\begin{tabular}{|c|c|c|c|}
\hline$C(103)-C(123)$ & $1.527(7)$ & $\mathrm{C}(25)-\mathrm{H}(25 \mathrm{~A})$ & 0.9900 \\
\hline $\mathrm{C}(103)-\mathrm{H}(10 \mathrm{~B})$ & 1.0000 & $\mathrm{C}(25)-\mathrm{H}(25 \mathrm{~B})$ & 0.9900 \\
\hline $\mathrm{C}(113)-\mathrm{H}(11 \mathrm{G})$ & 0.9800 & $\mathrm{C}(35)-\mathrm{C}(35) \# 1$ & $1.507(13)$ \\
\hline $\mathrm{C}(113)-\mathrm{H}(11 \mathrm{H})$ & 0.9800 & $\mathrm{C}(35)-\mathrm{H}(35 \mathrm{~A})$ & 0.9900 \\
\hline C(113)-H(11I) & 0.9800 & $\mathrm{C}(35)-\mathrm{H}(35 \mathrm{~B})$ & 0.9900 \\
\hline $\mathrm{C}(123)-\mathrm{H}(12 \mathrm{D})$ & 0.9800 & $\mathrm{C}\left(15^{\prime}\right)-\mathrm{C}\left(25^{\prime}\right)$ & $1.55(2)$ \\
\hline $\mathrm{C}(123)-\mathrm{H}(12 \mathrm{E})$ & 0.9800 & $\mathrm{C}\left(15^{\prime}\right)-\mathrm{H}(15 \mathrm{D})$ & 0.9800 \\
\hline $\mathrm{C}(123)-\mathrm{H}(12 \mathrm{~F})$ & 0.9800 & $\mathrm{C}\left(15^{\prime}\right)-\mathrm{H}(15 \mathrm{E})$ & 0.9800 \\
\hline$C(14)-C(84)$ & $1.474(7)$ & $\mathrm{C}\left(15^{\prime}\right)-\mathrm{H}(15 \mathrm{~F})$ & 0.9800 \\
\hline$C(14)-C(24)$ & $1.483(6)$ & $C\left(25^{\prime}\right)-C\left(35^{\prime}\right)$ & $1.542(17)$ \\
\hline$C(24)-C(34)$ & $1.392(6)$ & $\mathrm{C}\left(25^{\prime}\right)-\mathrm{H}(25 \mathrm{C})$ & 0.9900 \\
\hline$C(24)-C(74)$ & $1.407(6)$ & $\mathrm{C}\left(25^{\prime}\right)-\mathrm{H}(25 \mathrm{D})$ & 0.9900 \\
\hline$C(34)-C(44)$ & $1.379(7)$ & $C\left(35^{\prime}\right)-C\left(35^{\prime}\right) \# 1$ & $1.504(15)$ \\
\hline $\mathrm{C}(34)-\mathrm{H}(34 \mathrm{~A})$ & 0.9500 & $\mathrm{C}\left(35^{\prime}\right)-\mathrm{H}(35 \mathrm{C})$ & 0.9900 \\
\hline$C(44)-C(54)$ & $1.388(7)$ & $\mathrm{C}\left(35^{\prime}\right)-\mathrm{H}(35 \mathrm{D})$ & 0.9900 \\
\hline $\mathrm{C}(44)-\mathrm{H}(44 \mathrm{~A})$ & 0.9500 & $\mathrm{O}(14)-\mathrm{Fe}(1)-\mathrm{N}(21)$ & $162.96(15)$ \\
\hline$C(54)-C(64)$ & $1.375(7)$ & $\mathrm{O}(14)-\mathrm{Fe}(1)-\mathrm{N}(11)$ & $99.05(14)$ \\
\hline $\mathrm{C}(54)-\mathrm{H}(54 \mathrm{~A})$ & 0.9500 & $\mathrm{~N}(21)-\mathrm{Fe}(1)-\mathrm{N}(11)$ & $97.73(15)$ \\
\hline$C(64)-C(74)$ & $1.376(6)$ & $\mathrm{O}(14)-\mathrm{Fe}(1)-\mathrm{C}(14)$ & $40.10(15)$ \\
\hline $\mathrm{C}(64)-\mathrm{H}(64 \mathrm{~A})$ & 0.9500 & $\mathrm{~N}(21)-\mathrm{Fe}(1)-\mathrm{C}(14)$ & $123.42(17)$ \\
\hline $\mathrm{C}(74)-\mathrm{H}(74 \mathrm{~A})$ & 0.9500 & $\mathrm{~N}(11)-\mathrm{Fe}(1)-\mathrm{C}(14)$ & $138.75(16)$ \\
\hline C(84)-C(94) & $1.389(6)$ & $\mathrm{C}(14)-\mathrm{O}(14)-\mathrm{Fe}(1)$ & $79.4(2)$ \\
\hline C(84)-C(134) & $1.412(6)$ & $\mathrm{C}(21)-\mathrm{N}(11)-\mathrm{C}(12)$ & $119.5(4)$ \\
\hline C(94)-C(104) & $1.373(6)$ & $\mathrm{C}(21)-\mathrm{N}(11)-\mathrm{Fe}(1)$ & $121.3(3)$ \\
\hline $\mathrm{C}(94)-\mathrm{H}(94 \mathrm{~A})$ & 0.9500 & $\mathrm{C}(12)-\mathrm{N}(11)-\mathrm{Fe}(1)$ & $119.0(3)$ \\
\hline C(104)-C(114) & $1.383(6)$ & $\mathrm{C}(41)-\mathrm{N}(21)-\mathrm{C}(13)$ & $118.9(4)$ \\
\hline $\mathrm{C}(104)-\mathrm{H}(10 \mathrm{C})$ & 0.9500 & $\mathrm{C}(41)-\mathrm{N}(21)-\mathrm{Fe}(1)$ & $121.8(3)$ \\
\hline $\mathrm{C}(114)-\mathrm{C}(124)$ & $1.374(7)$ & $\mathrm{C}(13)-\mathrm{N}(21)-\mathrm{Fe}(1)$ & $119.0(3)$ \\
\hline $\mathrm{C}(114)-\mathrm{H}(11 \mathrm{~J})$ & 0.9500 & $\mathrm{C}(21)-\mathrm{C}(11)-\mathrm{H}(11 \mathrm{~A})$ & 109.5 \\
\hline$C(124)-C(134)$ & $1.379(7)$ & $\mathrm{C}(21)-\mathrm{C}(11)-\mathrm{H}(11 \mathrm{~B})$ & 109.5 \\
\hline $\mathrm{C}(124)-\mathrm{H}(12 \mathrm{G})$ & 0.9500 & $\mathrm{H}(11 \mathrm{~A})-\mathrm{C}(11)-\mathrm{H}(11 \mathrm{~B})$ & 109.5 \\
\hline $\mathrm{C}(134)-\mathrm{H}(13 \mathrm{~A})$ & 0.9500 & $\mathrm{C}(21)-\mathrm{C}(11)-\mathrm{H}(11 \mathrm{C})$ & 109.5 \\
\hline$C(15)-C(25)$ & $1.536(13)$ & $\mathrm{H}(11 \mathrm{~A})-\mathrm{C}(11)-\mathrm{H}(11 \mathrm{C})$ & 109.5 \\
\hline $\mathrm{C}(15)-\mathrm{H}(15 \mathrm{~A})$ & 0.9800 & $\mathrm{H}(11 \mathrm{~B})-\mathrm{C}(11)-\mathrm{H}(11 \mathrm{C})$ & 109.5 \\
\hline $\mathrm{C}(15)-\mathrm{H}(15 \mathrm{~B})$ & 0.9800 & $\mathrm{~N}(11)-\mathrm{C}(21)-\mathrm{C}(31)$ & $123.3(4)$ \\
\hline $\mathrm{C}(15)-\mathrm{H}(15 \mathrm{C})$ & 0.9800 & $\mathrm{~N}(11)-\mathrm{C}(21)-\mathrm{C}(11)$ & $119.4(4)$ \\
\hline$C(25)-C(35)$ & $1.527(10)$ & $\mathrm{C}(31)-\mathrm{C}(21)-\mathrm{C}(11)$ & $117.2(4)$ \\
\hline
\end{tabular}




\begin{tabular}{|c|c|c|c|}
\hline$C(41)-C(31)-C(21)$ & $130.7(4)$ & $\mathrm{C}(72)-\mathrm{C}(82)-\mathrm{H}(82 \mathrm{~B})$ & 109.5 \\
\hline $\mathrm{C}(41)-\mathrm{C}(31)-\mathrm{H}(31 \mathrm{~A})$ & 114.7 & $\mathrm{H}(82 \mathrm{~A})-\mathrm{C}(82)-\mathrm{H}(82 \mathrm{~B})$ & 109.5 \\
\hline $\mathrm{C}(21)-\mathrm{C}(31)-\mathrm{H}(31 \mathrm{~A})$ & 114.7 & $\mathrm{C}(72)-\mathrm{C}(82)-\mathrm{H}(82 \mathrm{C})$ & 109.5 \\
\hline $\mathrm{N}(21)-\mathrm{C}(41)-\mathrm{C}(31)$ & $124.5(4)$ & $\mathrm{H}(82 \mathrm{~A})-\mathrm{C}(82)-\mathrm{H}(82 \mathrm{C})$ & 109.5 \\
\hline $\mathrm{N}(21)-\mathrm{C}(41)-\mathrm{C}(51)$ & 119.3(4) & $\mathrm{H}(82 \mathrm{~B})-\mathrm{C}(82)-\mathrm{H}(82 \mathrm{C})$ & 109.5 \\
\hline$C(31)-C(41)-C(51)$ & $116.1(4)$ & $\mathrm{C}(72)-\mathrm{C}(92)-\mathrm{H}(92 \mathrm{~A})$ & 109.5 \\
\hline $\mathrm{C}(41)-\mathrm{C}(51)-\mathrm{H}(51 \mathrm{~A})$ & 109.5 & $\mathrm{C}(72)-\mathrm{C}(92)-\mathrm{H}(92 \mathrm{~B})$ & 109.5 \\
\hline $\mathrm{C}(41)-\mathrm{C}(51)-\mathrm{H}(51 \mathrm{~B})$ & 109.5 & $\mathrm{H}(92 \mathrm{~A})-\mathrm{C}(92)-\mathrm{H}(92 \mathrm{~B})$ & 109.5 \\
\hline $\mathrm{H}(51 \mathrm{~A})-\mathrm{C}(51)-\mathrm{H}(51 \mathrm{~B})$ & 109.5 & $\mathrm{C}(72)-\mathrm{C}(92)-\mathrm{H}(92 \mathrm{C})$ & 109.5 \\
\hline $\mathrm{C}(41)-\mathrm{C}(51)-\mathrm{H}(51 \mathrm{C})$ & 109.5 & $\mathrm{H}(92 \mathrm{~A})-\mathrm{C}(92)-\mathrm{H}(92 \mathrm{C})$ & 109.5 \\
\hline $\mathrm{H}(51 \mathrm{~A})-\mathrm{C}(51)-\mathrm{H}(51 \mathrm{C})$ & 109.5 & $\mathrm{H}(92 \mathrm{~B})-\mathrm{C}(92)-\mathrm{H}(92 \mathrm{C})$ & 109.5 \\
\hline H(51B)-C(51)-H(51C) & 109.5 & $\mathrm{C}(112)-\mathrm{C}(102)-\mathrm{C}(62)$ & $112.4(4)$ \\
\hline $\mathrm{C}(62)-\mathrm{C}(12)-\mathrm{C}(22)$ & $121.6(4)$ & $\mathrm{C}(112)-\mathrm{C}(102)-\mathrm{C}(122)$ & $109.6(4)$ \\
\hline $\mathrm{C}(62)-\mathrm{C}(12)-\mathrm{N}(11)$ & $119.7(4)$ & $C(62)-C(102)-C(122)$ & $111.2(4)$ \\
\hline$C(22)-C(12)-N(11)$ & $118.7(4)$ & $\mathrm{C}(112)-\mathrm{C}(102)-\mathrm{H}(10 \mathrm{~A})$ & 107.9 \\
\hline$C(32)-C(22)-C(12)$ & $117.1(5)$ & $\mathrm{C}(62)-\mathrm{C}(102)-\mathrm{H}(10 \mathrm{~A})$ & 107.9 \\
\hline$C(32)-C(22)-C(72)$ & $119.8(4)$ & $\mathrm{C}(122)-\mathrm{C}(102)-\mathrm{H}(10 \mathrm{~A})$ & 107.9 \\
\hline$C(12)-C(22)-C(72)$ & $123.0(4)$ & $\mathrm{C}(102)-\mathrm{C}(112)-\mathrm{H}(11 \mathrm{D})$ & 109.5 \\
\hline$C(42)-C(32)-C(22)$ & $122.0(4)$ & $\mathrm{C}(102)-\mathrm{C}(112)-\mathrm{H}(11 \mathrm{E})$ & 109.5 \\
\hline $\mathrm{C}(42)-\mathrm{C}(32)-\mathrm{H}(32 \mathrm{~A})$ & 119.0 & $\mathrm{H}(11 \mathrm{D})-\mathrm{C}(112)-\mathrm{H}(11 \mathrm{E})$ & 109.5 \\
\hline $\mathrm{C}(22)-\mathrm{C}(32)-\mathrm{H}(32 \mathrm{~A})$ & 119.0 & $\mathrm{C}(102)-\mathrm{C}(112)-\mathrm{H}(11 \mathrm{~F})$ & 109.5 \\
\hline$C(32)-C(42)-C(52)$ & $120.3(4)$ & $\mathrm{H}(11 \mathrm{D})-\mathrm{C}(112)-\mathrm{H}(11 \mathrm{~F})$ & 109.5 \\
\hline $\mathrm{C}(32)-\mathrm{C}(42)-\mathrm{H}(42 \mathrm{~A})$ & 119.8 & $\mathrm{H}(11 \mathrm{E})-\mathrm{C}(112)-\mathrm{H}(11 \mathrm{~F})$ & 109.5 \\
\hline $\mathrm{C}(52)-\mathrm{C}(42)-\mathrm{H}(42 \mathrm{~A})$ & 119.8 & $\mathrm{C}(102)-\mathrm{C}(122)-\mathrm{H}(12 \mathrm{~A})$ & 109.5 \\
\hline $\mathrm{C}(42)-\mathrm{C}(52)-\mathrm{C}(62)$ & $120.4(5)$ & $\mathrm{C}(102)-\mathrm{C}(122)-\mathrm{H}(12 \mathrm{~B})$ & 109.5 \\
\hline $\mathrm{C}(42)-\mathrm{C}(52)-\mathrm{H}(52 \mathrm{~A})$ & 119.8 & $\mathrm{H}(12 \mathrm{~A})-\mathrm{C}(122)-\mathrm{H}(12 \mathrm{~B})$ & 109.5 \\
\hline $\mathrm{C}(62)-\mathrm{C}(52)-\mathrm{H}(52 \mathrm{~A})$ & 119.8 & $\mathrm{C}(102)-\mathrm{C}(122)-\mathrm{H}(12 \mathrm{C})$ & 109.5 \\
\hline$C(12)-C(62)-C(52)$ & $118.6(4)$ & $\mathrm{H}(12 \mathrm{~A})-\mathrm{C}(122)-\mathrm{H}(12 \mathrm{C})$ & 109.5 \\
\hline$C(12)-C(62)-C(102)$ & $122.5(4)$ & $\mathrm{H}(12 \mathrm{~B})-\mathrm{C}(122)-\mathrm{H}(12 \mathrm{C})$ & 109.5 \\
\hline $\mathrm{C}(52)-\mathrm{C}(62)-\mathrm{C}(102)$ & $118.8(4)$ & $C(63)-C(13)-C(23)$ & $121.1(4)$ \\
\hline $\mathrm{C}(22)-\mathrm{C}(72)-\mathrm{C}(92)$ & $112.8(4)$ & $\mathrm{C}(63)-\mathrm{C}(13)-\mathrm{N}(21)$ & $119.1(4)$ \\
\hline$C(22)-C(72)-C(82)$ & $110.3(4)$ & $\mathrm{C}(23)-\mathrm{C}(13)-\mathrm{N}(21)$ & $119.7(4)$ \\
\hline$C(92)-C(72)-C(82)$ & $110.7(4)$ & $\mathrm{C}(33)-\mathrm{C}(23)-\mathrm{C}(13)$ & $117.8(4)$ \\
\hline $\mathrm{C}(22)-\mathrm{C}(72)-\mathrm{H}(72 \mathrm{~A})$ & 107.6 & $\mathrm{C}(33)-\mathrm{C}(23)-\mathrm{C}(73)$ & $119.5(4)$ \\
\hline $\mathrm{C}(92)-\mathrm{C}(72)-\mathrm{H}(72 \mathrm{~A})$ & 107.6 & $\mathrm{C}(13)-\mathrm{C}(23)-\mathrm{C}(73)$ & $122.7(4)$ \\
\hline $\mathrm{C}(82)-\mathrm{C}(72)-\mathrm{H}(72 \mathrm{~A})$ & 107.6 & $\mathrm{C}(43)-\mathrm{C}(33)-\mathrm{C}(23)$ & $121.6(4)$ \\
\hline $\mathrm{C}(72)-\mathrm{C}(82)-\mathrm{H}(82 \mathrm{~A})$ & 109.5 & $\mathrm{C}(43)-\mathrm{C}(33)-\mathrm{H}(33 \mathrm{~A})$ & 119.2 \\
\hline
\end{tabular}




\begin{tabular}{|c|c|c|c|}
\hline $\mathrm{C}(23)-\mathrm{C}(33)-\mathrm{H}(33 \mathrm{~A})$ & 119.2 & $\mathrm{C}(103)-\mathrm{C}(113)-\mathrm{H}(11 \mathrm{I})$ & 109.5 \\
\hline$C(53)-C(43)-C(33)$ & $119.5(4)$ & $\mathrm{H}(11 \mathrm{G})-\mathrm{C}(113)-\mathrm{H}(11 \mathrm{I})$ & 109.5 \\
\hline $\mathrm{C}(53)-\mathrm{C}(43)-\mathrm{H}(43 \mathrm{~A})$ & 120.3 & $\mathrm{H}(11 \mathrm{H})-\mathrm{C}(113)-\mathrm{H}(11 \mathrm{I})$ & 109.5 \\
\hline $\mathrm{C}(33)-\mathrm{C}(43)-\mathrm{H}(43 \mathrm{~A})$ & 120.3 & $\mathrm{C}(103)-\mathrm{C}(123)-\mathrm{H}(12 \mathrm{D})$ & 109.5 \\
\hline $\mathrm{C}(43)-\mathrm{C}(53)-\mathrm{C}(63)$ & $121.5(5)$ & $\mathrm{C}(103)-\mathrm{C}(123)-\mathrm{H}(12 \mathrm{E})$ & 109.5 \\
\hline $\mathrm{C}(43)-\mathrm{C}(53)-\mathrm{H}(53 \mathrm{~A})$ & 119.3 & $\mathrm{H}(12 \mathrm{D})-\mathrm{C}(123)-\mathrm{H}(12 \mathrm{E})$ & 109.5 \\
\hline $\mathrm{C}(63)-\mathrm{C}(53)-\mathrm{H}(53 \mathrm{~A})$ & 119.3 & $\mathrm{C}(103)-\mathrm{C}(123)-\mathrm{H}(12 \mathrm{~F})$ & 109.5 \\
\hline$C(53)-C(63)-C(13)$ & $118.5(4)$ & $\mathrm{H}(12 \mathrm{D})-\mathrm{C}(123)-\mathrm{H}(12 \mathrm{~F})$ & 109.5 \\
\hline$C(53)-C(63)-C(103)$ & $120.0(4)$ & $\mathrm{H}(12 \mathrm{E})-\mathrm{C}(123)-\mathrm{H}(12 \mathrm{~F})$ & 109.5 \\
\hline$C(13)-C(63)-C(103)$ & $121.5(4)$ & $\mathrm{O}(14)-\mathrm{C}(14)-\mathrm{C}(84)$ & $117.6(4)$ \\
\hline$C(23)-C(73)-C(93)$ & $111.0(4)$ & $\mathrm{O}(14)-\mathrm{C}(14)-\mathrm{C}(24)$ & $115.5(4)$ \\
\hline$C(23)-C(73)-C(83)$ & $111.7(4)$ & $C(84)-C(14)-C(24)$ & $119.8(4)$ \\
\hline $\mathrm{C}(93)-\mathrm{C}(73)-\mathrm{C}(83)$ & $109.3(4)$ & $\mathrm{O}(14)-\mathrm{C}(14)-\mathrm{Fe}(1)$ & $60.5(2)$ \\
\hline $\mathrm{C}(23)-\mathrm{C}(73)-\mathrm{H}(73 \mathrm{~A})$ & 108.2 & $\mathrm{C}(84)-\mathrm{C}(14)-\mathrm{Fe}(1)$ & $111.8(3)$ \\
\hline $\mathrm{C}(93)-\mathrm{C}(73)-\mathrm{H}(73 \mathrm{~A})$ & 108.2 & $\mathrm{C}(24)-\mathrm{C}(14)-\mathrm{Fe}(1)$ & $117.8(3)$ \\
\hline $\mathrm{C}(83)-\mathrm{C}(73)-\mathrm{H}(73 \mathrm{~A})$ & 108.2 & $C(34)-C(24)-C(74)$ & $117.6(4)$ \\
\hline $\mathrm{C}(73)-\mathrm{C}(83)-\mathrm{H}(83 \mathrm{~A})$ & 109.5 & $C(34)-C(24)-C(14)$ & $121.7(4)$ \\
\hline $\mathrm{C}(73)-\mathrm{C}(83)-\mathrm{H}(83 \mathrm{~B})$ & 109.5 & $C(74)-C(24)-C(14)$ & $120.5(4)$ \\
\hline $\mathrm{H}(83 \mathrm{~A})-\mathrm{C}(83)-\mathrm{H}(83 \mathrm{~B})$ & 109.5 & $\mathrm{C}(44)-\mathrm{C}(34)-\mathrm{C}(24)$ & $121.6(4)$ \\
\hline $\mathrm{C}(73)-\mathrm{C}(83)-\mathrm{H}(83 \mathrm{C})$ & 109.5 & $\mathrm{C}(44)-\mathrm{C}(34)-\mathrm{H}(34 \mathrm{~A})$ & 119.2 \\
\hline $\mathrm{H}(83 \mathrm{~A})-\mathrm{C}(83)-\mathrm{H}(83 \mathrm{C})$ & 109.5 & $\mathrm{C}(24)-\mathrm{C}(34)-\mathrm{H}(34 \mathrm{~A})$ & 119.2 \\
\hline $\mathrm{H}(83 \mathrm{~B})-\mathrm{C}(83)-\mathrm{H}(83 \mathrm{C})$ & 109.5 & $C(34)-C(44)-C(54)$ & $119.7(5)$ \\
\hline $\mathrm{C}(73)-\mathrm{C}(93)-\mathrm{H}(93 \mathrm{~A})$ & 109.5 & $\mathrm{C}(34)-\mathrm{C}(44)-\mathrm{H}(44 \mathrm{~A})$ & 120.2 \\
\hline $\mathrm{C}(73)-\mathrm{C}(93)-\mathrm{H}(93 \mathrm{~B})$ & 109.5 & $\mathrm{C}(54)-\mathrm{C}(44)-\mathrm{H}(44 \mathrm{~A})$ & 120.2 \\
\hline $\mathrm{H}(93 \mathrm{~A})-\mathrm{C}(93)-\mathrm{H}(93 \mathrm{~B})$ & 109.5 & $C(64)-C(54)-C(44)$ & $119.7(5)$ \\
\hline $\mathrm{C}(73)-\mathrm{C}(93)-\mathrm{H}(93 \mathrm{C})$ & 109.5 & $\mathrm{C}(64)-\mathrm{C}(54)-\mathrm{H}(54 \mathrm{~A})$ & 120.1 \\
\hline $\mathrm{H}(93 \mathrm{~A})-\mathrm{C}(93)-\mathrm{H}(93 \mathrm{C})$ & 109.5 & $\mathrm{C}(44)-\mathrm{C}(54)-\mathrm{H}(54 \mathrm{~A})$ & 120.1 \\
\hline $\mathrm{H}(93 \mathrm{~B})-\mathrm{C}(93)-\mathrm{H}(93 \mathrm{C})$ & 109.5 & $C(54)-C(64)-C(74)$ & $120.8(4)$ \\
\hline$C(113)-C(103)-C(63)$ & $110.0(4)$ & $\mathrm{C}(54)-\mathrm{C}(64)-\mathrm{H}(64 \mathrm{~A})$ & 119.6 \\
\hline$C(113)-C(103)-C(123)$ & $110.3(4)$ & $\mathrm{C}(74)-\mathrm{C}(64)-\mathrm{H}(64 \mathrm{~A})$ & 119.6 \\
\hline$C(63)-C(103)-C(123)$ & $112.5(4)$ & $C(64)-C(74)-C(24)$ & $120.6(5)$ \\
\hline $\mathrm{C}(113)-\mathrm{C}(103)-\mathrm{H}(10 \mathrm{~B})$ & 108.0 & $\mathrm{C}(64)-\mathrm{C}(74)-\mathrm{H}(74 \mathrm{~A})$ & 119.7 \\
\hline $\mathrm{C}(63)-\mathrm{C}(103)-\mathrm{H}(10 \mathrm{~B})$ & 108.0 & $\mathrm{C}(24)-\mathrm{C}(74)-\mathrm{H}(74 \mathrm{~A})$ & 119.7 \\
\hline $\mathrm{C}(123)-\mathrm{C}(103)-\mathrm{H}(10 \mathrm{~B})$ & 108.0 & $\mathrm{C}(94)-\mathrm{C}(84)-\mathrm{C}(134)$ & $118.4(4)$ \\
\hline $\mathrm{C}(103)-\mathrm{C}(113)-\mathrm{H}(11 \mathrm{G})$ & 109.5 & $\mathrm{C}(94)-\mathrm{C}(84)-\mathrm{C}(14)$ & $122.0(4)$ \\
\hline $\mathrm{C}(103)-\mathrm{C}(113)-\mathrm{H}(11 \mathrm{H})$ & 109.5 & $\mathrm{C}(134)-\mathrm{C}(84)-\mathrm{C}(14)$ & $119.6(4)$ \\
\hline $\mathrm{H}(11 \mathrm{G})-\mathrm{C}(113)-\mathrm{H}(11 \mathrm{H})$ & 109.5 & $\mathrm{C}(104)-\mathrm{C}(94)-\mathrm{C}(84)$ & $120.8(4)$ \\
\hline
\end{tabular}




\begin{tabular}{|c|c|c|c|}
\hline $\mathrm{C}(104)-\mathrm{C}(94)-\mathrm{H}(94 \mathrm{~A})$ & 119.6 & $\mathrm{H}(15 \mathrm{E})-\mathrm{C}\left(15^{\prime}\right)-\mathrm{H}(15 \mathrm{~F})$ & 109.5 \\
\hline $\mathrm{C}(84)-\mathrm{C}(94)-\mathrm{H}(94 \mathrm{~A})$ & 119.6 & $\mathrm{C}\left(35^{\prime}\right)-\mathrm{C}\left(25^{\prime}\right)-\mathrm{C}\left(15^{\prime}\right)$ & $109.9(19)$ \\
\hline C(94)-C(104)-C(114) & $120.6(5)$ & $\mathrm{C}\left(35^{\prime}\right)-\mathrm{C}\left(25^{\prime}\right)-\mathrm{H}(25 \mathrm{C})$ & 109.7 \\
\hline $\mathrm{C}(94)-\mathrm{C}(104)-\mathrm{H}(10 \mathrm{C})$ & 119.7 & $\mathrm{C}\left(15^{\prime}\right)-\mathrm{C}\left(25^{\prime}\right)-\mathrm{H}(25 \mathrm{C})$ & 109.7 \\
\hline $\mathrm{C}(114)-\mathrm{C}(104)-\mathrm{H}(10 \mathrm{C})$ & 119.7 & $\mathrm{C}\left(35^{\prime}\right)-\mathrm{C}\left(25^{\prime}\right)-\mathrm{H}(25 \mathrm{D})$ & 109.7 \\
\hline $\mathrm{C}(124)-\mathrm{C}(114)-\mathrm{C}(104)$ & $119.4(5)$ & $\mathrm{C}\left(15^{\prime}\right)-\mathrm{C}\left(25^{\prime}\right)-\mathrm{H}(25 \mathrm{D})$ & 109.7 \\
\hline $\mathrm{C}(124)-\mathrm{C}(114)-\mathrm{H}(11 \mathrm{~J})$ & 120.3 & $\mathrm{H}(25 \mathrm{C})-\mathrm{C}\left(25^{\prime}\right)-\mathrm{H}(25 \mathrm{D})$ & 108.2 \\
\hline $\mathrm{C}(104)-\mathrm{C}(114)-\mathrm{H}(11 \mathrm{~J})$ & 120.3 & $\mathrm{C}\left(35^{\prime}\right) \# 1-\mathrm{C}\left(35^{\prime}\right)-\mathrm{C}\left(25^{\prime}\right)$ & $110.9(19)$ \\
\hline $\mathrm{C}(114)-\mathrm{C}(124)-\mathrm{C}(134)$ & $121.1(5)$ & $\mathrm{C}\left(35^{\prime}\right) \# 1-\mathrm{C}\left(35^{\prime}\right)-\mathrm{H}(35 \mathrm{C})$ & 109.5 \\
\hline $\mathrm{C}(114)-\mathrm{C}(124)-\mathrm{H}(12 \mathrm{G})$ & 119.5 & $\mathrm{C}\left(25^{\prime}\right)-\mathrm{C}\left(35^{\prime}\right)-\mathrm{H}(35 \mathrm{C})$ & 109.5 \\
\hline $\mathrm{C}(134)-\mathrm{C}(124)-\mathrm{H}(12 \mathrm{G})$ & 119.5 & $\mathrm{C}\left(35^{\prime}\right) \# 1-\mathrm{C}\left(35^{\prime}\right)-\mathrm{H}(35 \mathrm{D})$ & 109.5 \\
\hline $\mathrm{C}(124)-\mathrm{C}(134)-\mathrm{C}(84)$ & $119.7(5)$ & $\mathrm{C}\left(25^{\prime}\right)-\mathrm{C}\left(35^{\prime}\right)-\mathrm{H}(35 \mathrm{D})$ & 109.5 \\
\hline $\mathrm{C}(124)-\mathrm{C}(134)-\mathrm{H}(13 \mathrm{~A})$ & 120.2 & $\mathrm{H}(35 \mathrm{C})-\mathrm{C}\left(35^{\prime}\right)-\mathrm{H}(35 \mathrm{D})$ & 108.1 \\
\hline $\mathrm{C}(84)-\mathrm{C}(134)-\mathrm{H}(13 \mathrm{~A})$ & 120.2 & & \\
\hline $\mathrm{C}(25)-\mathrm{C}(15)-\mathrm{H}(15 \mathrm{~A})$ & 109.5 & & \\
\hline $\mathrm{C}(25)-\mathrm{C}(15)-\mathrm{H}(15 \mathrm{~B})$ & 109.5 & & \\
\hline $\mathrm{H}(15 \mathrm{~A})-\mathrm{C}(15)-\mathrm{H}(15 \mathrm{~B})$ & 109.5 & & \\
\hline $\mathrm{C}(25)-\mathrm{C}(15)-\mathrm{H}(15 \mathrm{C})$ & 109.5 & & \\
\hline $\mathrm{H}(15 \mathrm{~A})-\mathrm{C}(15)-\mathrm{H}(15 \mathrm{C})$ & 109.5 & & \\
\hline $\mathrm{H}(15 \mathrm{~B})-\mathrm{C}(15)-\mathrm{H}(15 \mathrm{C})$ & 109.5 & & \\
\hline $\mathrm{C}(35)-\mathrm{C}(25)-\mathrm{C}(15)$ & $112.1(8)$ & & \\
\hline $\mathrm{C}(35)-\mathrm{C}(25)-\mathrm{H}(25 \mathrm{~A})$ & 109.2 & & \\
\hline $\mathrm{C}(15)-\mathrm{C}(25)-\mathrm{H}(25 \mathrm{~A})$ & 109.2 & & \\
\hline $\mathrm{C}(35)-\mathrm{C}(25)-\mathrm{H}(25 \mathrm{~B})$ & 109.2 & & \\
\hline $\mathrm{C}(15)-\mathrm{C}(25)-\mathrm{H}(25 \mathrm{~B})$ & 109.2 & & \\
\hline $\mathrm{H}(25 \mathrm{~A})-\mathrm{C}(25)-\mathrm{H}(25 \mathrm{~B})$ & 107.9 & & \\
\hline $\mathrm{C}(35) \# 1-\mathrm{C}(35)-\mathrm{C}(25)$ & $113.0(9)$ & & \\
\hline $\mathrm{C}(35) \# 1-\mathrm{C}(35)-\mathrm{H}(35 \mathrm{~A})$ & 109.0 & & \\
\hline $\mathrm{C}(25)-\mathrm{C}(35)-\mathrm{H}(35 \mathrm{~A})$ & 109.0 & & \\
\hline $\mathrm{C}(35) \# 1-\mathrm{C}(35)-\mathrm{H}(35 \mathrm{~B})$ & 109.0 & & \\
\hline $\mathrm{C}(25)-\mathrm{C}(35)-\mathrm{H}(35 \mathrm{~B})$ & 109.0 & & \\
\hline $\mathrm{H}(35 \mathrm{~A})-\mathrm{C}(35)-\mathrm{H}(35 \mathrm{~B})$ & 107.8 & & \\
\hline $\mathrm{C}\left(25^{\prime}\right)-\mathrm{C}\left(15^{\prime}\right)-\mathrm{H}(15 \mathrm{D})$ & 109.5 & & \\
\hline $\mathrm{C}\left(25^{\prime}\right)-\mathrm{C}\left(15^{\prime}\right)-\mathrm{H}(15 \mathrm{E})$ & 109.5 & & \\
\hline $\mathrm{H}(15 \mathrm{D})-\mathrm{C}\left(15^{\prime}\right)-\mathrm{H}(15 \mathrm{E})$ & 109.5 & & \\
\hline $\mathrm{C}\left(25^{\prime}\right)-\mathrm{C}\left(15^{\prime}\right)-\mathrm{H}(15 \mathrm{~F})$ & 109.5 & & \\
\hline $\mathrm{H}(15 \mathrm{D})-\mathrm{C}\left(15^{\prime}\right)-\mathrm{H}(15 \mathrm{~F})$ & 109.5 & & \\
\hline
\end{tabular}


Symmetry transformations used to generate equivalent atoms:

$\# 1-x+1,-y+1,-z$ 
Table S-10. Anisotropic displacement parameters $\left(\AA^{2} \times 10^{3}\right)$ for 2 . The anisotropic displacement factor exponent takes the form: $-2 \pi^{2}\left[\mathrm{~h}^{2} \mathrm{a}^{* 2} \mathrm{U}_{11}+\ldots+2 \mathrm{~h} \mathrm{k} \mathrm{a}^{*} \mathrm{~b}^{*} \mathrm{U}_{12}\right]$

\begin{tabular}{|c|c|c|c|c|c|c|}
\hline & $\mathrm{U}_{11}$ & $\mathrm{U}_{22}$ & $\mathrm{U}_{33}$ & $\mathrm{U}_{23}$ & $\mathrm{U}_{13}$ & $\mathrm{U}_{12}$ \\
\hline $\mathrm{Fe} 1$ & $25(1)$ & $15(1)$ & $13(1)$ & $1(1)$ & $7(1)$ & $1(1)$ \\
\hline O14 & $33(2)$ & $17(2)$ & $15(2)$ & $0(1)$ & $11(2)$ & $2(1)$ \\
\hline N11 & $19(2)$ & $17(2)$ & $11(2)$ & $0(2)$ & $4(2)$ & $-2(2)$ \\
\hline $\mathrm{N} 21$ & $20(2)$ & $14(2)$ & $18(2)$ & $-1(2)$ & $8(2)$ & $4(2)$ \\
\hline C11 & $40(3)$ & $22(3)$ & $16(2)$ & $5(2)$ & $12(2)$ & $7(2)$ \\
\hline $\mathrm{C} 21$ & $30(3)$ & $21(3)$ & $14(2)$ & $1(2)$ & $4(2)$ & $4(2)$ \\
\hline $\mathrm{C} 31$ & $30(3)$ & $17(3)$ & $12(2)$ & $3(2)$ & $5(2)$ & $0(2)$ \\
\hline $\mathrm{C} 41$ & $20(3)$ & $18(3)$ & $11(2)$ & $-3(2)$ & $3(2)$ & $-5(2)$ \\
\hline C51 & $32(3)$ & $22(3)$ & $21(3)$ & $2(2)$ & $11(2)$ & $1(2)$ \\
\hline $\mathrm{C} 12$ & $30(3)$ & $16(3)$ & $10(2)$ & $3(2)$ & $8(2)$ & $0(2)$ \\
\hline $\mathrm{C} 22$ & $33(3)$ & $14(3)$ & $15(2)$ & $2(2)$ & $6(2)$ & $0(2)$ \\
\hline $\mathrm{C} 32$ & $30(3)$ & $21(3)$ & $13(2)$ & $0(2)$ & $5(2)$ & $2(2)$ \\
\hline $\mathrm{C} 42$ & $41(3)$ & $20(3)$ & $15(2)$ & $-3(2)$ & $10(2)$ & $4(2)$ \\
\hline C52 & $36(3)$ & $20(3)$ & $24(3)$ & $2(2)$ & $17(2)$ & $1(2)$ \\
\hline C62 & $30(3)$ & $16(3)$ & $19(2)$ & $5(2)$ & $6(2)$ & $5(2)$ \\
\hline C72 & $27(3)$ & $23(3)$ & $17(2)$ & $-1(2)$ & $3(2)$ & $-3(2)$ \\
\hline C82 & $29(3)$ & $30(3)$ & $28(3)$ & $0(2)$ & $5(3)$ & $-1(2)$ \\
\hline C92 & $39(3)$ & $23(3)$ & $39(3)$ & $-2(2)$ & $15(3)$ & $-6(2)$ \\
\hline $\mathrm{C} 102$ & $27(3)$ & $23(3)$ & $20(3)$ & $-4(2)$ & $10(2)$ & $1(2)$ \\
\hline C112 & $35(3)$ & $31(3)$ & $34(3)$ & $-8(2)$ & $10(3)$ & $-12(3)$ \\
\hline $\mathrm{C} 122$ & $47(4)$ & $39(3)$ & $31(3)$ & $4(3)$ & $-4(3)$ & $-4(3)$ \\
\hline $\mathrm{C} 13$ & $26(3)$ & $22(3)$ & $11(2)$ & $3(2)$ & $10(2)$ & $2(2)$ \\
\hline $\mathrm{C} 23$ & $24(3)$ & 21(3) & $15(2)$ & $3(2)$ & $8(2)$ & $0(2)$ \\
\hline $\mathrm{C} 33$ & $35(3)$ & $17(3)$ & $16(2)$ & $-2(2)$ & $9(2)$ & $-1(2)$ \\
\hline $\mathrm{C} 43$ & $28(3)$ & $21(3)$ & $21(2)$ & $2(2)$ & $10(2)$ & $4(2)$ \\
\hline C53 & $18(3)$ & $30(3)$ & $19(3)$ & $-1(2)$ & $6(2)$ & $2(2)$ \\
\hline C63 & $24(3)$ & $18(3)$ & $14(2)$ & $3(2)$ & $11(2)$ & $5(2)$ \\
\hline $\mathrm{C} 73$ & $26(3)$ & $17(3)$ & $19(2)$ & $-4(2)$ & $8(2)$ & $-1(2)$ \\
\hline $\mathrm{C} 83$ & $34(3)$ & $23(3)$ & $22(3)$ & $-2(2)$ & $9(2)$ & $-7(2)$ \\
\hline C93 & $29(3)$ & $42(3)$ & $27(3)$ & $3(2)$ & $8(3)$ & $-6(3)$ \\
\hline $\mathrm{C} 103$ & $23(3)$ & 19(3) & $24(3)$ & $1(2)$ & $7(2)$ & $-2(2)$ \\
\hline $\mathrm{C} 113$ & $51(4)$ & $33(3)$ & $29(3)$ & $-6(2)$ & $6(3)$ & $-15(3)$ \\
\hline
\end{tabular}




\begin{tabular}{lllcccc} 
C123 & $35(3)$ & $23(3)$ & $57(4)$ & $-6(3)$ & $23(3)$ & $-3(3)$ \\
C14 & $34(3)$ & $18(3)$ & $9(2)$ & $0(2)$ & $10(2)$ & $-2(2)$ \\
C24 & $24(3)$ & $17(3)$ & $15(2)$ & $-1(2)$ & $10(2)$ & $1(2)$ \\
C34 & $29(3)$ & $15(3)$ & $24(3)$ & $4(2)$ & $9(2)$ & $2(2)$ \\
C44 & $24(3)$ & $32(3)$ & $30(3)$ & $11(2)$ & $4(2)$ & $11(2)$ \\
C54 & $27(3)$ & $27(3)$ & $31(3)$ & $-2(2)$ & $10(3)$ & $-5(2)$ \\
C64 & $39(3)$ & $19(3)$ & $17(2)$ & $1(2)$ & $10(2)$ & $-1(2)$ \\
C74 & $28(3)$ & $18(3)$ & $18(2)$ & $1(2)$ & $10(2)$ & $1(2)$ \\
C84 & $27(3)$ & $15(2)$ & $12(2)$ & $5(2)$ & $7(2)$ & $-3(2)$ \\
C94 & $27(3)$ & $12(2)$ & $19(2)$ & $3(2)$ & $8(2)$ & $-1(2)$ \\
C104 & $27(3)$ & $19(3)$ & $33(3)$ & $5(2)$ & $18(3)$ & $5(2)$ \\
C114 & $20(3)$ & $36(3)$ & $25(3)$ & $6(2)$ & $4(2)$ & $1(2)$ \\
C124 & $29(3)$ & $27(3)$ & $20(3)$ & $4(2)$ & $3(2)$ & $-7(2)$ \\
C134 & $28(3)$ & $16(3)$ & $22(3)$ & $1(2)$ & $10(2)$ & $-4(2)$ \\
C15 & $78(9)$ & $61(6)$ & $69(13)$ & $-12(11)$ & $39(9)$ & $-15(7)$ \\
C25 & $62(6)$ & $31(5)$ & $43(5)$ & $9(3)$ & $20(5)$ & $-2(4)$ \\
C35 & $55(6)$ & $31(4)$ & $38(6)$ & $5(5)$ & $14(5)$ & $0(4)$ \\
C15' & $78(9)$ & $61(6)$ & $69(13)$ & $-12(11)$ & $39(9)$ & $-15(7)$ \\
C25' & $62(6)$ & $31(5)$ & $43(5)$ & $9(3)$ & $20(5)$ & $-2(4)$ \\
C35' & $55(6)$ & $31(4)$ & $38(6)$ & $5(5)$ & $14(5)$ & $0(4)$ \\
& & & & & & \\
\hline & & & & & &
\end{tabular}


Table S-11. Hydrogen coordinates $\left(\times 10^{4}\right)$ and isotropic displacement parameters $\left(\AA^{2} \times 10^{3}\right)$ for $\left[\mathrm{L}^{\mathrm{Me}} \mathrm{Fe}\left(\mathrm{Ph}_{2} \mathrm{CO}\right]\right.$ (2).

\begin{tabular}{|c|c|c|c|c|}
\hline & $\mathrm{x}$ & $\mathrm{y}$ & $\mathrm{z}$ & $\mathrm{U}(\mathrm{eq})$ \\
\hline $\mathrm{H} 11 \mathrm{~A}$ & 3064 & 4957 & 698 & 38 \\
\hline H11B & 3251 & 5234 & 40 & 38 \\
\hline $\mathrm{H} 11 \mathrm{C}$ & 3471 & 5344 & 944 & 38 \\
\hline $\mathrm{H} 31 \mathrm{~A}$ & 3450 & 3781 & -497 & 24 \\
\hline $\mathrm{H} 51 \mathrm{~A}$ & 3696 & 1382 & -1179 & 37 \\
\hline H51B & 4032 & 2177 & -759 & 37 \\
\hline $\mathrm{H} 51 \mathrm{C}$ & 3671 & 2784 & -1258 & 37 \\
\hline $\mathrm{H} 32 \mathrm{~A}$ & 3666 & 4810 & 3491 & 26 \\
\hline $\mathrm{H} 42 \mathrm{~A}$ & 3095 & 4733 & 3550 & 30 \\
\hline $\mathrm{H} 52 \mathrm{~A}$ & 2657 & 3797 & 2573 & 30 \\
\hline $\mathrm{H} 72 \mathrm{~A}$ & 3967 & 3835 & 1892 & 28 \\
\hline $\mathrm{H} 82 \mathrm{~A}$ & 4099 & 2413 & 2884 & 45 \\
\hline H82B & 4185 & 3417 & 3539 & 45 \\
\hline $\mathrm{H} 82 \mathrm{C}$ & 4435 & 3251 & 2989 & 45 \\
\hline $\mathrm{H} 92 \mathrm{~A}$ & 3973 & 5847 & 2164 & 50 \\
\hline H92B & 4359 & 5313 & 2561 & 50 \\
\hline $\mathrm{H} 92 \mathrm{C}$ & 4103 & 5591 & 3083 & 50 \\
\hline $\mathrm{H} 10 \mathrm{~A}$ & 2786 & 2343 & 902 & 27 \\
\hline H11D & 2661 & 1295 & 1939 & 50 \\
\hline $\mathrm{H} 11 \mathrm{E}$ & 2321 & 1394 & 1189 & 50 \\
\hline $\mathrm{H} 11 \mathrm{~F}$ & 2357 & 2226 & 1929 & 50 \\
\hline $\mathrm{H} 12 \mathrm{~A}$ & 2518 & 4145 & 484 & 64 \\
\hline H12B & 2310 & 4115 & 1126 & 64 \\
\hline $\mathrm{H} 12 \mathrm{C}$ & 2209 & 3206 & 412 & 64 \\
\hline $\mathrm{H} 33 \mathrm{~A}$ & 3885 & -2346 & -496 & 27 \\
\hline $\mathrm{H} 43 \mathrm{~A}$ & 4498 & -2152 & -84 & 27 \\
\hline $\mathrm{H} 53 \mathrm{~A}$ & 4755 & -418 & 504 & 27 \\
\hline $\mathrm{H} 73 \mathrm{~A}$ & 3265 & -288 & -165 & 25 \\
\hline $\mathrm{H} 83 \mathrm{~A}$ & 3402 & -2275 & 358 & 39 \\
\hline H83B & 3325 & -2717 & -524 & 39 \\
\hline $\mathrm{H} 83 \mathrm{C}$ & 3015 & -2126 & -247 & 39 \\
\hline
\end{tabular}




\begin{tabular}{|c|c|c|c|c|}
\hline H93A & 3262 & 58 & -1447 & 49 \\
\hline H93B & 2970 & -924 & -1465 & 49 \\
\hline H93C & 3344 & -1297 & -1566 & 49 \\
\hline H10B & 4299 & 2230 & 673 & 26 \\
\hline $\mathrm{H} 11 \mathrm{G}$ & 4362 & 1299 & 1863 & 59 \\
\hline $\mathrm{H} 11 \mathrm{H}$ & 4692 & 2158 & 1957 & 59 \\
\hline H11I & 4737 & 771 & 1868 & 59 \\
\hline $\mathrm{H} 12 \mathrm{D}$ & 4734 & 1948 & 6 & 54 \\
\hline $\mathrm{H} 12 \mathrm{E}$ & 4986 & 1326 & 771 & 54 \\
\hline $\mathrm{H} 12 \mathrm{~F}$ & 4885 & 2687 & 794 & 54 \\
\hline $\mathrm{H} 34 \mathrm{~A}$ & 2911 & 89 & 1038 & 27 \\
\hline $\mathrm{H} 44 \mathrm{~A}$ & 2448 & -1249 & 685 & 36 \\
\hline $\mathrm{H} 54 \mathrm{~A}$ & 2535 & -3185 & 1149 & 34 \\
\hline H64A & 3086 & -3765 & 1945 & 29 \\
\hline $\mathrm{H} 74 \mathrm{~A}$ & 3554 & -2450 & 2282 & 25 \\
\hline H94A & 3975 & -1684 & 1421 & 23 \\
\hline $\mathrm{H} 10 \mathrm{C}$ & 4547 & -2332 & 2009 & 29 \\
\hline $\mathrm{H} 11 \mathrm{~J}$ & 4863 & -1670 & 3252 & 33 \\
\hline $\mathrm{H} 12 \mathrm{G}$ & 4609 & -301 & 3883 & 32 \\
\hline $\mathrm{H} 13 \mathrm{~A}$ & 4040 & 398 & 3300 & 26 \\
\hline $\mathrm{H} 15 \mathrm{~A}$ & 4513 & 4717 & 1264 & 98 \\
\hline H15B & 4132 & 5009 & 670 & 98 \\
\hline $\mathrm{H} 15 \mathrm{C}$ & 4419 & 6033 & 955 & 98 \\
\hline $\mathrm{H} 25 \mathrm{~A}$ & 4476 & 4115 & -42 & 53 \\
\hline $\mathrm{H} 25 \mathrm{~B}$ & 4404 & 5454 & -322 & 53 \\
\hline $\mathrm{H} 35 \mathrm{~A}$ & 4974 & 5998 & 462 & 49 \\
\hline H35B & 5047 & 4686 & 794 & 49 \\
\hline H15D & 4178 & 5541 & 42 & 98 \\
\hline $\mathrm{H} 15 \mathrm{E}$ & 4242 & 5609 & 965 & 98 \\
\hline $\mathrm{H} 15 \mathrm{~F}$ & 4256 & 4366 & 556 & 98 \\
\hline $\mathrm{H} 25 \mathrm{C}$ & 4779 & 6195 & 821 & 53 \\
\hline $\mathrm{H} 25 \mathrm{D}$ & 4846 & 4856 & 1108 & 53 \\
\hline $\mathrm{H} 35 \mathrm{C}$ & 4689 & 4298 & -286 & 49 \\
\hline $\mathrm{H} 35 \mathrm{D}$ & 4710 & 5668 & -476 & 49 \\
\hline
\end{tabular}


Table S-12. Torsion angles $\left[{ }^{\circ}\right]$ for $\mathbf{2}$.

\begin{tabular}{|c|c|}
\hline N21-Fe1-O14-C14 & $-16.9(6)$ \\
\hline N11-Fe1-O14-C14 & $173.1(3)$ \\
\hline C12-N11-C21-C31 & $176.0(4)$ \\
\hline Fe1-N11-C21-C31 & $0.5(6)$ \\
\hline C12-N11-C21-C11 & $-1.5(6)$ \\
\hline Fe1-N11-C21-C11 & $-177.0(3)$ \\
\hline N11-C21-C31-C41 & $-7.4(8)$ \\
\hline C11-C21-C31-C41 & $170.1(5)$ \\
\hline C13-N21-C41-C31 & $-169.6(4)$ \\
\hline Fe1-N21-C41-C31 & $3.4(6)$ \\
\hline C13-N21-C41-C51 & $7.2(6)$ \\
\hline Fe1-N21-C41-C51 & $-179.7(3)$ \\
\hline C21-C31-C41-N21 & $5.3(8)$ \\
\hline C21-C31-C41-C51 & $-171.7(5)$ \\
\hline C21-N11-C12-C62 & $95.2(5)$ \\
\hline Fe1-N11-C12-C62 & $-89.1(4)$ \\
\hline C21-N11-C12-C22 & $-87.8(5)$ \\
\hline Fe1-N11-C12-C22 & $87.9(4)$ \\
\hline C62-C12-C22-C32 & $-0.9(6)$ \\
\hline N11-C12-C22-C32 & $-177.9(4)$ \\
\hline C62-C12-C22-C72 & $177.1(4)$ \\
\hline N11-C12-C22-C72 & $0.1(6)$ \\
\hline $\mathrm{C} 12-\mathrm{C} 22-\mathrm{C} 32-\mathrm{C} 42$ & $-0.9(7)$ \\
\hline C72-C22-C32-C42 & $-179.0(4)$ \\
\hline C22-C32-C42-C52 & $2.2(7)$ \\
\hline C32-C42-C52-C62 & $-1.7(7)$ \\
\hline C22-C12-C62-C52 & $1.4(7)$ \\
\hline N11-C12-C62-C52 & $178.3(4)$ \\
\hline C22-C12-C62-C102 & $-176.8(4)$ \\
\hline N11-C12-C62-C102 & $0.1(6)$ \\
\hline C42-C52-C62-C12 & $-0.1(7)$ \\
\hline C42-C52-C62-C102 & $178.2(4)$ \\
\hline C32-C22-C72-C92 & $-52.7(6)$ \\
\hline C12-C22-C72-C92 & $129.3(5)$ \\
\hline C32-C22-C72-C82 & $71.6(5)$ \\
\hline C12-C22-C72-C82 & $-106.3(5)$ \\
\hline
\end{tabular}




\begin{tabular}{|c|c|}
\hline C12-C62-C102-C112 & $127.9(5)$ \\
\hline C52-C62-C102-C112 & $-50.3(6)$ \\
\hline C12-C62-C102-C122 & $-108.8(5)$ \\
\hline C52-C62-C102-C122 & $73.0(5)$ \\
\hline C41-N21-C13-C63 & $79.4(5)$ \\
\hline Fe1-N21-C13-C63 & $-93.8(4)$ \\
\hline C41-N21-C13-C23 & $-102.0(5)$ \\
\hline Fe1-N21-C13-C23 & $84.8(4)$ \\
\hline C63-C13-C23-C33 & $-0.8(6)$ \\
\hline N21-C13-C23-C33 & $-179.3(4)$ \\
\hline C63-C13-C23-C73 & 179.6(4) \\
\hline N21-C13-C23-C73 & $1.0(6)$ \\
\hline C13-C23-C33-C43 & $1.2(7)$ \\
\hline C73-C23-C33-C43 & $-179.2(4)$ \\
\hline C23-C33-C43-C53 & $-0.3(7)$ \\
\hline C33-C43-C53-C63 & $-1.1(7)$ \\
\hline C43-C53-C63-C13 & $1.5(7)$ \\
\hline C43-C53-C63-C103 & $178.9(4)$ \\
\hline C23-C13-C63-C53 & $-0.5(6)$ \\
\hline N21-C13-C63-C53 & $178.0(4)$ \\
\hline C23-C13-C63-C103 & $-177.9(4)$ \\
\hline N21-C13-C63-C103 & $0.7(6)$ \\
\hline C33-C23-C73-C93 & $-78.5(5)$ \\
\hline C13-C23-C73-C93 & $101.1(5)$ \\
\hline C33-C23-C73-C83 & $43.7(6)$ \\
\hline C13-C23-C73-C83 & $-136.6(4)$ \\
\hline C53-C63-C103-C113 & $-82.9(6)$ \\
\hline C13-C63-C103-C113 & $94.4(5)$ \\
\hline C53-C63-C103-C123 & $40.5(6)$ \\
\hline C13-C63-C103-C123 & $-142.2(4)$ \\
\hline Fe1-O14-C14-C84 & $100.7(4)$ \\
\hline Fe1-O14-C14-C24 & $-108.9(3)$ \\
\hline O14-C14-C24-C34 & $42.2(6)$ \\
\hline C84-C14-C24-C34 & $-168.1(4)$ \\
\hline Fe1-C14-C24-C34 & $-26.4(6)$ \\
\hline O14-C14-C24-C74 & $-132.6(4)$ \\
\hline C84-C14-C24-C74 & $17.1(6)$ \\
\hline
\end{tabular}




$\begin{array}{lc}\text { Fe1-C14-C24-C74 } & 158.8(3) \\ \text { C74-C24-C34-C44 } & -1.8(7) \\ \text { C14-C24-C34-C44 } & -176.8(4) \\ \text { C24-C34-C44-C54 } & 1.5(7) \\ \text { C34-C44-C54-C64 } & -0.3(8) \\ \text { C44-C54-C64-C74 } & -0.4(7) \\ \text { C54-C64-C74-C24 } & 0.0(7) \\ \text { C34-C24-C74-C64 } & 1.1(7) \\ \text { C14-C24-C74-C64 } & 176.1(4) \\ \text { O14-C14-C84-C94 } & -155.9(4) \\ \text { C24-C14-C84-C94 } & 54.9(6) \\ \text { Fe1-C14-C84-C94 } & -88.8(4) \\ \text { O14-C14-C84-C134 } & 24.1(6) \\ \text { C24-C14-C84-C134 } & -125.0(4) \\ \text { Fe1-C14-C84-C134 } & 91.2(4) \\ \text { C134-C84-C94-C104 } & 0.9(7) \\ \text { C14-C84-C94-C104 } & -179.1(4) \\ \text { C84-C94-C104-C114 } & 0.5(7) \\ \text { C94-C104-C114-C124 } & -1.2(7) \\ \text { C104-C114-C124-C134 } & 0.5(7) \\ \text { C114-C124-C134-C84 } & 0.8(7) \\ \text { C94-C84-C134-C124 } & -1.5(6) \\ \text { C14-C84-C134-C124 } & 178.5(4) \\ \text { C15-C25-C35-C35\#1 } & 176.5(13) \\ \text { C15'-C25'-C35'-C35'\#1 } & -168(4) \\ & \end{array}$

Symmetry transformations used to generate equivalent atoms:

$\# 1-x+1,-y+1,-z$ 
$\left[\mathbf{L}^{\mathrm{Me}} \mathrm{FeCH}_{3}\right][\mathbf{K}(\mathbf{1 8}$-crown-6)] (4). Low-temperature diffraction data ( $\omega$-scans) were collected on a Rigaku R-AXIS RAPID diffractometer coupled to an R-AXIS RAPID imaging plate detector with Mo K $\alpha$ radiation $(\lambda=0.71073 \AA)$ for the structure of 4 . The diffraction images were processed and scaled using the Rigaku CrystalClear software (CrystalClear and CrystalStructure; Rigaku/MSC: The Woodlands, TX, 2005). The structure was solved with SHELXT and was refined against F2 on all data by full-matrix least squares with SHELXL (Sheldrick, G. M. Acta Cryst. 2008, A64, 112-122). All non-hydrogen atoms were refined anisotropically. Hydrogen atoms were included in the model at geometrically calculated positions and refined using a riding model. The isotropic displacement parameters of all hydrogen atoms were fixed to 1.2 times the $\mathrm{U}$ value of the atoms to which they are linked (1.5 times for methyl groups). The $\mathrm{N}$-aryl groups of the diketiminate supporting ligand are disordered. The site occupancies of the disordered groups were freely refined and converged to benearly equal occupiancies. Chemically identical 1,2 C-C distances were restrained to be similar. An attempt was made to model the 18-crown-6 as disordered, but a stable model was not obtained. Some 1,3 C-C distances of the crown ether converged at chemically unreasonable distances, and were subsequently restrained to $2.4(2) \AA$. Many of the geometrically generated solvent atoms caused close contact with those generated by symmetry. The program SQUEEZE was used to compensate for the contribution of disordered solvents contained in voids within the crystal lattice from the diffraction intensities. This procedure was applied to the data file and the submitted model is based on the solvent-removed data. Based on the total electron density found in the voids $\left(198 \mathrm{e} / \AA^{3}\right)$, it is likely that $\sim 4$ toluene molecules are present in the unit cell. See "_platon_squeeze_details" in this .cif for more information. The full numbering scheme of compound $\mathbf{4}$ can be found in the full details of the X-ray structure determination (CIF), which is included as Supporting Information. 


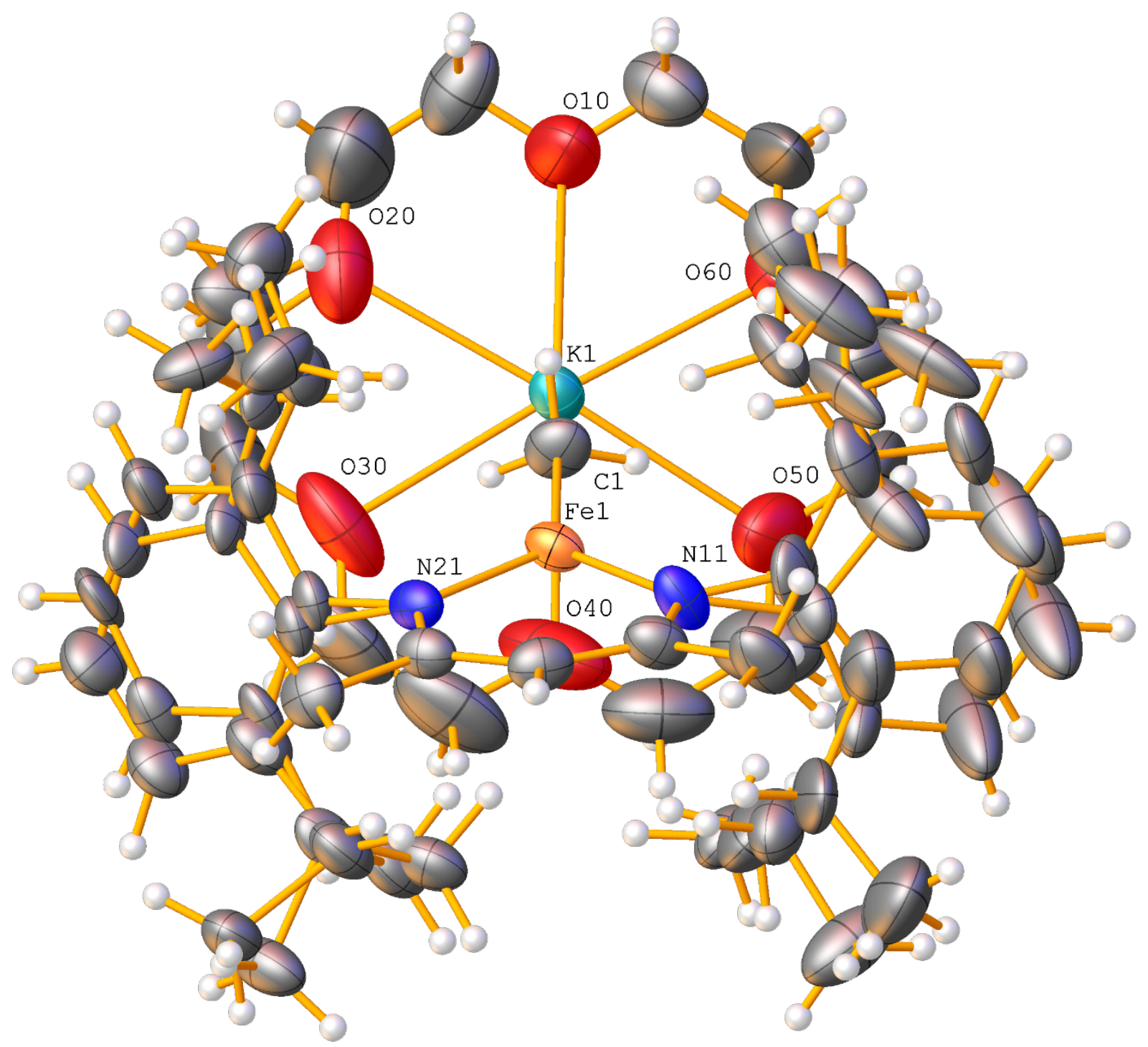

Figure S-26: A partial numbering scheme of 4 with 50\% thermal ellipsoid probability levels. The hydrogen atoms are shown as circles for clarity. 
Table S-13. Crystal data and structure refinement for 4.

Identification code

CSD Deposition Number

Empirical formula

Formula weight

Temperature

Wavelength

Crystal system

Space group

Unit cell dimensions

Volume

Z

Density (calculated)

Absorption coefficient

$\mathrm{F}(000)$

Crystal size

Crystal color and habit

Diffractometer

Theta range for data collection

Index ranges

Reflections collected

Independent reflections

Observed reflections (I > 2sigma(I))

Completeness to theta $=25.028^{\circ}$

Absorption correction

Max. and min. transmission

Solution method

Refinement method

Data / restraints / parameters

Goodness-of-fit on $F^{2}$

Final $R$ indices [I $>2$ sigma(I)]

$R$ indices (all data)

Absolute structure parameter

Largest diff. peak and hole spider-16053

1944810

$\mathrm{C}_{42} \mathrm{H}_{68} \mathrm{FeKN}_{2} \mathrm{O}_{6}$

791.93

93(2) K

$0.71075 \AA$

Orthorhombic

$P 212121$

$a=16.6896(5) \AA$

$\alpha=90^{\circ}$.

$b=24.5737(17) \AA$

$\beta=90^{\circ}$.

$c=12.2392(4) \AA$

$\gamma=90^{\circ}$.

5019.6(4) $\AA^{3}$

4

$1.048 \mathrm{~g} / \mathrm{cm}^{3}$

$0.422 \mathrm{~mm}^{-1}$

1708

$0.250 \times 0.150 \times 0.100 \mathrm{~mm}^{3}$

Red block

Rigaku R-AXIS RAPID imaging plate

2.992 to $25.028^{\circ}$.

$-19<=\mathrm{h}<=19,-29<=\mathrm{k}<=29,-14<=\mathrm{l}<=14$

77966

$8866[\mathrm{R}($ int $)=0.1154]$

7995

$99.7 \%$

Semi-empirical from equivalents

1.000 and 0.663

SHELXT-2014/5 (Sheldrick, 2014)

SHELXL-2014/7 (Sheldrick, 2014)

8866 / $950 / 700$

1.038

$\mathrm{R} 1=0.0868, \mathrm{wR} 2=0.2196$

$\mathrm{R} 1=0.0933, \mathrm{wR} 2=0.2252$

$0.08(5)$

0.858 and -0.371 e $\AA^{-3}$ 
Table S-14. Atomic coordinates ( $\times 10^{4}$ ) and equivalent isotropic displacement parameters $\left(\AA^{2} \times 10^{3}\right)$ for $4 . U(e q)$ is defined as one third of the trace of the orthogonalized $U^{i j}$ tensor.

\begin{tabular}{|c|c|c|c|c|}
\hline & $\mathrm{x}$ & $\mathrm{y}$ & $\mathrm{z}$ & $\mathrm{U}(\mathrm{eq})$ \\
\hline $\mathrm{Fe}(1)$ & $4819(1)$ & $6406(1)$ & $3342(1)$ & $37(1)$ \\
\hline$C(1)$ & $5543(5)$ & 7051(3) & $3062(8)$ & $50(2)$ \\
\hline $\mathrm{N}(11)$ & $4863(4)$ & $5832(3)$ & $4395(5)$ & $43(2)$ \\
\hline $\mathrm{N}(21)$ & $3803(4)$ & $6229(3)$ & $2673(6)$ & $38(2)$ \\
\hline $\mathrm{C}(11)$ & $4251(6)$ & $5116(4)$ & $5577(8)$ & $59(3)$ \\
\hline $\mathrm{C}(21)$ & $4212(5)$ & $5497(3)$ & $4590(7)$ & $44(2)$ \\
\hline $\mathrm{C}(31)$ & $3515(5)$ & $5510(3)$ & $3993(7)$ & $42(2)$ \\
\hline $\mathrm{C}(41)$ & $3318(4)$ & $5832(3)$ & $3059(7)$ & $38(2)$ \\
\hline $\mathrm{C}(51)$ & $2508(5)$ & $5718(4)$ & $2505(8)$ & $49(2)$ \\
\hline$C(1 \mathrm{~A})$ & $5669(13)$ & $5663(11)$ & $4880(20)$ & $43(6)$ \\
\hline $\mathrm{C}(2 \mathrm{~A})$ & $6185(13)$ & $5268(12)$ & $4432(18)$ & $54(5)$ \\
\hline$C(3 \mathrm{~A})$ & $6964(15)$ & $5170(19)$ & $4790(20)$ & $115(13)$ \\
\hline $\mathrm{C}(4 \mathrm{~A})$ & $7224(18)$ & $5461(19)$ & $5720(20)$ & $120(12)$ \\
\hline$C(5 A)$ & $6734(14)$ & $5840(20)$ & $6240(30)$ & $95(12)$ \\
\hline$C(6 A)$ & $5977(13)$ & $5933(13)$ & $5808(19)$ & $69(6)$ \\
\hline$C(7 A)$ & $5970(15)$ & $5002(8)$ & $3359(19)$ & $57(5)$ \\
\hline $\mathrm{C}(8 \mathrm{~A})$ & $6399(16)$ & $5284(11)$ & 2391(19) & $56(6)$ \\
\hline $\mathrm{C}(9 \mathrm{~A})$ & $6210(20)$ & $4404(10)$ & $3410(30)$ & $93(9)$ \\
\hline$C(10 A)$ & $5452(14)$ & $6365(10)$ & $6331(17)$ & $69(6)$ \\
\hline$C(11 A)$ & $5852(15)$ & $6929(12)$ & $6320(20)$ & $80(7)$ \\
\hline $\mathrm{C}(12 \mathrm{~A})$ & $5249(19)$ & $6173(13)$ & $7503(17)$ & $88(8)$ \\
\hline $\mathrm{C}(1 \mathrm{~B})$ & $5482(15)$ & $5811(9)$ & $5052(17)$ & $56(5)$ \\
\hline $\mathrm{C}(2 \mathrm{~B})$ & $6052(14)$ & $5407(10)$ & $4870(20)$ & $73(6)$ \\
\hline $\mathrm{C}(3 \mathrm{~B})$ & $6810(14)$ & $5358(9)$ & $5340(20)$ & $81(7)$ \\
\hline$C(4 B)$ & $6959(16)$ & $5716(9)$ & $6200(20)$ & $75(7)$ \\
\hline $\mathrm{C}(5 \mathrm{~B})$ & 6411(11) & $6130(8)$ & $6463(15)$ & $65(5)$ \\
\hline$C(6 B)$ & $5680(12)$ & $6167(9)$ & $5923(15)$ & $59(5)$ \\
\hline$C(7 B)$ & $5913(16)$ & $5003(9)$ & $3950(20)$ & $97(8)$ \\
\hline $\mathrm{C}(8 \mathrm{~B})$ & $6290(30)$ & $5174(14)$ & $2850(30)$ & $132(13)$ \\
\hline$C(9 B)$ & $6110(20)$ & $4402(10)$ & $4180(30)$ & $128(11)$ \\
\hline $\mathrm{C}(10 \mathrm{~B})$ & $5062(12)$ & $6603(8)$ & 6194(13) & $60(4)$ \\
\hline
\end{tabular}




\begin{tabular}{|c|c|c|c|c|}
\hline$C(11 B)$ & $5446(15)$ & $7183(9)$ & $6280(20)$ & $73(6)$ \\
\hline$C(12 B)$ & $4631(18)$ & $6482(12)$ & $7278(15)$ & $95(8)$ \\
\hline$C(1 C)$ & $3660(20)$ & $6510(8)$ & $1759(16)$ & $34(3)$ \\
\hline$C(2 C)$ & $3463(14)$ & $7066(7)$ & $1789(16)$ & $47(4)$ \\
\hline$C(3 C)$ & $3350(13)$ & $7351(8)$ & $815(16)$ & $53(5)$ \\
\hline$C(4 C)$ & $3444(13)$ & 7091(7) & $-204(17)$ & $51(5)$ \\
\hline$C(5 C)$ & $3576(13)$ & $6522(8)$ & $-223(15)$ & $44(4)$ \\
\hline$C(6 C)$ & $3700(16)$ & $6226(7)$ & $751(15)$ & $36(4)$ \\
\hline$C(7 C)$ & $3336(10)$ & $7367(7)$ & $2376(17)$ & $41(4)$ \\
\hline $\mathrm{C}(8 \mathrm{C})$ & $3626(19)$ & $7968(8)$ & $2240(20)$ & $72(8)$ \\
\hline$C(9 C)$ & $2438(11)$ & $7385(8)$ & $2670(20)$ & $56(6)$ \\
\hline$C(10 C)$ & $4001(12)$ & $5637(8)$ & 612(19) & $40(5)$ \\
\hline$C(11 C)$ & $4927(12)$ & $5541(14)$ & $700(20)$ & $51(6)$ \\
\hline $\mathrm{C}(12 \mathrm{C})$ & $3702(15)$ & $5187(12)$ & $-200(30)$ & $60(8)$ \\
\hline$C(1 D)$ & $3650(20)$ & $6458(10)$ & $1490(20)$ & $34(3)$ \\
\hline$C(2 D)$ & $3459(15)$ & 7011(9) & $1376(15)$ & $36(5)$ \\
\hline$C(3 D)$ & $3426(14)$ & $7219(10)$ & $311(18)$ & $45(6)$ \\
\hline$C(4 D)$ & $3531(17)$ & $6885(10)$ & $-600(20)$ & $62(6)$ \\
\hline$C(5 D)$ & $3672(18)$ & $6319(10)$ & $-500(19)$ & $51(6)$ \\
\hline$C(6 D)$ & $3740(30)$ & $6124(12)$ & $560(20)$ & $54(7)$ \\
\hline$C(7 D)$ & $3337(11)$ & $7334(6)$ & $2896(16)$ & $55(4)$ \\
\hline $\mathrm{C}(8 \mathrm{D})$ & $3393(16)$ & $7962(7)$ & $2840(20)$ & $69(6)$ \\
\hline$C(9 D)$ & $2536(11)$ & $7185(8)$ & $3451(19)$ & $67(5)$ \\
\hline$C(10 D)$ & $4003(18)$ & $5541(11)$ & $730(30)$ & $54(7)$ \\
\hline$C(11 D)$ & $4879(19)$ & $5630(20)$ & $360(30)$ & $59(8)$ \\
\hline$C(12 D)$ & $3450(20)$ & $5328(12)$ & $-200(30)$ & $54(8)$ \\
\hline $\mathrm{K}(1)$ & 6893(1) & $7755(1)$ & 1957(1) & $42(1)$ \\
\hline $\mathrm{O}(10)$ & $7033(7)$ & $8761(4)$ & $2847(10)$ & $135(5)$ \\
\hline $\mathrm{O}(20)$ & $6032(6)$ & $8600(5)$ & $1058(8)$ & 106(3) \\
\hline $\mathrm{O}(30)$ & $6041(7)$ & $7580(5)$ & $38(8)$ & $119(3)$ \\
\hline $\mathrm{O}(40)$ & $6940(7)$ & $6726(5)$ & $851(10)$ & $130(4)$ \\
\hline $\mathrm{O}(50)$ & 7989(8) & $6930(4)$ & $2549(8)$ & $120(4)$ \\
\hline $\mathrm{O}(60)$ & $7950(5)$ & $7928(3)$ & $3667(6)$ & $79(2)$ \\
\hline$C(10)$ & $6527(7)$ & $9150(5)$ & $2540(12)$ & $88(3)$ \\
\hline$C(20)$ & $6147(16)$ & $9069(7)$ & $1503(18)$ & 167(9) \\
\hline$C(30)$ & $5720(11)$ & $8516(8)$ & $85(12)$ & 131(6) \\
\hline
\end{tabular}




$\begin{array}{lllrl}\mathrm{C}(40) & 5603(17) & 7978(8) & -340(20) & 195(12) \\ \mathrm{C}(50) & 5903(13) & 7066(7) & -251(17) & 155(8) \\ \mathrm{C}(60) & 6464(11) & 6652(7) & 23(14) & 126(5) \\ \mathrm{C}(70) & 7542(10) & 6399(6) & 1100(12) & 108(4) \\ \mathrm{C}(80) & 7896(12) & 6451(7) & 2144(15) & 134(6) \\ \mathrm{C}(90) & 8335(12) & 6989(6) & 3531(13) & 136(7) \\ \mathrm{C}(100) & 8375(14) & 7507(6) & 3906(14) & 144(7) \\ \mathrm{C}(110) & 7963(17) & 8423(7) & 4140(20) & 235(17) \\ \mathrm{C}(120) & 7420(7) & 8840(5) & 3783(10) & 84(3) \\ \end{array}$


Table S-15. Bond lengths $[\AA]$ and angles $\left[{ }^{\circ}\right]$ for $\mathbf{4}$.

\begin{tabular}{|c|c|}
\hline $\mathrm{Fe}(1)-\mathrm{N}(11)$ & $1.912(6)$ \\
\hline $\mathrm{Fe}(1)-\mathrm{N}(21)$ & $1.932(6)$ \\
\hline $\mathrm{Fe}(1)-\mathrm{C}(1)$ & $2.024(8)$ \\
\hline $\mathrm{C}(1)-\mathrm{K}(1)$ & $3.146(8)$ \\
\hline $\mathrm{C}(1)-\mathrm{H}(1 \mathrm{~A})$ & 0.9800 \\
\hline $\mathrm{C}(1)-\mathrm{H}(1 \mathrm{~B})$ & 0.9800 \\
\hline $\mathrm{C}(1)-\mathrm{H}(1 \mathrm{C})$ & 0.9800 \\
\hline $\mathrm{N}(11)-\mathrm{C}(1 \mathrm{~B})$ & $1.31(2)$ \\
\hline $\mathrm{N}(11)-\mathrm{C}(21)$ & $1.385(11)$ \\
\hline $\mathrm{N}(11)-\mathrm{C}(1 \mathrm{~A})$ & $1.53(2)$ \\
\hline $\mathrm{N}(21)-\mathrm{C}(1 \mathrm{C})$ & $1.34(2)$ \\
\hline $\mathrm{N}(21)-\mathrm{C}(41)$ & $1.352(10)$ \\
\hline $\mathrm{N}(21)-\mathrm{C}(1 \mathrm{D})$ & $1.58(3)$ \\
\hline$C(11)-C(21)$ & $1.529(12)$ \\
\hline $\mathrm{C}(11)-\mathrm{H}(11 \mathrm{~A})$ & 0.9800 \\
\hline $\mathrm{C}(11)-\mathrm{H}(11 \mathrm{~B})$ & 0.9800 \\
\hline $\mathrm{C}(11)-\mathrm{H}(11 \mathrm{C})$ & 0.9800 \\
\hline$C(21)-C(31)$ & $1.374(12)$ \\
\hline$C(31)-C(41)$ & $1.429(12)$ \\
\hline $\mathrm{C}(31)-\mathrm{H}(31)$ & 0.9500 \\
\hline$C(41)-C(51)$ & $1.539(11)$ \\
\hline $\mathrm{C}(51)-\mathrm{H}(51 \mathrm{~A})$ & 0.9800 \\
\hline $\mathrm{C}(51)-\mathrm{H}(51 \mathrm{~B})$ & 0.9800 \\
\hline $\mathrm{C}(51)-\mathrm{H}(51 \mathrm{C})$ & 0.9800 \\
\hline $\mathrm{C}(1 \mathrm{~A})-\mathrm{C}(2 \mathrm{~A})$ & $1.409(18)$ \\
\hline $\mathrm{C}(1 \mathrm{~A})-\mathrm{C}(6 \mathrm{~A})$ & $1.414(17)$ \\
\hline $\mathrm{C}(2 \mathrm{~A})-\mathrm{C}(3 \mathrm{~A})$ & $1.393(18)$ \\
\hline $\mathrm{C}(2 \mathrm{~A})-\mathrm{C}(7 \mathrm{~A})$ & $1.51(2)$ \\
\hline $\mathrm{C}(3 \mathrm{~A})-\mathrm{C}(4 \mathrm{~A})$ & $1.407(19)$ \\
\hline $\mathrm{C}(3 \mathrm{~A})-\mathrm{H}(3 \mathrm{~A})$ & 0.9500 \\
\hline $\mathrm{C}(4 \mathrm{~A})-\mathrm{C}(5 \mathrm{~A})$ & $1.394(19)$ \\
\hline $\mathrm{C}(4 \mathrm{~A})-\mathrm{H}(4 \mathrm{~A})$ & 0.9500 \\
\hline$C(5 A)-C(6 A)$ & $1.391(19)$ \\
\hline $\mathrm{C}(5 \mathrm{~A})-\mathrm{H}(5 \mathrm{~A})$ & 0.9500 \\
\hline
\end{tabular}




\begin{tabular}{|c|c|}
\hline$C(6 A)-C(10 A)$ & $1.52(2)$ \\
\hline$C(7 A)-C(9 A)$ & $1.522(18)$ \\
\hline $\mathrm{C}(7 \mathrm{~A})-\mathrm{C}(8 \mathrm{~A})$ & $1.548(19)$ \\
\hline $\mathrm{C}(7 \mathrm{~A})-\mathrm{H}(7 \mathrm{~A})$ & 1.0000 \\
\hline $\mathrm{C}(8 \mathrm{~A})-\mathrm{H}(8 \mathrm{AA})$ & 0.9800 \\
\hline $\mathrm{C}(8 \mathrm{~A})-\mathrm{H}(8 \mathrm{AB})$ & 0.9800 \\
\hline $\mathrm{C}(8 \mathrm{~A})-\mathrm{H}(8 \mathrm{AC})$ & 0.9800 \\
\hline $\mathrm{C}(9 \mathrm{~A})-\mathrm{H}(9 \mathrm{AA})$ & 0.9800 \\
\hline $\mathrm{C}(9 \mathrm{~A})-\mathrm{H}(9 \mathrm{AB})$ & 0.9800 \\
\hline $\mathrm{C}(9 \mathrm{~A})-\mathrm{H}(9 \mathrm{AC})$ & 0.9800 \\
\hline$C(10 A)-C(11 A)$ & $1.537(19)$ \\
\hline$C(10 A)-C(12 A)$ & $1.548(19)$ \\
\hline $\mathrm{C}(10 \mathrm{~A})-\mathrm{H}(10 \mathrm{~A})$ & 1.0000 \\
\hline $\mathrm{C}(11 \mathrm{~A})-\mathrm{H}(11 \mathrm{D})$ & 0.9800 \\
\hline $\mathrm{C}(11 \mathrm{~A})-\mathrm{H}(11 \mathrm{E})$ & 0.9800 \\
\hline $\mathrm{C}(11 \mathrm{~A})-\mathrm{H}(11 \mathrm{~F})$ & 0.9800 \\
\hline $\mathrm{C}(12 \mathrm{~A})-\mathrm{H}(12 \mathrm{~A})$ & 0.9800 \\
\hline $\mathrm{C}(12 \mathrm{~A})-\mathrm{H}(12 \mathrm{~B})$ & 0.9800 \\
\hline $\mathrm{C}(12 \mathrm{~A})-\mathrm{H}(12 \mathrm{C})$ & 0.9800 \\
\hline$C(1 B)-C(2 B)$ & $1.392(18)$ \\
\hline$C(1 B)-C(6 B)$ & $1.419(18)$ \\
\hline $\mathrm{C}(2 \mathrm{~B})-\mathrm{C}(3 \mathrm{~B})$ & $1.396(17)$ \\
\hline$C(2 B)-C(7 B)$ & $1.52(2)$ \\
\hline $\mathrm{C}(3 \mathrm{~B})-\mathrm{C}(4 \mathrm{~B})$ & $1.393(18)$ \\
\hline $\mathrm{C}(3 \mathrm{~B})-\mathrm{H}(3 \mathrm{~B})$ & 0.9500 \\
\hline $\mathrm{C}(4 \mathrm{~B})-\mathrm{C}(5 \mathrm{~B})$ & $1.404(18)$ \\
\hline $\mathrm{C}(4 \mathrm{~B})-\mathrm{H}(4 \mathrm{~B})$ & 0.9500 \\
\hline $\mathrm{C}(5 \mathrm{~B})-\mathrm{C}(6 \mathrm{~B})$ & $1.390(17)$ \\
\hline $\mathrm{C}(5 \mathrm{~B})-\mathrm{H}(5 \mathrm{~B})$ & 0.9500 \\
\hline $\mathrm{C}(6 \mathrm{~B})-\mathrm{C}(10 \mathrm{~B})$ & $1.52(2)$ \\
\hline $\mathrm{C}(7 \mathrm{~B})-\mathrm{C}(9 \mathrm{~B})$ & $1.538(18)$ \\
\hline $\mathrm{C}(7 \mathrm{~B})-\mathrm{C}(8 \mathrm{~B})$ & $1.55(2)$ \\
\hline $\mathrm{C}(7 \mathrm{~B})-\mathrm{H}(7 \mathrm{~B})$ & 1.0000 \\
\hline $\mathrm{C}(8 \mathrm{~B})-\mathrm{H}(8 \mathrm{BA})$ & 0.9800 \\
\hline $\mathrm{C}(8 \mathrm{~B})-\mathrm{H}(8 \mathrm{BB})$ & 0.9800 \\
\hline $\mathrm{C}(8 \mathrm{~B})-\mathrm{H}(8 \mathrm{BC})$ & 0.9800 \\
\hline
\end{tabular}




\begin{tabular}{|c|c|}
\hline C(9B)-H(9BA) & 0.9800 \\
\hline $\mathrm{C}(9 \mathrm{~B})-\mathrm{H}(9 \mathrm{BB})$ & 0.9800 \\
\hline $\mathrm{C}(9 \mathrm{~B})-\mathrm{H}(9 \mathrm{BC})$ & 0.9800 \\
\hline $\mathrm{C}(10 \mathrm{~B})-\mathrm{C}(12 \mathrm{~B})$ & $1.538(17)$ \\
\hline$C(10 B)-C(11 B)$ & $1.568(17)$ \\
\hline $\mathrm{C}(10 \mathrm{~B})-\mathrm{H}(10 \mathrm{~B})$ & 1.0000 \\
\hline $\mathrm{C}(11 \mathrm{~B})-\mathrm{H}(11 \mathrm{G})$ & 0.9800 \\
\hline $\mathrm{C}(11 \mathrm{~B})-\mathrm{H}(11 \mathrm{H})$ & 0.9800 \\
\hline $\mathrm{C}(11 \mathrm{~B})-\mathrm{H}(11 \mathrm{I})$ & 0.9800 \\
\hline $\mathrm{C}(12 \mathrm{~B})-\mathrm{H}(12 \mathrm{D})$ & 0.9800 \\
\hline $\mathrm{C}(12 \mathrm{~B})-\mathrm{H}(12 \mathrm{E})$ & 0.9800 \\
\hline $\mathrm{C}(12 \mathrm{~B})-\mathrm{H}(12 \mathrm{~F})$ & 0.9800 \\
\hline$C(1 C)-C(2 C)$ & $1.405(16)$ \\
\hline$C(1 C)-C(6 C)$ & $1.419(16)$ \\
\hline$C(2 C)-C(3 C)$ & $1.395(16)$ \\
\hline $\mathrm{C}(2 \mathrm{C})-\mathrm{C}(7 \mathrm{D})$ & $1.520(18)$ \\
\hline$C(3 C)-C(4 C)$ & $1.410(17)$ \\
\hline $\mathrm{C}(3 \mathrm{C})-\mathrm{H}(3 \mathrm{C})$ & 0.9500 \\
\hline$C(4 C)-C(5 C)$ & $1.416(17)$ \\
\hline $\mathrm{C}(4 \mathrm{C})-\mathrm{K}(1) \# 1$ & $3.383(17)$ \\
\hline $\mathrm{C}(4 \mathrm{C})-\mathrm{H}(4 \mathrm{C})$ & 0.9500 \\
\hline$C(5 C)-C(6 C)$ & $1.412(15)$ \\
\hline $\mathrm{C}(5 \mathrm{C})-\mathrm{H}(5 \mathrm{C})$ & 0.9500 \\
\hline$C(6 C)-C(10 C)$ & $1.541(17)$ \\
\hline$C(7 C)-C(2 D)$ & $1.518(19)$ \\
\hline $\mathrm{C}(7 \mathrm{C})-\mathrm{C}(9 \mathrm{C})$ & $1.542(18)$ \\
\hline $\mathrm{C}(7 \mathrm{C})-\mathrm{C}(8 \mathrm{C})$ & $1.563(18)$ \\
\hline $\mathrm{C}(7 \mathrm{C})-\mathrm{H}(7 \mathrm{C})$ & 1.0000 \\
\hline $\mathrm{C}(8 \mathrm{C})-\mathrm{H}(8 \mathrm{CA})$ & 0.9800 \\
\hline $\mathrm{C}(8 \mathrm{C})-\mathrm{H}(8 \mathrm{CB})$ & 0.9800 \\
\hline $\mathrm{C}(8 \mathrm{C})-\mathrm{H}(8 \mathrm{CC})$ & 0.9800 \\
\hline $\mathrm{C}(9 \mathrm{C})-\mathrm{H}(9 \mathrm{CA})$ & 0.9800 \\
\hline $\mathrm{C}(9 \mathrm{C})-\mathrm{H}(9 \mathrm{CB})$ & 0.9800 \\
\hline $\mathrm{C}(9 \mathrm{C})-\mathrm{H}(9 \mathrm{CC})$ & 0.9800 \\
\hline$C(10 C)-C(12 C)$ & $1.567(17)$ \\
\hline$C(10 C)-C(11 C)$ & $1.568(17)$ \\
\hline
\end{tabular}




\begin{tabular}{|c|c|}
\hline $\mathrm{C}(10 \mathrm{C})-\mathrm{H}(10 \mathrm{C})$ & 1.0000 \\
\hline $\mathrm{C}(11 \mathrm{C})-\mathrm{H}(11 \mathrm{~J})$ & 0.9800 \\
\hline $\mathrm{C}(11 \mathrm{C})-\mathrm{H}(11 \mathrm{~K})$ & 0.9800 \\
\hline $\mathrm{C}(11 \mathrm{C})-\mathrm{H}(11 \mathrm{~L})$ & 0.9800 \\
\hline $\mathrm{C}(12 \mathrm{C})-\mathrm{H}(12 \mathrm{G})$ & 0.9800 \\
\hline $\mathrm{C}(12 \mathrm{C})-\mathrm{H}(12 \mathrm{H})$ & 0.9800 \\
\hline $\mathrm{C}(12 \mathrm{C})-\mathrm{H}(12 \mathrm{I})$ & 0.9800 \\
\hline$C(1 D)-C(2 D)$ & $1.400(17)$ \\
\hline$C(1 D)-C(6 D)$ & $1.404(17)$ \\
\hline$C(2 D)-C(3 D)$ & $1.401(17)$ \\
\hline$C(3 D)-C(4 D)$ & $1.396(18)$ \\
\hline C(3D)-H(3D) & 0.9500 \\
\hline$C(4 D)-C(5 D)$ & $1.416(19)$ \\
\hline $\mathrm{C}(4 \mathrm{D})-\mathrm{K}(1) \# 1$ & $3.32(3)$ \\
\hline C(4D)-H(4D) & 0.9500 \\
\hline$C(5 D)-C(6 D)$ & $1.393(18)$ \\
\hline C(5D)-H(5D) & 0.9500 \\
\hline$C(6 D)-C(10 D)$ & $1.51(2)$ \\
\hline C(7D)-C(9D) & $1.542(18)$ \\
\hline $\mathrm{C}(7 \mathrm{D})-\mathrm{C}(8 \mathrm{D})$ & $1.548(17)$ \\
\hline C(7D)-H(7D) & 1.0000 \\
\hline C(8D)-H(8DA) & 0.9800 \\
\hline $\mathrm{C}(8 \mathrm{D})-\mathrm{H}(8 \mathrm{DB})$ & 0.9800 \\
\hline $\mathrm{C}(8 \mathrm{D})-\mathrm{H}(8 \mathrm{DC})$ & 0.9800 \\
\hline C(9D)-H(9DA) & 0.9800 \\
\hline C(9D)-H(9DB) & 0.9800 \\
\hline C(9D)-H(9DC) & 0.9800 \\
\hline $\mathrm{C}(10 \mathrm{D})-\mathrm{C}(11 \mathrm{D})$ & $1.55(2)$ \\
\hline $\mathrm{C}(10 \mathrm{D})-\mathrm{C}(12 \mathrm{D})$ & $1.557(19)$ \\
\hline C(10D)-H(10D) & 1.0000 \\
\hline $\mathrm{C}(11 \mathrm{D})-\mathrm{H}(11 \mathrm{M})$ & 0.9800 \\
\hline $\mathrm{C}(11 \mathrm{D})-\mathrm{H}(11 \mathrm{~N})$ & 0.9800 \\
\hline C(11D)-H(11O) & 0.9800 \\
\hline $\mathrm{C}(12 \mathrm{D})-\mathrm{H}(12 \mathrm{~J})$ & 0.9800 \\
\hline $\mathrm{C}(12 \mathrm{D})-\mathrm{H}(12 \mathrm{~K})$ & 0.9800 \\
\hline $\mathrm{C}(12 \mathrm{D})-\mathrm{H}(12 \mathrm{~L})$ & 0.9800 \\
\hline
\end{tabular}




\begin{tabular}{|c|c|}
\hline $\mathrm{K}(1)-\mathrm{O}(10)$ & $2.712(9)$ \\
\hline $\mathrm{K}(1)-\mathrm{O}(20)$ & $2.756(10)$ \\
\hline $\mathrm{K}(1)-\mathrm{O}(60)$ & $2.770(7)$ \\
\hline $\mathrm{K}(1)-\mathrm{O}(30)$ & $2.779(9)$ \\
\hline $\mathrm{K}(1)-\mathrm{O}(50)$ & $2.824(10)$ \\
\hline $\mathrm{K}(1)-\mathrm{O}(40)$ & $2.870(10)$ \\
\hline $\mathrm{O}(10)-\mathrm{C}(120)$ & $1.330(13)$ \\
\hline $\mathrm{O}(10)-\mathrm{C}(10)$ & $1.330(12)$ \\
\hline $\mathrm{O}(20)-\mathrm{C}(20)$ & $1.287(15)$ \\
\hline $\mathrm{O}(20)-\mathrm{C}(30)$ & $1.316(13)$ \\
\hline $\mathrm{O}(30)-\mathrm{C}(40)$ & $1.306(16)$ \\
\hline $\mathrm{O}(30)-\mathrm{C}(50)$ & $1.333(15)$ \\
\hline $\mathrm{O}(40)-\mathrm{C}(60)$ & $1.300(14)$ \\
\hline $\mathrm{O}(40)-\mathrm{C}(70)$ & $1.322(14)$ \\
\hline $\mathrm{O}(50)-\mathrm{C}(80)$ & $1.287(14)$ \\
\hline $\mathrm{O}(50)-\mathrm{C}(90)$ & $1.341(14)$ \\
\hline $\mathrm{O}(60)-\mathrm{C}(100)$ & $1.287(13)$ \\
\hline $\mathrm{O}(60)-\mathrm{C}(110)$ & $1.347(13)$ \\
\hline$C(10)-C(20)$ & $1.432(17)$ \\
\hline $\mathrm{C}(10)-\mathrm{H}(10 \mathrm{E})$ & 0.9900 \\
\hline $\mathrm{C}(10)-\mathrm{H}(10 \mathrm{~F})$ & 0.9900 \\
\hline $\mathrm{C}(20)-\mathrm{H}(20 \mathrm{~A})$ & 0.9900 \\
\hline $\mathrm{C}(20)-\mathrm{H}(20 \mathrm{~B})$ & 0.9900 \\
\hline$C(30)-C(40)$ & $1.436(18)$ \\
\hline $\mathrm{C}(30)-\mathrm{H}(30 \mathrm{~A})$ & 0.9900 \\
\hline $\mathrm{C}(30)-\mathrm{H}(30 \mathrm{~B})$ & 0.9900 \\
\hline $\mathrm{C}(40)-\mathrm{H}(40 \mathrm{~A})$ & 0.9900 \\
\hline $\mathrm{C}(40)-\mathrm{H}(40 \mathrm{~B})$ & 0.9900 \\
\hline$C(50)-C(60)$ & $1.424(18)$ \\
\hline $\mathrm{C}(50)-\mathrm{H}(50 \mathrm{~A})$ & 0.9900 \\
\hline $\mathrm{C}(50)-\mathrm{H}(50 \mathrm{~B})$ & 0.9900 \\
\hline $\mathrm{C}(60)-\mathrm{H}(60 \mathrm{~A})$ & 0.9900 \\
\hline $\mathrm{C}(60)-\mathrm{H}(60 \mathrm{~B})$ & 0.9900 \\
\hline $\mathrm{C}(70)-\mathrm{C}(80)$ & $1.415(17)$ \\
\hline $\mathrm{C}(70)-\mathrm{H}(70 \mathrm{~A})$ & 0.9900 \\
\hline $\mathrm{C}(70)-\mathrm{H}(70 \mathrm{~B})$ & 0.9900 \\
\hline
\end{tabular}




\begin{tabular}{|c|c|}
\hline $\mathrm{C}(80)-\mathrm{H}(80 \mathrm{~A})$ & 0.9900 \\
\hline $\mathrm{C}(80)-\mathrm{H}(80 \mathrm{~B})$ & 0.9900 \\
\hline$C(90)-C(100)$ & $1.355(16)$ \\
\hline $\mathrm{C}(90)-\mathrm{H}(90 \mathrm{~A})$ & 0.9900 \\
\hline $\mathrm{C}(90)-\mathrm{H}(90 \mathrm{~B})$ & 0.9900 \\
\hline $\mathrm{C}(100)-\mathrm{H}(10 \mathrm{G})$ & 0.9900 \\
\hline $\mathrm{C}(100)-\mathrm{H}(10 \mathrm{H})$ & 0.9900 \\
\hline $\mathrm{C}(110)-\mathrm{C}(120)$ & $1.437(16)$ \\
\hline $\mathrm{C}(110)-\mathrm{H}(11 \mathrm{P})$ & 0.9900 \\
\hline $\mathrm{C}(110)-\mathrm{H}(11 \mathrm{Q})$ & 0.9900 \\
\hline $\mathrm{C}(120)-\mathrm{H}(12 \mathrm{M})$ & 0.9900 \\
\hline $\mathrm{C}(120)-\mathrm{H}(12 \mathrm{~N})$ & 0.9900 \\
\hline $\mathrm{N}(11)-\mathrm{Fe}(1)-\mathrm{N}(21)$ & $98.9(3)$ \\
\hline $\mathrm{N}(11)-\mathrm{Fe}(1)-\mathrm{C}(1)$ & $132.0(3)$ \\
\hline $\mathrm{N}(21)-\mathrm{Fe}(1)-\mathrm{C}(1)$ & $128.9(3)$ \\
\hline $\mathrm{Fe}(1)-\mathrm{C}(1)-\mathrm{K}(1)$ & $158.7(4)$ \\
\hline $\mathrm{Fe}(1)-\mathrm{C}(1)-\mathrm{H}(1 \mathrm{~A})$ & 109.5 \\
\hline $\mathrm{K}(1)-\mathrm{C}(1)-\mathrm{H}(1 \mathrm{~A})$ & 58.0 \\
\hline $\mathrm{Fe}(1)-\mathrm{C}(1)-\mathrm{H}(1 \mathrm{~B})$ & 109.5 \\
\hline $\mathrm{K}(1)-\mathrm{C}(1)-\mathrm{H}(1 \mathrm{~B})$ & 64.6 \\
\hline $\mathrm{H}(1 \mathrm{~A})-\mathrm{C}(1)-\mathrm{H}(1 \mathrm{~B})$ & 109.5 \\
\hline $\mathrm{Fe}(1)-\mathrm{C}(1)-\mathrm{H}(1 \mathrm{C})$ & 109.5 \\
\hline $\mathrm{K}(1)-\mathrm{C}(1)-\mathrm{H}(1 \mathrm{C})$ & 91.6 \\
\hline $\mathrm{H}(1 \mathrm{~A})-\mathrm{C}(1)-\mathrm{H}(1 \mathrm{C})$ & 109.5 \\
\hline $\mathrm{H}(1 \mathrm{~B})-\mathrm{C}(1)-\mathrm{H}(1 \mathrm{C})$ & 109.5 \\
\hline $\mathrm{C}(1 \mathrm{~B})-\mathrm{N}(11)-\mathrm{C}(21)$ & $119.3(12)$ \\
\hline $\mathrm{C}(21)-\mathrm{N}(11)-\mathrm{C}(1 \mathrm{~A})$ & $117.5(13)$ \\
\hline $\mathrm{C}(1 \mathrm{~B})-\mathrm{N}(11)-\mathrm{Fe}(1)$ & $118.3(11)$ \\
\hline $\mathrm{C}(21)-\mathrm{N}(11)-\mathrm{Fe}(1)$ & $121.6(6)$ \\
\hline $\mathrm{C}(1 \mathrm{~A})-\mathrm{N}(11)-\mathrm{Fe}(1)$ & $119.8(12)$ \\
\hline $\mathrm{C}(1 \mathrm{C})-\mathrm{N}(21)-\mathrm{C}(41)$ & $123.7(15)$ \\
\hline $\mathrm{C}(41)-\mathrm{N}(21)-\mathrm{C}(1 \mathrm{D})$ & $118.6(15)$ \\
\hline $\mathrm{C}(1 \mathrm{C})-\mathrm{N}(21)-\mathrm{Fe}(1)$ & $113.6(14)$ \\
\hline $\mathrm{C}(41)-\mathrm{N}(21)-\mathrm{Fe}(1)$ & $122.6(5)$ \\
\hline $\mathrm{C}(1 \mathrm{D})-\mathrm{N}(21)-\mathrm{Fe}(1)$ & $117.1(15)$ \\
\hline
\end{tabular}




\begin{tabular}{|c|c|}
\hline $\mathrm{C}(21)-\mathrm{C}(11)-\mathrm{H}(11 \mathrm{~A})$ & 109.5 \\
\hline $\mathrm{C}(21)-\mathrm{C}(11)-\mathrm{H}(11 \mathrm{~B})$ & 109.5 \\
\hline $\mathrm{H}(11 \mathrm{~A})-\mathrm{C}(11)-\mathrm{H}(11 \mathrm{~B})$ & 109.5 \\
\hline $\mathrm{C}(21)-\mathrm{C}(11)-\mathrm{H}(11 \mathrm{C})$ & 109.5 \\
\hline $\mathrm{H}(11 \mathrm{~A})-\mathrm{C}(11)-\mathrm{H}(11 \mathrm{C})$ & 109.5 \\
\hline $\mathrm{H}(11 \mathrm{~B})-\mathrm{C}(11)-\mathrm{H}(11 \mathrm{C})$ & 109.5 \\
\hline $\mathrm{C}(31)-\mathrm{C}(21)-\mathrm{N}(11)$ & $124.0(8)$ \\
\hline $\mathrm{C}(31)-\mathrm{C}(21)-\mathrm{C}(11)$ & $118.1(8)$ \\
\hline $\mathrm{N}(11)-\mathrm{C}(21)-\mathrm{C}(11)$ & $117.8(8)$ \\
\hline $\mathrm{C}(21)-\mathrm{C}(31)-\mathrm{C}(41)$ & $129.3(7)$ \\
\hline $\mathrm{C}(21)-\mathrm{C}(31)-\mathrm{H}(31)$ & 115.4 \\
\hline $\mathrm{C}(41)-\mathrm{C}(31)-\mathrm{H}(31)$ & 115.4 \\
\hline $\mathrm{N}(21)-\mathrm{C}(41)-\mathrm{C}(31)$ & $122.8(7)$ \\
\hline $\mathrm{N}(21)-\mathrm{C}(41)-\mathrm{C}(51)$ & $120.2(7)$ \\
\hline $\mathrm{C}(31)-\mathrm{C}(41)-\mathrm{C}(51)$ & $117.0(7)$ \\
\hline $\mathrm{C}(41)-\mathrm{C}(51)-\mathrm{H}(51 \mathrm{~A})$ & 109.5 \\
\hline $\mathrm{C}(41)-\mathrm{C}(51)-\mathrm{H}(51 \mathrm{~B})$ & 109.5 \\
\hline $\mathrm{H}(51 \mathrm{~A})-\mathrm{C}(51)-\mathrm{H}(51 \mathrm{~B})$ & 109.5 \\
\hline $\mathrm{C}(41)-\mathrm{C}(51)-\mathrm{H}(51 \mathrm{C})$ & 109.5 \\
\hline $\mathrm{H}(51 \mathrm{~A})-\mathrm{C}(51)-\mathrm{H}(51 \mathrm{C})$ & 109.5 \\
\hline $\mathrm{H}(51 \mathrm{~B})-\mathrm{C}(51)-\mathrm{H}(51 \mathrm{C})$ & 109.5 \\
\hline$C(2 A)-C(1 A)-C(6 A)$ & $114(2)$ \\
\hline $\mathrm{C}(2 \mathrm{~A})-\mathrm{C}(1 \mathrm{~A})-\mathrm{N}(11)$ & $125.1(15)$ \\
\hline $\mathrm{C}(6 \mathrm{~A})-\mathrm{C}(1 \mathrm{~A})-\mathrm{N}(11)$ & $120.3(17)$ \\
\hline$C(3 A)-C(2 A)-C(1 A)$ & $124(2)$ \\
\hline $\mathrm{C}(3 \mathrm{~A})-\mathrm{C}(2 \mathrm{~A})-\mathrm{C}(7 \mathrm{~A})$ & $115(2)$ \\
\hline $\mathrm{C}(1 \mathrm{~A})-\mathrm{C}(2 \mathrm{~A})-\mathrm{C}(7 \mathrm{~A})$ & $119.4(16)$ \\
\hline$C(2 A)-C(3 A)-C(4 A)$ & $117(3)$ \\
\hline $\mathrm{C}(2 \mathrm{~A})-\mathrm{C}(3 \mathrm{~A})-\mathrm{H}(3 \mathrm{~A})$ & 121.5 \\
\hline $\mathrm{C}(4 \mathrm{~A})-\mathrm{C}(3 \mathrm{~A})-\mathrm{H}(3 \mathrm{~A})$ & 121.5 \\
\hline $\mathrm{C}(5 \mathrm{~A})-\mathrm{C}(4 \mathrm{~A})-\mathrm{C}(3 \mathrm{~A})$ & $122(3)$ \\
\hline $\mathrm{C}(5 \mathrm{~A})-\mathrm{C}(4 \mathrm{~A})-\mathrm{H}(4 \mathrm{~A})$ & 119.0 \\
\hline $\mathrm{C}(3 \mathrm{~A})-\mathrm{C}(4 \mathrm{~A})-\mathrm{H}(4 \mathrm{~A})$ & 119.0 \\
\hline$C(6 A)-C(5 A)-C(4 A)$ & $118(3)$ \\
\hline $\mathrm{C}(6 \mathrm{~A})-\mathrm{C}(5 \mathrm{~A})-\mathrm{H}(5 \mathrm{~A})$ & 121.1 \\
\hline $\mathrm{C}(4 \mathrm{~A})-\mathrm{C}(5 \mathrm{~A})-\mathrm{H}(5 \mathrm{~A})$ & 121.1 \\
\hline
\end{tabular}




\begin{tabular}{|c|c|}
\hline$C(5 A)-C(6 A)-C(1 A)$ & $124(2)$ \\
\hline$C(5 A)-C(6 A)-C(10 A)$ & $119(2)$ \\
\hline$C(1 A)-C(6 A)-C(10 A)$ & $117.3(18)$ \\
\hline$C(2 A)-C(7 A)-C(9 A)$ & $109(2)$ \\
\hline$C(2 A)-C(7 A)-C(8 A)$ & $111.3(18)$ \\
\hline $\mathrm{C}(9 \mathrm{~A})-\mathrm{C}(7 \mathrm{~A})-\mathrm{C}(8 \mathrm{~A})$ & $110(2)$ \\
\hline $\mathrm{C}(2 \mathrm{~A})-\mathrm{C}(7 \mathrm{~A})-\mathrm{H}(7 \mathrm{~A})$ & 108.9 \\
\hline $\mathrm{C}(9 \mathrm{~A})-\mathrm{C}(7 \mathrm{~A})-\mathrm{H}(7 \mathrm{~A})$ & 108.9 \\
\hline $\mathrm{C}(8 \mathrm{~A})-\mathrm{C}(7 \mathrm{~A})-\mathrm{H}(7 \mathrm{~A})$ & 108.9 \\
\hline$C(7 A)-C(8 A)-H(8 A A)$ & 109.5 \\
\hline $\mathrm{C}(7 \mathrm{~A})-\mathrm{C}(8 \mathrm{~A})-\mathrm{H}(8 \mathrm{AB})$ & 109.5 \\
\hline $\mathrm{H}(8 \mathrm{AA})-\mathrm{C}(8 \mathrm{~A})-\mathrm{H}(8 \mathrm{AB})$ & 109.5 \\
\hline $\mathrm{C}(7 \mathrm{~A})-\mathrm{C}(8 \mathrm{~A})-\mathrm{H}(8 \mathrm{AC})$ & 109.5 \\
\hline $\mathrm{H}(8 \mathrm{AA})-\mathrm{C}(8 \mathrm{~A})-\mathrm{H}(8 \mathrm{AC})$ & 109.5 \\
\hline $\mathrm{H}(8 \mathrm{AB})-\mathrm{C}(8 \mathrm{~A})-\mathrm{H}(8 \mathrm{AC})$ & 109.5 \\
\hline$C(7 A)-C(9 A)-H(9 A A)$ & 109.5 \\
\hline$C(7 A)-C(9 A)-H(9 A B)$ & 109.5 \\
\hline $\mathrm{H}(9 \mathrm{AA})-\mathrm{C}(9 \mathrm{~A})-\mathrm{H}(9 \mathrm{AB})$ & 109.5 \\
\hline $\mathrm{C}(7 \mathrm{~A})-\mathrm{C}(9 \mathrm{~A})-\mathrm{H}(9 \mathrm{AC})$ & 109.5 \\
\hline H(9AA)-C(9A)-H(9AC) & 109.5 \\
\hline $\mathrm{H}(9 \mathrm{AB})-\mathrm{C}(9 \mathrm{~A})-\mathrm{H}(9 \mathrm{AC})$ & 109.5 \\
\hline$C(6 A)-C(10 A)-C(11 A)$ & $112(2)$ \\
\hline$C(6 A)-C(10 A)-C(12 A)$ & $108(2)$ \\
\hline$C(11 A)-C(10 A)-C(12 A)$ & $112.2(18)$ \\
\hline$C(6 A)-C(10 A)-H(10 A)$ & 108.2 \\
\hline $\mathrm{C}(11 \mathrm{~A})-\mathrm{C}(10 \mathrm{~A})-\mathrm{H}(10 \mathrm{~A})$ & 108.2 \\
\hline $\mathrm{C}(12 \mathrm{~A})-\mathrm{C}(10 \mathrm{~A})-\mathrm{H}(10 \mathrm{~A})$ & 108.2 \\
\hline $\mathrm{C}(10 \mathrm{~A})-\mathrm{C}(11 \mathrm{~A})-\mathrm{H}(11 \mathrm{D})$ & 109.5 \\
\hline$C(10 A)-C(11 A)-H(11 E)$ & 109.5 \\
\hline$H(11 D)-C(11 A)-H(11 E)$ & 109.5 \\
\hline $\mathrm{C}(10 \mathrm{~A})-\mathrm{C}(11 \mathrm{~A})-\mathrm{H}(11 \mathrm{~F})$ & 109.5 \\
\hline$H(11 D)-C(11 A)-H(11 F)$ & 109.5 \\
\hline $\mathrm{H}(11 \mathrm{E})-\mathrm{C}(11 \mathrm{~A})-\mathrm{H}(11 \mathrm{~F})$ & 109.5 \\
\hline $\mathrm{C}(10 \mathrm{~A})-\mathrm{C}(12 \mathrm{~A})-\mathrm{H}(12 \mathrm{~A})$ & 109.5 \\
\hline $\mathrm{C}(10 \mathrm{~A})-\mathrm{C}(12 \mathrm{~A})-\mathrm{H}(12 \mathrm{~B})$ & 109.5 \\
\hline $\mathrm{H}(12 \mathrm{~A})-\mathrm{C}(12 \mathrm{~A})-\mathrm{H}(12 \mathrm{~B})$ & 109.5 \\
\hline
\end{tabular}




\begin{tabular}{|c|c|}
\hline $\mathrm{C}(10 \mathrm{~A})-\mathrm{C}(12 \mathrm{~A})-\mathrm{H}(12 \mathrm{C})$ & 109.5 \\
\hline $\mathrm{H}(12 \mathrm{~A})-\mathrm{C}(12 \mathrm{~A})-\mathrm{H}(12 \mathrm{C})$ & 109.5 \\
\hline $\mathrm{H}(12 \mathrm{~B})-\mathrm{C}(12 \mathrm{~A})-\mathrm{H}(12 \mathrm{C})$ & 109.5 \\
\hline $\mathrm{N}(11)-\mathrm{C}(1 \mathrm{~B})-\mathrm{C}(2 \mathrm{~B})$ & $117.9(16)$ \\
\hline $\mathrm{N}(11)-\mathrm{C}(1 \mathrm{~B})-\mathrm{C}(6 \mathrm{~B})$ & $128.4(18)$ \\
\hline$C(2 B)-C(1 B)-C(6 B)$ & $113.6(19)$ \\
\hline$C(1 B)-C(2 B)-C(3 B)$ & $128(2)$ \\
\hline$C(1 B)-C(2 B)-C(7 B)$ & $118.7(16)$ \\
\hline$C(3 B)-C(2 B)-C(7 B)$ & $112.8(19)$ \\
\hline$C(4 B)-C(3 B)-C(2 B)$ & $115(2)$ \\
\hline $\mathrm{C}(4 \mathrm{~B})-\mathrm{C}(3 \mathrm{~B})-\mathrm{H}(3 \mathrm{~B})$ & 122.6 \\
\hline $\mathrm{C}(2 \mathrm{~B})-\mathrm{C}(3 \mathrm{~B})-\mathrm{H}(3 \mathrm{~B})$ & 122.6 \\
\hline$C(3 B)-C(4 B)-C(5 B)$ & $121(2)$ \\
\hline $\mathrm{C}(3 \mathrm{~B})-\mathrm{C}(4 \mathrm{~B})-\mathrm{H}(4 \mathrm{~B})$ & 119.6 \\
\hline $\mathrm{C}(5 \mathrm{~B})-\mathrm{C}(4 \mathrm{~B})-\mathrm{H}(4 \mathrm{~B})$ & 119.6 \\
\hline$C(6 B)-C(5 B)-C(4 B)$ & $120.7(19)$ \\
\hline $\mathrm{C}(6 \mathrm{~B})-\mathrm{C}(5 \mathrm{~B})-\mathrm{H}(5 \mathrm{~B})$ & 119.7 \\
\hline $\mathrm{C}(4 \mathrm{~B})-\mathrm{C}(5 \mathrm{~B})-\mathrm{H}(5 \mathrm{~B})$ & 119.7 \\
\hline$C(5 B)-C(6 B)-C(1 B)$ & $121.4(19)$ \\
\hline$C(5 B)-C(6 B)-C(10 B)$ & $122.5(17)$ \\
\hline$C(1 B)-C(6 B)-C(10 B)$ & $116.1(15)$ \\
\hline$C(2 B)-C(7 B)-C(9 B)$ & $117(2)$ \\
\hline $\mathrm{C}(2 \mathrm{~B})-\mathrm{C}(7 \mathrm{~B})-\mathrm{C}(8 \mathrm{~B})$ & $114(2)$ \\
\hline $\mathrm{C}(9 \mathrm{~B})-\mathrm{C}(7 \mathrm{~B})-\mathrm{C}(8 \mathrm{~B})$ & $109.3(18)$ \\
\hline $\mathrm{C}(2 \mathrm{~B})-\mathrm{C}(7 \mathrm{~B})-\mathrm{H}(7 \mathrm{~B})$ & 105.0 \\
\hline $\mathrm{C}(9 \mathrm{~B})-\mathrm{C}(7 \mathrm{~B})-\mathrm{H}(7 \mathrm{~B})$ & 105.0 \\
\hline $\mathrm{C}(8 \mathrm{~B})-\mathrm{C}(7 \mathrm{~B})-\mathrm{H}(7 \mathrm{~B})$ & 105.0 \\
\hline $\mathrm{C}(7 \mathrm{~B})-\mathrm{C}(8 \mathrm{~B})-\mathrm{H}(8 \mathrm{BA})$ & 109.5 \\
\hline $\mathrm{C}(7 \mathrm{~B})-\mathrm{C}(8 \mathrm{~B})-\mathrm{H}(8 \mathrm{BB})$ & 109.5 \\
\hline $\mathrm{H}(8 \mathrm{BA})-\mathrm{C}(8 \mathrm{~B})-\mathrm{H}(8 \mathrm{BB})$ & 109.5 \\
\hline $\mathrm{C}(7 \mathrm{~B})-\mathrm{C}(8 \mathrm{~B})-\mathrm{H}(8 \mathrm{BC})$ & 109.5 \\
\hline $\mathrm{H}(8 \mathrm{BA})-\mathrm{C}(8 \mathrm{~B})-\mathrm{H}(8 \mathrm{BC})$ & 109.5 \\
\hline $\mathrm{H}(8 \mathrm{BB})-\mathrm{C}(8 \mathrm{~B})-\mathrm{H}(8 \mathrm{BC})$ & 109.5 \\
\hline $\mathrm{C}(7 \mathrm{~B})-\mathrm{C}(9 \mathrm{~B})-\mathrm{H}(9 \mathrm{BA})$ & 109.5 \\
\hline $\mathrm{C}(7 \mathrm{~B})-\mathrm{C}(9 \mathrm{~B})-\mathrm{H}(9 \mathrm{BB})$ & 109.5 \\
\hline H(9BA)-C(9B)-H(9BB) & 109.5 \\
\hline
\end{tabular}




\begin{tabular}{|c|c|}
\hline $\mathrm{C}(7 \mathrm{~B})-\mathrm{C}(9 \mathrm{~B})-\mathrm{H}(9 \mathrm{BC})$ & 109.5 \\
\hline H(9BA)-C(9B)-H(9BC) & 109.5 \\
\hline H(9BB)-C(9B)-H(9BC) & 109.5 \\
\hline$C(6 B)-C(10 B)-C(12 B)$ & $111.7(19)$ \\
\hline$C(6 B)-C(10 B)-C(11 B)$ & $112.1(16)$ \\
\hline$C(12 B)-C(10 B)-C(11 B)$ & $107.8(16)$ \\
\hline $\mathrm{C}(6 \mathrm{~B})-\mathrm{C}(10 \mathrm{~B})-\mathrm{H}(10 \mathrm{~B})$ & 108.3 \\
\hline $\mathrm{C}(12 \mathrm{~B})-\mathrm{C}(10 \mathrm{~B})-\mathrm{H}(10 \mathrm{~B})$ & 108.3 \\
\hline $\mathrm{C}(11 \mathrm{~B})-\mathrm{C}(10 \mathrm{~B})-\mathrm{H}(10 \mathrm{~B})$ & 108.3 \\
\hline $\mathrm{C}(10 \mathrm{~B})-\mathrm{C}(11 \mathrm{~B})-\mathrm{H}(11 \mathrm{G})$ & 109.5 \\
\hline $\mathrm{C}(10 \mathrm{~B})-\mathrm{C}(11 \mathrm{~B})-\mathrm{H}(11 \mathrm{H})$ & 109.5 \\
\hline $\mathrm{H}(11 \mathrm{G})-\mathrm{C}(11 \mathrm{~B})-\mathrm{H}(11 \mathrm{H})$ & 109.5 \\
\hline $\mathrm{C}(10 \mathrm{~B})-\mathrm{C}(11 \mathrm{~B})-\mathrm{H}(11 \mathrm{I})$ & 109.5 \\
\hline $\mathrm{H}(11 \mathrm{G})-\mathrm{C}(11 \mathrm{~B})-\mathrm{H}(11 \mathrm{I})$ & 109.5 \\
\hline $\mathrm{H}(11 \mathrm{H})-\mathrm{C}(11 \mathrm{~B})-\mathrm{H}(11 \mathrm{I})$ & 109.5 \\
\hline $\mathrm{C}(10 \mathrm{~B})-\mathrm{C}(12 \mathrm{~B})-\mathrm{H}(12 \mathrm{D})$ & 109.5 \\
\hline $\mathrm{C}(10 \mathrm{~B})-\mathrm{C}(12 \mathrm{~B})-\mathrm{H}(12 \mathrm{E})$ & 109.5 \\
\hline $\mathrm{H}(12 \mathrm{D})-\mathrm{C}(12 \mathrm{~B})-\mathrm{H}(12 \mathrm{E})$ & 109.5 \\
\hline $\mathrm{C}(10 \mathrm{~B})-\mathrm{C}(12 \mathrm{~B})-\mathrm{H}(12 \mathrm{~F})$ & 109.5 \\
\hline $\mathrm{H}(12 \mathrm{D})-\mathrm{C}(12 \mathrm{~B})-\mathrm{H}(12 \mathrm{~F})$ & 109.5 \\
\hline $\mathrm{H}(12 \mathrm{E})-\mathrm{C}(12 \mathrm{~B})-\mathrm{H}(12 \mathrm{~F})$ & 109.5 \\
\hline $\mathrm{N}(21)-\mathrm{C}(1 \mathrm{C})-\mathrm{C}(2 \mathrm{C})$ & $121.5(14)$ \\
\hline $\mathrm{N}(21)-\mathrm{C}(1 \mathrm{C})-\mathrm{C}(6 \mathrm{C})$ & $117.6(14)$ \\
\hline$C(2 C)-C(1 C)-C(6 C)$ & $120.9(16)$ \\
\hline$C(3 C)-C(2 C)-C(1 C)$ & $119.7(16)$ \\
\hline$C(3 C)-C(2 C)-C(7 D)$ & $121.8(15)$ \\
\hline$C(1 C)-C(2 C)-C(7 D)$ & $118.4(14)$ \\
\hline$C(2 C)-C(3 C)-C(4 C)$ & $120.9(16)$ \\
\hline $\mathrm{C}(2 \mathrm{C})-\mathrm{C}(3 \mathrm{C})-\mathrm{H}(3 \mathrm{C})$ & 119.5 \\
\hline $\mathrm{C}(4 \mathrm{C})-\mathrm{C}(3 \mathrm{C})-\mathrm{H}(3 \mathrm{C})$ & 119.5 \\
\hline$C(3 C)-C(4 C)-C(5 C)$ & $118.6(16)$ \\
\hline $\mathrm{C}(3 \mathrm{C})-\mathrm{C}(4 \mathrm{C})-\mathrm{K}(1) \# 1$ & $115.1(13)$ \\
\hline $\mathrm{C}(5 \mathrm{C})-\mathrm{C}(4 \mathrm{C})-\mathrm{K}(1) \# 1$ & $102.7(11)$ \\
\hline $\mathrm{C}(3 \mathrm{C})-\mathrm{C}(4 \mathrm{C})-\mathrm{H}(4 \mathrm{C})$ & 120.7 \\
\hline $\mathrm{C}(5 \mathrm{C})-\mathrm{C}(4 \mathrm{C})-\mathrm{H}(4 \mathrm{C})$ & 120.7 \\
\hline $\mathrm{K}(1) \# 1-\mathrm{C}(4 \mathrm{C})-\mathrm{H}(4 \mathrm{C})$ & 50.9 \\
\hline
\end{tabular}




\begin{tabular}{|c|c|}
\hline$C(6 C)-C(5 C)-C(4 C)$ & $121.1(16)$ \\
\hline $\mathrm{C}(6 \mathrm{C})-\mathrm{C}(5 \mathrm{C})-\mathrm{H}(5 \mathrm{C})$ & 119.4 \\
\hline $\mathrm{C}(4 \mathrm{C})-\mathrm{C}(5 \mathrm{C})-\mathrm{H}(5 \mathrm{C})$ & 119.4 \\
\hline$C(5 C)-C(6 C)-C(1 C)$ & $118.3(15)$ \\
\hline$C(5 C)-C(6 C)-C(10 C)$ & $115.9(16)$ \\
\hline$C(1 C)-C(6 C)-C(10 C)$ & $125.1(16)$ \\
\hline$C(2 D)-C(7 C)-C(9 C)$ & $109.9(17)$ \\
\hline$C(2 D)-C(7 C)-C(8 C)$ & $114.4(17)$ \\
\hline$C(9 C)-C(7 C)-C(8 C)$ & $107.4(17)$ \\
\hline $\mathrm{C}(2 \mathrm{D})-\mathrm{C}(7 \mathrm{C})-\mathrm{H}(7 \mathrm{C})$ & 108.3 \\
\hline $\mathrm{C}(9 \mathrm{C})-\mathrm{C}(7 \mathrm{C})-\mathrm{H}(7 \mathrm{C})$ & 108.3 \\
\hline $\mathrm{C}(8 \mathrm{C})-\mathrm{C}(7 \mathrm{C})-\mathrm{H}(7 \mathrm{C})$ & 108.3 \\
\hline $\mathrm{C}(7 \mathrm{C})-\mathrm{C}(8 \mathrm{C})-\mathrm{H}(8 \mathrm{CA})$ & 109.5 \\
\hline $\mathrm{C}(7 \mathrm{C})-\mathrm{C}(8 \mathrm{C})-\mathrm{H}(8 \mathrm{CB})$ & 109.5 \\
\hline $\mathrm{H}(8 \mathrm{CA})-\mathrm{C}(8 \mathrm{C})-\mathrm{H}(8 \mathrm{CB})$ & 109.5 \\
\hline $\mathrm{C}(7 \mathrm{C})-\mathrm{C}(8 \mathrm{C})-\mathrm{H}(8 \mathrm{CC})$ & 109.5 \\
\hline $\mathrm{H}(8 \mathrm{CA})-\mathrm{C}(8 \mathrm{C})-\mathrm{H}(8 \mathrm{CC})$ & 109.5 \\
\hline $\mathrm{H}(8 \mathrm{CB})-\mathrm{C}(8 \mathrm{C})-\mathrm{H}(8 \mathrm{CC})$ & 109.5 \\
\hline $\mathrm{C}(7 \mathrm{C})-\mathrm{C}(9 \mathrm{C})-\mathrm{H}(9 \mathrm{CA})$ & 109.5 \\
\hline $\mathrm{C}(7 \mathrm{C})-\mathrm{C}(9 \mathrm{C})-\mathrm{H}(9 \mathrm{CB})$ & 109.5 \\
\hline $\mathrm{H}(9 \mathrm{CA})-\mathrm{C}(9 \mathrm{C})-\mathrm{H}(9 \mathrm{CB})$ & 109.5 \\
\hline $\mathrm{C}(7 \mathrm{C})-\mathrm{C}(9 \mathrm{C})-\mathrm{H}(9 \mathrm{CC})$ & 109.5 \\
\hline $\mathrm{H}(9 \mathrm{CA})-\mathrm{C}(9 \mathrm{C})-\mathrm{H}(9 \mathrm{CC})$ & 109.5 \\
\hline $\mathrm{H}(9 \mathrm{CB})-\mathrm{C}(9 \mathrm{C})-\mathrm{H}(9 \mathrm{CC})$ & 109.5 \\
\hline $\mathrm{C}(6 \mathrm{C})-\mathrm{C}(10 \mathrm{C})-\mathrm{C}(12 \mathrm{C})$ & $129(2)$ \\
\hline$C(6 C)-C(10 C)-C(11 C)$ & $117(2)$ \\
\hline$C(12 C)-C(10 C)-C(11 C)$ & $105(2)$ \\
\hline $\mathrm{C}(6 \mathrm{C})-\mathrm{C}(10 \mathrm{C})-\mathrm{H}(10 \mathrm{C})$ & 100.1 \\
\hline $\mathrm{C}(12 \mathrm{C})-\mathrm{C}(10 \mathrm{C})-\mathrm{H}(10 \mathrm{C})$ & 100.1 \\
\hline $\mathrm{C}(11 \mathrm{C})-\mathrm{C}(10 \mathrm{C})-\mathrm{H}(10 \mathrm{C})$ & 100.1 \\
\hline $\mathrm{C}(10 \mathrm{C})-\mathrm{C}(11 \mathrm{C})-\mathrm{H}(11 \mathrm{~J})$ & 109.5 \\
\hline $\mathrm{C}(10 \mathrm{C})-\mathrm{C}(11 \mathrm{C})-\mathrm{H}(11 \mathrm{~K})$ & 109.5 \\
\hline $\mathrm{H}(11 \mathrm{~J})-\mathrm{C}(11 \mathrm{C})-\mathrm{H}(11 \mathrm{~K})$ & 109.5 \\
\hline $\mathrm{C}(10 \mathrm{C})-\mathrm{C}(11 \mathrm{C})-\mathrm{H}(11 \mathrm{~L})$ & 109.5 \\
\hline $\mathrm{H}(11 \mathrm{~J})-\mathrm{C}(11 \mathrm{C})-\mathrm{H}(11 \mathrm{~L})$ & 109.5 \\
\hline $\mathrm{H}(11 \mathrm{~K})-\mathrm{C}(11 \mathrm{C})-\mathrm{H}(11 \mathrm{~L})$ & 109.5 \\
\hline
\end{tabular}




\begin{tabular}{|c|c|}
\hline$C(10 C)-C(12 C)-H(12 G)$ & 109.5 \\
\hline $\mathrm{C}(10 \mathrm{C})-\mathrm{C}(12 \mathrm{C})-\mathrm{H}(12 \mathrm{H})$ & 109.5 \\
\hline $\mathrm{H}(12 \mathrm{G})-\mathrm{C}(12 \mathrm{C})-\mathrm{H}(12 \mathrm{H})$ & 109.5 \\
\hline $\mathrm{C}(10 \mathrm{C})-\mathrm{C}(12 \mathrm{C})-\mathrm{H}(12 \mathrm{I})$ & 109.5 \\
\hline $\mathrm{H}(12 \mathrm{G})-\mathrm{C}(12 \mathrm{C})-\mathrm{H}(12 \mathrm{I})$ & 109.5 \\
\hline $\mathrm{H}(12 \mathrm{H})-\mathrm{C}(12 \mathrm{C})-\mathrm{H}(12 \mathrm{I})$ & 109.5 \\
\hline$C(2 D)-C(1 D)-C(6 D)$ & $121(2)$ \\
\hline $\mathrm{C}(2 \mathrm{D})-\mathrm{C}(1 \mathrm{D})-\mathrm{N}(21)$ & $118.1(16)$ \\
\hline $\mathrm{C}(6 \mathrm{D})-\mathrm{C}(1 \mathrm{D})-\mathrm{N}(21)$ & $120.7(17)$ \\
\hline $\mathrm{C}(1 \mathrm{D})-\mathrm{C}(2 \mathrm{D})-\mathrm{C}(3 \mathrm{D})$ & $116.9(19)$ \\
\hline$C(1 D)-C(2 D)-C(7 C)$ & $120.7(16)$ \\
\hline$C(3 D)-C(2 D)-C(7 C)$ & $122.4(18)$ \\
\hline$C(4 D)-C(3 D)-C(2 D)$ & $122(2)$ \\
\hline $\mathrm{C}(4 \mathrm{D})-\mathrm{C}(3 \mathrm{D})-\mathrm{H}(3 \mathrm{D})$ & 119.2 \\
\hline $\mathrm{C}(2 \mathrm{D})-\mathrm{C}(3 \mathrm{D})-\mathrm{H}(3 \mathrm{D})$ & 119.2 \\
\hline$C(3 D)-C(4 D)-C(5 D)$ & $122(2)$ \\
\hline$C(3 D)-C(4 D)-K(1) \# 1$ & $98.0(15)$ \\
\hline $\mathrm{C}(5 \mathrm{D})-\mathrm{C}(4 \mathrm{D})-\mathrm{K}(1) \# 1$ & $116.3(17)$ \\
\hline $\mathrm{C}(3 \mathrm{D})-\mathrm{C}(4 \mathrm{D})-\mathrm{H}(4 \mathrm{D})$ & 119.0 \\
\hline $\mathrm{C}(5 \mathrm{D})-\mathrm{C}(4 \mathrm{D})-\mathrm{H}(4 \mathrm{D})$ & 119.0 \\
\hline K(1)\#1-C(4D)-H(4D) & 53.1 \\
\hline $\mathrm{C}(6 \mathrm{D})-\mathrm{C}(5 \mathrm{D})-\mathrm{C}(4 \mathrm{D})$ & $116(2)$ \\
\hline $\mathrm{C}(6 \mathrm{D})-\mathrm{C}(5 \mathrm{D})-\mathrm{H}(5 \mathrm{D})$ & 122.2 \\
\hline $\mathrm{C}(4 \mathrm{D})-\mathrm{C}(5 \mathrm{D})-\mathrm{H}(5 \mathrm{D})$ & 122.2 \\
\hline$C(5 D)-C(6 D)-C(1 D)$ & $123(2)$ \\
\hline $\mathrm{C}(5 \mathrm{D})-\mathrm{C}(6 \mathrm{D})-\mathrm{C}(10 \mathrm{D})$ & $118(2)$ \\
\hline $\mathrm{C}(1 \mathrm{D})-\mathrm{C}(6 \mathrm{D})-\mathrm{C}(10 \mathrm{D})$ & $119(2)$ \\
\hline $\mathrm{C}(2 \mathrm{C})-\mathrm{C}(7 \mathrm{D})-\mathrm{C}(9 \mathrm{D})$ & $114.2(16)$ \\
\hline $\mathrm{C}(2 \mathrm{C})-\mathrm{C}(7 \mathrm{D})-\mathrm{C}(8 \mathrm{D})$ & $112.6(15)$ \\
\hline C(9D)-C(7D)-C(8D) & $107.9(16)$ \\
\hline C(2C)-C(7D)-H(7D) & 107.3 \\
\hline C(9D)-C(7D)-H(7D) & 107.3 \\
\hline $\mathrm{C}(8 \mathrm{D})-\mathrm{C}(7 \mathrm{D})-\mathrm{H}(7 \mathrm{D})$ & 107.3 \\
\hline $\mathrm{C}(7 \mathrm{D})-\mathrm{C}(8 \mathrm{D})-\mathrm{H}(8 \mathrm{DA})$ & 109.5 \\
\hline $\mathrm{C}(7 \mathrm{D})-\mathrm{C}(8 \mathrm{D})-\mathrm{H}(8 \mathrm{DB})$ & 109.5 \\
\hline $\mathrm{H}(8 \mathrm{DA})-\mathrm{C}(8 \mathrm{D})-\mathrm{H}(8 \mathrm{DB})$ & 109.5 \\
\hline
\end{tabular}




\begin{tabular}{lc} 
C(7D)-C(8D)-H(8DC) & 109.5 \\
H(8DA)-C(8D)-H(8DC) & 109.5 \\
H(8DB)-C(8D)-H(8DC) & 109.5 \\
C(7D)-C(9D)-H(9DA) & 109.5 \\
C(7D)-C(9D)-H(9DB) & 109.5 \\
H(9DA)-C(9D)-H(9DB) & 109.5 \\
C(7D)-C(9D)-H(9DC) & 109.5 \\
H(9DA)-C(9D)-H(9DC) & 109.5 \\
H(9DB)-C(9D)-H(9DC) & 109.5 \\
C(6D)-C(10D)-C(11D) & $96(3)$ \\
C(6D)-C(10D)-C(12D) & $93(2)$ \\
C(11D)-C(10D)-C(12D) & $113(3)$ \\
C(6D)-C(10D)-H(10D) & 116.9 \\
C(11D)-C(10D)-H(10D) & 116.9 \\
C(12D)-C(10D)-H(10D) & 116.9 \\
C(10D)-C(11D)-H(11M) & 109.5 \\
C(10D)-C(11D)-H(11N) & 109.5 \\
H(11M)-C(11D)-H(11N) & 109.5 \\
C(10D)-C(11D)-H(11O) & 109.5 \\
H(11M)-C(11D)-H(11O) & 109.5 \\
H(11N)-C(11D)-H(11O) & 109.5 \\
C(10D)-C(12D)-H(12J) & 109.5 \\
C(10D)-C(12D)-H(12K) & 109.5 \\
H(12J)-C(12D)-H(12K) & 109.5 \\
C(10D)-C(12D)-H(12L) & 109.5 \\
H(12J)-C(12D)-H(12L) & 109.5 \\
H(12K)-C(12D)-H(12L) & 109.5 \\
O(10)-K(1)-O(20) & $61.2(3)$ \\
O(10)-K(1)-O(60) & $60.1(2)$ \\
O(20)-K(1)-O(60) & $121.2(3)$ \\
O(10)-K(1)-O(30)-K(1)-O(50) & $121.6(3)$ \\
O(20)-K(1)-O(30) & $60.8(4)$ \\
O(60)-K(1)-O(30) & $119.2(3)$ \\
\hline
\end{tabular}




\begin{tabular}{|c|c|}
\hline $\mathrm{O}(30)-\mathrm{K}(1)-\mathrm{O}(50)$ & $116.0(3)$ \\
\hline $\mathrm{O}(10)-\mathrm{K}(1)-\mathrm{O}(40)$ & $172.2(4)$ \\
\hline $\mathrm{O}(20)-\mathrm{K}(1)-\mathrm{O}(40)$ & $119.4(4)$ \\
\hline $\mathrm{O}(60)-\mathrm{K}(1)-\mathrm{O}(40)$ & $118.3(3)$ \\
\hline $\mathrm{O}(30)-\mathrm{K}(1)-\mathrm{O}(40)$ & $58.6(4)$ \\
\hline $\mathrm{O}(50)-\mathrm{K}(1)-\mathrm{O}(40)$ & $58.1(3)$ \\
\hline $\mathrm{C}(120)-\mathrm{O}(10)-\mathrm{C}(10)$ & $116.6(11)$ \\
\hline $\mathrm{C}(120)-\mathrm{O}(10)-\mathrm{K}(1)$ & $121.4(8)$ \\
\hline $\mathrm{C}(10)-\mathrm{O}(10)-\mathrm{K}(1)$ & $119.1(8)$ \\
\hline $\mathrm{C}(20)-\mathrm{O}(20)-\mathrm{C}(30)$ & $125.6(15)$ \\
\hline $\mathrm{C}(20)-\mathrm{O}(20)-\mathrm{K}(1)$ & $115.3(10)$ \\
\hline $\mathrm{C}(30)-\mathrm{O}(20)-\mathrm{K}(1)$ & $116.7(11)$ \\
\hline $\mathrm{C}(40)-\mathrm{O}(30)-\mathrm{C}(50)$ & $121.2(14)$ \\
\hline $\mathrm{C}(40)-\mathrm{O}(30)-\mathrm{K}(1)$ & $118.2(10)$ \\
\hline $\mathrm{C}(50)-\mathrm{O}(30)-\mathrm{K}(1)$ & $117.3(10)$ \\
\hline $\mathrm{C}(60)-\mathrm{O}(40)-\mathrm{C}(70)$ & $123.9(14)$ \\
\hline $\mathrm{C}(60)-\mathrm{O}(40)-\mathrm{K}(1)$ & $118.3(11)$ \\
\hline $\mathrm{C}(70)-\mathrm{O}(40)-\mathrm{K}(1)$ & $116.6(8)$ \\
\hline $\mathrm{C}(80)-\mathrm{O}(50)-\mathrm{C}(90)$ & $119.7(13)$ \\
\hline $\mathrm{C}(80)-\mathrm{O}(50)-\mathrm{K}(1)$ & $118.6(11)$ \\
\hline $\mathrm{C}(90)-\mathrm{O}(50)-\mathrm{K}(1)$ & $115.5(8)$ \\
\hline $\mathrm{C}(100)-\mathrm{O}(60)-\mathrm{C}(110)$ & $128.2(11)$ \\
\hline $\mathrm{C}(100)-\mathrm{O}(60)-\mathrm{K}(1)$ & $113.5(9)$ \\
\hline $\mathrm{C}(110)-\mathrm{O}(60)-\mathrm{K}(1)$ & $118.3(8)$ \\
\hline $\mathrm{O}(10)-\mathrm{C}(10)-\mathrm{C}(20)$ & $115.5(15)$ \\
\hline $\mathrm{O}(10)-\mathrm{C}(10)-\mathrm{H}(10 \mathrm{E})$ & 108.4 \\
\hline $\mathrm{C}(20)-\mathrm{C}(10)-\mathrm{H}(10 \mathrm{E})$ & 108.4 \\
\hline $\mathrm{O}(10)-\mathrm{C}(10)-\mathrm{H}(10 \mathrm{~F})$ & 108.4 \\
\hline $\mathrm{C}(20)-\mathrm{C}(10)-\mathrm{H}(10 \mathrm{~F})$ & 108.4 \\
\hline $\mathrm{H}(10 \mathrm{E})-\mathrm{C}(10)-\mathrm{H}(10 \mathrm{~F})$ & 107.5 \\
\hline $\mathrm{O}(20)-\mathrm{C}(20)-\mathrm{C}(10)$ & $124.5(16)$ \\
\hline $\mathrm{O}(20)-\mathrm{C}(20)-\mathrm{K}(1)$ & $45.3(8)$ \\
\hline$C(10)-C(20)-K(1)$ & $80.3(10)$ \\
\hline $\mathrm{O}(20)-\mathrm{C}(20)-\mathrm{H}(20 \mathrm{~A})$ & 106.2 \\
\hline $\mathrm{C}(10)-\mathrm{C}(20)-\mathrm{H}(20 \mathrm{~A})$ & 106.2 \\
\hline $\mathrm{K}(1)-\mathrm{C}(20)-\mathrm{H}(20 \mathrm{~A})$ & 135.0 \\
\hline
\end{tabular}




\begin{tabular}{|c|c|}
\hline $\mathrm{O}(20)-\mathrm{C}(20)-\mathrm{H}(20 \mathrm{~B})$ & 106.2 \\
\hline$C(10)-C(20)-H(20 B)$ & 106.2 \\
\hline $\mathrm{K}(1)-\mathrm{C}(20)-\mathrm{H}(20 \mathrm{~B})$ & 114.5 \\
\hline $\mathrm{H}(20 \mathrm{~A})-\mathrm{C}(20)-\mathrm{H}(20 \mathrm{~B})$ & 106.4 \\
\hline $\mathrm{O}(20)-\mathrm{C}(30)-\mathrm{C}(40)$ & $121.8(16)$ \\
\hline $\mathrm{O}(20)-\mathrm{C}(30)-\mathrm{H}(30 \mathrm{~A})$ & 106.9 \\
\hline $\mathrm{C}(40)-\mathrm{C}(30)-\mathrm{H}(30 \mathrm{~A})$ & 106.9 \\
\hline $\mathrm{O}(20)-\mathrm{C}(30)-\mathrm{H}(30 \mathrm{~B})$ & 106.9 \\
\hline $\mathrm{C}(40)-\mathrm{C}(30)-\mathrm{H}(30 \mathrm{~B})$ & 106.9 \\
\hline $\mathrm{H}(30 \mathrm{~A})-\mathrm{C}(30)-\mathrm{H}(30 \mathrm{~B})$ & 106.7 \\
\hline $\mathrm{O}(30)-\mathrm{C}(40)-\mathrm{C}(30)$ & $118.9(16)$ \\
\hline $\mathrm{O}(30)-\mathrm{C}(40)-\mathrm{H}(40 \mathrm{~A})$ & 107.6 \\
\hline $\mathrm{C}(30)-\mathrm{C}(40)-\mathrm{H}(40 \mathrm{~A})$ & 107.6 \\
\hline $\mathrm{O}(30)-\mathrm{C}(40)-\mathrm{H}(40 \mathrm{~B})$ & 107.6 \\
\hline $\mathrm{C}(30)-\mathrm{C}(40)-\mathrm{H}(40 \mathrm{~B})$ & 107.6 \\
\hline $\mathrm{H}(40 \mathrm{~A})-\mathrm{C}(40)-\mathrm{H}(40 \mathrm{~B})$ & 107.0 \\
\hline $\mathrm{O}(30)-\mathrm{C}(50)-\mathrm{C}(60)$ & $120.1(15)$ \\
\hline $\mathrm{O}(30)-\mathrm{C}(50)-\mathrm{H}(50 \mathrm{~A})$ & 107.3 \\
\hline $\mathrm{C}(60)-\mathrm{C}(50)-\mathrm{H}(50 \mathrm{~A})$ & 107.3 \\
\hline $\mathrm{O}(30)-\mathrm{C}(50)-\mathrm{H}(50 \mathrm{~B})$ & 107.3 \\
\hline $\mathrm{C}(60)-\mathrm{C}(50)-\mathrm{H}(50 \mathrm{~B})$ & 107.3 \\
\hline $\mathrm{H}(50 \mathrm{~A})-\mathrm{C}(50)-\mathrm{H}(50 \mathrm{~B})$ & 106.9 \\
\hline $\mathrm{O}(40)-\mathrm{C}(60)-\mathrm{C}(50)$ & $119.1(15)$ \\
\hline $\mathrm{O}(40)-\mathrm{C}(60)-\mathrm{H}(60 \mathrm{~A})$ & 107.5 \\
\hline $\mathrm{C}(50)-\mathrm{C}(60)-\mathrm{H}(60 \mathrm{~A})$ & 107.5 \\
\hline $\mathrm{O}(40)-\mathrm{C}(60)-\mathrm{H}(60 \mathrm{~B})$ & 107.5 \\
\hline $\mathrm{C}(50)-\mathrm{C}(60)-\mathrm{H}(60 \mathrm{~B})$ & 107.5 \\
\hline$H(60 A)-C(60)-H(60 B)$ & 107.0 \\
\hline $\mathrm{O}(40)-\mathrm{C}(70)-\mathrm{C}(80)$ & $118.0(14)$ \\
\hline $\mathrm{O}(40)-\mathrm{C}(70)-\mathrm{H}(70 \mathrm{~A})$ & 107.8 \\
\hline $\mathrm{C}(80)-\mathrm{C}(70)-\mathrm{H}(70 \mathrm{~A})$ & 107.8 \\
\hline $\mathrm{O}(40)-\mathrm{C}(70)-\mathrm{H}(70 \mathrm{~B})$ & 107.8 \\
\hline $\mathrm{C}(80)-\mathrm{C}(70)-\mathrm{H}(70 \mathrm{~B})$ & 107.8 \\
\hline $\mathrm{H}(70 \mathrm{~A})-\mathrm{C}(70)-\mathrm{H}(70 \mathrm{~B})$ & 107.1 \\
\hline $\mathrm{O}(50)-\mathrm{C}(80)-\mathrm{C}(70)$ & $118.8(16)$ \\
\hline $\mathrm{O}(50)-\mathrm{C}(80)-\mathrm{H}(80 \mathrm{~A})$ & 107.6 \\
\hline
\end{tabular}




\begin{tabular}{|c|c|}
\hline $\mathrm{C}(70)-\mathrm{C}(80)-\mathrm{H}(80 \mathrm{~A})$ & 107.6 \\
\hline $\mathrm{O}(50)-\mathrm{C}(80)-\mathrm{H}(80 \mathrm{~B})$ & 107.6 \\
\hline $\mathrm{C}(70)-\mathrm{C}(80)-\mathrm{H}(80 \mathrm{~B})$ & 107.6 \\
\hline $\mathrm{H}(80 \mathrm{~A})-\mathrm{C}(80)-\mathrm{H}(80 \mathrm{~B})$ & 107.0 \\
\hline $\mathrm{O}(50)-\mathrm{C}(90)-\mathrm{C}(100)$ & $115.2(13)$ \\
\hline $\mathrm{O}(50)-\mathrm{C}(90)-\mathrm{H}(90 \mathrm{~A})$ & 108.5 \\
\hline $\mathrm{C}(100)-\mathrm{C}(90)-\mathrm{H}(90 \mathrm{~A})$ & 108.5 \\
\hline $\mathrm{O}(50)-\mathrm{C}(90)-\mathrm{H}(90 \mathrm{~B})$ & 108.5 \\
\hline $\mathrm{C}(100)-\mathrm{C}(90)-\mathrm{H}(90 \mathrm{~B})$ & 108.5 \\
\hline $\mathrm{H}(90 \mathrm{~A})-\mathrm{C}(90)-\mathrm{H}(90 \mathrm{~B})$ & 107.5 \\
\hline $\mathrm{O}(60)-\mathrm{C}(100)-\mathrm{C}(90)$ & $130.6(16)$ \\
\hline $\mathrm{O}(60)-\mathrm{C}(100)-\mathrm{K}(1)$ & $46.7(7)$ \\
\hline$C(90)-C(100)-K(1)$ & $84.1(9)$ \\
\hline $\mathrm{O}(60)-\mathrm{C}(100)-\mathrm{H}(10 \mathrm{G})$ & 104.6 \\
\hline $\mathrm{C}(90)-\mathrm{C}(100)-\mathrm{H}(10 \mathrm{G})$ & 104.6 \\
\hline $\mathrm{K}(1)-\mathrm{C}(100)-\mathrm{H}(10 \mathrm{G})$ & 129.8 \\
\hline $\mathrm{O}(60)-\mathrm{C}(100)-\mathrm{H}(10 \mathrm{H})$ & 104.6 \\
\hline $\mathrm{C}(90)-\mathrm{C}(100)-\mathrm{H}(10 \mathrm{H})$ & 104.6 \\
\hline $\mathrm{K}(1)-\mathrm{C}(100)-\mathrm{H}(10 \mathrm{H})$ & 120.0 \\
\hline $\mathrm{H}(10 \mathrm{G})-\mathrm{C}(100)-\mathrm{H}(10 \mathrm{H})$ & 105.7 \\
\hline $\mathrm{O}(60)-\mathrm{C}(110)-\mathrm{C}(120)$ & $120.1(12)$ \\
\hline $\mathrm{O}(60)-\mathrm{C}(110)-\mathrm{H}(11 \mathrm{P})$ & 107.3 \\
\hline $\mathrm{C}(120)-\mathrm{C}(110)-\mathrm{H}(11 \mathrm{P})$ & 107.3 \\
\hline $\mathrm{O}(60)-\mathrm{C}(110)-\mathrm{H}(11 \mathrm{Q})$ & 107.3 \\
\hline $\mathrm{C}(120)-\mathrm{C}(110)-\mathrm{H}(11 \mathrm{Q})$ & 107.3 \\
\hline $\mathrm{H}(11 \mathrm{P})-\mathrm{C}(110)-\mathrm{H}(11 \mathrm{Q})$ & 106.9 \\
\hline $\mathrm{O}(10)-\mathrm{C}(120)-\mathrm{C}(110)$ & $117.8(11)$ \\
\hline $\mathrm{O}(10)-\mathrm{C}(120)-\mathrm{H}(12 \mathrm{M})$ & 107.9 \\
\hline $\mathrm{C}(110)-\mathrm{C}(120)-\mathrm{H}(12 \mathrm{M})$ & 107.9 \\
\hline $\mathrm{O}(10)-\mathrm{C}(120)-\mathrm{H}(12 \mathrm{~N})$ & 107.9 \\
\hline $\mathrm{C}(110)-\mathrm{C}(120)-\mathrm{H}(12 \mathrm{~N})$ & 107.9 \\
\hline $\mathrm{H}(12 \mathrm{M})-\mathrm{C}(120)-\mathrm{H}(12 \mathrm{~N})$ & 107.2 \\
\hline
\end{tabular}

Symmetry transformations used to generate equivalent atoms:

\#1 x-1/2,-y+3/2,-z 
Table S-16. Anisotropic displacement parameters $\left(\AA^{2} \times 10^{3}\right)$ for 4 . The anisotropic displacement factor exponent takes the form: $-2 \pi^{2}\left[h^{2} a^{* 2} U^{11}+\ldots+2 h k a^{*} b^{*} U^{12}\right]$

\begin{tabular}{|c|c|c|c|c|c|c|}
\hline & $\mathrm{U}^{11}$ & $\mathrm{U}^{22}$ & $\mathrm{U}^{33}$ & $\mathrm{U}^{23}$ & $\mathrm{U}^{13}$ & $\mathrm{U}^{12}$ \\
\hline $\mathrm{Fe}(1)$ & $37(1)$ & $38(1)$ & $37(1)$ & $-6(1)$ & $2(1)$ & $-9(1)$ \\
\hline $\mathrm{C}(1)$ & $45(4)$ & $36(4)$ & $68(6)$ & $2(4)$ & $-2(4)$ & $-13(3)$ \\
\hline $\mathrm{N}(11)$ & $50(4)$ & $48(4)$ & $32(3)$ & $7(3)$ & $-7(3)$ & $-20(3)$ \\
\hline $\mathrm{N}(21)$ & $27(3)$ & $38(3)$ & $48(4)$ & $-9(3)$ & $-4(3)$ & $-1(3)$ \\
\hline $\mathrm{C}(11)$ & $75(7)$ & $49(5)$ & $52(5)$ & $4(4)$ & $-14(5)$ & $-29(5)$ \\
\hline$C(21)$ & $52(5)$ & $37(4)$ & $42(5)$ & $-2(4)$ & $3(4)$ & $-11(4)$ \\
\hline $\mathrm{C}(31)$ & $47(5)$ & $35(4)$ & $44(5)$ & $2(3)$ & $12(4)$ & $-6(4)$ \\
\hline $\mathrm{C}(41)$ & $29(4)$ & $38(4)$ & $45(4)$ & $-7(3)$ & $4(3)$ & $-6(3)$ \\
\hline $\mathrm{C}(51)$ & $32(4)$ & $55(5)$ & $61(5)$ & $-1(4)$ & $2(4)$ & $-4(4)$ \\
\hline $\mathrm{C}(1 \mathrm{~A})$ & $25(9)$ & $56(12)$ & $48(10)$ & $0(9)$ & $-5(7)$ & $-2(8)$ \\
\hline $\mathrm{C}(2 \mathrm{~A})$ & $47(9)$ & $74(12)$ & 41(9) & $8(8)$ & $-6(7)$ & $24(8)$ \\
\hline$C(3 A)$ & $70(11)$ & $210(30)$ & $64(14)$ & $-39(19)$ & $-23(11)$ & $62(14)$ \\
\hline $\mathrm{C}(4 \mathrm{~A})$ & $61(12)$ & $240(30)$ & $59(14)$ & $-39(18)$ & $-7(11)$ & $38(15)$ \\
\hline $\mathrm{C}(5 \mathrm{~A})$ & $39(10)$ & $190(30)$ & $53(13)$ & $-28(16)$ & $3(9)$ & $3(12)$ \\
\hline$C(6 A)$ & $42(9)$ & $114(14)$ & $50(10)$ & $-27(10)$ & $2(7)$ & $-18(9)$ \\
\hline $\mathrm{C}(7 \mathrm{~A})$ & $63(11)$ & $63(9)$ & $45(9)$ & $6(8)$ & $-4(9)$ & $15(8)$ \\
\hline $\mathrm{C}(8 \mathrm{~A})$ & $61(13)$ & $50(11)$ & $56(11)$ & $7(9)$ & $5(9)$ & $22(9)$ \\
\hline $\mathrm{C}(9 \mathrm{~A})$ & $110(20)$ & $68(10)$ & 106(19) & $21(10)$ & $39(18)$ & $33(10)$ \\
\hline$C(10 A)$ & $34(9)$ & $118(14)$ & $56(10)$ & $-46(10)$ & $-7(8)$ & $-25(9)$ \\
\hline$C(11 \mathrm{~A})$ & $35(12)$ & $123(14)$ & $81(16)$ & $-45(11)$ & $15(12)$ & $-23(10)$ \\
\hline$C(12 A)$ & $73(16)$ & $124(18)$ & $69(11)$ & $-52(11)$ & $14(10)$ & $-58(14)$ \\
\hline $\mathrm{C}(1 \mathrm{~B})$ & $71(12)$ & $60(9)$ & $36(8)$ & $24(7)$ & $-15(8)$ & $-1(7)$ \\
\hline$C(2 B)$ & $67(11)$ & $68(10)$ & $84(13)$ & $1(9)$ & $-38(9)$ & $4(8)$ \\
\hline$C(3 B)$ & $75(11)$ & $77(11)$ & $93(14)$ & $-6(10)$ & $-47(10)$ & $-3(8)$ \\
\hline $\mathrm{C}(4 \mathrm{~B})$ & $69(12)$ & $76(11)$ & $79(14)$ & $-1(10)$ & $-41(11)$ & $-11(9)$ \\
\hline$C(5 B)$ & $71(10)$ & $79(10)$ & $45(9)$ & $15(8)$ & $-30(8)$ & $-12(7)$ \\
\hline $\mathrm{C}(6 \mathrm{~B})$ & $63(10)$ & $80(9)$ & $34(8)$ & $14(6)$ & $-11(7)$ & $-13(7)$ \\
\hline$C(7 B)$ & $92(15)$ & $91(11)$ & $108(15)$ & $-23(12)$ & $-71(13)$ & $43(10)$ \\
\hline $\mathrm{C}(8 \mathrm{~B})$ & $170(30)$ & 103(19) & $122(17)$ & $-8(14)$ & $-28(16)$ & $81(18)$ \\
\hline $\mathrm{C}(9 \mathrm{~B})$ & $120(20)$ & $95(12)$ & $170(30)$ & $-13(14)$ & $-70(20)$ & $48(12)$ \\
\hline$C(10 B)$ & $59(10)$ & $86(9)$ & $37(7)$ & $-7(7)$ & $-15(7)$ & $-15(7)$ \\
\hline
\end{tabular}




\begin{tabular}{|c|c|c|c|c|c|c|}
\hline$C(11 B)$ & $66(13)$ & $95(11)$ & $58(11)$ & 2(9) & $-1(10)$ & $-14(9)$ \\
\hline $\mathrm{C}(12 \mathrm{~B})$ & $105(17)$ & $127(17)$ & $52(9)$ & $-26(10)$ & $14(10)$ & $-59(14)$ \\
\hline$C(1 C)$ & $27(4)$ & $46(4)$ & $29(8)$ & $6(4)$ & $-11(6)$ & $-5(3)$ \\
\hline$C(2 C)$ & $53(10)$ & $51(7)$ & $36(9)$ & $4(6)$ & $-19(7)$ & $1(6)$ \\
\hline$C(3 C)$ & $74(13)$ & $48(8)$ & $38(8)$ & $3(6)$ & $-27(8)$ & $-8(7)$ \\
\hline$C(4 C)$ & 71(13) & $54(8)$ & $28(9)$ & $-3(6)$ & $-27(9)$ & $-11(7)$ \\
\hline$C(5 C)$ & $46(10)$ & $52(9)$ & $33(8)$ & $9(6)$ & $-7(7)$ & $-21(7)$ \\
\hline$C(6 C)$ & $34(9)$ & $49(7)$ & $26(7)$ & $2(5)$ & $-12(6)$ & $-18(6)$ \\
\hline$C(7 C)$ & $55(10)$ & $46(8)$ & $23(9)$ & $17(7)$ & $1(7)$ & $10(6)$ \\
\hline $\mathrm{C}(8 \mathrm{C})$ & 103(18) & $59(10)$ & $55(15)$ & $0(9)$ & $19(14)$ & $-13(9)$ \\
\hline $\mathrm{C}(9 \mathrm{C})$ & $65(10)$ & $36(10)$ & $67(14)$ & $-2(9)$ & $26(9)$ & $6(7)$ \\
\hline$C(10 C)$ & $52(9)$ & $46(8)$ & $23(8)$ & $6(6)$ & $6(6)$ & $-17(6)$ \\
\hline$C(11 C)$ & $51(9)$ & $51(12)$ & $52(17)$ & $-16(12)$ & $2(7)$ & $-14(7)$ \\
\hline$C(12 C)$ & $44(12)$ & $75(15)$ & $62(14)$ & $-30(13)$ & $0(10)$ & $-8(9)$ \\
\hline$C(1 D)$ & $27(4)$ & $46(4)$ & $29(8)$ & $6(4)$ & $-11(6)$ & $-5(3)$ \\
\hline$C(2 D)$ & $39(10)$ & $48(7)$ & $20(8)$ & $12(6)$ & $-1(7)$ & $8(6)$ \\
\hline$C(3 D)$ & $56(13)$ & $56(10)$ & $23(9)$ & $15(7)$ & $0(9)$ & $11(9)$ \\
\hline$C(4 D)$ & $77(16)$ & $72(12)$ & $39(10)$ & $0(8)$ & $2(9)$ & $6(10)$ \\
\hline$C(5 D)$ & $52(13)$ & $67(11)$ & $33(9)$ & $-5(7)$ & $3(8)$ & $-1(9)$ \\
\hline$C(6 D)$ & $69(16)$ & $58(9)$ & $36(8)$ & $-3(6)$ & $-1(7)$ & $-10(8)$ \\
\hline$C(7 D)$ & $77(10)$ & $53(8)$ & $34(8)$ & $6(6)$ & $-11(7)$ & $11(6)$ \\
\hline$C(8 D)$ & $90(14)$ & $54(8)$ & $63(13)$ & $9(8)$ & $-4(11)$ & $10(7)$ \\
\hline$C(9 D)$ & $80(10)$ & $47(9)$ & $74(13)$ & 11(9) & $9(9)$ & $18(7)$ \\
\hline$C(10 D)$ & $72(14)$ & $52(10)$ & $37(12)$ & $-7(7)$ & $-9(9)$ & $-12(7)$ \\
\hline$C(11 D)$ & $79(13)$ & $61(16)$ & $37(17)$ & $-3(12)$ & $-6(9)$ & $-7(9)$ \\
\hline$C(12 D)$ & $73(18)$ & $43(12)$ & $47(13)$ & $-10(9)$ & $-16(13)$ & $-2(10)$ \\
\hline $\mathrm{K}(1)$ & $46(1)$ & $38(1)$ & $41(1)$ & $2(1)$ & $-6(1)$ & $-8(1)$ \\
\hline $\mathrm{O}(10)$ & 154(9) & $79(6)$ & $173(9)$ & $-43(6)$ & $-98(8)$ & $27(6)$ \\
\hline $\mathrm{O}(20)$ & $89(6)$ & $142(8)$ & $86(6)$ & $19(6)$ & $-22(5)$ & $18(6)$ \\
\hline $\mathrm{O}(30)$ & $127(8)$ & $163(8)$ & $65(5)$ & $-7(5)$ & $-34(5)$ & $-53(7)$ \\
\hline $\mathrm{O}(40)$ & $114(7)$ & $138(9)$ & $139(8)$ & $-84(7)$ & $-5(7)$ & $-7(6)$ \\
\hline $\mathrm{O}(50)$ & 194(12) & $77(5)$ & $90(6)$ & $4(5)$ & $-38(7)$ & $30(6)$ \\
\hline $\mathrm{O}(60)$ & $94(6)$ & $74(4)$ & $70(5)$ & $-8(4)$ & $-30(4)$ & $4(4)$ \\
\hline$C(10)$ & $58(6)$ & $77(7)$ & $128(9)$ & $30(6)$ & $3(6)$ & $4(5)$ \\
\hline $\mathrm{C}(20)$ & $210(20)$ & $110(10)$ & 179(13) & $2(9)$ & $-84(14)$ & $15(11)$ \\
\hline$C(30)$ & $121(12)$ & $178(12)$ & $93(8)$ & $24(8)$ & $-49(8)$ & $-30(10)$ \\
\hline
\end{tabular}




\begin{tabular}{lcccccc}
$\mathrm{C}(40)$ & $240(20)$ & $202(12)$ & $143(17)$ & $-19(10)$ & $-135(19)$ & $10(11)$ \\
$\mathrm{C}(50)$ & $155(15)$ & $167(11)$ & $141(15)$ & $-70(9)$ & $-68(13)$ & $-35(8)$ \\
$\mathrm{C}(60)$ & $148(11)$ & $122(10)$ & $107(10)$ & $-34(8)$ & $-4(9)$ & $-74(8)$ \\
$\mathrm{C}(70)$ & $135(10)$ & $77(8)$ & $110(9)$ & $-34(7)$ & $30(7)$ & $-10(7)$ \\
$\mathrm{C}(80)$ & $140(13)$ & $109(9)$ & $153(11)$ & $-45(8)$ & $9(9)$ & $-6(9)$ \\
$\mathrm{C}(90)$ & $191(17)$ & $89(8)$ & $127(10)$ & $-16(7)$ & $-85(12)$ & $74(9)$ \\
$\mathrm{C}(100)$ & $227(18)$ & $107(8)$ & $98(11)$ & $-23(7)$ & $-37(12)$ & $73(10)$ \\
$\mathrm{C}(110)$ & $300(30)$ & $133(10)$ & $280(20)$ & $-136(13)$ & $-240(30)$ & $112(14)$ \\
$\mathrm{C}(120)$ & $71(7)$ & $95(8)$ & $85(7)$ & $-22(6)$ & $0(5)$ & $-8(6)$ \\
& & & & & \\
\hline
\end{tabular}


Table S-17. Hydrogen coordinates ( $\times 10^{4}$ ) and isotropic displacement parameters $\left(\AA^{2} \times 10^{3}\right)$ for 4 .

\begin{tabular}{|c|c|c|c|c|}
\hline & $\mathrm{x}$ & $\mathrm{y}$ & $\mathrm{z}$ & $\mathrm{U}(\mathrm{eq})$ \\
\hline $\mathrm{H}(1 \mathrm{~A})$ & 5529 & 7144 & 2284 & 75 \\
\hline $\mathrm{H}(1 \mathrm{~B})$ & 6093 & 6959 & 3274 & 75 \\
\hline $\mathrm{H}(1 \mathrm{C})$ & 5358 & 7363 & 3493 & 75 \\
\hline $\mathrm{H}(11 \mathrm{~A})$ & 4720 & 4879 & 5512 & 88 \\
\hline $\mathrm{H}(11 \mathrm{~B})$ & 3764 & 4894 & 5605 & 88 \\
\hline $\mathrm{H}(11 \mathrm{C})$ & 4293 & 5332 & 6248 & 88 \\
\hline $\mathrm{H}(31)$ & 3105 & 5270 & 4236 & 51 \\
\hline $\mathrm{H}(51 \mathrm{~A})$ & 2147 & 6027 & 2625 & 74 \\
\hline $\mathrm{H}(51 \mathrm{~B})$ & 2271 & 5388 & 2818 & 74 \\
\hline $\mathrm{H}(51 \mathrm{C})$ & 2590 & 5666 & 1719 & 74 \\
\hline $\mathrm{H}(3 \mathrm{~A})$ & 7304 & 4918 & 4431 & 139 \\
\hline $\mathrm{H}(4 \mathrm{~A})$ & 7749 & 5399 & 5989 & 144 \\
\hline $\mathrm{H}(5 \mathrm{~A})$ & 6911 & 6023 & 6881 & 114 \\
\hline $\mathrm{H}(7 \mathrm{~A})$ & 5378 & 5029 & 3248 & 68 \\
\hline $\mathrm{H}(8 \mathrm{AA})$ & 5999 & 5425 & 1878 & 83 \\
\hline $\mathrm{H}(8 \mathrm{AB})$ & 6743 & 5020 & 2016 & 83 \\
\hline $\mathrm{H}(8 \mathrm{AC})$ & 6727 & 5585 & 2665 & 83 \\
\hline H(9AA) & 6771 & 4373 & 3614 & 140 \\
\hline $\mathrm{H}(9 \mathrm{AB})$ & 6124 & 4237 & 2689 & 140 \\
\hline $\mathrm{H}(9 \mathrm{AC})$ & 5874 & 4217 & 3951 & 140 \\
\hline $\mathrm{H}(10 \mathrm{~A})$ & 4942 & 6389 & 5906 & 83 \\
\hline $\mathrm{H}(11 \mathrm{D})$ & 6423 & 6890 & 6490 & 120 \\
\hline $\mathrm{H}(11 \mathrm{E})$ & 5597 & 7162 & 6867 & 120 \\
\hline $\mathrm{H}(11 \mathrm{~F})$ & 5791 & 7093 & 5595 & 120 \\
\hline $\mathrm{H}(12 \mathrm{~A})$ & 4893 & 5856 & 7466 & 132 \\
\hline $\mathrm{H}(12 \mathrm{~B})$ & 4980 & 6468 & 7899 & 132 \\
\hline $\mathrm{H}(12 \mathrm{C})$ & 5743 & 6074 & 7885 & 132 \\
\hline $\mathrm{H}(3 \mathrm{~B})$ & 7195 & 5101 & 5097 & 98 \\
\hline $\mathrm{H}(4 \mathrm{~B})$ & 7437 & 5679 & 6617 & 90 \\
\hline $\mathrm{H}(5 \mathrm{~B})$ & 6542 & 6387 & 7014 & 78 \\
\hline
\end{tabular}




\begin{tabular}{|c|c|c|c|c|}
\hline $\mathrm{H}(7 \mathrm{~B})$ & 5322 & 5010 & 3818 & 116 \\
\hline $\mathrm{H}(8 \mathrm{BA})$ & 5903 & 5380 & 2420 & 198 \\
\hline $\mathrm{H}(8 \mathrm{BB})$ & 6456 & 4848 & 2443 & 198 \\
\hline $\mathrm{H}(8 \mathrm{BC})$ & 6766 & 5401 & 2989 & 198 \\
\hline $\mathrm{H}(9 \mathrm{BA})$ & 6678 & 4335 & 4017 & 192 \\
\hline $\mathrm{H}(9 \mathrm{BB})$ & 5777 & 4169 & 3719 & 192 \\
\hline $\mathrm{H}(9 \mathrm{BC})$ & 6007 & 4321 & 4951 & 192 \\
\hline $\mathrm{H}(10 \mathrm{~B})$ & 4653 & 6609 & 5597 & 72 \\
\hline $\mathrm{H}(11 \mathrm{G})$ & 5039 & 7460 & 6128 & 110 \\
\hline $\mathrm{H}(11 \mathrm{H})$ & 5884 & 7216 & 5754 & 110 \\
\hline $\mathrm{H}(11 \mathrm{I})$ & 5656 & 7237 & 7023 & 110 \\
\hline $\mathrm{H}(12 \mathrm{D})$ & 5006 & 6310 & 7788 & 142 \\
\hline $\mathrm{H}(12 \mathrm{E})$ & 4179 & 6237 & 7144 & 142 \\
\hline $\mathrm{H}(12 \mathrm{~F})$ & 4433 & 6824 & 7594 & 142 \\
\hline $\mathrm{H}(3 \mathrm{C})$ & 3208 & 7725 & 838 & 64 \\
\hline $\mathrm{H}(4 \mathrm{C})$ & 3420 & 7293 & -865 & 61 \\
\hline $\mathrm{H}(5 \mathrm{C})$ & 3580 & 6335 & -903 & 52 \\
\hline $\mathrm{H}(7 \mathrm{C})$ & 3634 & 7200 & 3001 & 49 \\
\hline $\mathrm{H}(8 \mathrm{CA})$ & 3803 & 8026 & 1483 & 108 \\
\hline $\mathrm{H}(8 \mathrm{CB})$ & 4073 & 8037 & 2738 & 108 \\
\hline $\mathrm{H}(8 \mathrm{CC})$ & 3184 & 8216 & 2405 & 108 \\
\hline $\mathrm{H}(9 \mathrm{CA})$ & 2185 & 7696 & 2312 & 84 \\
\hline $\mathrm{H}(9 \mathrm{CB})$ & 2380 & 7422 & 3468 & 84 \\
\hline $\mathrm{H}(9 \mathrm{CC})$ & 2179 & 7048 & 2433 & 84 \\
\hline $\mathrm{H}(10 \mathrm{C})$ & 3821 & 5477 & 1322 & 48 \\
\hline $\mathrm{H}(11 \mathrm{~J})$ & 5195 & 5698 & 64 & 76 \\
\hline $\mathrm{H}(11 \mathrm{~K})$ & 5036 & 5149 & 731 & 76 \\
\hline $\mathrm{H}(11 \mathrm{~L})$ & 5130 & 5714 & 1368 & 76 \\
\hline $\mathrm{H}(12 \mathrm{G})$ & 3128 & 5124 & -84 & 91 \\
\hline $\mathrm{H}(12 \mathrm{H})$ & 3997 & 4848 & -62 & 91 \\
\hline $\mathrm{H}(12 \mathrm{I})$ & 3794 & 5306 & -950 & 91 \\
\hline $\mathrm{H}(3 \mathrm{D})$ & 3330 & 7597 & 206 & 54 \\
\hline $\mathrm{H}(4 \mathrm{D})$ & 3506 & 7042 & -1309 & 75 \\
\hline $\mathrm{H}(5 \mathrm{D})$ & 3716 & 6087 & -1118 & 61 \\
\hline $\mathrm{H}(7 \mathrm{D})$ & 3777 & 7205 & 3387 & 66 \\
\hline $\mathrm{H}(8 \mathrm{DA})$ & 3861 & 8067 & 2406 & 103 \\
\hline
\end{tabular}




\begin{tabular}{|c|c|c|c|c|}
\hline $\mathrm{H}(8 \mathrm{DB})$ & 3448 & 8109 & 3583 & 103 \\
\hline $\mathrm{H}(8 \mathrm{DC})$ & 2907 & 8108 & 2504 & 103 \\
\hline $\mathrm{H}(9 \mathrm{DA})$ & 2292 & 7515 & 3756 & 100 \\
\hline $\mathrm{H}(9 \mathrm{DB})$ & 2635 & 6923 & 4039 & 100 \\
\hline $\mathrm{H}(9 \mathrm{DC})$ & 2174 & 7024 & 2910 & 100 \\
\hline $\mathrm{H}(10 \mathrm{D})$ & 3920 & 5382 & 1475 & 65 \\
\hline $\mathrm{H}(11 \mathrm{M})$ & 4885 & 5814 & -348 & 88 \\
\hline $\mathrm{H}(11 \mathrm{~N})$ & 5146 & 5274 & 292 & 88 \\
\hline $\mathrm{H}(110)$ & 5162 & 5850 & 902 & 88 \\
\hline $\mathrm{H}(12 \mathrm{~J})$ & 3217 & 5638 & -593 & 81 \\
\hline $\mathrm{H}(12 \mathrm{~K})$ & 3015 & 5109 & 119 & 81 \\
\hline $\mathrm{H}(12 \mathrm{~L})$ & 3760 & 5104 & -704 & 81 \\
\hline $\mathrm{H}(10 \mathrm{E})$ & 6822 & 9499 & 2516 & 105 \\
\hline $\mathrm{H}(10 \mathrm{~F})$ & 6105 & 9184 & 3104 & 105 \\
\hline $\mathrm{H}(20 \mathrm{~A})$ & 5614 & 9243 & 1557 & 200 \\
\hline $\mathrm{H}(20 \mathrm{~B})$ & 6458 & 9285 & 969 & 200 \\
\hline $\mathrm{H}(30 \mathrm{~A})$ & 6060 & 8714 & -446 & 157 \\
\hline $\mathrm{H}(30 \mathrm{~B})$ & 5190 & 8698 & 79 & 157 \\
\hline $\mathrm{H}(40 \mathrm{~A})$ & 5034 & 7880 & -223 & 234 \\
\hline $\mathrm{H}(40 \mathrm{~B})$ & 5688 & 7995 & -1141 & 234 \\
\hline $\mathrm{H}(50 \mathrm{~A})$ & 5837 & 7060 & -1055 & 186 \\
\hline $\mathrm{H}(50 \mathrm{~B})$ & 5381 & 6960 & 68 & 186 \\
\hline $\mathrm{H}(60 \mathrm{~A})$ & 6159 & 6313 & 159 & 151 \\
\hline $\mathrm{H}(60 \mathrm{~B})$ & 6803 & 6586 & -628 & 151 \\
\hline $\mathrm{H}(70 \mathrm{~A})$ & 7967 & 6453 & 547 & 129 \\
\hline $\mathrm{H}(70 \mathrm{~B})$ & 7350 & 6019 & 1024 & 129 \\
\hline $\mathrm{H}(80 \mathrm{~A})$ & 7568 & 6238 & 2665 & 161 \\
\hline $\mathrm{H}(80 \mathrm{~B})$ & 8430 & 6277 & 2114 & 161 \\
\hline $\mathrm{H}(90 \mathrm{~A})$ & 8885 & 6841 & 3493 & 163 \\
\hline $\mathrm{H}(90 \mathrm{~B})$ & 8034 & 6768 & 4068 & 163 \\
\hline $\mathrm{H}(10 \mathrm{G})$ & 8329 & 7473 & 4710 & 173 \\
\hline $\mathrm{H}(10 \mathrm{H})$ & 8934 & 7621 & 3765 & 173 \\
\hline $\mathrm{H}(11 \mathrm{P})$ & 8513 & 8568 & 4059 & 282 \\
\hline $\mathrm{H}(11 \mathrm{Q})$ & 7872 & 8369 & 4933 & 282 \\
\hline $\mathrm{H}(12 \mathrm{M})$ & 7015 & 8893 & 4365 & 100 \\
\hline $\mathrm{H}(12 \mathrm{~N})$ & 7726 & 9184 & 3719 & 100 \\
\hline
\end{tabular}


Table S-18. Torsion angles $\left[{ }^{\circ}\right]$ for 4.

\begin{tabular}{|c|c|}
\hline $\mathrm{C}(1 \mathrm{~B})-\mathrm{N}(11)-\mathrm{C}(21)-\mathrm{C}(31)$ & $175.9(13)$ \\
\hline $\mathrm{C}(1 \mathrm{~A})-\mathrm{N}(11)-\mathrm{C}(21)-\mathrm{C}(31)$ & $-162.1(11)$ \\
\hline $\mathrm{Fe}(1)-\mathrm{N}(11)-\mathrm{C}(21)-\mathrm{C}(31)$ & $6.1(12)$ \\
\hline $\mathrm{C}(1 \mathrm{~B})-\mathrm{N}(11)-\mathrm{C}(21)-\mathrm{C}(11)$ & $0.2(16)$ \\
\hline $\mathrm{C}(1 \mathrm{~A})-\mathrm{N}(11)-\mathrm{C}(21)-\mathrm{C}(11)$ & $22.2(14)$ \\
\hline $\mathrm{Fe}(1)-\mathrm{N}(11)-\mathrm{C}(21)-\mathrm{C}(11)$ & $-169.6(6)$ \\
\hline $\mathrm{N}(11)-\mathrm{C}(21)-\mathrm{C}(31)-\mathrm{C}(41)$ & $3.0(14)$ \\
\hline $\mathrm{C}(11)-\mathrm{C}(21)-\mathrm{C}(31)-\mathrm{C}(41)$ & 178.7(8) \\
\hline $\mathrm{C}(1 \mathrm{C})-\mathrm{N}(21)-\mathrm{C}(41)-\mathrm{C}(31)$ & $175.3(12)$ \\
\hline $\mathrm{C}(1 \mathrm{D})-\mathrm{N}(21)-\mathrm{C}(41)-\mathrm{C}(31)$ & $164.7(13)$ \\
\hline $\mathrm{Fe}(1)-\mathrm{N}(21)-\mathrm{C}(41)-\mathrm{C}(31)$ & $0.1(10)$ \\
\hline $\mathrm{C}(1 \mathrm{C})-\mathrm{N}(21)-\mathrm{C}(41)-\mathrm{C}(51)$ & $-6.0(15)$ \\
\hline $\mathrm{C}(1 \mathrm{D})-\mathrm{N}(21)-\mathrm{C}(41)-\mathrm{C}(51)$ & $-16.6(15)$ \\
\hline $\mathrm{Fe}(1)-\mathrm{N}(21)-\mathrm{C}(41)-\mathrm{C}(51)$ & $178.8(6)$ \\
\hline $\mathrm{C}(21)-\mathrm{C}(31)-\mathrm{C}(41)-\mathrm{N}(21)$ & $-6.5(13)$ \\
\hline $\mathrm{C}(21)-\mathrm{C}(31)-\mathrm{C}(41)-\mathrm{C}(51)$ & $174.8(8)$ \\
\hline $\mathrm{C}(21)-\mathrm{N}(11)-\mathrm{C}(1 \mathrm{~A})-\mathrm{C}(2 \mathrm{~A})$ & $80(3)$ \\
\hline $\mathrm{Fe}(1)-\mathrm{N}(11)-\mathrm{C}(1 \mathrm{~A})-\mathrm{C}(2 \mathrm{~A})$ & $-89(3)$ \\
\hline$C(21)-N(11)-C(1 A)-C(6 A)$ & $-105(2)$ \\
\hline $\mathrm{Fe}(1)-\mathrm{N}(11)-\mathrm{C}(1 \mathrm{~A})-\mathrm{C}(6 \mathrm{~A})$ & $87(3)$ \\
\hline$C(6 A)-C(1 A)-C(2 A)-C(3 A)$ & $-5(4)$ \\
\hline $\mathrm{N}(11)-\mathrm{C}(1 \mathrm{~A})-\mathrm{C}(2 \mathrm{~A})-\mathrm{C}(3 \mathrm{~A})$ & $171(3)$ \\
\hline$C(6 A)-C(1 A)-C(2 A)-C(7 A)$ & $-172(2)$ \\
\hline $\mathrm{N}(11)-\mathrm{C}(1 \mathrm{~A})-\mathrm{C}(2 \mathrm{~A})-\mathrm{C}(7 \mathrm{~A})$ & $4(4)$ \\
\hline$C(1 A)-C(2 A)-C(3 A)-C(4 A)$ & $5(6)$ \\
\hline $\mathrm{C}(7 \mathrm{~A})-\mathrm{C}(2 \mathrm{~A})-\mathrm{C}(3 \mathrm{~A})-\mathrm{C}(4 \mathrm{~A})$ & $172(3)$ \\
\hline$C(2 A)-C(3 A)-C(4 A)-C(5 A)$ & $-1(6)$ \\
\hline$C(3 A)-C(4 A)-C(5 A)-C(6 A)$ & $-2(6)$ \\
\hline$C(4 A)-C(5 A)-C(6 A)-C(1 A)$ & $1(6)$ \\
\hline$C(4 A)-C(5 A)-C(6 A)-C(10 A)$ & $-176(3)$ \\
\hline$C(2 A)-C(1 A)-C(6 A)-C(5 A)$ & $2(5)$ \\
\hline $\mathrm{N}(11)-\mathrm{C}(1 \mathrm{~A})-\mathrm{C}(6 \mathrm{~A})-\mathrm{C}(5 \mathrm{~A})$ & $-174(3)$ \\
\hline$C(2 A)-C(1 A)-C(6 A)-C(10 A)$ & $180(2)$ \\
\hline $\mathrm{N}(11)-\mathrm{C}(1 \mathrm{~A})-\mathrm{C}(6 \mathrm{~A})-\mathrm{C}(10 \mathrm{~A})$ & $4(4)$ \\
\hline
\end{tabular}




\begin{tabular}{|c|c|}
\hline$C(3 \mathrm{~A})-\mathrm{C}(2 \mathrm{~A})-\mathrm{C}(7 \mathrm{~A})-\mathrm{C}(9 \mathrm{~A})$ & $50(4)$ \\
\hline $\mathrm{C}(1 \mathrm{~A})-\mathrm{C}(2 \mathrm{~A})-\mathrm{C}(7 \mathrm{~A})-\mathrm{C}(9 \mathrm{~A})$ & $-142(3)$ \\
\hline$C(3 A)-C(2 A)-C(7 A)-C(8 A)$ & $-72(3)$ \\
\hline $\mathrm{C}(1 \mathrm{~A})-\mathrm{C}(2 \mathrm{~A})-\mathrm{C}(7 \mathrm{~A})-\mathrm{C}(8 \mathrm{~A})$ & $97(3)$ \\
\hline$C(5 A)-C(6 A)-C(10 A)-C(11 A)$ & $59(4)$ \\
\hline$C(1 A)-C(6 A)-C(10 A)-C(11 A)$ & $-119(3)$ \\
\hline$C(5 A)-C(6 A)-C(10 A)-C(12 A)$ & $-65(4)$ \\
\hline$C(1 A)-C(6 A)-C(10 A)-C(12 A)$ & $117(3)$ \\
\hline $\mathrm{C}(21)-\mathrm{N}(11)-\mathrm{C}(1 \mathrm{~B})-\mathrm{C}(2 \mathrm{~B})$ & $83(2)$ \\
\hline $\mathrm{Fe}(1)-\mathrm{N}(11)-\mathrm{C}(1 \mathrm{~B})-\mathrm{C}(2 \mathrm{~B})$ & $-107(2)$ \\
\hline $\mathrm{C}(21)-\mathrm{N}(11)-\mathrm{C}(1 \mathrm{~B})-\mathrm{C}(6 \mathrm{~B})$ & $-100(2)$ \\
\hline $\mathrm{Fe}(1)-\mathrm{N}(11)-\mathrm{C}(1 \mathrm{~B})-\mathrm{C}(6 \mathrm{~B})$ & $70(3)$ \\
\hline $\mathrm{N}(11)-\mathrm{C}(1 \mathrm{~B})-\mathrm{C}(2 \mathrm{~B})-\mathrm{C}(3 \mathrm{~B})$ & $169(3)$ \\
\hline $\mathrm{C}(6 \mathrm{~B})-\mathrm{C}(1 \mathrm{~B})-\mathrm{C}(2 \mathrm{~B})-\mathrm{C}(3 \mathrm{~B})$ & $-8(4)$ \\
\hline $\mathrm{N}(11)-\mathrm{C}(1 \mathrm{~B})-\mathrm{C}(2 \mathrm{~B})-\mathrm{C}(7 \mathrm{~B})$ & $-2(4)$ \\
\hline$C(6 B)-C(1 B)-C(2 B)-C(7 B)$ & $-179(2)$ \\
\hline $\mathrm{C}(1 \mathrm{~B})-\mathrm{C}(2 \mathrm{~B})-\mathrm{C}(3 \mathrm{~B})-\mathrm{C}(4 \mathrm{~B})$ & $10(4)$ \\
\hline $\mathrm{C}(7 \mathrm{~B})-\mathrm{C}(2 \mathrm{~B})-\mathrm{C}(3 \mathrm{~B})-\mathrm{C}(4 \mathrm{~B})$ & $-179(2)$ \\
\hline $\mathrm{C}(2 \mathrm{~B})-\mathrm{C}(3 \mathrm{~B})-\mathrm{C}(4 \mathrm{~B})-\mathrm{C}(5 \mathrm{~B})$ & $-7(4)$ \\
\hline $\mathrm{C}(3 \mathrm{~B})-\mathrm{C}(4 \mathrm{~B})-\mathrm{C}(5 \mathrm{~B})-\mathrm{C}(6 \mathrm{~B})$ & $5(4)$ \\
\hline $\mathrm{C}(4 \mathrm{~B})-\mathrm{C}(5 \mathrm{~B})-\mathrm{C}(6 \mathrm{~B})-\mathrm{C}(1 \mathrm{~B})$ & $-4(3)$ \\
\hline $\mathrm{C}(4 \mathrm{~B})-\mathrm{C}(5 \mathrm{~B})-\mathrm{C}(6 \mathrm{~B})-\mathrm{C}(10 \mathrm{~B})$ & $179(2)$ \\
\hline $\mathrm{N}(11)-\mathrm{C}(1 \mathrm{~B})-\mathrm{C}(6 \mathrm{~B})-\mathrm{C}(5 \mathrm{~B})$ & $-172(2)$ \\
\hline $\mathrm{C}(2 \mathrm{~B})-\mathrm{C}(1 \mathrm{~B})-\mathrm{C}(6 \mathrm{~B})-\mathrm{C}(5 \mathrm{~B})$ & $5(3)$ \\
\hline $\mathrm{N}(11)-\mathrm{C}(1 \mathrm{~B})-\mathrm{C}(6 \mathrm{~B})-\mathrm{C}(10 \mathrm{~B})$ & $6(3)$ \\
\hline $\mathrm{C}(2 \mathrm{~B})-\mathrm{C}(1 \mathrm{~B})-\mathrm{C}(6 \mathrm{~B})-\mathrm{C}(10 \mathrm{~B})$ & $-177(2)$ \\
\hline $\mathrm{C}(1 \mathrm{~B})-\mathrm{C}(2 \mathrm{~B})-\mathrm{C}(7 \mathrm{~B})-\mathrm{C}(9 \mathrm{~B})$ & $-138(3)$ \\
\hline $\mathrm{C}(3 \mathrm{~B})-\mathrm{C}(2 \mathrm{~B})-\mathrm{C}(7 \mathrm{~B})-\mathrm{C}(9 \mathrm{~B})$ & $50(3)$ \\
\hline $\mathrm{C}(1 \mathrm{~B})-\mathrm{C}(2 \mathrm{~B})-\mathrm{C}(7 \mathrm{~B})-\mathrm{C}(8 \mathrm{~B})$ & $92(3)$ \\
\hline $\mathrm{C}(3 \mathrm{~B})-\mathrm{C}(2 \mathrm{~B})-\mathrm{C}(7 \mathrm{~B})-\mathrm{C}(8 \mathrm{~B})$ & $-80(3)$ \\
\hline $\mathrm{C}(5 \mathrm{~B})-\mathrm{C}(6 \mathrm{~B})-\mathrm{C}(10 \mathrm{~B})-\mathrm{C}(12 \mathrm{~B})$ & $-74(2)$ \\
\hline $\mathrm{C}(1 \mathrm{~B})-\mathrm{C}(6 \mathrm{~B})-\mathrm{C}(10 \mathrm{~B})-\mathrm{C}(12 \mathrm{~B})$ & $108(2)$ \\
\hline $\mathrm{C}(5 \mathrm{~B})-\mathrm{C}(6 \mathrm{~B})-\mathrm{C}(10 \mathrm{~B})-\mathrm{C}(11 \mathrm{~B})$ & $47(2)$ \\
\hline $\mathrm{C}(1 \mathrm{~B})-\mathrm{C}(6 \mathrm{~B})-\mathrm{C}(10 \mathrm{~B})-\mathrm{C}(11 \mathrm{~B})$ & $-130.8(19)$ \\
\hline $\mathrm{C}(41)-\mathrm{N}(21)-\mathrm{C}(1 \mathrm{C})-\mathrm{C}(2 \mathrm{C})$ & $112(2)$ \\
\hline $\mathrm{Fe}(1)-\mathrm{N}(21)-\mathrm{C}(1 \mathrm{C})-\mathrm{C}(2 \mathrm{C})$ & $-72(3)$ \\
\hline
\end{tabular}




\begin{tabular}{|c|c|}
\hline $\mathrm{C}(41)-\mathrm{N}(21)-\mathrm{C}(1 \mathrm{C})-\mathrm{C}(6 \mathrm{C})$ & $-68(3)$ \\
\hline $\mathrm{Fe}(1)-\mathrm{N}(21)-\mathrm{C}(1 \mathrm{C})-\mathrm{C}(6 \mathrm{C})$ & $108(2)$ \\
\hline $\mathrm{N}(21)-\mathrm{C}(1 \mathrm{C})-\mathrm{C}(2 \mathrm{C})-\mathrm{C}(3 \mathrm{C})$ & $177(2)$ \\
\hline$C(6 C)-C(1 C)-C(2 C)-C(3 C)$ & $-3(4)$ \\
\hline $\mathrm{N}(21)-\mathrm{C}(1 \mathrm{C})-\mathrm{C}(2 \mathrm{C})-\mathrm{C}(7 \mathrm{D})$ & $-6(4)$ \\
\hline$C(6 C)-C(1 C)-C(2 C)-C(7 D)$ & $174(2)$ \\
\hline$C(1 C)-C(2 C)-C(3 C)-C(4 C)$ & $-1(3)$ \\
\hline$C(7 D)-C(2 C)-C(3 C)-C(4 C)$ & $-178(2)$ \\
\hline $\mathrm{C}(2 \mathrm{C})-\mathrm{C}(3 \mathrm{C})-\mathrm{C}(4 \mathrm{C})-\mathrm{C}(5 \mathrm{C})$ & $6(3)$ \\
\hline$C(2 C)-C(3 C)-C(4 C)-K(1) \# 1$ & $128.5(17)$ \\
\hline$C(3 C)-C(4 C)-C(5 C)-C(6 C)$ & $-7(3)$ \\
\hline $\mathrm{K}(1) \# 1-\mathrm{C}(4 \mathrm{C})-\mathrm{C}(5 \mathrm{C})-\mathrm{C}(6 \mathrm{C})$ & $-135.2(18)$ \\
\hline$C(4 C)-C(5 C)-C(6 C)-C(1 C)$ & $3(3)$ \\
\hline$C(4 C)-C(5 C)-C(6 C)-C(10 C)$ & $-168.3(19)$ \\
\hline $\mathrm{N}(21)-\mathrm{C}(1 \mathrm{C})-\mathrm{C}(6 \mathrm{C})-\mathrm{C}(5 \mathrm{C})$ & $-178(2)$ \\
\hline$C(2 C)-C(1 C)-C(6 C)-C(5 C)$ & $2(4)$ \\
\hline$N(21)-C(1 C)-C(6 C)-C(10 C)$ & $-8(4)$ \\
\hline$C(2 C)-C(1 C)-C(6 C)-C(10 C)$ & $172(3)$ \\
\hline$C(5 C)-C(6 C)-C(10 C)-C(12 C)$ & $-45(3)$ \\
\hline$C(1 C)-C(6 C)-C(10 C)-C(12 C)$ & $145(3)$ \\
\hline$C(5 C)-C(6 C)-C(10 C)-C(11 C)$ & $96(3)$ \\
\hline$C(1 C)-C(6 C)-C(10 C)-C(11 C)$ & $-74(3)$ \\
\hline $\mathrm{C}(41)-\mathrm{N}(21)-\mathrm{C}(1 \mathrm{D})-\mathrm{C}(2 \mathrm{D})$ & $119(3)$ \\
\hline $\mathrm{Fe}(1)-\mathrm{N}(21)-\mathrm{C}(1 \mathrm{D})-\mathrm{C}(2 \mathrm{D})$ & $-75(3)$ \\
\hline $\mathrm{C}(41)-\mathrm{N}(21)-\mathrm{C}(1 \mathrm{D})-\mathrm{C}(6 \mathrm{D})$ & $-65(3)$ \\
\hline $\mathrm{Fe}(1)-\mathrm{N}(21)-\mathrm{C}(1 \mathrm{D})-\mathrm{C}(6 \mathrm{D})$ & $100(3)$ \\
\hline $\mathrm{C}(6 \mathrm{D})-\mathrm{C}(1 \mathrm{D})-\mathrm{C}(2 \mathrm{D})-\mathrm{C}(3 \mathrm{D})$ & $-4(5)$ \\
\hline $\mathrm{N}(21)-\mathrm{C}(1 \mathrm{D})-\mathrm{C}(2 \mathrm{D})-\mathrm{C}(3 \mathrm{D})$ & $172(2)$ \\
\hline $\mathrm{C}(6 \mathrm{D})-\mathrm{C}(1 \mathrm{D})-\mathrm{C}(2 \mathrm{D})-\mathrm{C}(7 \mathrm{C})$ & $178(3)$ \\
\hline $\mathrm{N}(21)-\mathrm{C}(1 \mathrm{D})-\mathrm{C}(2 \mathrm{D})-\mathrm{C}(7 \mathrm{C})$ & $-6(4)$ \\
\hline $\mathrm{C}(9 \mathrm{C})-\mathrm{C}(7 \mathrm{C})-\mathrm{C}(2 \mathrm{D})-\mathrm{C}(1 \mathrm{D})$ & $-93(3)$ \\
\hline $\mathrm{C}(8 \mathrm{C})-\mathrm{C}(7 \mathrm{C})-\mathrm{C}(2 \mathrm{D})-\mathrm{C}(1 \mathrm{D})$ & $146(3)$ \\
\hline $\mathrm{C}(9 \mathrm{C})-\mathrm{C}(7 \mathrm{C})-\mathrm{C}(2 \mathrm{D})-\mathrm{C}(3 \mathrm{D})$ & $89(3)$ \\
\hline $\mathrm{C}(8 \mathrm{C})-\mathrm{C}(7 \mathrm{C})-\mathrm{C}(2 \mathrm{D})-\mathrm{C}(3 \mathrm{D})$ & $-32(3)$ \\
\hline $\mathrm{C}(1 \mathrm{D})-\mathrm{C}(2 \mathrm{D})-\mathrm{C}(3 \mathrm{D})-\mathrm{C}(4 \mathrm{D})$ & $3(4)$ \\
\hline$C(7 C)-C(2 D)-C(3 D)-C(4 D)$ & $-179(2)$ \\
\hline
\end{tabular}




\begin{tabular}{|c|c|}
\hline$C(2 D)-C(3 D)-C(4 D)-C(5 D)$ & $0(4)$ \\
\hline$C(2 D)-C(3 D)-C(4 D)-K(1) \# 1$ & $128(2)$ \\
\hline$C(3 D)-C(4 D)-C(5 D)-C(6 D)$ & $-3(4)$ \\
\hline $\mathrm{K}(1) \# 1-\mathrm{C}(4 \mathrm{D})-\mathrm{C}(5 \mathrm{D})-\mathrm{C}(6 \mathrm{D})$ & $-123(3)$ \\
\hline$C(4 D)-C(5 D)-C(6 D)-C(1 D)$ & $3(5)$ \\
\hline$C(4 D)-C(5 D)-C(6 D)-C(10 D)$ & $-172(3)$ \\
\hline$C(2 D)-C(1 D)-C(6 D)-C(5 D)$ & $1(6)$ \\
\hline $\mathrm{N}(21)-\mathrm{C}(1 \mathrm{D})-\mathrm{C}(6 \mathrm{D})-\mathrm{C}(5 \mathrm{D})$ & $-175(3)$ \\
\hline$C(2 D)-C(1 D)-C(6 D)-C(10 D)$ & $175(4)$ \\
\hline$N(21)-C(1 D)-C(6 D)-C(10 D)$ & $0(5)$ \\
\hline$C(3 C)-C(2 C)-C(7 D)-C(9 D)$ & $102(2)$ \\
\hline$C(1 C)-C(2 C)-C(7 D)-C(9 D)$ & $-75(3)$ \\
\hline$C(3 C)-C(2 C)-C(7 D)-C(8 D)$ & $-21(3)$ \\
\hline$C(1 C)-C(2 C)-C(7 D)-C(8 D)$ & $162(2)$ \\
\hline$C(5 D)-C(6 D)-C(10 D)-C(11 D)$ & $70(4)$ \\
\hline$C(1 D)-C(6 D)-C(10 D)-C(11 D)$ & $-104(4)$ \\
\hline$C(5 D)-C(6 D)-C(10 D)-C(12 D)$ & $-43(4)$ \\
\hline$C(1 D)-C(6 D)-C(10 D)-C(12 D)$ & $142(4)$ \\
\hline $\mathrm{C}(120)-\mathrm{O}(10)-\mathrm{C}(10)-\mathrm{C}(20)$ & $177.0(17)$ \\
\hline $\mathrm{K}(1)-\mathrm{O}(10)-\mathrm{C}(10)-\mathrm{C}(20)$ & $-22(2)$ \\
\hline $\mathrm{C}(30)-\mathrm{O}(20)-\mathrm{C}(20)-\mathrm{C}(10)$ & $-177(2)$ \\
\hline $\mathrm{K}(1)-\mathrm{O}(20)-\mathrm{C}(20)-\mathrm{C}(10)$ & $-15(3)$ \\
\hline $\mathrm{C}(30)-\mathrm{O}(20)-\mathrm{C}(20)-\mathrm{K}(1)$ & $-162(2)$ \\
\hline $\mathrm{O}(10)-\mathrm{C}(10)-\mathrm{C}(20)-\mathrm{O}(20)$ & $25(3)$ \\
\hline $\mathrm{O}(10)-\mathrm{C}(10)-\mathrm{C}(20)-\mathrm{K}(1)$ & $14.8(14)$ \\
\hline $\mathrm{C}(20)-\mathrm{O}(20)-\mathrm{C}(30)-\mathrm{C}(40)$ & $-179(3)$ \\
\hline $\mathrm{K}(1)-\mathrm{O}(20)-\mathrm{C}(30)-\mathrm{C}(40)$ & $19(3)$ \\
\hline $\mathrm{C}(50)-\mathrm{O}(30)-\mathrm{C}(40)-\mathrm{C}(30)$ & $173(2)$ \\
\hline $\mathrm{K}(1)-\mathrm{O}(30)-\mathrm{C}(40)-\mathrm{C}(30)$ & $14(3)$ \\
\hline $\mathrm{O}(20)-\mathrm{C}(30)-\mathrm{C}(40)-\mathrm{O}(30)$ & $-24(4)$ \\
\hline $\mathrm{C}(40)-\mathrm{O}(30)-\mathrm{C}(50)-\mathrm{C}(60)$ & $170(2)$ \\
\hline $\mathrm{K}(1)-\mathrm{O}(30)-\mathrm{C}(50)-\mathrm{C}(60)$ & $-31(3)$ \\
\hline $\mathrm{C}(70)-\mathrm{O}(40)-\mathrm{C}(60)-\mathrm{C}(50)$ & $-172(2)$ \\
\hline $\mathrm{K}(1)-\mathrm{O}(40)-\mathrm{C}(60)-\mathrm{C}(50)$ & $-4(2)$ \\
\hline $\mathrm{O}(30)-\mathrm{C}(50)-\mathrm{C}(60)-\mathrm{O}(40)$ & $24(3)$ \\
\hline $\mathrm{C}(60)-\mathrm{O}(40)-\mathrm{C}(70)-\mathrm{C}(80)$ & $-165.9(17)$ \\
\hline
\end{tabular}


$\mathrm{K}(1)-\mathrm{O}(40)-\mathrm{C}(70)-\mathrm{C}(80)$

$\mathrm{C}(90)-\mathrm{O}(50)-\mathrm{C}(80)-\mathrm{C}(70)$

$\mathrm{K}(1)-\mathrm{O}(50)-\mathrm{C}(80)-\mathrm{C}(70)$

$\mathrm{O}(40)-\mathrm{C}(70)-\mathrm{C}(80)-\mathrm{O}(50)$

$\mathrm{C}(80)-\mathrm{O}(50)-\mathrm{C}(90)-\mathrm{C}(100)$

$\mathrm{K}(1)-\mathrm{O}(50)-\mathrm{C}(90)-\mathrm{C}(100)$

$\mathrm{C}(110)-\mathrm{O}(60)-\mathrm{C}(100)-\mathrm{C}(90)$

$\mathrm{K}(1)-\mathrm{O}(60)-\mathrm{C}(100)-\mathrm{C}(90)$

$\mathrm{C}(110)-\mathrm{O}(60)-\mathrm{C}(100)-\mathrm{K}(1)$

$\mathrm{O}(50)-\mathrm{C}(90)-\mathrm{C}(100)-\mathrm{O}(60)$

$\mathrm{O}(50)-\mathrm{C}(90)-\mathrm{C}(100)-\mathrm{K}(1)$

$\mathrm{C}(100)-\mathrm{O}(60)-\mathrm{C}(110)-\mathrm{C}(120)$

$\mathrm{K}(1)-\mathrm{O}(60)-\mathrm{C}(110)-\mathrm{C}(120)$

C(10)-O(10)-C(120)-C(110)

$\mathrm{K}(1)-\mathrm{O}(10)-\mathrm{C}(120)-\mathrm{C}(110)$

$\mathrm{O}(60)-\mathrm{C}(110)-\mathrm{C}(120)-\mathrm{O}(10)$
27(2)

$-179.0(19)$

$30(2)$

$-39(3)$

$-177(2)$

$-25(3)$

173(3)

$-7(3)$

180(3)

23(4)

18.2(18)

$-178(2)$

2(4)

179(2)

19(2)

$-14(4)$

Symmetry transformations used to generate equivalent atoms:

$\# 1 \mathrm{x}-1 / 2,-\mathrm{y}+3 / 2,-\mathrm{z}$ 
$\left[\mathrm{L}^{\mathrm{Me}} \mathrm{FeCH}_{3}\right][\mathbf{K}(\mathbf{1 8}$-crown-6)(12-crown-4)] (4') We also crystallographically characterized a crystal in which the potassium is bound by one 18-crown-6 and one 12-crown 4. Low-temperature diffraction data ( $\omega$-scans) were collected on a Rigaku MicroMax-007HF diffractometer coupled to a Saturn994+ CCD detector with $\mathrm{Cu} \mathrm{K \alpha}(\lambda=1.54178 \AA)$ for the structure of 4'. The diffraction images were processed and scaled using Rigaku Oxford Diffraction software (CrysAlisPro; Rigaku OD: The Woodlands, TX, 2015). The structure was solved with SHELXT and was refined against $\mathrm{F}^{2}$ on all data by full-matrix least squares with SHELXL (Sheldrick, G. M. Acta Cryst. 2008, A64, 112-122). All non-hydrogen atoms were refined anisotropically. Hydrogen atoms were included in the model at geometrically calculated positions and refined using a riding model. The isotropic displacement parameters of all hydrogen atoms were fixed to 1.2 times the $U$ value of the atoms to which they are linked (1.5 times for methyl groups). One of the 12-crown-4 molecules was modeled as disordered. The site occupancies of the disordered atoms where fixed to 0.5. Chemically equivalent $\mathrm{C}-\mathrm{C}$ and $\mathrm{C}-\mathrm{O}$ bonds for the disordered atoms were restrained to be similar. The disordered atoms thermal parameters were also restrained to behave as a rigid group. Restraints were also applied to "non-disordered" atoms of the other 18-crown-6/12-crown-4 potassium complexes. These sites are likely disordered, and generate a number of checkCIF alerts. However, the weak, twinned data did not strongly support additional parameters. Restraints and constraints were used to obtain a stable model that converged. Atom C310 is likely an average position of two disordered positions. Its close proximity to the crystallographic special position also caused the thermal parameters to be large. Its thermal parameters were subsequently constrained to the value of its neighbor, C320. The program SQUEEZE was used to compensate for the contribution of disordered solvents contained in voids within the crystal lattice from the diffraction intensities. This procedure was applied to the data file and the submitted model is based on the solvent removed data. Based on the total electron density found in the voids $\left(175 \mathrm{e} / \AA^{3}\right)$, it is likely that $\sim 4$ ether molecules are present in the unit cell. See "_platon_squeeze_details" in this .cif for more information. The full numbering scheme of compound 4' can be found in the full details of the X-ray structure determination (CIF), which is included as Supporting Information. 


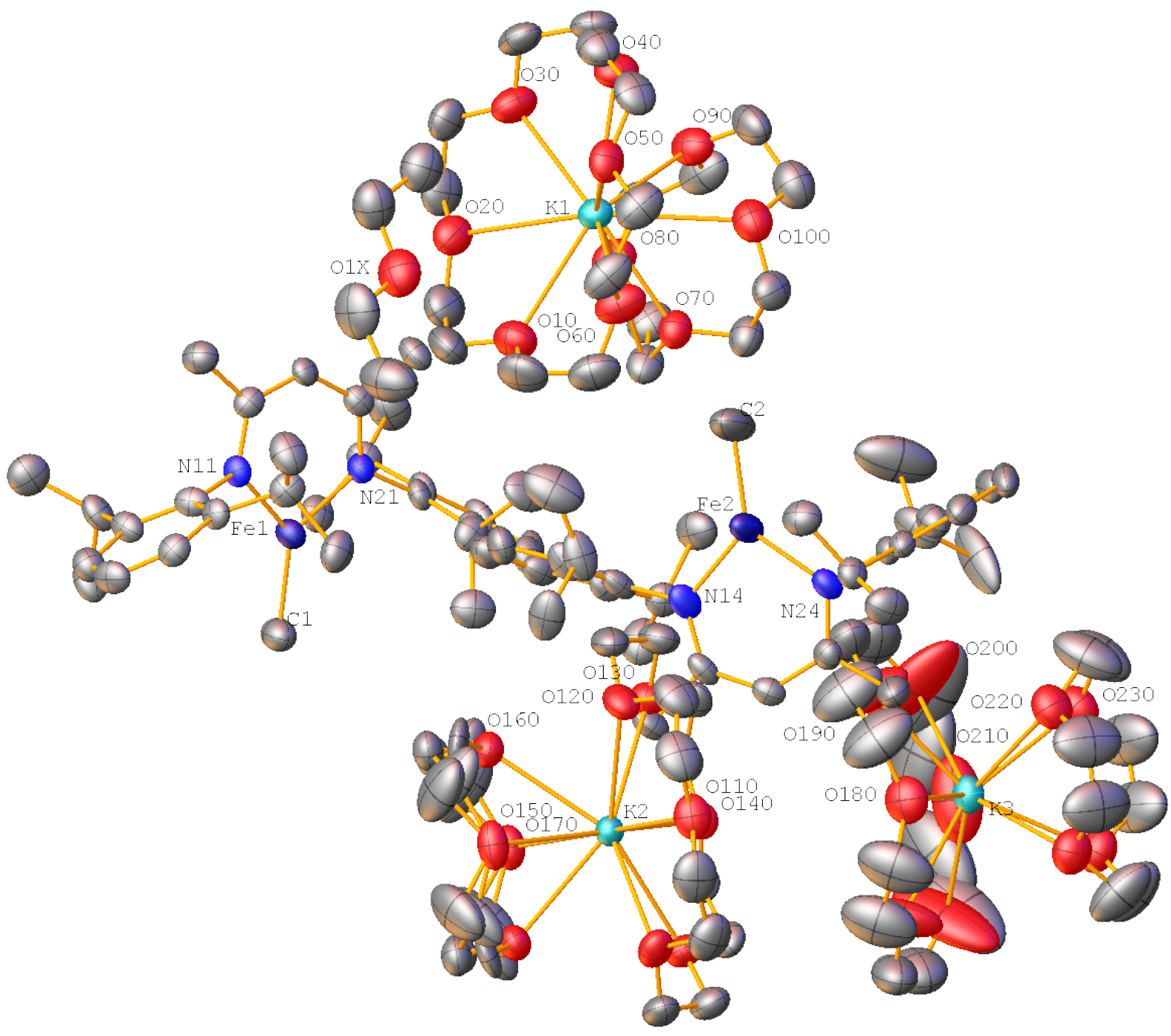

Figure S-27: A partial numbering scheme of 4' with 50\% thermal ellipsoid probability levels. The hydrogen atoms are shown as circles for clarity. 
Table S-19. Crystal data and structure refinement for 4'.

Identification code

CSD Deposition Number

Empirical formula

Formula weight

Temperature

Wavelength

Crystal system

Space group

Unit cell dimensions

Volume

Z

Density (calculated)

Absorption coefficient

$\mathrm{F}(000)$

Crystal size

Crystal color and habit

Diffractometer

Theta range for data collection

Index ranges

Reflections collected

Independent reflections

Observed reflections (I > 2sigma(I))

Completeness to theta $=67.369^{\circ}$

Absorption correction

Max. and min. transmission

Solution method

Refinement method

Data / restraints / parameters

Goodness-of-fit on $F^{2}$

Final R indices [I $>2 \operatorname{sigma}(\mathrm{I})]$

$\mathrm{R}$ indices (all data)

Largest diff. peak and hole
007-16130

1944811

$\mathrm{C}_{52} \mathrm{H}_{89} \mathrm{FeKN}_{2} \mathrm{O}_{10.5}$

1005.20

93(2) K

\section{$1.54184 \AA$}

Monoclinic

$P 21 / \mathrm{m}$

$a=12.8093(4) \AA$

$\alpha=90^{\circ}$.

$b=49.1255(11) \AA$

$\beta=90.131(2)^{\circ}$.

$c=18.9290(4) \AA$

$\gamma=90^{\circ}$.

\section{8}

11911.3(5) $\AA^{3}$

$1.121 \mathrm{~g} / \mathrm{cm}^{3}$

$3.067 \mathrm{~mm}^{-1}$

4352

$0.050 \times 0.050 \times 0.020 \mathrm{~mm}^{3}$

Red Plate

Rigaku Saturn 944+ CCD

1.799 to $67.369^{\circ}$.

$-14<=\mathrm{h}<=14,-58<=\mathrm{k}<=58,0<=1<=22$

21384

$21384[\mathrm{R}(\mathrm{int})=0.1394]$

17694

$98.7 \%$

Semi-empirical from equivalents

1.00000 and 0.62537

SHELXT-2014/5 (Sheldrick, 2014)

SHELXL-2014/7 (Sheldrick, 2014)

21384 / $196 / 1265$

1.119

$\mathrm{R} 1=0.0992, \mathrm{wR} 2=0.2324$

$\mathrm{R} 1=0.1147, \mathrm{wR} 2=0.2420$

0.956 and -0.866 e. $\AA^{-3}$ 
Table S-20. Atomic coordinates ( $\times 10^{4}$ ) and equivalent isotropic displacement parameters $\left(\AA^{2} \times 10^{3}\right)$ for 4'. $U(e q)$ is defined as one third of the trace of the orthogonalized $U^{i j}$ tensor.

\begin{tabular}{|c|c|c|c|c|}
\hline & $\mathrm{x}$ & $\mathrm{y}$ & $\mathrm{z}$ & $\mathrm{U}(\mathrm{eq})$ \\
\hline $\mathrm{Fe}(1)$ & $-1012(1)$ & $3900(1)$ & $4792(1)$ & $34(1)$ \\
\hline $\mathrm{C}(1)$ & $-1314(6)$ & $3513(1)$ & 4498(4) & $57(2)$ \\
\hline $\mathrm{N}(11)$ & $-1864(4)$ & 4169(1) & $5274(2)$ & $31(1)$ \\
\hline $\mathrm{N}(21)$ & $238(4)$ & $4120(1)$ & $4713(2)$ & $32(1)$ \\
\hline $\mathrm{C}(11)$ & $-2327(6)$ & $4630(1)$ & $5679(4)$ & $50(2)$ \\
\hline $\mathrm{C}(21)$ & $-1563(5)$ & $4432(1)$ & $5362(3)$ & $35(1)$ \\
\hline $\mathrm{C}(31)$ & $-573(4)$ & $4524(1)$ & $5175(3)$ & $32(1)$ \\
\hline $\mathrm{C}(41)$ & $286(5)$ & $4385(1)$ & 4897(3) & $34(1)$ \\
\hline$C(51)$ & 1291(5) & $4540(1)$ & $4816(3)$ & $44(2)$ \\
\hline $\mathrm{C}(12)$ & $-2751(5)$ & $4070(1)$ & $5639(3)$ & $37(1)$ \\
\hline $\mathrm{C}(22)$ & $-2657(5)$ & $4003(1)$ & $6374(3)$ & $33(1)$ \\
\hline $\mathrm{C}(32)$ & $-3491(5)$ & $3881(1)$ & $6707(3)$ & $38(1)$ \\
\hline$C(42)$ & $-4385(5)$ & $3811(1)$ & $6350(3)$ & $46(2)$ \\
\hline$C(52)$ & $-4482(6)$ & $3878(1)$ & $5641(3)$ & $45(2)$ \\
\hline$C(62)$ & $-3682(5)$ & $4009(1)$ & $5284(3)$ & $38(1)$ \\
\hline $\mathrm{C}(72)$ & $-1639(6)$ & $4058(1)$ & $6767(3)$ & $44(2)$ \\
\hline $\mathrm{C}(82)$ & $-1031(6)$ & $3789(2)$ & $6852(4)$ & $57(2)$ \\
\hline $\mathrm{C}(92)$ & $-1787(6)$ & 4193(2) & 7481(4) & $61(2)$ \\
\hline$C(102)$ & $-3846(5)$ & $4086(1)$ & $4517(3)$ & $45(2)$ \\
\hline$C(112)$ & $-3950(6)$ & $3833(2)$ & $4052(4)$ & $59(2)$ \\
\hline$C(122)$ & $-4801(7)$ & $4272(2)$ & 4414(4) & $64(2)$ \\
\hline$C(13)$ & $1172(4)$ & $3988(1)$ & $4467(3)$ & $33(1)$ \\
\hline$C(23)$ & $1764(5)$ & $3823(1)$ & $4945(3)$ & $37(1)$ \\
\hline$C(33)$ & 2641(5) & $3693(2)$ & $4675(3)$ & $47(2)$ \\
\hline$C(43)$ & $2940(5)$ & $3718(2)$ & 3999(4) & $47(2)$ \\
\hline$C(53)$ & $2364(5)$ & $3877(1)$ & $3537(3)$ & $42(2)$ \\
\hline$C(63)$ & $1468(5)$ & 4012(1) & $3758(3)$ & $36(1)$ \\
\hline$C(73)$ & $1465(6)$ & $3797(1)$ & $5710(3)$ & $45(2)$ \\
\hline$C(83)$ & $1262(7)$ & $3499(2)$ & 5913(4) & $61(2)$ \\
\hline$C(93)$ & $2246(8)$ & $3930(2)$ & $6196(4)$ & $68(2)$ \\
\hline$C(103)$ & $815(5)$ & $4173(1)$ & $3241(3)$ & $41(2)$ \\
\hline
\end{tabular}




\begin{tabular}{|c|c|c|c|c|}
\hline$C(113)$ & $115(6)$ & $3981(2)$ & 2811(3) & $49(2)$ \\
\hline$C(123)$ & $1476(7)$ & $4352(2)$ & 2751(4) & $68(2)$ \\
\hline $\mathrm{Fe}(2)$ & $4201(1)$ & $3637(1)$ & $10205(1)$ & $52(1)$ \\
\hline $\mathrm{C}(2)$ & $4149(9)$ & $4047(2)$ & $10366(7)$ & $108(4)$ \\
\hline $\mathrm{N}(14)$ & $3240(4)$ & $3385(1)$ & $9770(2)$ & $40(1)$ \\
\hline $\mathrm{N}(24)$ & $5215(4)$ & $3372(1)$ & $10531(2)$ & $36(1)$ \\
\hline$C(14)$ & $2551(6)$ & $2927(1)$ & $9458(4)$ & $51(2)$ \\
\hline $\mathrm{C}(24)$ & $3375(5)$ & $3110(1)$ & $9783(3)$ & $36(1)$ \\
\hline$C(34)$ & $4231(5)$ & $2986(1)$ & 10091(3) & $37(1)$ \\
\hline $\mathrm{C}(44)$ & $5099(5)$ & $3097(1)$ & 10441(3) & $38(1)$ \\
\hline$C(54)$ & $5940(6)$ & 2913(1) & $10730(5)$ & $59(2)$ \\
\hline$C(15)$ & $2386(5)$ & $3492(1)$ & $9378(3)$ & $42(2)$ \\
\hline$C(25)$ & $2441(5)$ & $3497(1)$ & $8623(3)$ & $38(1)$ \\
\hline$C(35)$ & $1586(6)$ & $3597(1)$ & $8259(3)$ & $44(2)$ \\
\hline$C(45)$ & $700(6)$ & $3692(1)$ & $8579(4)$ & $47(2)$ \\
\hline$C(55)$ & $685(5)$ & $3705(2)$ & 9324(4) & $49(2)$ \\
\hline$C(65)$ & $1507(5)$ & $3604(1)$ & $9716(3)$ & $46(2)$ \\
\hline$C(75)$ & $3418(6)$ & $3417(1)$ & $8247(3)$ & $44(2)$ \\
\hline$C(85)$ & $4119(7)$ & $3669(2)$ & $8158(5)$ & $64(2)$ \\
\hline$C(95)$ & $3243(7)$ & $3269(2)$ & $7553(4)$ & $70(2)$ \\
\hline$C(105)$ & $1467(6)$ & $3613(2)$ & $10515(3)$ & $61(2)$ \\
\hline$C(115)$ & $1106(11)$ & $3888(2)$ & $10790(5)$ & $103(4)$ \\
\hline$C(125)$ & $735(6)$ & $3395(2)$ & 10789(4) & $67(2)$ \\
\hline$C(16)$ & $6100(5)$ & $3468(1)$ & $10902(3)$ & $35(1)$ \\
\hline$C(26)$ & 6983(6) & $3562(2)$ & $10545(4)$ & $50(2)$ \\
\hline$C(36)$ & 7803(5) & $3666(1)$ & $10920(4)$ & $53(2)$ \\
\hline$C(46)$ & $7812(6)$ & $3675(2)$ & $11644(5)$ & $56(2)$ \\
\hline$C(56)$ & 6943(6) & $3580(1)$ & 12011(4) & $49(2)$ \\
\hline$C(66)$ & $6082(5)$ & $3479(1)$ & 11651(3) & $35(1)$ \\
\hline$C(76)$ & 6993(7) & $3551(2)$ & $9745(4)$ & $70(2)$ \\
\hline$C(86)$ & 7951(7) & $3397(3)$ & $9475(6)$ & $122(5)$ \\
\hline$C(96)$ & $6917(15)$ & $3834(3)$ & $9410(7)$ & $156(7)$ \\
\hline$C(106)$ & $5112(5)$ & $3389(1)$ & $12046(3)$ & $40(1)$ \\
\hline$C(116)$ & $4401(6)$ & $3629(2)$ & 12213(4) & $56(2)$ \\
\hline$C(126)$ & $5337(7)$ & $3235(2)$ & 12737(4) & $67(2)$ \\
\hline $\mathrm{K}(1)$ & $3340(1)$ & $4968(1)$ & $7858(1)$ & $43(1)$ \\
\hline
\end{tabular}




\begin{tabular}{|c|c|c|c|c|}
\hline $\mathrm{O}(10)$ & $1852(5)$ & $4516(1)$ & $7800(3)$ & $70(2)$ \\
\hline $\mathrm{O}(20)$ & 1398(4) & $4966(1)$ & 6945(3) & $62(1)$ \\
\hline $\mathrm{O}(30)$ & $2599(5)$ & $5444(1)$ & 7021(3) & $70(2)$ \\
\hline $\mathrm{O}(40)$ & $3821(5)$ & $5513(1)$ & $8287(3)$ & $65(2)$ \\
\hline $\mathrm{O}(50)$ & $3116(4)$ & $5155(1)$ & 9351(3) & $59(1)$ \\
\hline $\mathrm{O}(60)$ & $3131(5)$ & $4590(1)$ & $9037(3)$ & $74(2)$ \\
\hline $\mathrm{O}(70)$ & $4389(4)$ & $4473(1)$ & $7662(2)$ & $53(1)$ \\
\hline $\mathrm{O}(80)$ & $4003(5)$ & 4791(1) & $6455(3)$ & $66(1)$ \\
\hline $\mathrm{O}(90)$ & $5203(5)$ & $5185(1)$ & 7201(3) & $66(2)$ \\
\hline $\mathrm{O}(100)$ & $5552(5)$ & $4852(1)$ & $8427(3)$ & $69(2)$ \\
\hline $\mathrm{C}(10)$ & $946(8)$ & $4534(2)$ & $7372(6)$ & $84(3)$ \\
\hline$C(20)$ & $1179(8)$ & $4694(2)$ & $6764(5)$ & $83(3)$ \\
\hline$C(30)$ & $1516(8)$ & $5146(2)$ & 6371(4) & $73(2)$ \\
\hline $\mathrm{C}(40)$ & $1664(7)$ & $5424(2)$ & 6626(4) & $63(2)$ \\
\hline$C(50)$ & $2727(7)$ & $5696(2)$ & $7339(5)$ & $67(2)$ \\
\hline $\mathrm{C}(60)$ & $3763(7)$ & $5707(2)$ & $7717(5)$ & $68(2)$ \\
\hline$C(70)$ & $3378(8)$ & $5609(2)$ & $8916(5)$ & $72(2)$ \\
\hline $\mathrm{C}(80)$ & $3656(7)$ & $5404(2)$ & $9475(4)$ & $68(2)$ \\
\hline $\mathrm{C}(90)$ & $3369(8)$ & $4954(2)$ & 9831(4) & $76(3)$ \\
\hline$C(100)$ & $2782(8)$ & $4701(2)$ & $9674(4)$ & $71(3)$ \\
\hline$C(110)$ & 2649(9) & $4338(2)$ & $8836(5)$ & $81(3)$ \\
\hline$C(120)$ & 1674(9) & $4377(2)$ & $8452(5)$ & $83(3)$ \\
\hline$C(130)$ & $4074(7)$ & $4358(2)$ & 7003(4) & $60(2)$ \\
\hline$C(140)$ & $4445(8)$ & $4527(2)$ & 6391(4) & $69(2)$ \\
\hline$C(150)$ & $4602(8)$ & 4999(2) & $6124(5)$ & $76(3)$ \\
\hline$C(160)$ & $5541(8)$ & $5084(2)$ & $6546(5)$ & $81(3)$ \\
\hline$C(170)$ & 5991(7) & $5234(2)$ & $7708(5)$ & $76(3)$ \\
\hline$C(180)$ & $6397(8)$ & 4974(2) & $8049(5)$ & $84(3)$ \\
\hline$C(190)$ & $5668(7)$ & $4568(2)$ & $8513(4)$ & $66(2)$ \\
\hline$C(200)$ & $5406(6)$ & $4407(2)$ & 7851(4) & $61(2)$ \\
\hline $\mathrm{K}(2)$ & $544(1)$ & 2500 & $12844(1)$ & $36(1)$ \\
\hline $\mathrm{O}(110)$ & $1350(6)$ & 2500 & $14325(3)$ & $59(2)$ \\
\hline $\mathrm{O}(120)$ & $713(4)$ & 2992(1) & $13688(3)$ & $58(1)$ \\
\hline $\mathrm{O}(130)$ & $1605(4)$ & $3005(1)$ & $12325(3)$ & $53(1)$ \\
\hline $\mathrm{O}(140)$ & $2326(5)$ & 2500 & 11834(3) & $45(2)$ \\
\hline $\mathrm{O}(150)$ & $-1527(5)$ & 2500 & $13552(3)$ & $59(2)$ \\
\hline
\end{tabular}




\begin{tabular}{|c|c|c|c|c|}
\hline $\mathrm{O}(160)$ & $-1045(4)$ & $2886(1)$ & $12498(2)$ & $52(1)$ \\
\hline$C(210)$ & $1125(8)$ & $2740(2)$ & $14712(4)$ & $79(3)$ \\
\hline$C(220)$ & 1373(8) & 2981(2) & $14280(5)$ & $77(3)$ \\
\hline$C(230)$ & $801(7)$ & $3236(2)$ & $13285(5)$ & $65(2)$ \\
\hline$C(240)$ & $1728(7)$ & $3227(2)$ & $12811(5)$ & $63(2)$ \\
\hline$C(250)$ & $2464(6)$ & $2979(2)$ & 11881(4) & $56(2)$ \\
\hline$C(260)$ & $2317(6)$ & $2741(2)$ & $11401(4)$ & $57(2)$ \\
\hline $\mathrm{C}(8 \mathrm{X})$ & $-2202(17)$ & $2745(2)$ & $13517(16)$ & $123(10)$ \\
\hline$C(7 Y)$ & $-2068(9)$ & 2911(3) & $12777(8)$ & $54(3)$ \\
\hline$C(6 X)$ & $-1707(12)$ & $2892(3)$ & 11892(7) & $56(3)$ \\
\hline $\mathrm{C}(5 \mathrm{Y})$ & $-1205(19)$ & $2736(2)$ & $11245(11)$ & $86(7)$ \\
\hline $\mathrm{O}(170)$ & $-572(5)$ & 2500 & $11448(3)$ & $56(2)$ \\
\hline $\mathrm{K}(3)$ & $8000(2)$ & 2500 & 7411(1) & $45(1)$ \\
\hline $\mathrm{O}(180)$ & $6620(7)$ & 2500 & $8621(4)$ & $73(2)$ \\
\hline $\mathrm{O}(190)$ & $6619(9)$ & $2950(2)$ & 7694(4) & $131(4)$ \\
\hline $\mathrm{O}(200)$ & $7698(13)$ & $2967(3)$ & $6479(6)$ & $265(9)$ \\
\hline $\mathrm{O}(220)$ & $9078(5)$ & $2788(1)$ & $8573(3)$ & $70(2)$ \\
\hline $\mathrm{O}(230)$ & $9941(5)$ & $2786(1)$ & $7205(3)$ & $68(2)$ \\
\hline$C(310)$ & $6260(14)$ & $2757(2)$ & $8764(7)$ & $144(4)$ \\
\hline$C(320)$ & $6048(14)$ & 2958(3) & $8297(7)$ & $144(4)$ \\
\hline$C(330)$ & $6524(9)$ & $3166(2)$ & $7258(7)$ & $103(3)$ \\
\hline$C(360)$ & $8062(16)$ & 2711(3) & $5446(9)$ & $254(8)$ \\
\hline$C(370)$ & $9358(13)$ & $2630(2)$ & $9132(5)$ & $129(5)$ \\
\hline$C(380)$ & $9811(12)$ & 2972(3) & $8364(6)$ & $126(5)$ \\
\hline$C(390)$ & $10220(11)$ & $2970(3)$ & $7713(6)$ & $115(4)$ \\
\hline $\mathrm{O}(1 \mathrm{X})$ & $-223(5)$ & $4828(1)$ & $9069(3)$ & $80(2)$ \\
\hline$C(3 X)$ & $-418(8)$ & $5110(2)$ & $8910(6)$ & $86(3)$ \\
\hline$C(4 X)$ & $501(8)$ & $5233(2)$ & $8609(5)$ & $82(3)$ \\
\hline$C(1 X)$ & $-789(10)$ & 4411(2) & $9575(7)$ & $109(4)$ \\
\hline$C(2 X)$ & $-1060(8)$ & $4699(2)$ & $9376(6)$ & $93(3)$ \\
\hline$C(400)$ & $10725(10)$ & $2634(2)$ & $6929(8)$ & $144(6)$ \\
\hline $\mathrm{O}(210)$ & $8342(12)$ & 2500 & $5842(7)$ & $167(6)$ \\
\hline$C(340)$ & $7097(12)$ & $3158(3)$ & $6658(7)$ & $125(4)$ \\
\hline$C(350)$ & $8191(16)$ & $2950(4)$ & $5813(7)$ & $229(8)$ \\
\hline$C(5 X)$ & $-1020(20)$ & $2777(4)$ & $11421(10)$ & $87(6)$ \\
\hline $\mathrm{C}(6 \mathrm{Y})$ & $-1053(15)$ & 2934(3) & $11718(6)$ & $63(4)$ \\
\hline
\end{tabular}


$\mathrm{C}(7 \mathrm{X})$

$-1698(11)$

2928(4)

13143(7)

59(3)

$\mathrm{C}(8 \mathrm{Y})$

$-1920(20)$

2778(4)

13411(10)

103(8) 
Table S-21. Bond lengths $[\AA]$ and angles $\left[{ }^{\circ}\right]$ for $\mathbf{4}^{\text {'. }}$

\begin{tabular}{|c|c|}
\hline $\mathrm{Fe}(1)-\mathrm{N}(21)$ & $1.938(5)$ \\
\hline $\mathrm{Fe}(1)-\mathrm{N}(11)$ & $1.942(5)$ \\
\hline $\mathrm{Fe}(1)-\mathrm{C}(1)$ & $2.016(7)$ \\
\hline $\mathrm{C}(1)-\mathrm{H}(1 \mathrm{~A})$ & 0.9800 \\
\hline $\mathrm{C}(1)-\mathrm{H}(1 \mathrm{~B})$ & 0.9800 \\
\hline $\mathrm{C}(1)-\mathrm{H}(1 \mathrm{C})$ & 0.9800 \\
\hline $\mathrm{N}(11)-\mathrm{C}(21)$ & $1.361(7)$ \\
\hline $\mathrm{N}(11)-\mathrm{C}(12)$ & $1.417(7)$ \\
\hline $\mathrm{N}(21)-\mathrm{C}(41)$ & $1.349(7)$ \\
\hline $\mathrm{N}(21)-\mathrm{C}(13)$ & $1.438(7)$ \\
\hline $\mathrm{C}(11)-\mathrm{C}(21)$ & $1.505(9)$ \\
\hline $\mathrm{C}(11)-\mathrm{H}(11 \mathrm{G})$ & 0.9800 \\
\hline $\mathrm{C}(11)-\mathrm{H}(11 \mathrm{H})$ & 0.9800 \\
\hline C(11)-H(11I) & 0.9800 \\
\hline $\mathrm{C}(21)-\mathrm{C}(31)$ & $1.393(8)$ \\
\hline$C(31)-C(41)$ & $1.401(8)$ \\
\hline $\mathrm{C}(31)-\mathrm{H}(31)$ & 0.9500 \\
\hline$C(41)-C(51)$ & $1.503(8)$ \\
\hline $\mathrm{C}(51)-\mathrm{H}(51 \mathrm{~A})$ & 0.9800 \\
\hline $\mathrm{C}(51)-\mathrm{H}(51 \mathrm{~B})$ & 0.9800 \\
\hline $\mathrm{C}(51)-\mathrm{H}(51 \mathrm{C})$ & 0.9800 \\
\hline$C(12)-C(62)$ & $1.399(9)$ \\
\hline$C(12)-C(22)$ & $1.434(8)$ \\
\hline$C(22)-C(32)$ & $1.380(8)$ \\
\hline$C(22)-C(72)$ & $1.523(9)$ \\
\hline$C(32)-C(42)$ & $1.372(10)$ \\
\hline $\mathrm{C}(32)-\mathrm{H}(32)$ & 0.9500 \\
\hline$C(42)-C(52)$ & $1.389(9)$ \\
\hline $\mathrm{C}(42)-\mathrm{H}(42)$ & 0.9500 \\
\hline$C(52)-C(62)$ & $1.386(9)$ \\
\hline $\mathrm{C}(52)-\mathrm{H}(52)$ & 0.9500 \\
\hline$C(62)-C(102)$ & $1.514(9)$ \\
\hline$C(72)-C(92)$ & $1.519(9)$ \\
\hline$C(72)-C(82)$ & $1.543(10)$ \\
\hline
\end{tabular}




\begin{tabular}{|c|c|}
\hline $\mathrm{C}(72)-\mathrm{H}(72)$ & 1.0000 \\
\hline $\mathrm{C}(82)-\mathrm{H}(82 \mathrm{~A})$ & 0.9800 \\
\hline $\mathrm{C}(82)-\mathrm{H}(82 \mathrm{~B})$ & 0.9800 \\
\hline $\mathrm{C}(82)-\mathrm{H}(82 \mathrm{C})$ & 0.9800 \\
\hline $\mathrm{C}(92)-\mathrm{H}(92 \mathrm{~A})$ & 0.9800 \\
\hline $\mathrm{C}(92)-\mathrm{H}(92 \mathrm{~B})$ & 0.9800 \\
\hline $\mathrm{C}(92)-\mathrm{H}(92 \mathrm{C})$ & 0.9800 \\
\hline$C(102)-C(112)$ & $1.529(9)$ \\
\hline$C(102)-C(122)$ & $1.538(11)$ \\
\hline $\mathrm{C}(102)-\mathrm{H}(102)$ & 1.0000 \\
\hline $\mathrm{C}(112)-\mathrm{H}(11 \mathrm{~J})$ & 0.9800 \\
\hline $\mathrm{C}(112)-\mathrm{H}(11 \mathrm{~K})$ & 0.9800 \\
\hline $\mathrm{C}(112)-\mathrm{H}(11 \mathrm{~L})$ & 0.9800 \\
\hline $\mathrm{C}(122)-\mathrm{H}(12 \mathrm{G})$ & 0.9800 \\
\hline $\mathrm{C}(122)-\mathrm{H}(12 \mathrm{H})$ & 0.9800 \\
\hline $\mathrm{C}(122)-\mathrm{H}(12 \mathrm{I})$ & 0.9800 \\
\hline$C(13)-C(63)$ & $1.401(8)$ \\
\hline$C(13)-C(23)$ & $1.433(8)$ \\
\hline$C(23)-C(33)$ & $1.389(9)$ \\
\hline$C(23)-C(73)$ & $1.505(8)$ \\
\hline$C(33)-C(43)$ & $1.343(10)$ \\
\hline $\mathrm{C}(33)-\mathrm{H}(33)$ & 0.9500 \\
\hline$C(43)-C(53)$ & $1.384(10)$ \\
\hline $\mathrm{C}(43)-\mathrm{H}(43)$ & 0.9500 \\
\hline$C(53)-C(63)$ & $1.390(9)$ \\
\hline C(53)-H(53) & 0.9500 \\
\hline $\mathrm{C}(63)-\mathrm{C}(103)$ & $1.508(9)$ \\
\hline$C(73)-C(93)$ & $1.504(10)$ \\
\hline$C(73)-C(83)$ & $1.534(10)$ \\
\hline $\mathrm{C}(73)-\mathrm{H}(73)$ & 1.0000 \\
\hline $\mathrm{C}(83)-\mathrm{H}(83 \mathrm{~A})$ & 0.9800 \\
\hline $\mathrm{C}(83)-\mathrm{H}(83 \mathrm{~B})$ & 0.9800 \\
\hline $\mathrm{C}(83)-\mathrm{H}(83 \mathrm{C})$ & 0.9800 \\
\hline $\mathrm{C}(93)-\mathrm{H}(93 \mathrm{~A})$ & 0.9800 \\
\hline $\mathrm{C}(93)-\mathrm{H}(93 \mathrm{~B})$ & 0.9800 \\
\hline $\mathrm{C}(93)-\mathrm{H}(93 \mathrm{C})$ & 0.9800 \\
\hline
\end{tabular}




\begin{tabular}{|c|c|}
\hline$C(103)-C(113)$ & $1.532(9)$ \\
\hline$C(103)-C(123)$ & $1.536(9)$ \\
\hline $\mathrm{C}(103)-\mathrm{H}(103)$ & 1.0000 \\
\hline $\mathrm{C}(113)-\mathrm{H}(11 \mathrm{M})$ & 0.9800 \\
\hline $\mathrm{C}(113)-\mathrm{H}(11 \mathrm{~N})$ & 0.9800 \\
\hline $\mathrm{C}(113)-\mathrm{H}(11 \mathrm{O})$ & 0.9800 \\
\hline $\mathrm{C}(123)-\mathrm{H}(12 \mathrm{~J})$ & 0.9800 \\
\hline $\mathrm{C}(123)-\mathrm{H}(12 \mathrm{~K})$ & 0.9800 \\
\hline $\mathrm{C}(123)-\mathrm{H}(12 \mathrm{~L})$ & 0.9800 \\
\hline $\mathrm{Fe}(2)-\mathrm{N}(14)$ & $1.929(5)$ \\
\hline $\mathrm{Fe}(2)-\mathrm{N}(24)$ & $1.938(5)$ \\
\hline $\mathrm{Fe}(2)-\mathrm{C}(2)$ & $2.038(9)$ \\
\hline $\mathrm{C}(2)-\mathrm{H}(0 \mathrm{AA})$ & 0.9800 \\
\hline $\mathrm{C}(2)-\mathrm{H}(0 \mathrm{AB})$ & 0.9800 \\
\hline $\mathrm{C}(2)-\mathrm{H}(0 \mathrm{AC})$ & 0.9800 \\
\hline N(14)-C(24) & $1.359(8)$ \\
\hline $\mathrm{N}(14)-\mathrm{C}(15)$ & $1.422(8)$ \\
\hline N(24)-C(44) & $1.370(8)$ \\
\hline $\mathrm{N}(24)-\mathrm{C}(16)$ & $1.414(8)$ \\
\hline$C(14)-C(24)$ & $1.516(8)$ \\
\hline $\mathrm{C}(14)-\mathrm{H}(14 \mathrm{~A})$ & 0.9800 \\
\hline $\mathrm{C}(14)-\mathrm{H}(14 \mathrm{~B})$ & 0.9800 \\
\hline $\mathrm{C}(14)-\mathrm{H}(14 \mathrm{C})$ & 0.9800 \\
\hline$C(24)-C(34)$ & $1.383(9)$ \\
\hline$C(34)-C(44)$ & $1.403(9)$ \\
\hline $\mathrm{C}(34)-\mathrm{H}(34)$ & 0.9500 \\
\hline $\mathrm{C}(44)-\mathrm{C}(54)$ & $1.507(9)$ \\
\hline $\mathrm{C}(54)-\mathrm{H}(54 \mathrm{~A})$ & 0.9800 \\
\hline $\mathrm{C}(54)-\mathrm{H}(54 \mathrm{~B})$ & 0.9800 \\
\hline $\mathrm{C}(54)-\mathrm{H}(54 \mathrm{C})$ & 0.9800 \\
\hline$C(15)-C(65)$ & $1.409(10)$ \\
\hline$C(15)-C(25)$ & $1.431(8)$ \\
\hline$C(25)-C(35)$ & $1.381(9)$ \\
\hline$C(25)-C(75)$ & $1.495(9)$ \\
\hline$C(35)-C(45)$ & $1.370(10)$ \\
\hline $\mathrm{C}(35)-\mathrm{H}(35)$ & 0.9500 \\
\hline
\end{tabular}




\begin{tabular}{|c|c|}
\hline $\mathrm{C}(45)-\mathrm{C}(55)$ & $1.412(10)$ \\
\hline $\mathrm{C}(45)-\mathrm{H}(45)$ & 0.9500 \\
\hline$C(55)-C(65)$ & $1.378(10)$ \\
\hline $\mathrm{C}(55)-\mathrm{H}(55)$ & 0.9500 \\
\hline$C(65)-C(105)$ & $1.515(9)$ \\
\hline$C(75)-C(95)$ & $1.516(10)$ \\
\hline$C(75)-C(85)$ & $1.543(10)$ \\
\hline $\mathrm{C}(75)-\mathrm{H}(75)$ & 1.0000 \\
\hline $\mathrm{C}(85)-\mathrm{H}(85 \mathrm{~A})$ & 0.9800 \\
\hline $\mathrm{C}(85)-\mathrm{H}(85 \mathrm{~B})$ & 0.9800 \\
\hline $\mathrm{C}(85)-\mathrm{H}(85 \mathrm{C})$ & 0.9800 \\
\hline $\mathrm{C}(95)-\mathrm{H}(95 \mathrm{~A})$ & 0.9800 \\
\hline $\mathrm{C}(95)-\mathrm{H}(95 \mathrm{~B})$ & 0.9800 \\
\hline $\mathrm{C}(95)-\mathrm{H}(95 \mathrm{C})$ & 0.9800 \\
\hline$C(105)-C(125)$ & $1.517(11)$ \\
\hline$C(105)-C(115)$ & $1.519(14)$ \\
\hline $\mathrm{C}(105)-\mathrm{H}(105)$ & 1.0000 \\
\hline $\mathrm{C}(115)-\mathrm{H}(11 \mathrm{~A})$ & 0.9800 \\
\hline $\mathrm{C}(115)-\mathrm{H}(11 \mathrm{~B})$ & 0.9800 \\
\hline $\mathrm{C}(115)-\mathrm{H}(11 \mathrm{C})$ & 0.9800 \\
\hline $\mathrm{C}(125)-\mathrm{H}(12 \mathrm{~A})$ & 0.9800 \\
\hline $\mathrm{C}(125)-\mathrm{H}(12 \mathrm{~B})$ & 0.9800 \\
\hline $\mathrm{C}(125)-\mathrm{H}(12 \mathrm{C})$ & 0.9800 \\
\hline$C(16)-C(26)$ & $1.398(9)$ \\
\hline$C(16)-C(66)$ & $1.419(9)$ \\
\hline$C(26)-C(36)$ & $1.367(10)$ \\
\hline$C(26)-C(76)$ & $1.514(11)$ \\
\hline$C(36)-C(46)$ & $1.371(11)$ \\
\hline $\mathrm{C}(36)-\mathrm{H}(36)$ & 0.9500 \\
\hline$C(46)-C(56)$ & $1.394(11)$ \\
\hline $\mathrm{C}(46)-\mathrm{H}(46)$ & 0.9500 \\
\hline$C(56)-C(66)$ & $1.387(9)$ \\
\hline $\mathrm{C}(56)-\mathrm{H}(56)$ & 0.9500 \\
\hline$C(66)-C(106)$ & $1.518(9)$ \\
\hline$C(76)-C(86)$ & $1.530(13)$ \\
\hline$C(76)-C(96)$ & $1.533(15)$ \\
\hline
\end{tabular}




\begin{tabular}{|c|c|}
\hline $\mathrm{C}(76)-\mathrm{H}(76)$ & 1.0000 \\
\hline $\mathrm{C}(86)-\mathrm{H}(86 \mathrm{~A})$ & 0.9800 \\
\hline $\mathrm{C}(86)-\mathrm{H}(86 \mathrm{~B})$ & 0.9800 \\
\hline $\mathrm{C}(86)-\mathrm{H}(86 \mathrm{C})$ & 0.9800 \\
\hline $\mathrm{C}(96)-\mathrm{H}(96 \mathrm{~A})$ & 0.9800 \\
\hline $\mathrm{C}(96)-\mathrm{H}(96 \mathrm{~B})$ & 0.9800 \\
\hline $\mathrm{C}(96)-\mathrm{H}(96 \mathrm{C})$ & 0.9800 \\
\hline$C(106)-C(116)$ & $1.525(9)$ \\
\hline$C(106)-C(126)$ & $1.537(10)$ \\
\hline $\mathrm{C}(106)-\mathrm{H}(106)$ & 1.0000 \\
\hline$C(116)-H(11 D)$ & 0.9800 \\
\hline $\mathrm{C}(116)-\mathrm{H}(11 \mathrm{E})$ & 0.9800 \\
\hline $\mathrm{C}(116)-\mathrm{H}(11 \mathrm{~F})$ & 0.9800 \\
\hline $\mathrm{C}(126)-\mathrm{H}(12 \mathrm{D})$ & 0.9800 \\
\hline$C(126)-H(12 E)$ & 0.9800 \\
\hline $\mathrm{C}(126)-\mathrm{H}(12 \mathrm{~F})$ & 0.9800 \\
\hline $\mathrm{K}(1)-\mathrm{O}(70)$ & $2.802(5)$ \\
\hline $\mathrm{K}(1)-\mathrm{O}(40)$ & $2.866(5)$ \\
\hline $\mathrm{K}(1)-\mathrm{O}(90)$ & $2.897(6)$ \\
\hline $\mathrm{K}(1)-\mathrm{O}(60)$ & $2.915(5)$ \\
\hline $\mathrm{K}(1)-\mathrm{O}(80)$ & $2.920(5)$ \\
\hline $\mathrm{K}(1)-\mathrm{O}(10)$ & $2.926(6)$ \\
\hline $\mathrm{K}(1)-\mathrm{O}(30)$ & $2.981(6)$ \\
\hline $\mathrm{K}(1)-\mathrm{O}(50)$ & $2.986(5)$ \\
\hline $\mathrm{K}(1)-\mathrm{O}(20)$ & $3.025(6)$ \\
\hline $\mathrm{K}(1)-\mathrm{O}(100)$ & $3.082(6)$ \\
\hline K(1)-C(130) & $3.531(7)$ \\
\hline $\mathrm{O}(10)-\mathrm{C}(10)$ & $1.416(11)$ \\
\hline $\mathrm{O}(10)-\mathrm{C}(120)$ & $1.431(10)$ \\
\hline $\mathrm{O}(20)-\mathrm{C}(30)$ & $1.408(10)$ \\
\hline $\mathrm{O}(20)-\mathrm{C}(20)$ & $1.410(11)$ \\
\hline $\mathrm{O}(30)-\mathrm{C}(50)$ & $1.385(10)$ \\
\hline $\mathrm{O}(30)-\mathrm{C}(40)$ & $1.413(10)$ \\
\hline $\mathrm{O}(40)-\mathrm{C}(70)$ & $1.401(10)$ \\
\hline $\mathrm{O}(40)-\mathrm{C}(60)$ & $1.440(9)$ \\
\hline $\mathrm{O}(50)-\mathrm{C}(90)$ & $1.377(10)$ \\
\hline
\end{tabular}




\begin{tabular}{|c|c|}
\hline $\mathrm{O}(50)-\mathrm{C}(80)$ & $1.427(10)$ \\
\hline $\mathrm{O}(60)-\mathrm{C}(100)$ & $1.397(9)$ \\
\hline $\mathrm{O}(60)-\mathrm{C}(110)$ & $1.435(11)$ \\
\hline $\mathrm{O}(70)-\mathrm{C}(200)$ & $1.389(9)$ \\
\hline $\mathrm{O}(70)-\mathrm{C}(130)$ & $1.426(8)$ \\
\hline $\mathrm{O}(80)-\mathrm{C}(140)$ & $1.422(9)$ \\
\hline $\mathrm{O}(80)-\mathrm{C}(150)$ & $1.423(10)$ \\
\hline $\mathrm{O}(90)-\mathrm{C}(160)$ & $1.405(10)$ \\
\hline $\mathrm{O}(90)-\mathrm{C}(170)$ & $1.413(11)$ \\
\hline $\mathrm{O}(100)-\mathrm{C}(190)$ & $1.415(10)$ \\
\hline $\mathrm{O}(100)-\mathrm{C}(180)$ & $1.431(11)$ \\
\hline$C(10)-C(20)$ & $1.427(14)$ \\
\hline $\mathrm{C}(10)-\mathrm{H}(10 \mathrm{~A})$ & 0.9900 \\
\hline $\mathrm{C}(10)-\mathrm{H}(10 \mathrm{~B})$ & 0.9900 \\
\hline $\mathrm{C}(20)-\mathrm{H}(20 \mathrm{~A})$ & 0.9900 \\
\hline $\mathrm{C}(20)-\mathrm{H}(20 \mathrm{~B})$ & 0.9900 \\
\hline$C(30)-C(40)$ & $1.460(12)$ \\
\hline $\mathrm{C}(30)-\mathrm{H}(30 \mathrm{~A})$ & 0.9900 \\
\hline $\mathrm{C}(30)-\mathrm{H}(30 \mathrm{~B})$ & 0.9900 \\
\hline $\mathrm{C}(40)-\mathrm{H}(40 \mathrm{C})$ & 0.9900 \\
\hline C(40)-H(40D) & 0.9900 \\
\hline$C(50)-C(60)$ & $1.507(12)$ \\
\hline $\mathrm{C}(50)-\mathrm{H}(50 \mathrm{~A})$ & 0.9900 \\
\hline $\mathrm{C}(50)-\mathrm{H}(50 \mathrm{~B})$ & 0.9900 \\
\hline $\mathrm{C}(60)-\mathrm{H}(60 \mathrm{~A})$ & 0.9900 \\
\hline $\mathrm{C}(60)-\mathrm{H}(60 \mathrm{~B})$ & 0.9900 \\
\hline $\mathrm{C}(70)-\mathrm{C}(80)$ & $1.502(12)$ \\
\hline $\mathrm{C}(70)-\mathrm{H}(70 \mathrm{~A})$ & 0.9900 \\
\hline $\mathrm{C}(70)-\mathrm{H}(70 \mathrm{~B})$ & 0.9900 \\
\hline $\mathrm{C}(80)-\mathrm{H}(80 \mathrm{~A})$ & 0.9900 \\
\hline $\mathrm{C}(80)-\mathrm{H}(80 \mathrm{~B})$ & 0.9900 \\
\hline $\mathrm{C}(90)-\mathrm{C}(100)$ & $1.485(13)$ \\
\hline $\mathrm{C}(90)-\mathrm{H}(90 \mathrm{~A})$ & 0.9900 \\
\hline $\mathrm{C}(90)-\mathrm{H}(90 \mathrm{~B})$ & 0.9900 \\
\hline $\mathrm{C}(100)-\mathrm{H}(10 \mathrm{C})$ & 0.9900 \\
\hline $\mathrm{C}(100)-\mathrm{H}(10 \mathrm{D})$ & 0.9900 \\
\hline
\end{tabular}




\begin{tabular}{|c|c|}
\hline$C(110)-C(120)$ & $1.457(14)$ \\
\hline $\mathrm{C}(110)-\mathrm{H}(11 \mathrm{P})$ & 0.9900 \\
\hline $\mathrm{C}(110)-\mathrm{H}(11 \mathrm{Q})$ & 0.9900 \\
\hline $\mathrm{C}(120)-\mathrm{H}(12 \mathrm{M})$ & 0.9900 \\
\hline $\mathrm{C}(120)-\mathrm{H}(12 \mathrm{~N})$ & 0.9900 \\
\hline$C(130)-C(140)$ & $1.502(11)$ \\
\hline $\mathrm{C}(130)-\mathrm{H}(13 \mathrm{~A})$ & 0.9900 \\
\hline $\mathrm{C}(130)-\mathrm{H}(13 \mathrm{~B})$ & 0.9900 \\
\hline $\mathrm{C}(140)-\mathrm{H}(14 \mathrm{D})$ & 0.9900 \\
\hline $\mathrm{C}(140)-\mathrm{H}(14 \mathrm{E})$ & 0.9900 \\
\hline$C(150)-C(160)$ & $1.501(14)$ \\
\hline $\mathrm{C}(150)-\mathrm{H}(15 \mathrm{~A})$ & 0.9900 \\
\hline $\mathrm{C}(150)-\mathrm{H}(15 \mathrm{~B})$ & 0.9900 \\
\hline $\mathrm{C}(160)-\mathrm{H}(16 \mathrm{~A})$ & 0.9900 \\
\hline $\mathrm{C}(160)-\mathrm{H}(16 \mathrm{~B})$ & 0.9900 \\
\hline$C(170)-C(180)$ & $1.521(14)$ \\
\hline $\mathrm{C}(170)-\mathrm{H}(17 \mathrm{~A})$ & 0.9900 \\
\hline $\mathrm{C}(170)-\mathrm{H}(17 \mathrm{~B})$ & 0.9900 \\
\hline $\mathrm{C}(180)-\mathrm{H}(18 \mathrm{~A})$ & 0.9900 \\
\hline $\mathrm{C}(180)-\mathrm{H}(18 \mathrm{~B})$ & 0.9900 \\
\hline$C(190)-C(200)$ & $1.519(11)$ \\
\hline C(190)-H(19A) & 0.9900 \\
\hline $\mathrm{C}(190)-\mathrm{H}(19 \mathrm{~B})$ & 0.9900 \\
\hline $\mathrm{C}(200)-\mathrm{H}(20 \mathrm{C})$ & 0.9900 \\
\hline $\mathrm{C}(200)-\mathrm{H}(20 \mathrm{D})$ & 0.9900 \\
\hline $\mathrm{K}(2)-\mathrm{O}(160)$ & $2.854(5)$ \\
\hline $\mathrm{K}(2)-\mathrm{O}(160) \# 1$ & $2.854(5)$ \\
\hline $\mathrm{K}(2)-\mathrm{O}(120) \# 1$ & $2.904(5)$ \\
\hline $\mathrm{K}(2)-\mathrm{O}(120)$ & $2.904(5)$ \\
\hline $\mathrm{K}(2)-\mathrm{O}(150)$ & $2.974(7)$ \\
\hline $\mathrm{K}(2)-\mathrm{O}(140)$ & $2.981(6)$ \\
\hline $\mathrm{K}(2)-\mathrm{O}(110)$ & $2.986(7)$ \\
\hline $\mathrm{K}(2)-\mathrm{O}(130) \# 1$ & $2.996(5)$ \\
\hline $\mathrm{K}(2)-\mathrm{O}(130)$ & $2.996(5)$ \\
\hline $\mathrm{K}(2)-\mathrm{O}(170)$ & $3.001(7)$ \\
\hline $\mathrm{O}(110)-\mathrm{C}(210)$ & $1.419(9)$ \\
\hline
\end{tabular}




\begin{tabular}{|c|c|}
\hline $\mathrm{O}(110)-\mathrm{C}(210) \# 1$ & $1.419(9)$ \\
\hline $\mathrm{O}(120)-\mathrm{C}(220)$ & $1.402(10)$ \\
\hline $\mathrm{O}(120)-\mathrm{C}(230)$ & $1.426(9)$ \\
\hline $\mathrm{O}(130)-\mathrm{C}(250)$ & $1.392(9)$ \\
\hline $\mathrm{O}(130)-\mathrm{C}(240)$ & $1.437(9)$ \\
\hline $\mathrm{O}(140)-\mathrm{C}(260)$ & $1.439(8)$ \\
\hline $\mathrm{O}(140)-\mathrm{C}(260) \# 1$ & $1.439(8)$ \\
\hline $\mathrm{O}(150)-\mathrm{C}(8 \mathrm{Y}) \# 1$ & $1.481(13)$ \\
\hline $\mathrm{O}(150)-\mathrm{C}(8 \mathrm{Y})$ & $1.481(13)$ \\
\hline $\mathrm{O}(150)-\mathrm{C}(8 \mathrm{X}) \# 1$ & $1.482(13)$ \\
\hline $\mathrm{O}(150)-\mathrm{C}(8 \mathrm{X})$ & $1.482(13)$ \\
\hline $\mathrm{O}(160)-\mathrm{C}(7 \mathrm{Y})$ & $1.420(11)$ \\
\hline $\mathrm{O}(160)-\mathrm{C}(6 \mathrm{X})$ & $1.427(11)$ \\
\hline $\mathrm{O}(160)-\mathrm{C}(7 \mathrm{X})$ & $1.495(11)$ \\
\hline $\mathrm{O}(160)-\mathrm{C}(6 \mathrm{Y})$ & $1.496(11)$ \\
\hline$C(210)-C(220)$ & $1.473(13)$ \\
\hline $\mathrm{C}(210)-\mathrm{H}(21 \mathrm{~A})$ & 0.9900 \\
\hline $\mathrm{C}(210)-\mathrm{H}(21 \mathrm{~B})$ & 0.9900 \\
\hline $\mathrm{C}(220)-\mathrm{H}(22 \mathrm{~A})$ & 0.9900 \\
\hline $\mathrm{C}(220)-\mathrm{H}(22 \mathrm{~B})$ & 0.9900 \\
\hline$C(230)-C(240)$ & $1.490(12)$ \\
\hline $\mathrm{C}(230)-\mathrm{H}(23 \mathrm{~A})$ & 0.9900 \\
\hline $\mathrm{C}(230)-\mathrm{H}(23 \mathrm{~B})$ & 0.9900 \\
\hline $\mathrm{C}(240)-\mathrm{H}(24 \mathrm{~A})$ & 0.9900 \\
\hline $\mathrm{C}(240)-\mathrm{H}(24 \mathrm{~B})$ & 0.9900 \\
\hline$C(250)-C(260)$ & $1.492(11)$ \\
\hline $\mathrm{C}(250)-\mathrm{H}(25 \mathrm{~A})$ & 0.9900 \\
\hline $\mathrm{C}(250)-\mathrm{H}(25 \mathrm{~B})$ & 0.9900 \\
\hline $\mathrm{C}(260)-\mathrm{H}(26 \mathrm{~A})$ & 0.9900 \\
\hline $\mathrm{C}(260)-\mathrm{H}(26 \mathrm{~B})$ & 0.9900 \\
\hline $\mathrm{C}(8 \mathrm{X})-\mathrm{C}(7 \mathrm{X})$ & $1.316(15)$ \\
\hline $\mathrm{C}(8 \mathrm{X})-\mathrm{H}(8 \mathrm{XA})$ & 0.9900 \\
\hline $\mathrm{C}(8 \mathrm{X})-\mathrm{H}(8 \mathrm{XB})$ & 0.9900 \\
\hline C(7Y)-C(8Y) & $1.376(15)$ \\
\hline $\mathrm{C}(7 \mathrm{Y})-\mathrm{H}(7 \mathrm{YA})$ & 0.9900 \\
\hline $\mathrm{C}(7 \mathrm{Y})-\mathrm{H}(7 \mathrm{YB})$ & 0.9900 \\
\hline
\end{tabular}




\begin{tabular}{|c|c|}
\hline$C(6 X)-C(5 X)$ & $1.378(16)$ \\
\hline $\mathrm{C}(6 \mathrm{X})-\mathrm{H}(6 \mathrm{XA})$ & 0.9900 \\
\hline $\mathrm{C}(6 \mathrm{X})-\mathrm{H}(6 \mathrm{XB})$ & 0.9900 \\
\hline $\mathrm{C}(5 \mathrm{Y})-\mathrm{C}(6 \mathrm{Y})$ & $1.336(15)$ \\
\hline $\mathrm{C}(5 \mathrm{Y})-\mathrm{O}(170)$ & $1.466(12)$ \\
\hline $\mathrm{C}(5 \mathrm{Y})-\mathrm{H}(5 \mathrm{YA})$ & 0.9900 \\
\hline $\mathrm{C}(5 \mathrm{Y})-\mathrm{H}(5 \mathrm{YB})$ & 0.9900 \\
\hline $\mathrm{O}(170)-\mathrm{C}(5 \mathrm{Y}) \# 1$ & $1.466(12)$ \\
\hline $\mathrm{O}(170)-\mathrm{C}(5 \mathrm{X})$ & $1.477(12)$ \\
\hline $\mathrm{O}(170)-\mathrm{C}(5 \mathrm{X}) \# 1$ & $1.477(13)$ \\
\hline $\mathrm{K}(3)-\mathrm{O}(230) \# 1$ & $2.883(6)$ \\
\hline $\mathrm{K}(3)-\mathrm{O}(230)$ & $2.883(6)$ \\
\hline $\mathrm{K}(3)-\mathrm{O}(190)$ & $2.884(7)$ \\
\hline $\mathrm{K}(3)-\mathrm{O}(190) \# 1$ & $2.884(7)$ \\
\hline $\mathrm{K}(3)-\mathrm{O}(180)$ & $2.896(8)$ \\
\hline $\mathrm{K}(3)-\mathrm{O}(200) \# 1$ & $2.919(9)$ \\
\hline $\mathrm{K}(3)-\mathrm{O}(200)$ & $2.919(9)$ \\
\hline $\mathrm{K}(3)-\mathrm{O}(220)$ & $2.956(6)$ \\
\hline $\mathrm{K}(3)-\mathrm{O}(220) \# 1$ & $2.956(6)$ \\
\hline $\mathrm{K}(3)-\mathrm{O}(210)$ & $3.003(13)$ \\
\hline $\mathrm{O}(180)-\mathrm{C}(310) \# 1$ & $1.371(10)$ \\
\hline $\mathrm{O}(180)-\mathrm{C}(310)$ & $1.371(10)$ \\
\hline $\mathrm{O}(190)-\mathrm{C}(330)$ & $1.348(10)$ \\
\hline $\mathrm{O}(190)-\mathrm{C}(320)$ & $1.357(14)$ \\
\hline $\mathrm{O}(200)-\mathrm{C}(340)$ & $1.262(10)$ \\
\hline $\mathrm{O}(200)-\mathrm{C}(350)$ & $1.414(11)$ \\
\hline $\mathrm{O}(220)-\mathrm{C}(370)$ & $1.361(12)$ \\
\hline $\mathrm{O}(220)-\mathrm{C}(380)$ & $1.364(12)$ \\
\hline $\mathrm{O}(230)-\mathrm{C}(400)$ & $1.356(12)$ \\
\hline $\mathrm{O}(230)-\mathrm{C}(390)$ & $1.369(11)$ \\
\hline $\mathrm{C}(310)-\mathrm{C}(320)$ & $1.352(13)$ \\
\hline $\mathrm{C}(310)-\mathrm{H}(31 \mathrm{~A})$ & 0.9900 \\
\hline $\mathrm{C}(310)-\mathrm{H}(31 \mathrm{~B})$ & 0.9900 \\
\hline $\mathrm{C}(320)-\mathrm{H}(32 \mathrm{~A})$ & 0.9900 \\
\hline $\mathrm{C}(320)-\mathrm{H}(32 \mathrm{~B})$ & 0.9900 \\
\hline$C(330)-C(340)$ & $1.356(13)$ \\
\hline
\end{tabular}




\begin{tabular}{|c|c|}
\hline $\mathrm{C}(330)-\mathrm{H}(33 \mathrm{~A})$ & 0.9900 \\
\hline $\mathrm{C}(330)-\mathrm{H}(33 \mathrm{~B})$ & 0.9900 \\
\hline $\mathrm{C}(360)-\mathrm{O}(210)$ & $1.326(13)$ \\
\hline$C(360)-C(350)$ & $1.377(17)$ \\
\hline $\mathrm{C}(360)-\mathrm{H}(36 \mathrm{~A})$ & 0.9900 \\
\hline $\mathrm{C}(360)-\mathrm{H}(36 \mathrm{~B})$ & 0.9900 \\
\hline $\mathrm{C}(370)-\mathrm{C}(370) \# 1$ & $1.28(2)$ \\
\hline $\mathrm{C}(370)-\mathrm{H}(37 \mathrm{~A})$ & 0.9900 \\
\hline $\mathrm{C}(370)-\mathrm{H}(37 \mathrm{~B})$ & 0.9900 \\
\hline $\mathrm{C}(380)-\mathrm{C}(390)$ & $1.340(14)$ \\
\hline $\mathrm{C}(380)-\mathrm{H}(38 \mathrm{~A})$ & 0.9900 \\
\hline $\mathrm{C}(380)-\mathrm{H}(38 \mathrm{~B})$ & 0.9900 \\
\hline $\mathrm{C}(390)-\mathrm{H}(39 \mathrm{~A})$ & 0.9900 \\
\hline $\mathrm{C}(390)-\mathrm{H}(39 \mathrm{~B})$ & 0.9900 \\
\hline $\mathrm{O}(1 \mathrm{X})-\mathrm{C}(2 \mathrm{X})$ & $1.375(11)$ \\
\hline $\mathrm{O}(1 \mathrm{X})-\mathrm{C}(3 \mathrm{X})$ & $1.439(12)$ \\
\hline$C(3 X)-C(4 X)$ & $1.444(13)$ \\
\hline $\mathrm{C}(3 \mathrm{X})-\mathrm{H}(3 \mathrm{XA})$ & 0.9900 \\
\hline $\mathrm{C}(3 \mathrm{X})-\mathrm{H}(3 \mathrm{XB})$ & 0.9900 \\
\hline $\mathrm{C}(4 \mathrm{X})-\mathrm{H}(4 \mathrm{XA})$ & 0.9800 \\
\hline $\mathrm{C}(4 \mathrm{X})-\mathrm{H}(4 \mathrm{XB})$ & 0.9800 \\
\hline $\mathrm{C}(4 \mathrm{X})-\mathrm{H}(4 \mathrm{XC})$ & 0.9800 \\
\hline$C(1 X)-C(2 X)$ & $1.500(15)$ \\
\hline $\mathrm{C}(1 \mathrm{X})-\mathrm{H}(1 \mathrm{XA})$ & 0.9800 \\
\hline $\mathrm{C}(1 \mathrm{X})-\mathrm{H}(1 \mathrm{XB})$ & 0.9800 \\
\hline $\mathrm{C}(1 \mathrm{X})-\mathrm{H}(1 \mathrm{XC})$ & 0.9800 \\
\hline $\mathrm{C}(2 \mathrm{X})-\mathrm{H}(2 \mathrm{XA})$ & 0.9900 \\
\hline $\mathrm{C}(2 \mathrm{X})-\mathrm{H}(2 \mathrm{XB})$ & 0.9900 \\
\hline $\mathrm{C}(400)-\mathrm{C}(400) \# 1$ & $1.32(2)$ \\
\hline $\mathrm{C}(400)-\mathrm{H}(40 \mathrm{~A})$ & 0.9900 \\
\hline $\mathrm{C}(400)-\mathrm{H}(40 \mathrm{~B})$ & 0.9900 \\
\hline $\mathrm{O}(210)-\mathrm{C}(360) \# 1$ & $1.326(13)$ \\
\hline $\mathrm{C}(340)-\mathrm{H}(34 \mathrm{~A})$ & 0.9900 \\
\hline $\mathrm{C}(340)-\mathrm{H}(34 \mathrm{~B})$ & 0.9900 \\
\hline $\mathrm{C}(350)-\mathrm{H}(35 \mathrm{~A})$ & 0.9900 \\
\hline $\mathrm{C}(350)-\mathrm{H}(35 \mathrm{~B})$ & 0.9900 \\
\hline
\end{tabular}




\begin{tabular}{|c|c|}
\hline $\mathrm{C}(5 \mathrm{X})-\mathrm{H}(5 \mathrm{XA})$ & 0.9900 \\
\hline $\mathrm{C}(5 \mathrm{X})-\mathrm{H}(5 \mathrm{XB})$ & 0.9900 \\
\hline $\mathrm{C}(6 \mathrm{Y})-\mathrm{H}(6 \mathrm{YA})$ & 0.9900 \\
\hline C(6Y)-H(6YB) & 0.9900 \\
\hline $\mathrm{C}(7 \mathrm{X})-\mathrm{H}(7 \mathrm{XA})$ & 0.9900 \\
\hline $\mathrm{C}(7 \mathrm{X})-\mathrm{H}(7 \mathrm{XB})$ & 0.9900 \\
\hline C(8Y)-H(8YA) & 0.9900 \\
\hline C(8Y)-H(8YB) & 0.9900 \\
\hline $\mathrm{N}(21)-\mathrm{Fe}(1)-\mathrm{N}(11)$ & $97.0(2)$ \\
\hline $\mathrm{N}(21)-\mathrm{Fe}(1)-\mathrm{C}(1)$ & $131.5(3)$ \\
\hline $\mathrm{N}(11)-\mathrm{Fe}(1)-\mathrm{C}(1)$ & $131.5(3)$ \\
\hline $\mathrm{Fe}(1)-\mathrm{C}(1)-\mathrm{H}(1 \mathrm{~A})$ & 109.5 \\
\hline $\mathrm{Fe}(1)-\mathrm{C}(1)-\mathrm{H}(1 \mathrm{~B})$ & 109.5 \\
\hline $\mathrm{H}(1 \mathrm{~A})-\mathrm{C}(1)-\mathrm{H}(1 \mathrm{~B})$ & 109.5 \\
\hline $\mathrm{Fe}(1)-\mathrm{C}(1)-\mathrm{H}(1 \mathrm{C})$ & 109.5 \\
\hline $\mathrm{H}(1 \mathrm{~A})-\mathrm{C}(1)-\mathrm{H}(1 \mathrm{C})$ & 109.5 \\
\hline $\mathrm{H}(1 \mathrm{~B})-\mathrm{C}(1)-\mathrm{H}(1 \mathrm{C})$ & 109.5 \\
\hline $\mathrm{C}(21)-\mathrm{N}(11)-\mathrm{C}(12)$ & $119.6(5)$ \\
\hline $\mathrm{C}(21)-\mathrm{N}(11)-\mathrm{Fe}(1)$ & $123.1(4)$ \\
\hline $\mathrm{C}(12)-\mathrm{N}(11)-\mathrm{Fe}(1)$ & $116.6(4)$ \\
\hline $\mathrm{C}(41)-\mathrm{N}(21)-\mathrm{C}(13)$ & $118.6(5)$ \\
\hline $\mathrm{C}(41)-\mathrm{N}(21)-\mathrm{Fe}(1)$ & $123.8(4)$ \\
\hline $\mathrm{C}(13)-\mathrm{N}(21)-\mathrm{Fe}(1)$ & $117.5(4)$ \\
\hline $\mathrm{C}(21)-\mathrm{C}(11)-\mathrm{H}(11 \mathrm{G})$ & 109.5 \\
\hline $\mathrm{C}(21)-\mathrm{C}(11)-\mathrm{H}(11 \mathrm{H})$ & 109.5 \\
\hline $\mathrm{H}(11 \mathrm{G})-\mathrm{C}(11)-\mathrm{H}(11 \mathrm{H})$ & 109.5 \\
\hline $\mathrm{C}(21)-\mathrm{C}(11)-\mathrm{H}(11 \mathrm{I})$ & 109.5 \\
\hline $\mathrm{H}(11 \mathrm{G})-\mathrm{C}(11)-\mathrm{H}(11 \mathrm{I})$ & 109.5 \\
\hline $\mathrm{H}(11 \mathrm{H})-\mathrm{C}(11)-\mathrm{H}(11 \mathrm{I})$ & 109.5 \\
\hline $\mathrm{N}(11)-\mathrm{C}(21)-\mathrm{C}(31)$ & $122.4(5)$ \\
\hline $\mathrm{N}(11)-\mathrm{C}(21)-\mathrm{C}(11)$ & $118.6(5)$ \\
\hline $\mathrm{C}(31)-\mathrm{C}(21)-\mathrm{C}(11)$ & $119.0(5)$ \\
\hline $\mathrm{C}(21)-\mathrm{C}(31)-\mathrm{C}(41)$ & $130.8(5)$ \\
\hline $\mathrm{C}(21)-\mathrm{C}(31)-\mathrm{H}(31)$ & 114.6 \\
\hline $\mathrm{C}(41)-\mathrm{C}(31)-\mathrm{H}(31)$ & 114.6 \\
\hline
\end{tabular}




$\begin{array}{ll}\mathrm{N}(21)-\mathrm{C}(41)-\mathrm{C}(31) & 122.2(5) \\ \mathrm{N}(21)-\mathrm{C}(41)-\mathrm{C}(51) & 120.1(5) \\ \mathrm{C}(31)-\mathrm{C}(41)-\mathrm{C}(51) & 117.6(5) \\ \mathrm{C}(41)-\mathrm{C}(51)-\mathrm{H}(51 \mathrm{~A}) & 109.5 \\ \mathrm{C}(41)-\mathrm{C}(51)-\mathrm{H}(51 \mathrm{~B}) & 109.5 \\ \mathrm{H}(51 \mathrm{~A})-\mathrm{C}(51)-\mathrm{H}(51 \mathrm{~B}) & 109.5 \\ \mathrm{C}(41)-\mathrm{C}(51)-\mathrm{H}(51 \mathrm{C}) & 109.5 \\ \mathrm{H}(51 \mathrm{~A})-\mathrm{C}(51)-\mathrm{H}(51 \mathrm{C}) & 109.5 \\ \mathrm{H}(51 \mathrm{~B})-\mathrm{C}(51)-\mathrm{H}(51 \mathrm{C}) & 109.5 \\ \mathrm{C}(62)-\mathrm{C}(12)-\mathrm{N}(11) & 121.5(5) \\ \mathrm{C}(62)-\mathrm{C}(12)-\mathrm{C}(22) & 119.2(5) \\ \mathrm{N}(11)-\mathrm{C}(12)-\mathrm{C}(22) & 119.0(6) \\ \mathrm{C}(32)-\mathrm{C}(22)-\mathrm{C}(12) & 118.6(6) \\ \mathrm{C}(32)-\mathrm{C}(22)-\mathrm{C}(72) & 121.1(5) \\ \mathrm{C}(12)-\mathrm{C}(22)-\mathrm{C}(72) & 120.3(5) \\ \mathrm{C}(42)-\mathrm{C}(32)-\mathrm{C}(22) & 122.0(6) \\ \mathrm{C}(42)-\mathrm{C}(32)-\mathrm{H}(32) & 119.0 \\ \mathrm{C}(22)-\mathrm{C}(32)-\mathrm{H}(32) & 119.0 \\ \mathrm{C}(32)-\mathrm{C}(42)-\mathrm{C}(52) & 119.3(6) \\ \mathrm{C}(32)-\mathrm{C}(42)-\mathrm{H}(42) & 120.4 \\ \mathrm{C}(52)-\mathrm{C}(42)-\mathrm{H}(42) & 120.4 \\ \mathrm{C}(62)-\mathrm{C}(52)-\mathrm{C}(42) & 121.1(6) \\ \mathrm{C}(62)-\mathrm{C}(52)-\mathrm{H}(52) & 119.4 \\ \mathrm{C}(42)-\mathrm{C}(52)-\mathrm{H}(52) & 119.4 \\ \mathrm{C}(52)-\mathrm{C}(62)-\mathrm{C}(12) & 119.7(6) \\ \mathrm{C}(52)-\mathrm{C}(62)-\mathrm{C}(102) & 118.8(6) \\ \mathrm{C}(12)-\mathrm{C}(62)-\mathrm{C}(102) & 121.5(5) \\ \mathrm{C}(92)-\mathrm{C}(72)-\mathrm{C}(22) & 113.8(6) \\ \mathrm{C}(92)-\mathrm{C}(72)-\mathrm{C}(82) & 110.2(6) \\ \mathrm{C}(22)-\mathrm{C}(72)-\mathrm{C}(82) & 109.3(6) \\ \mathrm{C}(92)-\mathrm{C}(72)-\mathrm{H}(72) & 107.7 \\ \mathrm{C}(22)-\mathrm{C}(72)-\mathrm{H}(72) & 107.7 \\ \mathrm{C}(82)-\mathrm{C}(72)-\mathrm{H}(72) & 107.7 \\ \mathrm{C}(82)-\mathrm{H}(82 \mathrm{~A}(82)-\mathrm{H}(82 \mathrm{~B}) & 109.5 \\ & \end{array}$




\begin{tabular}{ll}
$\mathrm{C}(72)-\mathrm{C}(82)-\mathrm{H}(82 \mathrm{C})$ & 109.5 \\
$\mathrm{H}(82 \mathrm{~A})-\mathrm{C}(82)-\mathrm{H}(82 \mathrm{C})$ & 109.5 \\
$\mathrm{H}(82 \mathrm{~B})-\mathrm{C}(82)-\mathrm{H}(82 \mathrm{C})$ & 109.5 \\
$\mathrm{C}(72)-\mathrm{C}(92)-\mathrm{H}(92 \mathrm{~A})$ & 109.5 \\
$\mathrm{C}(72)-\mathrm{C}(92)-\mathrm{H}(92 \mathrm{~B})$ & 109.5 \\
$\mathrm{H}(92 \mathrm{~A})-\mathrm{C}(92)-\mathrm{H}(92 \mathrm{~B})$ & 109.5 \\
$\mathrm{C}(72)-\mathrm{C}(92)-\mathrm{H}(92 \mathrm{C})$ & 109.5 \\
$\mathrm{H}(92 \mathrm{~A})-\mathrm{C}(92)-\mathrm{H}(92 \mathrm{C})$ & 109.5 \\
$\mathrm{H}(92 \mathrm{~B})-\mathrm{C}(92)-\mathrm{H}(92 \mathrm{C})$ & 109.5 \\
$\mathrm{C}(62)-\mathrm{C}(102)-\mathrm{C}(112)$ & $111.1(6)$ \\
$\mathrm{C}(62)-\mathrm{C}(102)-\mathrm{C}(122)$ & $112.2(6)$ \\
$\mathrm{C}(112)-\mathrm{C}(102)-\mathrm{C}(122)$ & $109.9(6)$ \\
$\mathrm{C}(62)-\mathrm{C}(102)-\mathrm{H}(102)$ & 107.8 \\
$\mathrm{C}(112)-\mathrm{C}(102)-\mathrm{H}(102)$ & 107.8 \\
$\mathrm{C}(122)-\mathrm{C}(102)-\mathrm{H}(102)$ & 107.8 \\
$\mathrm{C}(102)-\mathrm{C}(112)-\mathrm{H}(11 \mathrm{~J})$ & 109.5 \\
$\mathrm{C}(102)-\mathrm{C}(112)-\mathrm{H}(11 \mathrm{~K})$ & 109.5 \\
$\mathrm{H}(11 \mathrm{~J})-\mathrm{C}(112)-\mathrm{H}(11 \mathrm{~K})$ & 109.5 \\
$\mathrm{C}(102)-\mathrm{C}(112)-\mathrm{H}(11 \mathrm{~L})$ & 109.5 \\
$\mathrm{H}(11 \mathrm{~J})-\mathrm{C}(112)-\mathrm{H}(11 \mathrm{~L})$ & 109.5 \\
$\mathrm{H}(11 \mathrm{~K})-\mathrm{C}(112)-\mathrm{H}(11 \mathrm{~L})$ & 109.5 \\
$\mathrm{C}(102)-\mathrm{C}(122)-\mathrm{H}(12 \mathrm{G})$ & 109.5 \\
$\mathrm{C}(102)-\mathrm{C}(122)-\mathrm{H}(12 \mathrm{H})$ & 109.5 \\
$\mathrm{H}(12 \mathrm{G})-\mathrm{C}(122)-\mathrm{H}(12 \mathrm{H})$ & 109.5 \\
$\mathrm{C}(102)-\mathrm{C}(122)-\mathrm{H}(12 \mathrm{I})$ & 109.5 \\
$\mathrm{H}(12 \mathrm{G})-\mathrm{C}(122)-\mathrm{H}(12 \mathrm{I})$ & 109.5 \\
$\mathrm{H}(12 \mathrm{H})-\mathrm{C}(122)-\mathrm{H}(12 \mathrm{I})$ & 109.5 \\
$\mathrm{C}(63)-\mathrm{C}(13)-\mathrm{C}(23)$ & $120.5(5)$ \\
$\mathrm{C}(63)-\mathrm{C}(13)-\mathrm{N}(21)$ & $120.0(5)$ \\
$\mathrm{C}(23)-\mathrm{C}(13)-\mathrm{N}(21)$ & $119.4(5)$ \\
$\mathrm{C}(33)-\mathrm{C}(23)-\mathrm{C}(13)$ & $117.2(5)$ \\
$\mathrm{C}(23)-\mathrm{C}(73)$ & $121.5(6)$ \\
& $121.3(6)$ \\
& $122.7(7)$ \\
$\mathrm{C}(33)-\mathrm{C}(73)$ & 118.7 \\
& 118.7 \\
\hline
\end{tabular}




$\begin{array}{ll}\mathrm{C}(33)-\mathrm{C}(43)-\mathrm{C}(53) & 120.1(6) \\ \mathrm{C}(33)-\mathrm{C}(43)-\mathrm{H}(43) & 119.9 \\ \mathrm{C}(53)-\mathrm{C}(43)-\mathrm{H}(43) & 119.9 \\ \mathrm{C}(43)-\mathrm{C}(53)-\mathrm{C}(63) & 121.2(6) \\ \mathrm{C}(43)-\mathrm{C}(53)-\mathrm{H}(53) & 119.4 \\ \mathrm{C}(63)-\mathrm{C}(53)-\mathrm{H}(53) & 119.4 \\ \mathrm{C}(53)-\mathrm{C}(63)-\mathrm{C}(13) & 118.3(6) \\ \mathrm{C}(53)-\mathrm{C}(63)-\mathrm{C}(103) & 120.7(5) \\ \mathrm{C}(13)-\mathrm{C}(63)-\mathrm{C}(103) & 121.0(5) \\ \mathrm{C}(93)-\mathrm{C}(73)-\mathrm{C}(23) & 112.5(6) \\ \mathrm{C}(93)-\mathrm{C}(73)-\mathrm{C}(83) & 111.9(6) \\ \mathrm{C}(23)-\mathrm{C}(73)-\mathrm{C}(83) & 111.3(5) \\ \mathrm{C}(93)-\mathrm{C}(73)-\mathrm{H}(73) & 106.9 \\ \mathrm{C}(23)-\mathrm{C}(73)-\mathrm{H}(73) & 106.9 \\ \mathrm{C}(83)-\mathrm{C}(73)-\mathrm{H}(73) & 106.9 \\ \mathrm{C}(73)-\mathrm{C}(83)-\mathrm{H}(83 \mathrm{~A}) & 109.5 \\ \mathrm{C}(73)-\mathrm{C}(83)-\mathrm{H}(83 \mathrm{~B}) & 109.5 \\ \mathrm{H}(83 \mathrm{~A})-\mathrm{C}(83)-\mathrm{H}(83 \mathrm{~B}) & 109.5 \\ \mathrm{C}(73)-\mathrm{C}(83)-\mathrm{H}(83 \mathrm{C}) & 109.5 \\ \mathrm{H}(83 \mathrm{~A})-\mathrm{C}(83)-\mathrm{H}(83 \mathrm{C}) & 109.5 \\ \mathrm{H}(83 \mathrm{~B})-\mathrm{C}(83)-\mathrm{H}(83 \mathrm{C}) & 109.5 \\ \mathrm{C}(73)-\mathrm{C}(93)-\mathrm{H}(93 \mathrm{~A}) & 109.5 \\ \mathrm{C}(73)-\mathrm{C}(93)-\mathrm{H}(93 \mathrm{~B}) & 109.5 \\ \mathrm{H}(93 \mathrm{~A})-\mathrm{C}(93)-\mathrm{H}(93 \mathrm{~B}) & 109.5 \\ \mathrm{C}(73)-\mathrm{C}(93)-\mathrm{H}(93 \mathrm{C}) & 109.5 \\ \mathrm{H}(93 \mathrm{~A})-\mathrm{C}(93)-\mathrm{H}(93 \mathrm{C}) & 109.5 \\ \mathrm{H}(93 \mathrm{~B})-\mathrm{C}(93)-\mathrm{H}(93 \mathrm{C}) & 109.5 \\ \mathrm{C}(63)-\mathrm{C}(103)-\mathrm{C}(113) & 110.4(5) \\ \mathrm{C}(63)-\mathrm{C}(103)-\mathrm{C}(123) & 112.7(6) \\ \mathrm{C}(113)-\mathrm{C}(103)-\mathrm{C}(123) & 110.8(6) \\ \mathrm{C}(63)-\mathrm{C}(103)-\mathrm{H}(103) & 107.6 \\ \mathrm{C}(113)-\mathrm{C}(103)-\mathrm{H}(103) & 107.6 \\ \mathrm{C}(123)-\mathrm{C}(103)-\mathrm{H}(103) & 107.6 \\ & 109.5 \\ \mathrm{C}(113)-\mathrm{H}(11 \mathrm{M}) & 109.5 \\ & 109.5 \\ & \end{array}$




\begin{tabular}{|c|c|}
\hline $\mathrm{C}(103)-\mathrm{C}(113)-\mathrm{H}(11 \mathrm{O})$ & 109.5 \\
\hline $\mathrm{H}(11 \mathrm{M})-\mathrm{C}(113)-\mathrm{H}(11 \mathrm{O})$ & 109.5 \\
\hline $\mathrm{H}(11 \mathrm{~N})-\mathrm{C}(113)-\mathrm{H}(11 \mathrm{O})$ & 109.5 \\
\hline $\mathrm{C}(103)-\mathrm{C}(123)-\mathrm{H}(12 \mathrm{~J})$ & 109.5 \\
\hline $\mathrm{C}(103)-\mathrm{C}(123)-\mathrm{H}(12 \mathrm{~K})$ & 109.5 \\
\hline $\mathrm{H}(12 \mathrm{~J})-\mathrm{C}(123)-\mathrm{H}(12 \mathrm{~K})$ & 109.5 \\
\hline $\mathrm{C}(103)-\mathrm{C}(123)-\mathrm{H}(12 \mathrm{~L})$ & 109.5 \\
\hline $\mathrm{H}(12 \mathrm{~J})-\mathrm{C}(123)-\mathrm{H}(12 \mathrm{~L})$ & 109.5 \\
\hline $\mathrm{H}(12 \mathrm{~K})-\mathrm{C}(123)-\mathrm{H}(12 \mathrm{~L})$ & 109.5 \\
\hline $\mathrm{N}(14)-\mathrm{Fe}(2)-\mathrm{N}(24)$ & $97.5(2)$ \\
\hline $\mathrm{N}(14)-\mathrm{Fe}(2)-\mathrm{C}(2)$ & $132.7(4)$ \\
\hline $\mathrm{N}(24)-\mathrm{Fe}(2)-\mathrm{C}(2)$ & $129.6(3)$ \\
\hline $\mathrm{Fe}(2)-\mathrm{C}(2)-\mathrm{H}(0 \mathrm{AA})$ & 109.5 \\
\hline $\mathrm{Fe}(2)-\mathrm{C}(2)-\mathrm{H}(0 \mathrm{AB})$ & 109.5 \\
\hline $\mathrm{H}(0 \mathrm{AA})-\mathrm{C}(2)-\mathrm{H}(0 \mathrm{AB})$ & 109.5 \\
\hline $\mathrm{Fe}(2)-\mathrm{C}(2)-\mathrm{H}(0 \mathrm{AC})$ & 109.5 \\
\hline $\mathrm{H}(0 \mathrm{AA})-\mathrm{C}(2)-\mathrm{H}(0 \mathrm{AC})$ & 109.5 \\
\hline $\mathrm{H}(0 \mathrm{AB})-\mathrm{C}(2)-\mathrm{H}(0 \mathrm{AC})$ & 109.5 \\
\hline $\mathrm{C}(24)-\mathrm{N}(14)-\mathrm{C}(15)$ & $118.3(5)$ \\
\hline $\mathrm{C}(24)-\mathrm{N}(14)-\mathrm{Fe}(2)$ & $123.3(4)$ \\
\hline $\mathrm{C}(15)-\mathrm{N}(14)-\mathrm{Fe}(2)$ & $118.4(4)$ \\
\hline $\mathrm{C}(44)-\mathrm{N}(24)-\mathrm{C}(16)$ & $118.6(5)$ \\
\hline $\mathrm{C}(44)-\mathrm{N}(24)-\mathrm{Fe}(2)$ & $123.4(4)$ \\
\hline $\mathrm{C}(16)-\mathrm{N}(24)-\mathrm{Fe}(2)$ & $118.0(4)$ \\
\hline $\mathrm{C}(24)-\mathrm{C}(14)-\mathrm{H}(14 \mathrm{~A})$ & 109.5 \\
\hline $\mathrm{C}(24)-\mathrm{C}(14)-\mathrm{H}(14 \mathrm{~B})$ & 109.5 \\
\hline $\mathrm{H}(14 \mathrm{~A})-\mathrm{C}(14)-\mathrm{H}(14 \mathrm{~B})$ & 109.5 \\
\hline $\mathrm{C}(24)-\mathrm{C}(14)-\mathrm{H}(14 \mathrm{C})$ & 109.5 \\
\hline $\mathrm{H}(14 \mathrm{~A})-\mathrm{C}(14)-\mathrm{H}(14 \mathrm{C})$ & 109.5 \\
\hline $\mathrm{H}(14 \mathrm{~B})-\mathrm{C}(14)-\mathrm{H}(14 \mathrm{C})$ & 109.5 \\
\hline $\mathrm{N}(14)-\mathrm{C}(24)-\mathrm{C}(34)$ & $123.1(5)$ \\
\hline $\mathrm{N}(14)-\mathrm{C}(24)-\mathrm{C}(14)$ & $119.5(6)$ \\
\hline$C(34)-C(24)-C(14)$ & $117.4(6)$ \\
\hline $\mathrm{C}(24)-\mathrm{C}(34)-\mathrm{C}(44)$ & $130.9(6)$ \\
\hline $\mathrm{C}(24)-\mathrm{C}(34)-\mathrm{H}(34)$ & 114.5 \\
\hline $\mathrm{C}(44)-\mathrm{C}(34)-\mathrm{H}(34)$ & 114.5 \\
\hline
\end{tabular}




\begin{tabular}{|c|c|}
\hline $\mathrm{N}(24)-\mathrm{C}(44)-\mathrm{C}(34)$ & $121.8(6)$ \\
\hline $\mathrm{N}(24)-\mathrm{C}(44)-\mathrm{C}(54)$ & $118.0(6)$ \\
\hline $\mathrm{C}(34)-\mathrm{C}(44)-\mathrm{C}(54)$ & $120.2(6)$ \\
\hline $\mathrm{C}(44)-\mathrm{C}(54)-\mathrm{H}(54 \mathrm{~A})$ & 109.5 \\
\hline $\mathrm{C}(44)-\mathrm{C}(54)-\mathrm{H}(54 \mathrm{~B})$ & 109.5 \\
\hline $\mathrm{H}(54 \mathrm{~A})-\mathrm{C}(54)-\mathrm{H}(54 \mathrm{~B})$ & 109.5 \\
\hline $\mathrm{C}(44)-\mathrm{C}(54)-\mathrm{H}(54 \mathrm{C})$ & 109.5 \\
\hline $\mathrm{H}(54 \mathrm{~A})-\mathrm{C}(54)-\mathrm{H}(54 \mathrm{C})$ & 109.5 \\
\hline $\mathrm{H}(54 \mathrm{~B})-\mathrm{C}(54)-\mathrm{H}(54 \mathrm{C})$ & 109.5 \\
\hline $\mathrm{C}(65)-\mathrm{C}(15)-\mathrm{N}(14)$ & $121.5(6)$ \\
\hline$C(65)-C(15)-C(25)$ & $119.1(6)$ \\
\hline $\mathrm{N}(14)-\mathrm{C}(15)-\mathrm{C}(25)$ & $119.3(6)$ \\
\hline $\mathrm{C}(35)-\mathrm{C}(25)-\mathrm{C}(15)$ & $117.6(6)$ \\
\hline$C(35)-C(25)-C(75)$ & $121.3(5)$ \\
\hline$C(15)-C(25)-C(75)$ & $120.9(6)$ \\
\hline$C(45)-C(35)-C(25)$ & $123.9(6)$ \\
\hline $\mathrm{C}(45)-\mathrm{C}(35)-\mathrm{H}(35)$ & 118.1 \\
\hline $\mathrm{C}(25)-\mathrm{C}(35)-\mathrm{H}(35)$ & 118.1 \\
\hline$C(35)-C(45)-C(55)$ & $117.9(7)$ \\
\hline $\mathrm{C}(35)-\mathrm{C}(45)-\mathrm{H}(45)$ & 121.0 \\
\hline $\mathrm{C}(55)-\mathrm{C}(45)-\mathrm{H}(45)$ & 121.0 \\
\hline $\mathrm{C}(65)-\mathrm{C}(55)-\mathrm{C}(45)$ & $120.7(7)$ \\
\hline $\mathrm{C}(65)-\mathrm{C}(55)-\mathrm{H}(55)$ & 119.6 \\
\hline $\mathrm{C}(45)-\mathrm{C}(55)-\mathrm{H}(55)$ & 119.6 \\
\hline $\mathrm{C}(55)-\mathrm{C}(65)-\mathrm{C}(15)$ & $120.4(6)$ \\
\hline$C(55)-C(65)-C(105)$ & $120.0(7)$ \\
\hline$C(15)-C(65)-C(105)$ & $119.6(7)$ \\
\hline$C(25)-C(75)-C(95)$ & $114.7(6)$ \\
\hline$C(25)-C(75)-C(85)$ & $109.1(6)$ \\
\hline$C(95)-C(75)-C(85)$ & $112.0(6)$ \\
\hline $\mathrm{C}(25)-\mathrm{C}(75)-\mathrm{H}(75)$ & 106.9 \\
\hline $\mathrm{C}(95)-\mathrm{C}(75)-\mathrm{H}(75)$ & 106.9 \\
\hline $\mathrm{C}(85)-\mathrm{C}(75)-\mathrm{H}(75)$ & 106.9 \\
\hline $\mathrm{C}(75)-\mathrm{C}(85)-\mathrm{H}(85 \mathrm{~A})$ & 109.5 \\
\hline $\mathrm{C}(75)-\mathrm{C}(85)-\mathrm{H}(85 \mathrm{~B})$ & 109.5 \\
\hline $\mathrm{H}(85 \mathrm{~A})-\mathrm{C}(85)-\mathrm{H}(85 \mathrm{~B})$ & 109.5 \\
\hline
\end{tabular}




$\begin{array}{ll}\mathrm{C}(75)-\mathrm{C}(85)-\mathrm{H}(85 \mathrm{C}) & 109.5 \\ \mathrm{H}(85 \mathrm{~A})-\mathrm{C}(85)-\mathrm{H}(85 \mathrm{C}) & 109.5 \\ \mathrm{H}(85 \mathrm{~B})-\mathrm{C}(85)-\mathrm{H}(85 \mathrm{C}) & 109.5 \\ \mathrm{C}(75)-\mathrm{C}(95)-\mathrm{H}(95 \mathrm{~A}) & 109.5 \\ \mathrm{C}(75)-\mathrm{C}(95)-\mathrm{H}(95 \mathrm{~B}) & 109.5 \\ \mathrm{H}(95 \mathrm{~A})-\mathrm{C}(95)-\mathrm{H}(95 \mathrm{~B}) & 109.5 \\ \mathrm{C}(75)-\mathrm{C}(95)-\mathrm{H}(95 \mathrm{C}) & 109.5 \\ \mathrm{H}(95 \mathrm{~A})-\mathrm{C}(95)-\mathrm{H}(95 \mathrm{C}) & 109.5 \\ \mathrm{H}(95 \mathrm{~B})-\mathrm{C}(95)-\mathrm{H}(95 \mathrm{C}) & 109.5 \\ \mathrm{C}(65)-\mathrm{C}(105)-\mathrm{C}(125) & 110.0(7) \\ \mathrm{C}(65)-\mathrm{C}(105)-\mathrm{C}(115) & 112.3(7) \\ \mathrm{C}(125)-\mathrm{C}(105)-\mathrm{C}(115) & 108.7(7) \\ \mathrm{C}(65)-\mathrm{C}(105)-\mathrm{H}(105) & 108.6 \\ \mathrm{C}(125)-\mathrm{C}(105)-\mathrm{H}(105) & 108.6 \\ \mathrm{C}(115)-\mathrm{C}(105)-\mathrm{H}(105) & 108.6 \\ \mathrm{C}(105)-\mathrm{C}(115)-\mathrm{H}(11 \mathrm{~A}) & 109.5 \\ \mathrm{C}(105)-\mathrm{C}(115)-\mathrm{H}(11 \mathrm{~B}) & 109.5 \\ \mathrm{H}(11 \mathrm{~A})-\mathrm{C}(115)-\mathrm{H}(11 \mathrm{~B}) & 109.5 \\ \mathrm{C}(105)-\mathrm{C}(115)-\mathrm{H}(11 \mathrm{C}) & 109.5 \\ \mathrm{H}(11 \mathrm{~A})-\mathrm{C}(115)-\mathrm{H}(11 \mathrm{C}) & 109.5 \\ \mathrm{H}(11 \mathrm{~B})-\mathrm{C}(115)-\mathrm{H}(11 \mathrm{C}) & 109.5 \\ \mathrm{C}(105)-\mathrm{C}(125)-\mathrm{H}(12 \mathrm{~A}) & 109.5 \\ \mathrm{C}(105)-\mathrm{C}(125)-\mathrm{H}(12 \mathrm{~B}) & 109.5 \\ \mathrm{H}(12 \mathrm{~A})-\mathrm{C}(125)-\mathrm{H}(12 \mathrm{~B}) & 109.5 \\ \mathrm{C}(105)-\mathrm{C}(125)-\mathrm{H}(12 \mathrm{C}) & 109.5 \\ \mathrm{H}(12 \mathrm{~A})-\mathrm{C}(125)-\mathrm{H}(12 \mathrm{C}) & 109.5 \\ \mathrm{H}(12 \mathrm{~B})-\mathrm{C}(125)-\mathrm{H}(12 \mathrm{C}) & 109.5 \\ \mathrm{C}(26)-\mathrm{C}(16)-\mathrm{N}(24) & 121.2(6) \\ \mathrm{C}(26)-\mathrm{C}(16)-\mathrm{C}(66) & 119.1(6) \\ \mathrm{N}(24)-\mathrm{C}(16)-\mathrm{C}(66) & 119.7(5) \\ \mathrm{C}(36)-\mathrm{C}(26)-\mathrm{C}(16) & 119.5(7) \\ \mathrm{C}(36)-\mathrm{C}(26)-\mathrm{C}(76) & 121.7(7) \\ \mathrm{C}(36) & 118.7(7) \\ \mathrm{C}(16) & 118.8 \\ & \end{array}$




$\begin{array}{ll}\mathrm{C}(36)-\mathrm{C}(46)-\mathrm{C}(56) & 118.9(7) \\ \mathrm{C}(36)-\mathrm{C}(46)-\mathrm{H}(46) & 120.6 \\ \mathrm{C}(56)-\mathrm{C}(46)-\mathrm{H}(46) & 120.6 \\ \mathrm{C}(66)-\mathrm{C}(56)-\mathrm{C}(46) & 120.7(7) \\ \mathrm{C}(66)-\mathrm{C}(56)-\mathrm{H}(56) & 119.7 \\ \mathrm{C}(46)-\mathrm{C}(56)-\mathrm{H}(56) & 119.7 \\ \mathrm{C}(56)-\mathrm{C}(66)-\mathrm{C}(16) & 119.3(6) \\ \mathrm{C}(56)-\mathrm{C}(66)-\mathrm{C}(106) & 120.9(6) \\ \mathrm{C}(16)-\mathrm{C}(66)-\mathrm{C}(106) & 119.7(5) \\ \mathrm{C}(26)-\mathrm{C}(76)-\mathrm{C}(86) & 111.1(9) \\ \mathrm{C}(26)-\mathrm{C}(76)-\mathrm{C}(96) & 112.3(8) \\ \mathrm{C}(86)-\mathrm{C}(76)-\mathrm{C}(96) & 111.1(10) \\ \mathrm{C}(26)-\mathrm{C}(76)-\mathrm{H}(76) & 107.4 \\ \mathrm{C}(86)-\mathrm{C}(76)-\mathrm{H}(76) & 107.4 \\ \mathrm{C}(96)-\mathrm{C}(76)-\mathrm{H}(76) & 107.4 \\ \mathrm{C}(76)-\mathrm{C}(86)-\mathrm{H}(86 \mathrm{~A}) & 109.5 \\ \mathrm{C}(76)-\mathrm{C}(86)-\mathrm{H}(86 \mathrm{~B}) & 109.5 \\ \mathrm{H}(86 \mathrm{~A})-\mathrm{C}(86)-\mathrm{H}(86 \mathrm{~B}) & 109.5 \\ \mathrm{C}(76)-\mathrm{C}(86)-\mathrm{H}(86 \mathrm{C}) & 109.5 \\ \mathrm{H}(86 \mathrm{~A})-\mathrm{C}(86)-\mathrm{H}(86 \mathrm{C}) & 109.5 \\ \mathrm{H}(86 \mathrm{~B})-\mathrm{C}(86)-\mathrm{H}(86 \mathrm{C}) & 109.5 \\ \mathrm{C}(76)-\mathrm{C}(96)-\mathrm{H}(96 \mathrm{~A}) & 109.5 \\ \mathrm{C}(76)-\mathrm{C}(96)-\mathrm{H}(96 \mathrm{~B}) & 109.5 \\ \mathrm{H}(96 \mathrm{~A})-\mathrm{C}(96)-\mathrm{H}(96 \mathrm{~B}) & 109.5 \\ \mathrm{C}(76)-\mathrm{C}(96)-\mathrm{H}(96 \mathrm{C}) & 109.5 \\ \mathrm{H}(96 \mathrm{~A})-\mathrm{C}(96)-\mathrm{H}(96 \mathrm{C}) & 109.5 \\ \mathrm{H}(96 \mathrm{~B})-\mathrm{C}(96)-\mathrm{H}(96 \mathrm{C}) & 109.5 \\ \mathrm{C}(66)-\mathrm{C}(106)-\mathrm{C}(116) & 111.4(5) \\ \mathrm{C}(66)-\mathrm{C}(106)-\mathrm{C}(126) & 114.3(6) \\ \mathrm{C}(116)-\mathrm{C}(106)-\mathrm{C}(126) & 108.4(6) \\ \mathrm{C}(66)-\mathrm{C}(106)-\mathrm{H}(106) & 107.5 \\ \mathrm{C}(116)-\mathrm{C}(106)-\mathrm{H}(106) & 107.5 \\ \mathrm{C}(126)-\mathrm{C}(106)-\mathrm{H}(106) & 107.5 \\ \mathrm{C}(116)-\mathrm{H}(11 \mathrm{D}) & 109.5 \\ & 109.5 \\ & \\ \mathrm{C}(116)-\mathrm{H}(11 \mathrm{E}) & 109.5 \\ & \end{array}$




\begin{tabular}{|c|c|}
\hline $\mathrm{C}(106)-\mathrm{C}(116)-\mathrm{H}(11 \mathrm{~F})$ & 109.5 \\
\hline $\mathrm{H}(11 \mathrm{D})-\mathrm{C}(116)-\mathrm{H}(11 \mathrm{~F})$ & 109.5 \\
\hline $\mathrm{H}(11 \mathrm{E})-\mathrm{C}(116)-\mathrm{H}(11 \mathrm{~F})$ & 109.5 \\
\hline $\mathrm{C}(106)-\mathrm{C}(126)-\mathrm{H}(12 \mathrm{D})$ & 109.5 \\
\hline $\mathrm{C}(106)-\mathrm{C}(126)-\mathrm{H}(12 \mathrm{E})$ & 109.5 \\
\hline $\mathrm{H}(12 \mathrm{D})-\mathrm{C}(126)-\mathrm{H}(12 \mathrm{E})$ & 109.5 \\
\hline $\mathrm{C}(106)-\mathrm{C}(126)-\mathrm{H}(12 \mathrm{~F})$ & 109.5 \\
\hline $\mathrm{H}(12 \mathrm{D})-\mathrm{C}(126)-\mathrm{H}(12 \mathrm{~F})$ & 109.5 \\
\hline $\mathrm{H}(12 \mathrm{E})-\mathrm{C}(126)-\mathrm{H}(12 \mathrm{~F})$ & 109.5 \\
\hline $\mathrm{O}(70)-\mathrm{K}(1)-\mathrm{O}(40)$ & $138.17(17)$ \\
\hline $\mathrm{O}(70)-\mathrm{K}(1)-\mathrm{O}(90)$ & $82.38(15)$ \\
\hline $\mathrm{O}(40)-\mathrm{K}(1)-\mathrm{O}(90)$ & $66.44(16)$ \\
\hline $\mathrm{O}(70)-\mathrm{K}(1)-\mathrm{O}(60)$ & $66.07(15)$ \\
\hline $\mathrm{O}(40)-\mathrm{K}(1)-\mathrm{O}(60)$ & $113.40(17)$ \\
\hline $\mathrm{O}(90)-\mathrm{K}(1)-\mathrm{O}(60)$ & $129.82(19)$ \\
\hline $\mathrm{O}(70)-\mathrm{K}(1)-\mathrm{O}(80)$ & $58.80(14)$ \\
\hline $\mathrm{O}(40)-\mathrm{K}(1)-\mathrm{O}(80)$ & $118.19(17)$ \\
\hline $\mathrm{O}(90)-\mathrm{K}(1)-\mathrm{O}(80)$ & $58.52(17)$ \\
\hline $\mathrm{O}(60)-\mathrm{K}(1)-\mathrm{O}(80)$ & $122.33(16)$ \\
\hline $\mathrm{O}(70)-\mathrm{K}(1)-\mathrm{O}(10)$ & $69.53(16)$ \\
\hline $\mathrm{O}(40)-\mathrm{K}(1)-\mathrm{O}(10)$ & $149.16(18)$ \\
\hline $\mathrm{O}(90)-\mathrm{K}(1)-\mathrm{O}(10)$ & $143.17(16)$ \\
\hline $\mathrm{O}(60)-\mathrm{K}(1)-\mathrm{O}(10)$ & $59.05(18)$ \\
\hline $\mathrm{O}(80)-\mathrm{K}(1)-\mathrm{O}(10)$ & $86.09(17)$ \\
\hline $\mathrm{O}(70)-\mathrm{K}(1)-\mathrm{O}(30)$ & $139.75(16)$ \\
\hline $\mathrm{O}(40)-\mathrm{K}(1)-\mathrm{O}(30)$ & $58.97(16)$ \\
\hline $\mathrm{O}(90)-\mathrm{K}(1)-\mathrm{O}(30)$ & $75.14(17)$ \\
\hline $\mathrm{O}(60)-\mathrm{K}(1)-\mathrm{O}(30)$ & $151.29(18)$ \\
\hline $\mathrm{O}(80)-\mathrm{K}(1)-\mathrm{O}(30)$ & $80.97(16)$ \\
\hline $\mathrm{O}(10)-\mathrm{K}(1)-\mathrm{O}(30)$ & 111.66(18) \\
\hline $\mathrm{O}(70)-\mathrm{K}(1)-\mathrm{O}(50)$ & $116.03(15)$ \\
\hline $\mathrm{O}(40)-\mathrm{K}(1)-\mathrm{O}(50)$ & $57.66(16)$ \\
\hline $\mathrm{O}(90)-\mathrm{K}(1)-\mathrm{O}(50)$ & 111.94(17) \\
\hline $\mathrm{O}(60)-\mathrm{K}(1)-\mathrm{O}(50)$ & $57.42(16)$ \\
\hline $\mathrm{O}(80)-\mathrm{K}(1)-\mathrm{O}(50)$ & $168.59(17)$ \\
\hline $\mathrm{O}(10)-\mathrm{K}(1)-\mathrm{O}(50)$ & $101.81(16)$ \\
\hline
\end{tabular}




\begin{tabular}{|c|c|}
\hline $\mathrm{O}(30)-\mathrm{K}(1)-\mathrm{O}(50)$ & $103.31(15)$ \\
\hline $\mathrm{O}(70)-\mathrm{K}(1)-\mathrm{O}(20)$ & $108.47(15)$ \\
\hline $\mathrm{O}(40)-\mathrm{K}(1)-\mathrm{O}(20)$ & $109.88(16)$ \\
\hline $\mathrm{O}(90)-\mathrm{K}(1)-\mathrm{O}(20)$ & $115.63(17)$ \\
\hline $\mathrm{O}(60)-\mathrm{K}(1)-\mathrm{O}(20)$ & $111.06(18)$ \\
\hline $\mathrm{O}(80)-\mathrm{K}(1)-\mathrm{O}(20)$ & $73.79(16)$ \\
\hline $\mathrm{O}(10)-\mathrm{K}(1)-\mathrm{O}(20)$ & $56.11(16)$ \\
\hline $\mathrm{O}(30)-\mathrm{K}(1)-\mathrm{O}(20)$ & $55.81(16)$ \\
\hline $\mathrm{O}(50)-\mathrm{K}(1)-\mathrm{O}(20)$ & $117.43(15)$ \\
\hline $\mathrm{O}(70)-\mathrm{K}(1)-\mathrm{O}(100)$ & $56.34(15)$ \\
\hline $\mathrm{O}(40)-\mathrm{K}(1)-\mathrm{O}(100)$ & $82.86(17)$ \\
\hline $\mathrm{O}(90)-\mathrm{K}(1)-\mathrm{O}(100)$ & $57.34(17)$ \\
\hline $\mathrm{O}(60)-\mathrm{K}(1)-\mathrm{O}(100)$ & $72.61(18)$ \\
\hline $\mathrm{O}(80)-\mathrm{K}(1)-\mathrm{O}(100)$ & $89.67(16)$ \\
\hline $\mathrm{O}(10)-\mathrm{K}(1)-\mathrm{O}(100)$ & $118.16(17)$ \\
\hline $\mathrm{O}(30)-\mathrm{K}(1)-\mathrm{O}(100)$ & $128.42(18)$ \\
\hline $\mathrm{O}(50)-\mathrm{K}(1)-\mathrm{O}(100)$ & $79.38(15)$ \\
\hline $\mathrm{O}(20)-\mathrm{K}(1)-\mathrm{O}(100)$ & $162.44(15)$ \\
\hline $\mathrm{O}(70)-\mathrm{K}(1)-\mathrm{C}(130)$ & $22.47(16)$ \\
\hline $\mathrm{O}(40)-\mathrm{K}(1)-\mathrm{C}(130)$ & $149.9(2)$ \\
\hline $\mathrm{O}(90)-\mathrm{K}(1)-\mathrm{C}(130)$ & $83.99(18)$ \\
\hline $\mathrm{O}(60)-\mathrm{K}(1)-\mathrm{C}(130)$ & $80.61(17)$ \\
\hline $\mathrm{O}(80)-\mathrm{K}(1)-\mathrm{C}(130)$ & $41.72(17)$ \\
\hline $\mathrm{O}(10)-\mathrm{K}(1)-\mathrm{C}(130)$ & $60.97(18)$ \\
\hline $\mathrm{O}(30)-\mathrm{K}(1)-\mathrm{C}(130)$ & $120.47(17)$ \\
\hline $\mathrm{O}(50)-\mathrm{K}(1)-\mathrm{C}(130)$ & $136.11(17)$ \\
\hline $\mathrm{O}(20)-\mathrm{K}(1)-\mathrm{C}(130)$ & $87.49(17)$ \\
\hline $\mathrm{O}(100)-\mathrm{K}(1)-\mathrm{C}(130)$ & $76.03(17)$ \\
\hline $\mathrm{C}(10)-\mathrm{O}(10)-\mathrm{C}(120)$ & $112.9(7)$ \\
\hline $\mathrm{C}(10)-\mathrm{O}(10)-\mathrm{K}(1)$ & $120.6(5)$ \\
\hline $\mathrm{C}(120)-\mathrm{O}(10)-\mathrm{K}(1)$ & $115.8(5)$ \\
\hline $\mathrm{C}(30)-\mathrm{O}(20)-\mathrm{C}(20)$ & $115.5(7)$ \\
\hline $\mathrm{C}(30)-\mathrm{O}(20)-\mathrm{K}(1)$ & $110.4(5)$ \\
\hline $\mathrm{C}(20)-\mathrm{O}(20)-\mathrm{K}(1)$ & $107.6(5)$ \\
\hline $\mathrm{C}(50)-\mathrm{O}(30)-\mathrm{C}(40)$ & $113.1(6)$ \\
\hline $\mathrm{C}(50)-\mathrm{O}(30)-\mathrm{K}(1)$ & $115.7(5)$ \\
\hline
\end{tabular}




\begin{tabular}{|c|c|}
\hline $\mathrm{C}(40)-\mathrm{O}(30)-\mathrm{K}(1)$ & $119.5(5)$ \\
\hline $\mathrm{C}(70)-\mathrm{O}(40)-\mathrm{C}(60)$ & $113.2(6)$ \\
\hline $\mathrm{C}(70)-\mathrm{O}(40)-\mathrm{K}(1)$ & $117.9(5)$ \\
\hline $\mathrm{C}(60)-\mathrm{O}(40)-\mathrm{K}(1)$ & $113.2(4)$ \\
\hline $\mathrm{C}(90)-\mathrm{O}(50)-\mathrm{C}(80)$ & $113.1(7)$ \\
\hline $\mathrm{C}(90)-\mathrm{O}(50)-\mathrm{K}(1)$ & $112.5(5)$ \\
\hline $\mathrm{C}(80)-\mathrm{O}(50)-\mathrm{K}(1)$ & $111.9(4)$ \\
\hline$C(100)-O(60)-C(110)$ & $115.2(7)$ \\
\hline $\mathrm{C}(100)-\mathrm{O}(60)-\mathrm{K}(1)$ & $116.4(4)$ \\
\hline $\mathrm{C}(110)-\mathrm{O}(60)-\mathrm{K}(1)$ & $112.7(5)$ \\
\hline $\mathrm{C}(200)-\mathrm{O}(70)-\mathrm{C}(130)$ & $113.3(6)$ \\
\hline $\mathrm{C}(200)-\mathrm{O}(70)-\mathrm{K}(1)$ & $128.2(4)$ \\
\hline $\mathrm{C}(130)-\mathrm{O}(70)-\mathrm{K}(1)$ & $108.9(4)$ \\
\hline $\mathrm{C}(140)-\mathrm{O}(80)-\mathrm{C}(150)$ & $113.7(7)$ \\
\hline $\mathrm{C}(140)-\mathrm{O}(80)-\mathrm{K}(1)$ & $117.8(4)$ \\
\hline $\mathrm{C}(150)-\mathrm{O}(80)-\mathrm{K}(1)$ & $110.2(5)$ \\
\hline $\mathrm{C}(160)-\mathrm{O}(90)-\mathrm{C}(170)$ & $116.0(7)$ \\
\hline $\mathrm{C}(160)-\mathrm{O}(90)-\mathrm{K}(1)$ & $120.2(5)$ \\
\hline $\mathrm{C}(170)-\mathrm{O}(90)-\mathrm{K}(1)$ & $111.1(5)$ \\
\hline $\mathrm{C}(190)-\mathrm{O}(100)-\mathrm{C}(180)$ & $113.1(7)$ \\
\hline $\mathrm{C}(190)-\mathrm{O}(100)-\mathrm{K}(1)$ & $108.6(5)$ \\
\hline $\mathrm{C}(180)-\mathrm{O}(100)-\mathrm{K}(1)$ & $116.4(5)$ \\
\hline $\mathrm{O}(10)-\mathrm{C}(10)-\mathrm{C}(20)$ & $108.8(8)$ \\
\hline $\mathrm{O}(10)-\mathrm{C}(10)-\mathrm{H}(10 \mathrm{~A})$ & 109.9 \\
\hline $\mathrm{C}(20)-\mathrm{C}(10)-\mathrm{H}(10 \mathrm{~A})$ & 109.9 \\
\hline $\mathrm{O}(10)-\mathrm{C}(10)-\mathrm{H}(10 \mathrm{~B})$ & 109.9 \\
\hline $\mathrm{C}(20)-\mathrm{C}(10)-\mathrm{H}(10 \mathrm{~B})$ & 109.9 \\
\hline $\mathrm{H}(10 \mathrm{~A})-\mathrm{C}(10)-\mathrm{H}(10 \mathrm{~B})$ & 108.3 \\
\hline $\mathrm{O}(20)-\mathrm{C}(20)-\mathrm{C}(10)$ & $111.7(8)$ \\
\hline $\mathrm{O}(20)-\mathrm{C}(20)-\mathrm{H}(20 \mathrm{~A})$ & 109.3 \\
\hline $\mathrm{C}(10)-\mathrm{C}(20)-\mathrm{H}(20 \mathrm{~A})$ & 109.3 \\
\hline $\mathrm{O}(20)-\mathrm{C}(20)-\mathrm{H}(20 \mathrm{~B})$ & 109.3 \\
\hline $\mathrm{C}(10)-\mathrm{C}(20)-\mathrm{H}(20 \mathrm{~B})$ & 109.3 \\
\hline $\mathrm{H}(20 \mathrm{~A})-\mathrm{C}(20)-\mathrm{H}(20 \mathrm{~B})$ & 107.9 \\
\hline $\mathrm{O}(20)-\mathrm{C}(30)-\mathrm{C}(40)$ & $110.2(7)$ \\
\hline $\mathrm{O}(20)-\mathrm{C}(30)-\mathrm{H}(30 \mathrm{~A})$ & 109.6 \\
\hline
\end{tabular}




\begin{tabular}{|c|c|}
\hline $\mathrm{C}(40)-\mathrm{C}(30)-\mathrm{H}(30 \mathrm{~A})$ & 109.6 \\
\hline $\mathrm{O}(20)-\mathrm{C}(30)-\mathrm{H}(30 \mathrm{~B})$ & 109.6 \\
\hline $\mathrm{C}(40)-\mathrm{C}(30)-\mathrm{H}(30 \mathrm{~B})$ & 109.6 \\
\hline $\mathrm{H}(30 \mathrm{~A})-\mathrm{C}(30)-\mathrm{H}(30 \mathrm{~B})$ & 108.1 \\
\hline $\mathrm{O}(30)-\mathrm{C}(40)-\mathrm{C}(30)$ & $110.5(7)$ \\
\hline $\mathrm{O}(30)-\mathrm{C}(40)-\mathrm{H}(40 \mathrm{C})$ & 109.5 \\
\hline $\mathrm{C}(30)-\mathrm{C}(40)-\mathrm{H}(40 \mathrm{C})$ & 109.5 \\
\hline $\mathrm{O}(30)-\mathrm{C}(40)-\mathrm{H}(40 \mathrm{D})$ & 109.5 \\
\hline $\mathrm{C}(30)-\mathrm{C}(40)-\mathrm{H}(40 \mathrm{D})$ & 109.5 \\
\hline $\mathrm{H}(40 \mathrm{C})-\mathrm{C}(40)-\mathrm{H}(40 \mathrm{D})$ & 108.1 \\
\hline $\mathrm{O}(30)-\mathrm{C}(50)-\mathrm{C}(60)$ & $109.9(7)$ \\
\hline $\mathrm{O}(30)-\mathrm{C}(50)-\mathrm{H}(50 \mathrm{~A})$ & 109.7 \\
\hline $\mathrm{C}(60)-\mathrm{C}(50)-\mathrm{H}(50 \mathrm{~A})$ & 109.7 \\
\hline $\mathrm{O}(30)-\mathrm{C}(50)-\mathrm{H}(50 \mathrm{~B})$ & 109.7 \\
\hline $\mathrm{C}(60)-\mathrm{C}(50)-\mathrm{H}(50 \mathrm{~B})$ & 109.7 \\
\hline $\mathrm{H}(50 \mathrm{~A})-\mathrm{C}(50)-\mathrm{H}(50 \mathrm{~B})$ & 108.2 \\
\hline $\mathrm{O}(40)-\mathrm{C}(60)-\mathrm{C}(50)$ & $112.2(7)$ \\
\hline $\mathrm{O}(40)-\mathrm{C}(60)-\mathrm{H}(60 \mathrm{~A})$ & 109.2 \\
\hline $\mathrm{C}(50)-\mathrm{C}(60)-\mathrm{H}(60 \mathrm{~A})$ & 109.2 \\
\hline $\mathrm{O}(40)-\mathrm{C}(60)-\mathrm{H}(60 \mathrm{~B})$ & 109.2 \\
\hline $\mathrm{C}(50)-\mathrm{C}(60)-\mathrm{H}(60 \mathrm{~B})$ & 109.2 \\
\hline $\mathrm{H}(60 \mathrm{~A})-\mathrm{C}(60)-\mathrm{H}(60 \mathrm{~B})$ & 107.9 \\
\hline $\mathrm{O}(40)-\mathrm{C}(70)-\mathrm{C}(80)$ & $106.1(7)$ \\
\hline $\mathrm{O}(40)-\mathrm{C}(70)-\mathrm{H}(70 \mathrm{~A})$ & 110.5 \\
\hline $\mathrm{C}(80)-\mathrm{C}(70)-\mathrm{H}(70 \mathrm{~A})$ & 110.5 \\
\hline $\mathrm{O}(40)-\mathrm{C}(70)-\mathrm{H}(70 \mathrm{~B})$ & 110.5 \\
\hline $\mathrm{C}(80)-\mathrm{C}(70)-\mathrm{H}(70 \mathrm{~B})$ & 110.5 \\
\hline $\mathrm{H}(70 \mathrm{~A})-\mathrm{C}(70)-\mathrm{H}(70 \mathrm{~B})$ & 108.7 \\
\hline $\mathrm{O}(50)-\mathrm{C}(80)-\mathrm{C}(70)$ & $110.2(6)$ \\
\hline $\mathrm{O}(50)-\mathrm{C}(80)-\mathrm{H}(80 \mathrm{~A})$ & 109.6 \\
\hline $\mathrm{C}(70)-\mathrm{C}(80)-\mathrm{H}(80 \mathrm{~A})$ & 109.6 \\
\hline $\mathrm{O}(50)-\mathrm{C}(80)-\mathrm{H}(80 \mathrm{~B})$ & 109.6 \\
\hline $\mathrm{C}(70)-\mathrm{C}(80)-\mathrm{H}(80 \mathrm{~B})$ & 109.6 \\
\hline $\mathrm{H}(80 \mathrm{~A})-\mathrm{C}(80)-\mathrm{H}(80 \mathrm{~B})$ & 108.1 \\
\hline $\mathrm{O}(50)-\mathrm{C}(90)-\mathrm{C}(100)$ & $110.4(8)$ \\
\hline $\mathrm{O}(50)-\mathrm{C}(90)-\mathrm{H}(90 \mathrm{~A})$ & 109.6 \\
\hline
\end{tabular}




\begin{tabular}{|c|c|}
\hline $\mathrm{C}(100)-\mathrm{C}(90)-\mathrm{H}(90 \mathrm{~A})$ & 109.6 \\
\hline $\mathrm{O}(50)-\mathrm{C}(90)-\mathrm{H}(90 \mathrm{~B})$ & 109.6 \\
\hline $\mathrm{C}(100)-\mathrm{C}(90)-\mathrm{H}(90 \mathrm{~B})$ & 109.6 \\
\hline $\mathrm{H}(90 \mathrm{~A})-\mathrm{C}(90)-\mathrm{H}(90 \mathrm{~B})$ & 108.1 \\
\hline $\mathrm{O}(60)-\mathrm{C}(100)-\mathrm{C}(90)$ & $109.7(7)$ \\
\hline $\mathrm{O}(60)-\mathrm{C}(100)-\mathrm{H}(10 \mathrm{C})$ & 109.7 \\
\hline $\mathrm{C}(90)-\mathrm{C}(100)-\mathrm{H}(10 \mathrm{C})$ & 109.7 \\
\hline $\mathrm{O}(60)-\mathrm{C}(100)-\mathrm{H}(10 \mathrm{D})$ & 109.7 \\
\hline $\mathrm{C}(90)-\mathrm{C}(100)-\mathrm{H}(10 \mathrm{D})$ & 109.7 \\
\hline $\mathrm{H}(10 \mathrm{C})-\mathrm{C}(100)-\mathrm{H}(10 \mathrm{D})$ & 108.2 \\
\hline $\mathrm{O}(60)-\mathrm{C}(110)-\mathrm{C}(120)$ & $112.8(8)$ \\
\hline $\mathrm{O}(60)-\mathrm{C}(110)-\mathrm{H}(11 \mathrm{P})$ & 109.0 \\
\hline $\mathrm{C}(120)-\mathrm{C}(110)-\mathrm{H}(11 \mathrm{P})$ & 109.0 \\
\hline $\mathrm{O}(60)-\mathrm{C}(110)-\mathrm{H}(11 \mathrm{Q})$ & 109.0 \\
\hline $\mathrm{C}(120)-\mathrm{C}(110)-\mathrm{H}(11 \mathrm{Q})$ & 109.0 \\
\hline $\mathrm{H}(11 \mathrm{P})-\mathrm{C}(110)-\mathrm{H}(11 \mathrm{Q})$ & 107.8 \\
\hline $\mathrm{O}(10)-\mathrm{C}(120)-\mathrm{C}(110)$ & $110.8(8)$ \\
\hline $\mathrm{O}(10)-\mathrm{C}(120)-\mathrm{H}(12 \mathrm{M})$ & 109.5 \\
\hline $\mathrm{C}(110)-\mathrm{C}(120)-\mathrm{H}(12 \mathrm{M})$ & 109.5 \\
\hline $\mathrm{O}(10)-\mathrm{C}(120)-\mathrm{H}(12 \mathrm{~N})$ & 109.5 \\
\hline $\mathrm{C}(110)-\mathrm{C}(120)-\mathrm{H}(12 \mathrm{~N})$ & 109.5 \\
\hline $\mathrm{H}(12 \mathrm{M})-\mathrm{C}(120)-\mathrm{H}(12 \mathrm{~N})$ & 108.1 \\
\hline $\mathrm{O}(70)-\mathrm{C}(130)-\mathrm{C}(140)$ & $111.6(7)$ \\
\hline $\mathrm{O}(70)-\mathrm{C}(130)-\mathrm{K}(1)$ & $48.7(3)$ \\
\hline$C(140)-C(130)-K(1)$ & $88.4(4)$ \\
\hline $\mathrm{O}(70)-\mathrm{C}(130)-\mathrm{H}(13 \mathrm{~A})$ & 109.3 \\
\hline $\mathrm{C}(140)-\mathrm{C}(130)-\mathrm{H}(13 \mathrm{~A})$ & 109.3 \\
\hline $\mathrm{K}(1)-\mathrm{C}(130)-\mathrm{H}(13 \mathrm{~A})$ & 78.5 \\
\hline $\mathrm{O}(70)-\mathrm{C}(130)-\mathrm{H}(13 \mathrm{~B})$ & 109.3 \\
\hline $\mathrm{C}(140)-\mathrm{C}(130)-\mathrm{H}(13 \mathrm{~B})$ & 109.3 \\
\hline $\mathrm{K}(1)-\mathrm{C}(130)-\mathrm{H}(13 \mathrm{~B})$ & 156.9 \\
\hline $\mathrm{H}(13 \mathrm{~A})-\mathrm{C}(130)-\mathrm{H}(13 \mathrm{~B})$ & 108.0 \\
\hline $\mathrm{O}(80)-\mathrm{C}(140)-\mathrm{C}(130)$ & $108.1(6)$ \\
\hline $\mathrm{O}(80)-\mathrm{C}(140)-\mathrm{H}(14 \mathrm{D})$ & 110.1 \\
\hline $\mathrm{C}(130)-\mathrm{C}(140)-\mathrm{H}(14 \mathrm{D})$ & 110.1 \\
\hline $\mathrm{O}(80)-\mathrm{C}(140)-\mathrm{H}(14 \mathrm{E})$ & 110.1 \\
\hline
\end{tabular}




\begin{tabular}{|c|c|}
\hline $\mathrm{C}(130)-\mathrm{C}(140)-\mathrm{H}(14 \mathrm{E})$ & 110.1 \\
\hline $\mathrm{H}(14 \mathrm{D})-\mathrm{C}(140)-\mathrm{H}(14 \mathrm{E})$ & 108.4 \\
\hline $\mathrm{O}(80)-\mathrm{C}(150)-\mathrm{C}(160)$ & $113.4(7)$ \\
\hline $\mathrm{O}(80)-\mathrm{C}(150)-\mathrm{H}(15 \mathrm{~A})$ & 108.9 \\
\hline $\mathrm{C}(160)-\mathrm{C}(150)-\mathrm{H}(15 \mathrm{~A})$ & 108.9 \\
\hline $\mathrm{O}(80)-\mathrm{C}(150)-\mathrm{H}(15 \mathrm{~B})$ & 108.9 \\
\hline $\mathrm{C}(160)-\mathrm{C}(150)-\mathrm{H}(15 \mathrm{~B})$ & 108.9 \\
\hline $\mathrm{H}(15 \mathrm{~A})-\mathrm{C}(150)-\mathrm{H}(15 \mathrm{~B})$ & 107.7 \\
\hline $\mathrm{O}(90)-\mathrm{C}(160)-\mathrm{C}(150)$ & $108.6(7)$ \\
\hline $\mathrm{O}(90)-\mathrm{C}(160)-\mathrm{H}(16 \mathrm{~A})$ & 110.0 \\
\hline$C(150)-C(160)-H(16 A)$ & 110.0 \\
\hline $\mathrm{O}(90)-\mathrm{C}(160)-\mathrm{H}(16 \mathrm{~B})$ & 110.0 \\
\hline $\mathrm{C}(150)-\mathrm{C}(160)-\mathrm{H}(16 \mathrm{~B})$ & 110.0 \\
\hline $\mathrm{H}(16 \mathrm{~A})-\mathrm{C}(160)-\mathrm{H}(16 \mathrm{~B})$ & 108.4 \\
\hline $\mathrm{O}(90)-\mathrm{C}(170)-\mathrm{C}(180)$ & $112.9(7)$ \\
\hline $\mathrm{O}(90)-\mathrm{C}(170)-\mathrm{H}(17 \mathrm{~A})$ & 109.0 \\
\hline $\mathrm{C}(180)-\mathrm{C}(170)-\mathrm{H}(17 \mathrm{~A})$ & 109.0 \\
\hline $\mathrm{O}(90)-\mathrm{C}(170)-\mathrm{H}(17 \mathrm{~B})$ & 109.0 \\
\hline $\mathrm{C}(180)-\mathrm{C}(170)-\mathrm{H}(17 \mathrm{~B})$ & 109.0 \\
\hline $\mathrm{H}(17 \mathrm{~A})-\mathrm{C}(170)-\mathrm{H}(17 \mathrm{~B})$ & 107.8 \\
\hline $\mathrm{O}(100)-\mathrm{C}(180)-\mathrm{C}(170)$ & $107.8(8)$ \\
\hline $\mathrm{O}(100)-\mathrm{C}(180)-\mathrm{H}(18 \mathrm{~A})$ & 110.2 \\
\hline $\mathrm{C}(170)-\mathrm{C}(180)-\mathrm{H}(18 \mathrm{~A})$ & 110.2 \\
\hline $\mathrm{O}(100)-\mathrm{C}(180)-\mathrm{H}(18 \mathrm{~B})$ & 110.2 \\
\hline $\mathrm{C}(170)-\mathrm{C}(180)-\mathrm{H}(18 \mathrm{~B})$ & 110.2 \\
\hline $\mathrm{H}(18 \mathrm{~A})-\mathrm{C}(180)-\mathrm{H}(18 \mathrm{~B})$ & 108.5 \\
\hline $\mathrm{O}(100)-\mathrm{C}(190)-\mathrm{C}(200)$ & $113.3(7)$ \\
\hline O(100)-C(190)-H(19A) & 108.9 \\
\hline $\mathrm{C}(200)-\mathrm{C}(190)-\mathrm{H}(19 \mathrm{~A})$ & 108.9 \\
\hline $\mathrm{O}(100)-\mathrm{C}(190)-\mathrm{H}(19 \mathrm{~B})$ & 108.9 \\
\hline $\mathrm{C}(200)-\mathrm{C}(190)-\mathrm{H}(19 \mathrm{~B})$ & 108.9 \\
\hline H(19A)-C(190)-H(19B) & 107.7 \\
\hline $\mathrm{O}(70)-\mathrm{C}(200)-\mathrm{C}(190)$ & $107.2(6)$ \\
\hline $\mathrm{O}(70)-\mathrm{C}(200)-\mathrm{H}(20 \mathrm{C})$ & 110.3 \\
\hline $\mathrm{C}(190)-\mathrm{C}(200)-\mathrm{H}(20 \mathrm{C})$ & 110.3 \\
\hline $\mathrm{O}(70)-\mathrm{C}(200)-\mathrm{H}(20 \mathrm{D})$ & 110.3 \\
\hline
\end{tabular}




\begin{tabular}{|c|c|}
\hline $\mathrm{C}(190)-\mathrm{C}(200)-\mathrm{H}(20 \mathrm{D})$ & 110.3 \\
\hline $\mathrm{H}(20 \mathrm{C})-\mathrm{C}(200)-\mathrm{H}(20 \mathrm{D})$ & 108.5 \\
\hline $\mathrm{O}(160)-\mathrm{K}(2)-\mathrm{O}(160) \# 1$ & $83.1(2)$ \\
\hline $\mathrm{O}(160)-\mathrm{K}(2)-\mathrm{O}(120) \# 1$ & $136.91(16)$ \\
\hline $\mathrm{O}(160) \# 1-\mathrm{K}(2)-\mathrm{O}(120) \# 1$ & $68.06(14)$ \\
\hline $\mathrm{O}(160)-\mathrm{K}(2)-\mathrm{O}(120)$ & $68.07(14)$ \\
\hline $\mathrm{O}(160) \# 1-\mathrm{K}(2)-\mathrm{O}(120)$ & $136.91(16)$ \\
\hline $\mathrm{O}(120) \# 1-\mathrm{K}(2)-\mathrm{O}(120)$ & $112.6(2)$ \\
\hline $\mathrm{O}(160)-\mathrm{K}(2)-\mathrm{O}(150)$ & $57.82(13)$ \\
\hline $\mathrm{O}(160) \# 1-\mathrm{K}(2)-\mathrm{O}(150)$ & $57.82(13)$ \\
\hline $\mathrm{O}(120) \# 1-\mathrm{K}(2)-\mathrm{O}(150)$ & $79.50(13)$ \\
\hline $\mathrm{O}(120)-\mathrm{K}(2)-\mathrm{O}(150)$ & $79.50(13)$ \\
\hline $\mathrm{O}(160)-\mathrm{K}(2)-\mathrm{O}(140)$ & $113.56(14)$ \\
\hline $\mathrm{O}(160) \# 1-\mathrm{K}(2)-\mathrm{O}(140)$ & $113.56(13)$ \\
\hline $\mathrm{O}(120) \# 1-\mathrm{K}(2)-\mathrm{O}(140)$ & $107.24(13)$ \\
\hline $\mathrm{O}(120)-\mathrm{K}(2)-\mathrm{O}(140)$ & $107.24(13)$ \\
\hline $\mathrm{O}(150)-\mathrm{K}(2)-\mathrm{O}(140)$ & $166.90(19)$ \\
\hline $\mathrm{O}(160)-\mathrm{K}(2)-\mathrm{O}(110)$ & $117.38(14)$ \\
\hline $\mathrm{O}(160) \# 1-\mathrm{K}(2)-\mathrm{O}(110)$ & $117.38(14)$ \\
\hline $\mathrm{O}(120) \# 1-\mathrm{K}(2)-\mathrm{O}(110)$ & $57.19(12)$ \\
\hline $\mathrm{O}(120)-\mathrm{K}(2)-\mathrm{O}(110)$ & $57.19(12)$ \\
\hline $\mathrm{O}(150)-\mathrm{K}(2)-\mathrm{O}(110)$ & $83.32(19)$ \\
\hline $\mathrm{O}(140)-\mathrm{K}(2)-\mathrm{O}(110)$ & $109.78(19)$ \\
\hline $\mathrm{O}(160)-\mathrm{K}(2)-\mathrm{O}(130) \# 1$ & $142.95(15)$ \\
\hline $\mathrm{O}(160) \# 1-\mathrm{K}(2)-\mathrm{O}(130) \# 1$ & $72.48(14)$ \\
\hline $\mathrm{O}(120) \# 1-\mathrm{K}(2)-\mathrm{O}(130) \# 1$ & $57.18(14)$ \\
\hline $\mathrm{O}(120)-\mathrm{K}(2)-\mathrm{O}(130) \# 1$ & $146.71(16)$ \\
\hline $\mathrm{O}(150)-\mathrm{K}(2)-\mathrm{O}(130) \# 1$ & $123.60(10)$ \\
\hline $\mathrm{O}(140)-\mathrm{K}(2)-\mathrm{O}(130) \# 1$ & $56.02(10)$ \\
\hline $\mathrm{O}(110)-\mathrm{K}(2)-\mathrm{O}(130) \# 1$ & $98.72(13)$ \\
\hline $\mathrm{O}(160)-\mathrm{K}(2)-\mathrm{O}(130)$ & $72.48(14)$ \\
\hline $\mathrm{O}(160) \# 1-\mathrm{K}(2)-\mathrm{O}(130)$ & $142.95(15)$ \\
\hline $\mathrm{O}(120) \# 1-\mathrm{K}(2)-\mathrm{O}(130)$ & $146.71(16)$ \\
\hline $\mathrm{O}(120)-\mathrm{K}(2)-\mathrm{O}(130)$ & $57.18(14)$ \\
\hline $\mathrm{O}(150)-\mathrm{K}(2)-\mathrm{O}(130)$ & $123.60(10)$ \\
\hline $\mathrm{O}(140)-\mathrm{K}(2)-\mathrm{O}(130)$ & $56.02(10)$ \\
\hline
\end{tabular}




\begin{tabular}{|c|c|}
\hline $\mathrm{O}(110)-\mathrm{K}(2)-\mathrm{O}(130)$ & $98.72(13)$ \\
\hline $\mathrm{O}(130) \# 1-\mathrm{K}(2)-\mathrm{O}(130)$ & $111.8(2)$ \\
\hline $\mathrm{O}(160)-\mathrm{K}(2)-\mathrm{O}(170)$ & $57.36(13)$ \\
\hline $\mathrm{O}(160) \# 1-\mathrm{K}(2)-\mathrm{O}(170)$ & $57.36(13)$ \\
\hline $\mathrm{O}(120) \# 1-\mathrm{K}(2)-\mathrm{O}(170)$ & $121.28(12)$ \\
\hline $\mathrm{O}(120)-\mathrm{K}(2)-\mathrm{O}(170)$ & $121.28(12)$ \\
\hline $\mathrm{O}(150)-\mathrm{K}(2)-\mathrm{O}(170)$ & $88.48(19)$ \\
\hline $\mathrm{O}(140)-\mathrm{K}(2)-\mathrm{O}(170)$ & $78.41(18)$ \\
\hline $\mathrm{O}(110)-\mathrm{K}(2)-\mathrm{O}(170)$ & $171.8(2)$ \\
\hline $\mathrm{O}(130) \# 1-\mathrm{K}(2)-\mathrm{O}(170)$ & $85.80(13)$ \\
\hline $\mathrm{O}(130)-\mathrm{K}(2)-\mathrm{O}(170)$ & $85.80(13)$ \\
\hline $\mathrm{C}(210)-\mathrm{O}(110)-\mathrm{C}(210) \# 1$ & $112.6(9)$ \\
\hline $\mathrm{C}(210)-\mathrm{O}(110)-\mathrm{K}(2)$ & $114.5(5)$ \\
\hline $\mathrm{C}(210) \# 1-\mathrm{O}(110)-\mathrm{K}(2)$ & $114.5(5)$ \\
\hline $\mathrm{C}(220)-\mathrm{O}(120)-\mathrm{C}(230)$ & $114.3(6)$ \\
\hline $\mathrm{C}(220)-\mathrm{O}(120)-\mathrm{K}(2)$ & $116.8(5)$ \\
\hline $\mathrm{C}(230)-\mathrm{O}(120)-\mathrm{K}(2)$ & $114.3(4)$ \\
\hline $\mathrm{C}(250)-\mathrm{O}(130)-\mathrm{C}(240)$ & $111.7(6)$ \\
\hline $\mathrm{C}(250)-\mathrm{O}(130)-\mathrm{K}(2)$ & $118.8(4)$ \\
\hline $\mathrm{C}(240)-\mathrm{O}(130)-\mathrm{K}(2)$ & $118.0(4)$ \\
\hline $\mathrm{C}(260)-\mathrm{O}(140)-\mathrm{C}(260) \# 1$ & $110.7(7)$ \\
\hline $\mathrm{C}(260)-\mathrm{O}(140)-\mathrm{K}(2)$ & $111.1(4)$ \\
\hline $\mathrm{C}(260) \# 1-\mathrm{O}(140)-\mathrm{K}(2)$ & $111.1(4)$ \\
\hline $\mathrm{C}(8 \mathrm{Y}) \# 1-\mathrm{O}(150)-\mathrm{C}(8 \mathrm{Y})$ & $135(3)$ \\
\hline $\mathrm{C}(8 \mathrm{X}) \# 1-\mathrm{O}(150)-\mathrm{C}(8 \mathrm{X})$ & $108.5(17)$ \\
\hline $\mathrm{C}(8 \mathrm{Y}) \# 1-\mathrm{O}(150)-\mathrm{K}(2)$ & $102.9(10)$ \\
\hline $\mathrm{C}(8 \mathrm{Y})-\mathrm{O}(150)-\mathrm{K}(2)$ & $102.9(10)$ \\
\hline $\mathrm{C}(8 \mathrm{X}) \# 1-\mathrm{O}(150)-\mathrm{K}(2)$ & $120.0(7)$ \\
\hline $\mathrm{C}(8 \mathrm{X})-\mathrm{O}(150)-\mathrm{K}(2)$ & $120.0(8)$ \\
\hline $\mathrm{C}(6 \mathrm{X})-\mathrm{O}(160)-\mathrm{C}(7 \mathrm{X})$ & $108.7(9)$ \\
\hline $\mathrm{C}(7 \mathrm{Y})-\mathrm{O}(160)-\mathrm{C}(6 \mathrm{Y})$ & $110.4(10)$ \\
\hline $\mathrm{C}(7 \mathrm{Y})-\mathrm{O}(160)-\mathrm{K}(2)$ & $129.1(7)$ \\
\hline $\mathrm{C}(6 \mathrm{X})-\mathrm{O}(160)-\mathrm{K}(2)$ & $128.5(7)$ \\
\hline $\mathrm{C}(7 \mathrm{X})-\mathrm{O}(160)-\mathrm{K}(2)$ & $107.8(7)$ \\
\hline $\mathrm{C}(6 \mathrm{Y})-\mathrm{O}(160)-\mathrm{K}(2)$ & $109.5(7)$ \\
\hline $\mathrm{O}(110)-\mathrm{C}(210)-\mathrm{C}(220)$ & $109.6(7)$ \\
\hline
\end{tabular}




\begin{tabular}{|c|c|}
\hline $\mathrm{O}(110)-\mathrm{C}(210)-\mathrm{H}(21 \mathrm{~A})$ & 109.7 \\
\hline $\mathrm{C}(220)-\mathrm{C}(210)-\mathrm{H}(21 \mathrm{~A})$ & 109.7 \\
\hline $\mathrm{O}(110)-\mathrm{C}(210)-\mathrm{H}(21 \mathrm{~B})$ & 109.7 \\
\hline $\mathrm{C}(220)-\mathrm{C}(210)-\mathrm{H}(21 \mathrm{~B})$ & 109.7 \\
\hline $\mathrm{H}(21 \mathrm{~A})-\mathrm{C}(210)-\mathrm{H}(21 \mathrm{~B})$ & 108.2 \\
\hline $\mathrm{O}(120)-\mathrm{C}(220)-\mathrm{C}(210)$ & $110.2(7)$ \\
\hline $\mathrm{O}(120)-\mathrm{C}(220)-\mathrm{H}(22 \mathrm{~A})$ & 109.6 \\
\hline $\mathrm{C}(210)-\mathrm{C}(220)-\mathrm{H}(22 \mathrm{~A})$ & 109.6 \\
\hline $\mathrm{O}(120)-\mathrm{C}(220)-\mathrm{H}(22 \mathrm{~B})$ & 109.6 \\
\hline $\mathrm{C}(210)-\mathrm{C}(220)-\mathrm{H}(22 \mathrm{~B})$ & 109.6 \\
\hline $\mathrm{H}(22 \mathrm{~A})-\mathrm{C}(220)-\mathrm{H}(22 \mathrm{~B})$ & 108.1 \\
\hline $\mathrm{O}(120)-\mathrm{C}(230)-\mathrm{C}(240)$ & $111.3(6)$ \\
\hline $\mathrm{O}(120)-\mathrm{C}(230)-\mathrm{H}(23 \mathrm{~A})$ & 109.4 \\
\hline $\mathrm{C}(240)-\mathrm{C}(230)-\mathrm{H}(23 \mathrm{~A})$ & 109.4 \\
\hline $\mathrm{O}(120)-\mathrm{C}(230)-\mathrm{H}(23 \mathrm{~B})$ & 109.4 \\
\hline $\mathrm{C}(240)-\mathrm{C}(230)-\mathrm{H}(23 \mathrm{~B})$ & 109.4 \\
\hline $\mathrm{H}(23 \mathrm{~A})-\mathrm{C}(230)-\mathrm{H}(23 \mathrm{~B})$ & 108.0 \\
\hline $\mathrm{O}(130)-\mathrm{C}(240)-\mathrm{C}(230)$ & $108.7(6)$ \\
\hline $\mathrm{O}(130)-\mathrm{C}(240)-\mathrm{H}(24 \mathrm{~A})$ & 110.0 \\
\hline $\mathrm{C}(230)-\mathrm{C}(240)-\mathrm{H}(24 \mathrm{~A})$ & 110.0 \\
\hline $\mathrm{O}(130)-\mathrm{C}(240)-\mathrm{H}(24 \mathrm{~B})$ & 110.0 \\
\hline $\mathrm{C}(230)-\mathrm{C}(240)-\mathrm{H}(24 \mathrm{~B})$ & 110.0 \\
\hline $\mathrm{H}(24 \mathrm{~A})-\mathrm{C}(240)-\mathrm{H}(24 \mathrm{~B})$ & 108.3 \\
\hline $\mathrm{O}(130)-\mathrm{C}(250)-\mathrm{C}(260)$ & $109.9(6)$ \\
\hline $\mathrm{O}(130)-\mathrm{C}(250)-\mathrm{H}(25 \mathrm{~A})$ & 109.7 \\
\hline $\mathrm{C}(260)-\mathrm{C}(250)-\mathrm{H}(25 \mathrm{~A})$ & 109.7 \\
\hline $\mathrm{O}(130)-\mathrm{C}(250)-\mathrm{H}(25 \mathrm{~B})$ & 109.7 \\
\hline $\mathrm{C}(260)-\mathrm{C}(250)-\mathrm{H}(25 \mathrm{~B})$ & 109.7 \\
\hline $\mathrm{H}(25 \mathrm{~A})-\mathrm{C}(250)-\mathrm{H}(25 \mathrm{~B})$ & 108.2 \\
\hline $\mathrm{O}(140)-\mathrm{C}(260)-\mathrm{C}(250)$ & $107.4(6)$ \\
\hline $\mathrm{O}(140)-\mathrm{C}(260)-\mathrm{H}(26 \mathrm{~A})$ & 110.2 \\
\hline $\mathrm{C}(250)-\mathrm{C}(260)-\mathrm{H}(26 \mathrm{~A})$ & 110.2 \\
\hline $\mathrm{O}(140)-\mathrm{C}(260)-\mathrm{H}(26 \mathrm{~B})$ & 110.2 \\
\hline $\mathrm{C}(250)-\mathrm{C}(260)-\mathrm{H}(26 \mathrm{~B})$ & 110.2 \\
\hline $\mathrm{H}(26 \mathrm{~A})-\mathrm{C}(260)-\mathrm{H}(26 \mathrm{~B})$ & 108.5 \\
\hline $\mathrm{C}(7 \mathrm{X})-\mathrm{C}(8 \mathrm{X})-\mathrm{O}(150)$ & $106.9(15)$ \\
\hline
\end{tabular}




\begin{tabular}{|c|c|}
\hline $\mathrm{C}(7 \mathrm{X})-\mathrm{C}(8 \mathrm{X})-\mathrm{H}(8 \mathrm{XA})$ & 110.3 \\
\hline $\mathrm{O}(150)-\mathrm{C}(8 \mathrm{X})-\mathrm{H}(8 \mathrm{XA})$ & 110.3 \\
\hline $\mathrm{C}(7 \mathrm{X})-\mathrm{C}(8 \mathrm{X})-\mathrm{H}(8 \mathrm{XB})$ & 110.3 \\
\hline $\mathrm{O}(150)-\mathrm{C}(8 \mathrm{X})-\mathrm{H}(8 \mathrm{XB})$ & 110.3 \\
\hline $\mathrm{H}(8 \mathrm{XA})-\mathrm{C}(8 \mathrm{X})-\mathrm{H}(8 \mathrm{XB})$ & 108.6 \\
\hline $\mathrm{C}(8 \mathrm{Y})-\mathrm{C}(7 \mathrm{Y})-\mathrm{O}(160)$ & $99.1(14)$ \\
\hline $\mathrm{C}(8 \mathrm{Y})-\mathrm{C}(7 \mathrm{Y})-\mathrm{H}(7 \mathrm{YA})$ & 111.9 \\
\hline $\mathrm{O}(160)-\mathrm{C}(7 \mathrm{Y})-\mathrm{H}(7 \mathrm{YA})$ & 111.9 \\
\hline $\mathrm{C}(8 \mathrm{Y})-\mathrm{C}(7 \mathrm{Y})-\mathrm{H}(7 \mathrm{YB})$ & 111.9 \\
\hline $\mathrm{O}(160)-\mathrm{C}(7 \mathrm{Y})-\mathrm{H}(7 \mathrm{YB})$ & 111.9 \\
\hline H(7YA)-C(7Y)-H(7YB) & 109.6 \\
\hline $\mathrm{C}(5 \mathrm{X})-\mathrm{C}(6 \mathrm{X})-\mathrm{O}(160)$ & $97.4(13)$ \\
\hline $\mathrm{C}(5 \mathrm{X})-\mathrm{C}(6 \mathrm{X})-\mathrm{H}(6 \mathrm{XA})$ & 112.3 \\
\hline $\mathrm{O}(160)-\mathrm{C}(6 \mathrm{X})-\mathrm{H}(6 \mathrm{XA})$ & 112.3 \\
\hline $\mathrm{C}(5 \mathrm{X})-\mathrm{C}(6 \mathrm{X})-\mathrm{H}(6 \mathrm{XB})$ & 112.3 \\
\hline $\mathrm{O}(160)-\mathrm{C}(6 \mathrm{X})-\mathrm{H}(6 \mathrm{XB})$ & 112.3 \\
\hline $\mathrm{H}(6 \mathrm{XA})-\mathrm{C}(6 \mathrm{X})-\mathrm{H}(6 \mathrm{XB})$ & 109.9 \\
\hline $\mathrm{C}(6 \mathrm{Y})-\mathrm{C}(5 \mathrm{Y})-\mathrm{O}(170)$ & $108.6(14)$ \\
\hline $\mathrm{C}(6 \mathrm{Y})-\mathrm{C}(5 \mathrm{Y})-\mathrm{H}(5 \mathrm{YA})$ & 110.0 \\
\hline $\mathrm{O}(170)-\mathrm{C}(5 \mathrm{Y})-\mathrm{H}(5 \mathrm{YA})$ & 110.0 \\
\hline $\mathrm{C}(6 \mathrm{Y})-\mathrm{C}(5 \mathrm{Y})-\mathrm{H}(5 \mathrm{YB})$ & 110.0 \\
\hline $\mathrm{O}(170)-\mathrm{C}(5 \mathrm{Y})-\mathrm{H}(5 \mathrm{YB})$ & 110.0 \\
\hline $\mathrm{H}(5 \mathrm{YA})-\mathrm{C}(5 \mathrm{Y})-\mathrm{H}(5 \mathrm{YB})$ & 108.3 \\
\hline $\mathrm{C}(5 \mathrm{Y}) \# 1-\mathrm{O}(170)-\mathrm{C}(5 \mathrm{Y})$ & $104.5(15)$ \\
\hline $\mathrm{C}(5 \mathrm{X})-\mathrm{O}(170)-\mathrm{C}(5 \mathrm{X}) \# 1$ & $134(2)$ \\
\hline $\mathrm{C}(5 \mathrm{Y}) \# 1-\mathrm{O}(170)-\mathrm{K}(2)$ & $119.5(7)$ \\
\hline $\mathrm{C}(5 \mathrm{Y})-\mathrm{O}(170)-\mathrm{K}(2)$ & $119.5(7)$ \\
\hline $\mathrm{C}(5 \mathrm{X})-\mathrm{O}(170)-\mathrm{K}(2)$ & $102.3(10)$ \\
\hline $\mathrm{C}(5 \mathrm{X}) \# 1-\mathrm{O}(170)-\mathrm{K}(2)$ & $102.3(10)$ \\
\hline $\mathrm{O}(230) \# 1-\mathrm{K}(3)-\mathrm{O}(230)$ & $58.3(2)$ \\
\hline $\mathrm{O}(230) \# 1-\mathrm{K}(3)-\mathrm{O}(190)$ & $158.2(3)$ \\
\hline $\mathrm{O}(230)-\mathrm{K}(3)-\mathrm{O}(190)$ & $100.4(3)$ \\
\hline $\mathrm{O}(230) \# 1-\mathrm{K}(3)-\mathrm{O}(190) \# 1$ & $100.4(3)$ \\
\hline $\mathrm{O}(230)-\mathrm{K}(3)-\mathrm{O}(190) \# 1$ & $158.2(3)$ \\
\hline $\mathrm{O}(190)-\mathrm{K}(3)-\mathrm{O}(190) \# 1$ & $100.2(4)$ \\
\hline $\mathrm{O}(230) \# 1-\mathrm{K}(3)-\mathrm{O}(180)$ & $129.46(18)$ \\
\hline
\end{tabular}




\begin{tabular}{|c|c|}
\hline $\mathrm{O}(230)-\mathrm{K}(3)-\mathrm{O}(180)$ & $129.46(19)$ \\
\hline $\mathrm{O}(190)-\mathrm{K}(3)-\mathrm{O}(180)$ & $58.50(17)$ \\
\hline $\mathrm{O}(190) \# 1-\mathrm{K}(3)-\mathrm{O}(180)$ & $58.50(17)$ \\
\hline $\mathrm{O}(230) \# 1-\mathrm{K}(3)-\mathrm{O}(200) \# 1$ & $69.4(4)$ \\
\hline $\mathrm{O}(230)-\mathrm{K}(3)-\mathrm{O}(200) \# 1$ & $114.4(3)$ \\
\hline $\mathrm{O}(190)-\mathrm{K}(3)-\mathrm{O}(200) \# 1$ & $129.4(5)$ \\
\hline $\mathrm{O}(190) \# 1-\mathrm{K}(3)-\mathrm{O}(200) \# 1$ & $55.2(2)$ \\
\hline $\mathrm{O}(180)-\mathrm{K}(3)-\mathrm{O}(200) \# 1$ & $113.4(2)$ \\
\hline $\mathrm{O}(230) \# 1-\mathrm{K}(3)-\mathrm{O}(200)$ & $114.4(3)$ \\
\hline $\mathrm{O}(230)-\mathrm{K}(3)-\mathrm{O}(200)$ & $69.4(4)$ \\
\hline $\mathrm{O}(190)-\mathrm{K}(3)-\mathrm{O}(200)$ & $55.2(2)$ \\
\hline $\mathrm{O}(190) \# 1-\mathrm{K}(3)-\mathrm{O}(200)$ & $129.4(5)$ \\
\hline $\mathrm{O}(180)-\mathrm{K}(3)-\mathrm{O}(200)$ & $113.4(2)$ \\
\hline $\mathrm{O}(200) \# 1-\mathrm{K}(3)-\mathrm{O}(200)$ & $103.5(7)$ \\
\hline $\mathrm{O}(230) \# 1-\mathrm{K}(3)-\mathrm{O}(220)$ & $86.15(18)$ \\
\hline $\mathrm{O}(230)-\mathrm{K}(3)-\mathrm{O}(220)$ & $57.74(16)$ \\
\hline $\mathrm{O}(190)-\mathrm{K}(3)-\mathrm{O}(220)$ & $77.3(2)$ \\
\hline $\mathrm{O}(190) \# 1-\mathrm{K}(3)-\mathrm{O}(220)$ & $121.0(2)$ \\
\hline $\mathrm{O}(180)-\mathrm{K}(3)-\mathrm{O}(220)$ & $72.3(2)$ \\
\hline $\mathrm{O}(200) \# 1-\mathrm{K}(3)-\mathrm{O}(220)$ & $152.6(4)$ \\
\hline $\mathrm{O}(200)-\mathrm{K}(3)-\mathrm{O}(220)$ & $97.7(4)$ \\
\hline $\mathrm{O}(230) \# 1-\mathrm{K}(3)-\mathrm{O}(220) \# 1$ & $57.74(16)$ \\
\hline $\mathrm{O}(230)-\mathrm{K}(3)-\mathrm{O}(220) \# 1$ & $86.15(18)$ \\
\hline $\mathrm{O}(190)-\mathrm{K}(3)-\mathrm{O}(220) \# 1$ & $121.0(2)$ \\
\hline $\mathrm{O}(190) \# 1-\mathrm{K}(3)-\mathrm{O}(220) \# 1$ & $77.3(2)$ \\
\hline $\mathrm{O}(180)-\mathrm{K}(3)-\mathrm{O}(220) \# 1$ & $72.3(2)$ \\
\hline $\mathrm{O}(200) \# 1-\mathrm{K}(3)-\mathrm{O}(220) \# 1$ & $97.7(4)$ \\
\hline $\mathrm{O}(200)-\mathrm{K}(3)-\mathrm{O}(220) \# 1$ & $152.6(4)$ \\
\hline $\mathrm{O}(220)-\mathrm{K}(3)-\mathrm{O}(220) \# 1$ & $57.3(2)$ \\
\hline $\mathrm{O}(230) \# 1-\mathrm{K}(3)-\mathrm{O}(210)$ & $74.8(3)$ \\
\hline $\mathrm{O}(230)-\mathrm{K}(3)-\mathrm{O}(210)$ & $74.8(3)$ \\
\hline $\mathrm{O}(190)-\mathrm{K}(3)-\mathrm{O}(210)$ & $105.9(2)$ \\
\hline $\mathrm{O}(190) \# 1-\mathrm{K}(3)-\mathrm{O}(210)$ & $105.9(2)$ \\
\hline $\mathrm{O}(180)-\mathrm{K}(3)-\mathrm{O}(210)$ & $150.8(4)$ \\
\hline $\mathrm{O}(200) \# 1-\mathrm{K}(3)-\mathrm{O}(210)$ & $54.7(3)$ \\
\hline $\mathrm{O}(200)-\mathrm{K}(3)-\mathrm{O}(210)$ & $54.7(3)$ \\
\hline
\end{tabular}




$\begin{array}{ll}\mathrm{O}(220)-\mathrm{K}(3)-\mathrm{O}(210) & 131.8(3) \\ \mathrm{O}(220) \# 1-\mathrm{K}(3)-\mathrm{O}(210) & 131.8(3) \\ \mathrm{C}(310) \# 1-\mathrm{O}(180)-\mathrm{C}(310) & 134.0(15) \\ \mathrm{C}(310) \# 1-\mathrm{O}(180)-\mathrm{K}(3) & 111.3(7) \\ \mathrm{C}(310)-\mathrm{O}(180)-\mathrm{K}(3) & 111.3(7) \\ \mathrm{C}(330)-\mathrm{O}(190)-\mathrm{C}(320) & 116.4(9) \\ \mathrm{C}(330)-\mathrm{O}(190)-\mathrm{K}(3) & 123.0(7) \\ \mathrm{C}(320)-\mathrm{O}(190)-\mathrm{K}(3) & 120.5(7) \\ \mathrm{C}(340)-\mathrm{O}(200)-\mathrm{C}(350) & 123.7(11) \\ \mathrm{C}(340)-\mathrm{O}(200)-\mathrm{K}(3) & 120.2(8) \\ \mathrm{C}(350)-\mathrm{O}(200)-\mathrm{K}(3) & 115.8(8) \\ \mathrm{C}(370)-\mathrm{O}(220)-\mathrm{C}(380) & 115.1(11) \\ \mathrm{C}(370)-\mathrm{O}(220)-\mathrm{K}(3) & 115.3(6) \\ \mathrm{C}(380)-\mathrm{O}(220)-\mathrm{K}(3) & 115.0(6) \\ \mathrm{C}(400)-\mathrm{O}(230)-\mathrm{C}(390) & 116.2(9) \\ \mathrm{C}(400)-\mathrm{O}(230)-\mathrm{K}(3) & 115.1(6) \\ \mathrm{C}(390)-\mathrm{O}(230)-\mathrm{K}(3) & 116.8(6) \\ \mathrm{C}(320)-\mathrm{C}(310)-\mathrm{O}(180) & 127.5(11) \\ \mathrm{C}(320)-\mathrm{C}(310)-\mathrm{H}(31 \mathrm{~A}) & 105.4 \\ \mathrm{O}(180)-\mathrm{C}(310)-\mathrm{H}(31 \mathrm{~A}) & 105.4 \\ \mathrm{C}(320)-\mathrm{C}(310)-\mathrm{H}(31 \mathrm{~B}) & 105.4 \\ \mathrm{O}(180)-\mathrm{C}(310)-\mathrm{H}(31 \mathrm{~B}) & 105.4 \\ \mathrm{H}(31 \mathrm{~A})-\mathrm{C}(310)-\mathrm{H}(31 \mathrm{~B}) & 106.0 \\ \mathrm{C}(310)-\mathrm{C}(320)-\mathrm{O}(190) & 115.1(11) \\ \mathrm{C}(310)-\mathrm{C}(320)-\mathrm{H}(32 \mathrm{~A}) & 108.5 \\ \mathrm{O}(190)-\mathrm{C}(320)-\mathrm{H}(32 \mathrm{~A}) & 108.5 \\ \mathrm{C}(310)-\mathrm{C}(320)-\mathrm{H}(32 \mathrm{~B}) & 108.5 \\ \mathrm{O}(190)-\mathrm{C}(320)-\mathrm{H}(32 \mathrm{~B}) & 108.5 \\ \mathrm{H}(32 \mathrm{~A})-\mathrm{C}(320)-\mathrm{H}(32 \mathrm{~B}) & 107.5 \\ \mathrm{O}(190)-\mathrm{C}(330)-\mathrm{C}(340) & 116.2(10) \\ \mathrm{O}(190)-\mathrm{C}(330)-\mathrm{H}(33 \mathrm{~A}) & 108.2 \\ \mathrm{C}(340)-\mathrm{C}(330)-\mathrm{H}(33 \mathrm{~A}) & 108.2 \\ & 108.2 \\ \mathrm{O}(30)-\mathrm{C}(330)-\mathrm{H}(33 \mathrm{~B}) & 108.2 \\ \mathrm{C}(330)-\mathrm{C}(350) & 110.5(15) \\ & \end{array}$




\begin{tabular}{|c|c|}
\hline $\mathrm{O}(210)-\mathrm{C}(360)-\mathrm{H}(36 \mathrm{~A})$ & 109.5 \\
\hline $\mathrm{C}(350)-\mathrm{C}(360)-\mathrm{H}(36 \mathrm{~A})$ & 109.5 \\
\hline $\mathrm{O}(210)-\mathrm{C}(360)-\mathrm{H}(36 \mathrm{~B})$ & 109.5 \\
\hline $\mathrm{C}(350)-\mathrm{C}(360)-\mathrm{H}(36 \mathrm{~B})$ & 109.5 \\
\hline $\mathrm{H}(36 \mathrm{~A})-\mathrm{C}(360)-\mathrm{H}(36 \mathrm{~B})$ & 108.1 \\
\hline $\mathrm{C}(370) \# 1-\mathrm{C}(370)-\mathrm{O}(220)$ & $124.9(5)$ \\
\hline $\mathrm{C}(370) \# 1-\mathrm{C}(370)-\mathrm{H}(37 \mathrm{~A})$ & 106.1 \\
\hline $\mathrm{O}(220)-\mathrm{C}(370)-\mathrm{H}(37 \mathrm{~A})$ & 106.1 \\
\hline $\mathrm{C}(370) \# 1-\mathrm{C}(370)-\mathrm{H}(37 \mathrm{~B})$ & 106.1 \\
\hline $\mathrm{O}(220)-\mathrm{C}(370)-\mathrm{H}(37 \mathrm{~B})$ & 106.1 \\
\hline $\mathrm{H}(37 \mathrm{~A})-\mathrm{C}(370)-\mathrm{H}(37 \mathrm{~B})$ & 106.3 \\
\hline $\mathrm{C}(390)-\mathrm{C}(380)-\mathrm{O}(220)$ & $122.3(10)$ \\
\hline $\mathrm{C}(390)-\mathrm{C}(380)-\mathrm{H}(38 \mathrm{~A})$ & 106.8 \\
\hline $\mathrm{O}(220)-\mathrm{C}(380)-\mathrm{H}(38 \mathrm{~A})$ & 106.8 \\
\hline $\mathrm{C}(390)-\mathrm{C}(380)-\mathrm{H}(38 \mathrm{~B})$ & 106.8 \\
\hline $\mathrm{O}(220)-\mathrm{C}(380)-\mathrm{H}(38 \mathrm{~B})$ & 106.8 \\
\hline $\mathrm{H}(38 \mathrm{~A})-\mathrm{C}(380)-\mathrm{H}(38 \mathrm{~B})$ & 106.6 \\
\hline $\mathrm{C}(380)-\mathrm{C}(390)-\mathrm{O}(230)$ & $123.3(10)$ \\
\hline $\mathrm{C}(380)-\mathrm{C}(390)-\mathrm{H}(39 \mathrm{~A})$ & 106.5 \\
\hline $\mathrm{O}(230)-\mathrm{C}(390)-\mathrm{H}(39 \mathrm{~A})$ & 106.5 \\
\hline $\mathrm{C}(380)-\mathrm{C}(390)-\mathrm{H}(39 \mathrm{~B})$ & 106.5 \\
\hline $\mathrm{O}(230)-\mathrm{C}(390)-\mathrm{H}(39 \mathrm{~B})$ & 106.5 \\
\hline $\mathrm{H}(39 \mathrm{~A})-\mathrm{C}(390)-\mathrm{H}(39 \mathrm{~B})$ & 106.5 \\
\hline $\mathrm{C}(2 \mathrm{X})-\mathrm{O}(1 \mathrm{X})-\mathrm{C}(3 \mathrm{X})$ & $113.5(8)$ \\
\hline $\mathrm{O}(1 \mathrm{X})-\mathrm{C}(3 \mathrm{X})-\mathrm{C}(4 \mathrm{X})$ & $110.2(9)$ \\
\hline $\mathrm{O}(1 \mathrm{X})-\mathrm{C}(3 \mathrm{X})-\mathrm{H}(3 \mathrm{XA})$ & 109.6 \\
\hline $\mathrm{C}(4 \mathrm{X})-\mathrm{C}(3 \mathrm{X})-\mathrm{H}(3 \mathrm{XA})$ & 109.6 \\
\hline $\mathrm{O}(1 \mathrm{X})-\mathrm{C}(3 \mathrm{X})-\mathrm{H}(3 \mathrm{XB})$ & 109.6 \\
\hline $\mathrm{C}(4 \mathrm{X})-\mathrm{C}(3 \mathrm{X})-\mathrm{H}(3 \mathrm{XB})$ & 109.6 \\
\hline $\mathrm{H}(3 \mathrm{XA})-\mathrm{C}(3 \mathrm{X})-\mathrm{H}(3 \mathrm{XB})$ & 108.1 \\
\hline $\mathrm{C}(3 \mathrm{X})-\mathrm{C}(4 \mathrm{X})-\mathrm{H}(4 \mathrm{XA})$ & 109.5 \\
\hline $\mathrm{C}(3 \mathrm{X})-\mathrm{C}(4 \mathrm{X})-\mathrm{H}(4 \mathrm{XB})$ & 109.5 \\
\hline $\mathrm{H}(4 \mathrm{XA})-\mathrm{C}(4 \mathrm{X})-\mathrm{H}(4 \mathrm{XB})$ & 109.5 \\
\hline $\mathrm{C}(3 \mathrm{X})-\mathrm{C}(4 \mathrm{X})-\mathrm{H}(4 \mathrm{XC})$ & 109.5 \\
\hline $\mathrm{H}(4 \mathrm{XA})-\mathrm{C}(4 \mathrm{X})-\mathrm{H}(4 \mathrm{XC})$ & 109.5 \\
\hline $\mathrm{H}(4 \mathrm{XB})-\mathrm{C}(4 \mathrm{X})-\mathrm{H}(4 \mathrm{XC})$ & 109.5 \\
\hline
\end{tabular}




\begin{tabular}{|c|c|}
\hline $\mathrm{C}(2 \mathrm{X})-\mathrm{C}(1 \mathrm{X})-\mathrm{H}(1 \mathrm{XA})$ & 109.5 \\
\hline $\mathrm{C}(2 \mathrm{X})-\mathrm{C}(1 \mathrm{X})-\mathrm{H}(1 \mathrm{XB})$ & 109.5 \\
\hline $\mathrm{H}(1 \mathrm{XA})-\mathrm{C}(1 \mathrm{X})-\mathrm{H}(1 \mathrm{XB})$ & 109.5 \\
\hline $\mathrm{C}(2 \mathrm{X})-\mathrm{C}(1 \mathrm{X})-\mathrm{H}(1 \mathrm{XC})$ & 109.5 \\
\hline $\mathrm{H}(1 \mathrm{XA})-\mathrm{C}(1 \mathrm{X})-\mathrm{H}(1 \mathrm{XC})$ & 109.5 \\
\hline $\mathrm{H}(1 \mathrm{XB})-\mathrm{C}(1 \mathrm{X})-\mathrm{H}(1 \mathrm{XC})$ & 109.5 \\
\hline $\mathrm{O}(1 \mathrm{X})-\mathrm{C}(2 \mathrm{X})-\mathrm{C}(1 \mathrm{X})$ & $111.1(9)$ \\
\hline $\mathrm{O}(1 \mathrm{X})-\mathrm{C}(2 \mathrm{X})-\mathrm{H}(2 \mathrm{XA})$ & 109.4 \\
\hline $\mathrm{C}(1 \mathrm{X})-\mathrm{C}(2 \mathrm{X})-\mathrm{H}(2 \mathrm{XA})$ & 109.4 \\
\hline $\mathrm{O}(1 \mathrm{X})-\mathrm{C}(2 \mathrm{X})-\mathrm{H}(2 \mathrm{XB})$ & 109.4 \\
\hline $\mathrm{C}(1 \mathrm{X})-\mathrm{C}(2 \mathrm{X})-\mathrm{H}(2 \mathrm{XB})$ & 109.4 \\
\hline $\mathrm{H}(2 \mathrm{XA})-\mathrm{C}(2 \mathrm{X})-\mathrm{H}(2 \mathrm{XB})$ & 108.0 \\
\hline $\mathrm{C}(400) \# 1-\mathrm{C}(400)-\mathrm{O}(230)$ & $123.4(5)$ \\
\hline $\mathrm{C}(400) \# 1-\mathrm{C}(400)-\mathrm{H}(40 \mathrm{~A})$ & 106.5 \\
\hline $\mathrm{O}(230)-\mathrm{C}(400)-\mathrm{H}(40 \mathrm{~A})$ & 106.5 \\
\hline $\mathrm{C}(400) \# 1-\mathrm{C}(400)-\mathrm{H}(40 \mathrm{~B})$ & 106.5 \\
\hline $\mathrm{O}(230)-\mathrm{C}(400)-\mathrm{H}(40 \mathrm{~B})$ & 106.5 \\
\hline $\mathrm{H}(40 \mathrm{~A})-\mathrm{C}(400)-\mathrm{H}(40 \mathrm{~B})$ & 106.5 \\
\hline $\mathrm{C}(360) \# 1-\mathrm{O}(210)-\mathrm{C}(360)$ & $102(2)$ \\
\hline $\mathrm{C}(360) \# 1-\mathrm{O}(210)-\mathrm{K}(3)$ & $121.3(9)$ \\
\hline $\mathrm{C}(360)-\mathrm{O}(210)-\mathrm{K}(3)$ & $121.3(9)$ \\
\hline $\mathrm{O}(200)-\mathrm{C}(340)-\mathrm{C}(330)$ & $125.3(11)$ \\
\hline $\mathrm{O}(200)-\mathrm{C}(340)-\mathrm{H}(34 \mathrm{~A})$ & 106.0 \\
\hline $\mathrm{C}(330)-\mathrm{C}(340)-\mathrm{H}(34 \mathrm{~A})$ & 106.0 \\
\hline $\mathrm{O}(200)-\mathrm{C}(340)-\mathrm{H}(34 \mathrm{~B})$ & 106.0 \\
\hline $\mathrm{C}(330)-\mathrm{C}(340)-\mathrm{H}(34 \mathrm{~B})$ & 106.0 \\
\hline $\mathrm{H}(34 \mathrm{~A})-\mathrm{C}(340)-\mathrm{H}(34 \mathrm{~B})$ & 106.3 \\
\hline $\mathrm{C}(360)-\mathrm{C}(350)-\mathrm{O}(200)$ & $116.4(16)$ \\
\hline $\mathrm{C}(360)-\mathrm{C}(350)-\mathrm{H}(35 \mathrm{~A})$ & 108.2 \\
\hline $\mathrm{O}(200)-\mathrm{C}(350)-\mathrm{H}(35 \mathrm{~A})$ & 108.2 \\
\hline $\mathrm{C}(360)-\mathrm{C}(350)-\mathrm{H}(35 \mathrm{~B})$ & 108.2 \\
\hline $\mathrm{O}(200)-\mathrm{C}(350)-\mathrm{H}(35 \mathrm{~B})$ & 108.2 \\
\hline$H(35 A)-C(350)-H(35 B)$ & 107.3 \\
\hline$C(6 X)-C(5 X)-O(170)$ & $127.3(16)$ \\
\hline $\mathrm{C}(6 \mathrm{X})-\mathrm{C}(5 \mathrm{X})-\mathrm{H}(5 \mathrm{XA})$ & 105.5 \\
\hline $\mathrm{O}(170)-\mathrm{C}(5 \mathrm{X})-\mathrm{H}(5 \mathrm{XA})$ & 105.5 \\
\hline
\end{tabular}




\begin{tabular}{|c|c|}
\hline $\mathrm{C}(6 \mathrm{X})-\mathrm{C}(5 \mathrm{X})-\mathrm{H}(5 \mathrm{XB})$ & 105.5 \\
\hline $\mathrm{O}(170)-\mathrm{C}(5 \mathrm{X})-\mathrm{H}(5 \mathrm{XB})$ & 105.5 \\
\hline $\mathrm{H}(5 \mathrm{XA})-\mathrm{C}(5 \mathrm{X})-\mathrm{H}(5 \mathrm{XB})$ & 106.1 \\
\hline $\mathrm{C}(5 \mathrm{Y})-\mathrm{C}(6 \mathrm{Y})-\mathrm{O}(160)$ & $123.2(16)$ \\
\hline C(5Y)-C(6Y)-H(6YA) & 106.5 \\
\hline $\mathrm{O}(160)-\mathrm{C}(6 \mathrm{Y})-\mathrm{H}(6 \mathrm{YA})$ & 106.5 \\
\hline $\mathrm{C}(5 \mathrm{Y})-\mathrm{C}(6 \mathrm{Y})-\mathrm{H}(6 \mathrm{YB})$ & 106.5 \\
\hline $\mathrm{O}(160)-\mathrm{C}(6 \mathrm{Y})-\mathrm{H}(6 \mathrm{YB})$ & 106.5 \\
\hline $\mathrm{H}(6 \mathrm{YA})-\mathrm{C}(6 \mathrm{Y})-\mathrm{H}(6 \mathrm{YB})$ & 106.5 \\
\hline$C(8 X)-C(7 X)-O(160)$ & $128.4(17)$ \\
\hline $\mathrm{C}(8 \mathrm{X})-\mathrm{C}(7 \mathrm{X})-\mathrm{H}(7 \mathrm{XA})$ & 105.2 \\
\hline O(160)-C(7X)-H(7XA) & 105.2 \\
\hline $\mathrm{C}(8 \mathrm{X})-\mathrm{C}(7 \mathrm{X})-\mathrm{H}(7 \mathrm{XB})$ & 105.2 \\
\hline $\mathrm{O}(160)-\mathrm{C}(7 \mathrm{X})-\mathrm{H}(7 \mathrm{XB})$ & 105.2 \\
\hline $\mathrm{H}(7 \mathrm{XA})-\mathrm{C}(7 \mathrm{X})-\mathrm{H}(7 \mathrm{XB})$ & 105.9 \\
\hline $\mathrm{C}(7 \mathrm{Y})-\mathrm{C}(8 \mathrm{Y})-\mathrm{O}(150)$ & $129.7(16)$ \\
\hline $\mathrm{C}(7 \mathrm{Y})-\mathrm{C}(8 \mathrm{Y})-\mathrm{H}(8 \mathrm{YA})$ & 104.8 \\
\hline $\mathrm{O}(150)-\mathrm{C}(8 \mathrm{Y})-\mathrm{H}(8 \mathrm{YA})$ & 104.8 \\
\hline $\mathrm{C}(7 \mathrm{Y})-\mathrm{C}(8 \mathrm{Y})-\mathrm{H}(8 \mathrm{YB})$ & 104.8 \\
\hline O(150)-C(8Y)-H(8YB) & 104.8 \\
\hline H(8YA)-C(8Y)-H(8YB) & 105.8 \\
\hline
\end{tabular}

Symmetry transformations used to generate equivalent atoms:

$\# 1 \mathrm{x},-\mathrm{y}+1 / 2, \mathrm{z}$ 
Table S-21. Anisotropic displacement parameters $\left(\AA^{2} \times 10^{3}\right)$ for 4'. The anisotropic displacement factor exponent takes the form: $-2 \pi^{2}\left[h^{2} a^{* 2} U^{11}+\ldots+2 h k a^{*} b^{*} U^{12}\right]$

\begin{tabular}{|c|c|c|c|c|c|c|}
\hline & $\mathrm{U}^{11}$ & $\mathrm{U}^{22}$ & $\mathrm{U}^{33}$ & $\mathrm{U}^{23}$ & $\mathrm{U}^{13}$ & $\mathrm{U}^{12}$ \\
\hline $\mathrm{Fe}(1)$ & $35(1)$ & $39(1)$ & $28(1)$ & $-3(1)$ & $7(1)$ & $-6(1)$ \\
\hline$C(1)$ & $49(5)$ & $48(4)$ & $72(5)$ & $-10(4)$ & $5(4)$ & $-3(3)$ \\
\hline $\mathrm{N}(11)$ & $29(3)$ & $42(3)$ & $22(2)$ & $-1(2)$ & $9(2)$ & $-2(2)$ \\
\hline $\mathrm{N}(21)$ & $29(3)$ & $39(3)$ & $27(2)$ & $-4(2)$ & $2(2)$ & $-1(2)$ \\
\hline $\mathrm{C}(11)$ & $53(4)$ & $47(4)$ & $50(4)$ & $-2(3)$ & $9(3)$ & $1(3)$ \\
\hline $\mathrm{C}(21)$ & 41(4) & $40(3)$ & $24(3)$ & $-2(2)$ & $3(2)$ & $4(3)$ \\
\hline $\mathrm{C}(31)$ & $31(3)$ & $36(3)$ & $29(3)$ & $-4(2)$ & $-5(2)$ & $-3(2)$ \\
\hline $\mathrm{C}(41)$ & $38(3)$ & $41(3)$ & $23(3)$ & $1(2)$ & $1(2)$ & $-5(3)$ \\
\hline $\mathrm{C}(51)$ & $39(4)$ & $51(4)$ & $41(3)$ & $-10(3)$ & $3(3)$ & $-13(3)$ \\
\hline$C(12)$ & $46(4)$ & $38(3)$ & $28(3)$ & $-5(2)$ & $16(3)$ & $-3(3)$ \\
\hline$C(22)$ & $28(3)$ & $41(3)$ & $30(3)$ & $-2(2)$ & $12(2)$ & $2(2)$ \\
\hline $\mathrm{C}(32)$ & $41(4)$ & $45(3)$ & $29(3)$ & $0(2)$ & $10(3)$ & $5(3)$ \\
\hline$C(42)$ & $44(4)$ & $47(4)$ & $47(4)$ & $0(3)$ & $24(3)$ & $-6(3)$ \\
\hline$C(52)$ & $43(4)$ & $50(4)$ & $41(4)$ & $-7(3)$ & $2(3)$ & $-2(3)$ \\
\hline$C(62)$ & $37(4)$ & $42(3)$ & $34(3)$ & $-8(3)$ & $13(3)$ & $-6(3)$ \\
\hline$C(72)$ & $51(4)$ & $51(4)$ & $28(3)$ & $5(3)$ & $11(3)$ & $3(3)$ \\
\hline$C(82)$ & $54(5)$ & $74(5)$ & $44(4)$ & $-2(3)$ & $-8(3)$ & $15(4)$ \\
\hline $\mathrm{C}(92)$ & $56(5)$ & $81(5)$ & $44(4)$ & $-15(4)$ & $3(3)$ & $-6(4)$ \\
\hline$C(102)$ & $42(4)$ & $60(4)$ & $33(3)$ & $-6(3)$ & $-3(3)$ & $-14(3)$ \\
\hline$C(112)$ & $49(4)$ & $90(6)$ & $38(4)$ & $-22(4)$ & $-1(3)$ & $-3(4)$ \\
\hline$C(122)$ & $77(6)$ & $71(5)$ & $45(4)$ & $10(4)$ & $2(4)$ & $1(4)$ \\
\hline$C(13)$ & $23(3)$ & $45(3)$ & $30(3)$ & $-6(2)$ & $-2(2)$ & $-5(2)$ \\
\hline$C(23)$ & $35(3)$ & $54(4)$ & $21(3)$ & $-4(2)$ & $0(2)$ & $-6(3)$ \\
\hline$C(33)$ & $42(4)$ & $62(4)$ & $38(3)$ & $-9(3)$ & $-7(3)$ & $0(3)$ \\
\hline$C(43)$ & $25(3)$ & $64(4)$ & $52(4)$ & $-9(3)$ & $0(3)$ & $2(3)$ \\
\hline$C(53)$ & $31(3)$ & $58(4)$ & $37(3)$ & $-13(3)$ & $13(3)$ & $-11(3)$ \\
\hline$C(63)$ & $26(3)$ & $53(4)$ & $27(3)$ & $-4(3)$ & $9(2)$ & $-5(3)$ \\
\hline$C(73)$ & $53(4)$ & $54(4)$ & $29(3)$ & $-4(3)$ & $-7(3)$ & $5(3)$ \\
\hline$C(83)$ & $84(6)$ & $64(5)$ & $33(4)$ & $0(3)$ & $3(4)$ & $-5(4)$ \\
\hline$C(93)$ & $97(7)$ & $73(5)$ & $35(4)$ & $-5(4)$ & $-26(4)$ & $-15(5)$ \\
\hline$C(103)$ & $50(4)$ & $47(4)$ & $27(3)$ & $1(3)$ & $4(3)$ & $-6(3)$ \\
\hline
\end{tabular}




\begin{tabular}{|c|c|c|c|c|c|c|}
\hline$C(113)$ & $50(4)$ & $61(4)$ & $36(3)$ & $-7(3)$ & $-11(3)$ & $0(3)$ \\
\hline$C(123)$ & $72(6)$ & $75(6)$ & $58(5)$ & $20(4)$ & $6(4)$ & $-12(4)$ \\
\hline $\mathrm{Fe}(2)$ & $61(1)$ & $39(1)$ & $56(1)$ & $8(1)$ & $-28(1)$ & $-9(1)$ \\
\hline$C(2)$ & $107(8)$ & $48(5)$ & $170(12)$ & $18(6)$ & $-56(8)$ & $-16(5)$ \\
\hline $\mathrm{N}(14)$ & $46(3)$ & $53(3)$ & $23(2)$ & $10(2)$ & $-6(2)$ & $-13(2)$ \\
\hline $\mathrm{N}(24)$ & $38(3)$ & $41(3)$ & $30(2)$ & $0(2)$ & $-1(2)$ & $-11(2)$ \\
\hline $\mathrm{C}(14)$ & $57(5)$ & $51(4)$ & $46(4)$ & $-3(3)$ & $-8(3)$ & $-18(3)$ \\
\hline $\mathrm{C}(24)$ & $37(3)$ & $47(3)$ & $26(3)$ & $2(3)$ & $-1(2)$ & $-18(3)$ \\
\hline $\mathrm{C}(34)$ & $44(4)$ & $37(3)$ & $30(3)$ & $-1(2)$ & $4(3)$ & $-8(3)$ \\
\hline $\mathrm{C}(44)$ & $32(3)$ & $41(3)$ & $40(3)$ & $4(3)$ & $5(3)$ & $-1(3)$ \\
\hline $\mathrm{C}(54)$ & $48(4)$ & $42(4)$ & $87(6)$ & $-11(4)$ & $-10(4)$ & $6(3)$ \\
\hline$C(15)$ & $48(4)$ & $42(3)$ & $35(3)$ & $7(3)$ & $-9(3)$ & $-19(3)$ \\
\hline$C(25)$ & $46(4)$ & $42(3)$ & $25(3)$ & $7(2)$ & $-4(3)$ & $-10(3)$ \\
\hline $\mathrm{C}(35)$ & $58(5)$ & $41(3)$ & $34(3)$ & $9(3)$ & $-9(3)$ & $-9(3)$ \\
\hline $\mathrm{C}(45)$ & $45(4)$ & $46(4)$ & $50(4)$ & $6(3)$ & $0(3)$ & $-8(3)$ \\
\hline $\mathrm{C}(55)$ & $33(4)$ & $63(4)$ & $50(4)$ & $3(3)$ & $-2(3)$ & $-5(3)$ \\
\hline$C(65)$ & $46(4)$ & $67(4)$ & $26(3)$ & $5(3)$ & $13(3)$ & $-18(3)$ \\
\hline$C(75)$ & $53(4)$ & $48(4)$ & $32(3)$ & $6(3)$ & $2(3)$ & $-1(3)$ \\
\hline$C(85)$ & $57(5)$ & $63(5)$ & $73(5)$ & $5(4)$ & $27(4)$ & $-6(4)$ \\
\hline$C(95)$ & $77(6)$ & $90(6)$ & $44(4)$ & $-16(4)$ & $6(4)$ & $12(5)$ \\
\hline$C(105)$ & $50(5)$ & $108(7)$ & $25(3)$ & $6(4)$ & $-1(3)$ & $-11(4)$ \\
\hline$C(115)$ & 143(11) & $111(8)$ & $54(5)$ & $-20(5)$ & $15(6)$ & $-38(8)$ \\
\hline$C(125)$ & $51(5)$ & 101(6) & $49(4)$ & $35(4)$ & $19(4)$ & $5(4)$ \\
\hline$C(16)$ & $24(3)$ & $35(3)$ & $47(3)$ & $-5(3)$ & $2(3)$ & $0(2)$ \\
\hline$C(26)$ & $44(4)$ & $51(4)$ & $56(4)$ & $-11(3)$ & $6(3)$ & $-4(3)$ \\
\hline$C(36)$ & $28(4)$ & $49(4)$ & $82(6)$ & $-12(4)$ & $9(3)$ & $-1(3)$ \\
\hline $\mathrm{C}(46)$ & $32(4)$ & $52(4)$ & $83(6)$ & $-22(4)$ & $-10(4)$ & $3(3)$ \\
\hline$C(56)$ & $46(4)$ & $43(4)$ & $56(4)$ & $-10(3)$ & $-13(3)$ & $6(3)$ \\
\hline$C(66)$ & $32(3)$ & $31(3)$ & $42(3)$ & $-6(2)$ & $-3(3)$ & $3(2)$ \\
\hline$C(76)$ & $66(5)$ & $82(6)$ & $61(5)$ & $-15(4)$ & $34(4)$ & $-31(4)$ \\
\hline$C(86)$ & $49(6)$ & 219(14) & $98(8)$ & $-84(9)$ & $43(5)$ & $-38(7)$ \\
\hline $\mathrm{C}(96)$ & $280(20)$ & $108(10)$ & $79(8)$ & $21(7)$ & $-11(11)$ & $-70(12)$ \\
\hline$C(106)$ & $40(4)$ & $45(4)$ & $34(3)$ & $-6(3)$ & $-6(3)$ & $0(3)$ \\
\hline$C(116)$ & $61(5)$ & $53(4)$ & $54(4)$ & $-4(3)$ & $12(4)$ & $1(4)$ \\
\hline$C(126)$ & $85(6)$ & $62(5)$ & $54(5)$ & $10(4)$ & $-10(4)$ & $-6(4)$ \\
\hline $\mathrm{K}(1)$ & $51(1)$ & $40(1)$ & $38(1)$ & $-2(1)$ & $6(1)$ & $2(1)$ \\
\hline
\end{tabular}




\begin{tabular}{|c|c|c|c|c|c|c|}
\hline $\mathrm{O}(10)$ & $72(4)$ & $62(3)$ & $75(4)$ & $0(3)$ & $10(3)$ & $-10(3)$ \\
\hline $\mathrm{O}(20)$ & $70(4)$ & $66(3)$ & $50(3)$ & $-7(2)$ & $-7(3)$ & $5(3)$ \\
\hline $\mathrm{O}(30)$ & $95(5)$ & $53(3)$ & $62(3)$ & $2(3)$ & $-4(3)$ & $12(3)$ \\
\hline $\mathrm{O}(40)$ & $87(4)$ & $51(3)$ & $57(3)$ & $-10(2)$ & $-5(3)$ & $0(3)$ \\
\hline $\mathrm{O}(50)$ & $57(3)$ & $78(4)$ & $43(3)$ & $-7(2)$ & $-9(2)$ & $8(3)$ \\
\hline $\mathrm{O}(60)$ & $106(5)$ & $61(3)$ & $56(3)$ & $9(3)$ & $23(3)$ & $12(3)$ \\
\hline $\mathrm{O}(70)$ & $50(3)$ & $61(3)$ & $48(3)$ & $-6(2)$ & $-4(2)$ & $6(2)$ \\
\hline $\mathrm{O}(80)$ & $83(4)$ & $66(3)$ & $49(3)$ & $3(2)$ & $10(3)$ & $11(3)$ \\
\hline $\mathrm{O}(90)$ & $67(4)$ & $60(3)$ & $73(4)$ & $-4(3)$ & $21(3)$ & $2(3)$ \\
\hline $\mathrm{O}(100)$ & $64(4)$ & $80(4)$ & $63(3)$ & $-12(3)$ & $3(3)$ & $-2(3)$ \\
\hline $\mathrm{C}(10)$ & $61(6)$ & $67(6)$ & $123(9)$ & $-18(6)$ & $-10(6)$ & $-15(5)$ \\
\hline $\mathrm{C}(20)$ & $80(7)$ & $84(7)$ & $85(7)$ & $-11(5)$ & $-25(5)$ & $-3(5)$ \\
\hline $\mathrm{C}(30)$ & $80(6)$ & $94(7)$ & $44(4)$ & $9(4)$ & $-11(4)$ & $7(5)$ \\
\hline $\mathrm{C}(40)$ & $65(5)$ & $70(5)$ & $53(4)$ & $12(4)$ & $-9(4)$ & $12(4)$ \\
\hline$C(50)$ & $76(6)$ & 41(4) & $84(6)$ & $14(4)$ & $13(5)$ & $10(4)$ \\
\hline $\mathrm{C}(60)$ & $89(7)$ & $38(4)$ & $76(5)$ & $5(4)$ & $5(5)$ & $-6(4)$ \\
\hline$C(70)$ & $76(6)$ & $66(5)$ & $73(6)$ & $-21(4)$ & $3(5)$ & $-2(5)$ \\
\hline$C(80)$ & $73(6)$ & $83(6)$ & $48(4)$ & $-24(4)$ & $-4(4)$ & $-7(5)$ \\
\hline $\mathrm{C}(90)$ & $98(7)$ & $79(6)$ & $50(5)$ & $4(4)$ & $10(5)$ & $22(5)$ \\
\hline$C(100)$ & $98(7)$ & $77(6)$ & $39(4)$ & $15(4)$ & $20(4)$ & $31(5)$ \\
\hline$C(110)$ & $111(8)$ & $55(5)$ & $76(6)$ & $12(4)$ & $16(6)$ & $5(5)$ \\
\hline$C(120)$ & $93(7)$ & $66(6)$ & $90(7)$ & $13(5)$ & $25(6)$ & $-27(5)$ \\
\hline$C(130)$ & $64(5)$ & $63(5)$ & $51(4)$ & $-19(4)$ & $-9(4)$ & $10(4)$ \\
\hline$C(140)$ & $89(6)$ & $70(5)$ & $48(4)$ & $-12(4)$ & $12(4)$ & $13(5)$ \\
\hline$C(150)$ & $95(7)$ & $80(6)$ & $53(5)$ & $16(4)$ & $-1(5)$ & $9(5)$ \\
\hline$C(160)$ & $87(7)$ & $77(6)$ & $78(6)$ & $17(5)$ & $43(5)$ & $12(5)$ \\
\hline$C(170)$ & $52(5)$ & $76(6)$ & $101(7)$ & $-16(5)$ & $16(5)$ & $-13(4)$ \\
\hline$C(180)$ & $70(6)$ & $101(8)$ & $81(6)$ & $-7(6)$ & $4(5)$ & $-8(6)$ \\
\hline$C(190)$ & $71(6)$ & $67(5)$ & $58(5)$ & $-6(4)$ & $-17(4)$ & $3(4)$ \\
\hline$C(200)$ & $53(5)$ & $69(5)$ & $61(5)$ & $-9(4)$ & $-2(4)$ & $12(4)$ \\
\hline $\mathrm{K}(2)$ & $34(1)$ & $38(1)$ & $34(1)$ & 0 & $-1(1)$ & 0 \\
\hline $\mathrm{O}(110)$ & $56(5)$ & $86(5)$ & $35(3)$ & 0 & $6(3)$ & 0 \\
\hline $\mathrm{O}(120)$ & $55(3)$ & $56(3)$ & $65(3)$ & $-22(2)$ & $0(3)$ & $-7(2)$ \\
\hline $\mathrm{O}(130)$ & $59(3)$ & $43(3)$ & $56(3)$ & $-4(2)$ & $2(2)$ & $-6(2)$ \\
\hline $\mathrm{O}(140)$ & $44(4)$ & $58(4)$ & $34(3)$ & 0 & $4(3)$ & 0 \\
\hline $\mathrm{O}(150)$ & $39(4)$ & $103(5)$ & $36(3)$ & 0 & $2(3)$ & 0 \\
\hline
\end{tabular}




\begin{tabular}{|c|c|c|c|c|c|c|}
\hline$O(160)$ & $43(3)$ & $53(3)$ & $61(3)$ & $-3(2)$ & $-2(2)$ & $4(2)$ \\
\hline$C(210)$ & $83(7)$ & $109(7)$ & $43(4)$ & $-25(5)$ & $3(4)$ & $-3(5)$ \\
\hline$C(220)$ & $80(7)$ & $87(6)$ & $65(5)$ & $-26(5)$ & $0(5)$ & $-15(5)$ \\
\hline$C(230)$ & $59(5)$ & $39(4)$ & $99(6)$ & $-11(4)$ & $-2(5)$ & $-2(3)$ \\
\hline$C(240)$ & $67(5)$ & $43(4)$ & $77(5)$ & $-3(4)$ & $6(4)$ & $-10(4)$ \\
\hline$C(250)$ & $47(4)$ & $56(4)$ & $67(5)$ & $19(4)$ & $9(4)$ & $-12(3)$ \\
\hline$C(260)$ & $59(5)$ & $66(5)$ & $46(4)$ & $13(4)$ & $14(3)$ & $-4(4)$ \\
\hline $\mathrm{C}(8 \mathrm{X})$ & $80(12)$ & $152(10)$ & $138(19)$ & $50(11)$ & $54(13)$ & $47(9)$ \\
\hline$C(7 Y)$ & $39(6)$ & $65(8)$ & $58(7)$ & $3(6)$ & $-4(5)$ & $0(5)$ \\
\hline$C(6 X)$ & $47(7)$ & $67(8)$ & $55(6)$ & $1(5)$ & $-4(5)$ & $4(6)$ \\
\hline $\mathrm{C}(5 \mathrm{Y})$ & $97(14)$ & $120(9)$ & $42(8)$ & $-7(6)$ & $-14(8)$ & $43(9)$ \\
\hline $\mathrm{O}(170)$ & $42(4)$ & $84(4)$ & $42(4)$ & 0 & $4(3)$ & 0 \\
\hline $\mathrm{K}(3)$ & $40(1)$ & $60(1)$ & $36(1)$ & 0 & $4(1)$ & 0 \\
\hline $\mathrm{O}(180)$ & $69(5)$ & $93(5)$ & $57(5)$ & 0 & $20(4)$ & 0 \\
\hline $\mathrm{O}(190)$ & $235(10)$ & $81(4)$ & $78(4)$ & $6(3)$ & $44(5)$ & $76(5)$ \\
\hline $\mathrm{O}(200)$ & $336(17)$ & $246(11)$ & 214(9) & $178(8)$ & $167(11)$ & $190(12)$ \\
\hline $\mathrm{O}(220)$ & $69(4)$ & $85(4)$ & $57(3)$ & $-7(3)$ & $-5(3)$ & $-8(3)$ \\
\hline $\mathrm{O}(230)$ & $65(4)$ & $79(4)$ & $61(3)$ & $-4(3)$ & $8(3)$ & $-14(3)$ \\
\hline$C(310)$ & 201(10) & $131(6)$ & $99(6)$ & $7(4)$ & $54(6)$ & $66(6)$ \\
\hline$C(320)$ & 201(10) & $131(6)$ & $99(6)$ & $7(4)$ & $54(6)$ & $66(6)$ \\
\hline$C(330)$ & $86(7)$ & $73(6)$ & $149(8)$ & $32(5)$ & $-4(6)$ & $4(5)$ \\
\hline$C(360)$ & $162(14)$ & $409(18)$ & 192(13) & 101(10) & $100(11)$ & $101(12)$ \\
\hline$C(370)$ & $215(14)$ & 111(7) & $60(5)$ & $-12(5)$ & $-36(7)$ & $-7(7)$ \\
\hline$C(380)$ & $164(11)$ & $120(8)$ & $94(6)$ & $-36(5)$ & $37(6)$ & $-70(8)$ \\
\hline$C(390)$ & $137(9)$ & $131(8)$ & $77(5)$ & $-25(5)$ & $18(5)$ & $-64(7)$ \\
\hline $\mathrm{O}(1 \mathrm{X})$ & $70(4)$ & $95(5)$ & $74(4)$ & $-11(3)$ & $13(3)$ & $6(4)$ \\
\hline$C(3 X)$ & $76(7)$ & $106(8)$ & $77(6)$ & $13(6)$ & $-1(5)$ & $-1(6)$ \\
\hline$C(4 X)$ & $81(7)$ & $99(7)$ & $65(6)$ & $4(5)$ & $-2(5)$ & $10(6)$ \\
\hline$C(1 X)$ & $112(9)$ & $89(8)$ & $125(10)$ & $-20(7)$ & $46(8)$ & $-20(7)$ \\
\hline$C(2 X)$ & $68(6)$ & $134(10)$ & $77(6)$ & $6(6)$ & $31(5)$ & $-3(6)$ \\
\hline$C(400)$ & $115(8)$ & $112(8)$ & 204(13) & $11(7)$ & 101(9) & $-6(6)$ \\
\hline $\mathrm{O}(210)$ & $103(10)$ & $321(17)$ & $77(8)$ & 0 & $8(8)$ & 0 \\
\hline$C(340)$ & $142(11)$ & $96(8)$ & $137(8)$ & $44(6)$ & $2(7)$ & $4(7)$ \\
\hline$C(350)$ & $228(17)$ & $306(15)$ & $152(10)$ & $150(9)$ & $92(11)$ & $110(11)$ \\
\hline$C(5 X)$ & $102(14)$ & $124(9)$ & $34(7)$ & $16(6)$ & $-2(7)$ & $52(9)$ \\
\hline $\mathrm{C}(6 \mathrm{Y})$ & $54(9)$ & $85(8)$ & $50(6)$ & $9(5)$ & $-4(5)$ & $24(7)$ \\
\hline
\end{tabular}




\begin{tabular}{lcccccc}
$\mathrm{C}(7 \mathrm{X})$ & $28(7)$ & $90(8)$ & $58(6)$ & $-3(5)$ & $-14(5)$ & $22(6)$ \\
$\mathrm{C}(8 \mathrm{Y})$ & $94(15)$ & $152(10)$ & $62(8)$ & $33(7)$ & $46(9)$ & $68(10)$ \\
\hline
\end{tabular}


Table S-22. Hydrogen coordinates ( $\left.\times 10^{4}\right)$ and isotropic displacement parameters $\left(\AA^{2} \times 10^{3}\right)$ for 4 '.

\begin{tabular}{|c|c|c|c|c|}
\hline & $\mathrm{x}$ & $\mathrm{y}$ & z & $\mathrm{U}(\mathrm{eq})$ \\
\hline $\mathrm{H}(1 \mathrm{~A})$ & -1957 & 3451 & 4726 & 85 \\
\hline $\mathrm{H}(1 \mathrm{~B})$ & -733 & 3396 & 4641 & 85 \\
\hline $\mathrm{H}(1 \mathrm{C})$ & -1400 & 3505 & 3984 & 85 \\
\hline $\mathrm{H}(11 \mathrm{G})$ & -2955 & 4639 & 5382 & 75 \\
\hline $\mathrm{H}(11 \mathrm{H})$ & -2007 & 4811 & 5705 & 75 \\
\hline $\mathrm{H}(11 \mathrm{I})$ & -2518 & 4570 & 6155 & 75 \\
\hline $\mathrm{H}(31)$ & -463 & 4714 & 5248 & 38 \\
\hline $\mathrm{H}(51 \mathrm{~A})$ & 1859 & 4438 & 5042 & 65 \\
\hline $\mathrm{H}(51 \mathrm{~B})$ & 1223 & 4719 & 5041 & 65 \\
\hline $\mathrm{H}(51 \mathrm{C})$ & 1445 & 4564 & 4313 & 65 \\
\hline $\mathrm{H}(32)$ & -3445 & 3844 & 7199 & 46 \\
\hline $\mathrm{H}(42)$ & -4930 & 3717 & 6586 & 55 \\
\hline $\mathrm{H}(52)$ & -5108 & 3834 & 5396 & 54 \\
\hline $\mathrm{H}(72)$ & -1209 & 4183 & 6469 & 52 \\
\hline $\mathrm{H}(82 \mathrm{~A})$ & -1412 & 3668 & 7170 & 86 \\
\hline $\mathrm{H}(82 \mathrm{~B})$ & -338 & 3827 & 7049 & 86 \\
\hline $\mathrm{H}(82 \mathrm{C})$ & -955 & 3702 & 6389 & 86 \\
\hline $\mathrm{H}(92 \mathrm{~A})$ & -2124 & 4371 & 7417 & 91 \\
\hline $\mathrm{H}(92 \mathrm{~B})$ & -1106 & 4219 & 7708 & 91 \\
\hline $\mathrm{H}(92 \mathrm{C})$ & -2227 & 4078 & 7780 & 91 \\
\hline $\mathrm{H}(102)$ & -3215 & 4189 & 4357 & 54 \\
\hline $\mathrm{H}(11 \mathrm{~J})$ & -3312 & 3724 & 4087 & 88 \\
\hline $\mathrm{H}(11 \mathrm{~K})$ & -4058 & 3889 & 3560 & 88 \\
\hline $\mathrm{H}(11 \mathrm{~L})$ & -4548 & 3725 & 4210 & 88 \\
\hline $\mathrm{H}(12 \mathrm{G})$ & -5439 & 4170 & 4522 & 97 \\
\hline $\mathrm{H}(12 \mathrm{H})$ & -4827 & 4335 & 3923 & 97 \\
\hline $\mathrm{H}(12 \mathrm{I})$ & -4747 & 4429 & 4731 & 97 \\
\hline $\mathrm{H}(33)$ & 3045 & 3582 & 4982 & 57 \\
\hline $\mathrm{H}(43)$ & 3549 & 3628 & 3838 & 56 \\
\hline $\mathrm{H}(53)$ & 2584 & 3894 & 3060 & 50 \\
\hline
\end{tabular}




\begin{tabular}{|c|c|c|c|c|}
\hline $\mathrm{H}(73)$ & 791 & 3897 & 5771 & 54 \\
\hline $\mathrm{H}(83 \mathrm{~A})$ & 781 & 3418 & 5570 & 91 \\
\hline $\mathrm{H}(83 \mathrm{~B})$ & 951 & 3492 & 6385 & 91 \\
\hline $\mathrm{H}(83 \mathrm{C})$ & 1922 & 3399 & 5913 & 91 \\
\hline $\mathrm{H}(93 \mathrm{~A})$ & 2892 & 3823 & 6200 & 103 \\
\hline H(93B) & 1959 & 3938 & 6675 & 103 \\
\hline $\mathrm{H}(93 \mathrm{C})$ & 2395 & 4114 & 6029 & 103 \\
\hline $\mathrm{H}(103)$ & 350 & 4295 & 3520 & 50 \\
\hline $\mathrm{H}(11 \mathrm{M})$ & 550 & 3857 & 2532 & 74 \\
\hline $\mathrm{H}(11 \mathrm{~N})$ & -328 & 4089 & 2493 & 74 \\
\hline $\mathrm{H}(110)$ & -326 & 3876 & 3132 & 74 \\
\hline $\mathrm{H}(12 \mathrm{~J})$ & 1935 & 4468 & 3036 & 103 \\
\hline $\mathrm{H}(12 \mathrm{~K})$ & 1014 & 4467 & 2462 & 103 \\
\hline $\mathrm{H}(12 \mathrm{~L})$ & 1899 & 4237 & 2442 & 103 \\
\hline $\mathrm{H}(0 \mathrm{AA})$ & 3440 & 4100 & 10500 & 163 \\
\hline $\mathrm{H}(0 \mathrm{AB})$ & 4348 & 4141 & 9931 & 163 \\
\hline $\mathrm{H}(0 \mathrm{AC})$ & 4636 & 4096 & 10746 & 163 \\
\hline $\mathrm{H}(14 \mathrm{~A})$ & 1870 & 2968 & 9669 & 77 \\
\hline $\mathrm{H}(14 \mathrm{~B})$ & 2732 & 2736 & 9547 & 77 \\
\hline $\mathrm{H}(14 \mathrm{C})$ & 2518 & 2959 & 8947 & 77 \\
\hline $\mathrm{H}(34)$ & 4232 & 2793 & 10060 & 44 \\
\hline $\mathrm{H}(54 \mathrm{~A})$ & 6611 & 2960 & 10514 & 88 \\
\hline $\mathrm{H}(54 \mathrm{~B})$ & 5768 & 2723 & 10620 & 88 \\
\hline $\mathrm{H}(54 \mathrm{C})$ & 5988 & 2936 & 11243 & 88 \\
\hline $\mathrm{H}(35)$ & 1614 & 3599 & 7758 & 53 \\
\hline $\mathrm{H}(45)$ & 113 & 3748 & 8307 & 57 \\
\hline $\mathrm{H}(55)$ & 103 & 3783 & 9557 & 58 \\
\hline $\mathrm{H}(75)$ & 3800 & 3288 & 8564 & 53 \\
\hline $\mathrm{H}(85 \mathrm{~A})$ & 3755 & 3805 & 7870 & 96 \\
\hline $\mathrm{H}(85 \mathrm{~B})$ & 4771 & 3617 & 7924 & 96 \\
\hline $\mathrm{H}(85 \mathrm{C})$ & 4278 & 3747 & 8623 & 96 \\
\hline $\mathrm{H}(95 \mathrm{~A})$ & 2820 & 3106 & 7635 & 105 \\
\hline H(95B) & 3918 & 3216 & 7352 & 105 \\
\hline $\mathrm{H}(95 \mathrm{C})$ & 2878 & 3390 & 7222 & 105 \\
\hline $\mathrm{H}(105)$ & 2184 & 3576 & 10704 & 73 \\
\hline $\mathrm{H}(11 \mathrm{~A})$ & 1541 & 4032 & 10588 & 154 \\
\hline
\end{tabular}




\begin{tabular}{|c|c|c|c|c|}
\hline $\mathrm{H}(11 \mathrm{~B})$ & 1167 & 3891 & 11307 & 154 \\
\hline $\mathrm{H}(11 \mathrm{C})$ & 376 & 3917 & 10655 & 154 \\
\hline $\mathrm{H}(12 \mathrm{~A})$ & 46 & 3417 & 10571 & 100 \\
\hline $\mathrm{H}(12 \mathrm{~B})$ & 672 & 3411 & 11303 & 100 \\
\hline $\mathrm{H}(12 \mathrm{C})$ & 1014 & 3215 & 10670 & 100 \\
\hline $\mathrm{H}(36)$ & 8390 & 3735 & 10671 & 64 \\
\hline $\mathrm{H}(46)$ & 8401 & 3745 & 11892 & 67 \\
\hline $\mathrm{H}(56)$ & 6941 & 3584 & 12513 & 58 \\
\hline $\mathrm{H}(76)$ & 6362 & 3446 & 9593 & 84 \\
\hline $\mathrm{H}(86 \mathrm{~A})$ & 7920 & 3385 & 8959 & 183 \\
\hline $\mathrm{H}(86 \mathrm{~B})$ & 7962 & 3214 & 9678 & 183 \\
\hline $\mathrm{H}(86 \mathrm{C})$ & 8585 & 3495 & 9616 & 183 \\
\hline $\mathrm{H}(96 \mathrm{~A})$ & 6278 & 3924 & 9571 & 233 \\
\hline $\mathrm{H}(96 \mathrm{~B})$ & 6901 & 3817 & 8894 & 233 \\
\hline $\mathrm{H}(96 \mathrm{C})$ & 7525 & 3943 & 9550 & 233 \\
\hline $\mathrm{H}(106)$ & 4715 & 3263 & 11729 & 48 \\
\hline $\mathrm{H}(11 \mathrm{D})$ & 4752 & 3751 & 12548 & 84 \\
\hline $\mathrm{H}(11 \mathrm{E})$ & 3750 & 3562 & 12422 & 84 \\
\hline $\mathrm{H}(11 \mathrm{~F})$ & 4244 & 3729 & 11777 & 84 \\
\hline $\mathrm{H}(12 \mathrm{D})$ & 5776 & 3076 & 12636 & 101 \\
\hline $\mathrm{H}(12 \mathrm{E})$ & 4677 & 3174 & 12946 & 101 \\
\hline $\mathrm{H}(12 \mathrm{~F})$ & 5701 & 3355 & 13068 & 101 \\
\hline $\mathrm{H}(10 \mathrm{~A})$ & 371 & 4618 & 7642 & 101 \\
\hline $\mathrm{H}(10 \mathrm{~B})$ & 723 & 4349 & 7226 & 101 \\
\hline $\mathrm{H}(20 \mathrm{~A})$ & 1789 & 4615 & 6518 & 99 \\
\hline $\mathrm{H}(20 \mathrm{~B})$ & 578 & 4689 & 6435 & 99 \\
\hline $\mathrm{H}(30 \mathrm{~A})$ & 887 & 5138 & 6066 & 87 \\
\hline $\mathrm{H}(30 \mathrm{~B})$ & 2126 & 5091 & 6085 & 87 \\
\hline $\mathrm{H}(40 \mathrm{C})$ & 1691 & 5551 & 6220 & 75 \\
\hline $\mathrm{H}(40 \mathrm{D})$ & 1066 & 5477 & 6927 & 75 \\
\hline $\mathrm{H}(50 \mathrm{~A})$ & 2155 & 5728 & 7680 & 81 \\
\hline $\mathrm{H}(50 \mathrm{~B})$ & 2698 & 5841 & 6976 & 81 \\
\hline $\mathrm{H}(60 \mathrm{~A})$ & 4329 & 5669 & 7376 & 81 \\
\hline $\mathrm{H}(60 \mathrm{~B})$ & 3873 & 5892 & 7907 & 81 \\
\hline $\mathrm{H}(70 \mathrm{~A})$ & 2610 & 5624 & 8867 & 86 \\
\hline $\mathrm{H}(70 \mathrm{~B})$ & 3663 & 5790 & 9038 & 86 \\
\hline
\end{tabular}




\begin{tabular}{|c|c|c|c|c|}
\hline $\mathrm{H}(80 \mathrm{~A})$ & 4418 & 5371 & 9470 & 81 \\
\hline $\mathrm{H}(80 \mathrm{~B})$ & 3465 & 5476 & 9946 & 81 \\
\hline $\mathrm{H}(90 \mathrm{~A})$ & 3198 & 5017 & 10314 & 91 \\
\hline $\mathrm{H}(90 \mathrm{~B})$ & 4128 & 4917 & 9811 & 91 \\
\hline $\mathrm{H}(10 \mathrm{C})$ & 2888 & 4568 & 10060 & 85 \\
\hline $\mathrm{H}(10 \mathrm{D})$ & 2027 & 4741 & 9640 & 85 \\
\hline $\mathrm{H}(11 \mathrm{P})$ & 2511 & 4229 & 9266 & 97 \\
\hline $\mathrm{H}(11 \mathrm{Q})$ & 3139 & 4234 & 8536 & 97 \\
\hline $\mathrm{H}(12 \mathrm{M})$ & 1350 & 4198 & 8356 & 100 \\
\hline $\mathrm{H}(12 \mathrm{~N})$ & 1184 & 4484 & 8746 & 100 \\
\hline $\mathrm{H}(13 \mathrm{~A})$ & 3303 & 4345 & 6987 & 72 \\
\hline $\mathrm{H}(13 \mathrm{~B})$ & 4362 & 4172 & 6961 & 72 \\
\hline $\mathrm{H}(14 \mathrm{D})$ & 5217 & 4539 & 6395 & 83 \\
\hline $\mathrm{H}(14 \mathrm{E})$ & 4223 & 4443 & 5940 & 83 \\
\hline $\mathrm{H}(15 \mathrm{~A})$ & 4151 & 5160 & 6046 & 91 \\
\hline $\mathrm{H}(15 \mathrm{~B})$ & 4835 & 4933 & 5656 & 91 \\
\hline $\mathrm{H}(16 \mathrm{~A})$ & 6009 & 4926 & 6620 & 97 \\
\hline $\mathrm{H}(16 \mathrm{~B})$ & 5934 & 5226 & 6289 & 97 \\
\hline $\mathrm{H}(17 \mathrm{~A})$ & 5710 & 5355 & 8080 & 91 \\
\hline $\mathrm{H}(17 \mathrm{~B})$ & 6579 & 5330 & 7478 & 91 \\
\hline $\mathrm{H}(18 \mathrm{~A})$ & 6658 & 4848 & 7682 & 101 \\
\hline $\mathrm{H}(18 \mathrm{~B})$ & 6979 & 5017 & 8376 & 101 \\
\hline $\mathrm{H}(19 \mathrm{~A})$ & 5209 & 4506 & 8902 & 79 \\
\hline $\mathrm{H}(19 \mathrm{~B})$ & 6398 & 4528 & 8652 & 79 \\
\hline $\mathrm{H}(20 \mathrm{C})$ & 5893 & 4455 & 7465 & 73 \\
\hline $\mathrm{H}(20 \mathrm{D})$ & 5465 & 4209 & 7945 & 73 \\
\hline $\mathrm{H}(21 \mathrm{~A})$ & 1545 & 2744 & 15152 & 94 \\
\hline $\mathrm{H}(21 \mathrm{~B})$ & 378 & 2743 & 14843 & 94 \\
\hline $\mathrm{H}(22 \mathrm{~A})$ & 1285 & 3148 & 14566 & 93 \\
\hline $\mathrm{H}(22 \mathrm{~B})$ & 2109 & 2971 & 14123 & 93 \\
\hline $\mathrm{H}(23 \mathrm{~A})$ & 863 & 3393 & 13609 & 78 \\
\hline $\mathrm{H}(23 \mathrm{~B})$ & 162 & 3261 & 12997 & 78 \\
\hline $\mathrm{H}(24 \mathrm{~A})$ & 1788 & 3401 & 12549 & 75 \\
\hline $\mathrm{H}(24 \mathrm{~B})$ & 2371 & 3202 & 13095 & 75 \\
\hline $\mathrm{H}(25 \mathrm{~A})$ & 3105 & 2954 & 12166 & 68 \\
\hline $\mathrm{H}(25 \mathrm{~B})$ & 2546 & 3147 & 11597 & 68 \\
\hline
\end{tabular}




\begin{tabular}{|c|c|c|c|c|}
\hline $\mathrm{H}(26 \mathrm{~A})$ & 1644 & 2756 & 11146 & 68 \\
\hline $\mathrm{H}(26 \mathrm{~B})$ & 2887 & 2733 & 11050 & 68 \\
\hline $\mathrm{H}(8 \mathrm{XA})$ & -2877 & 2700 & 13291 & 148 \\
\hline $\mathrm{H}(8 \mathrm{XB})$ & -2340 & 2815 & 13999 & 148 \\
\hline H(7YA) & -2271 & 3103 & 12846 & 65 \\
\hline H(7YB) & -2593 & 2818 & 12478 & 65 \\
\hline $\mathrm{H}(6 \mathrm{XA})$ & -2344 & 2781 & 11954 & 68 \\
\hline $\mathrm{H}(6 \mathrm{XB})$ & -1902 & 3080 & 11757 & 68 \\
\hline H(5YA) & -1001 & 2800 & 10769 & 104 \\
\hline $\mathrm{H}(5 \mathrm{YB})$ & -1953 & 2685 & 11231 & 104 \\
\hline $\mathrm{H}(31 \mathrm{~A})$ & 5607 & 2730 & 9034 & 173 \\
\hline $\mathrm{H}(31 \mathrm{~B})$ & 6769 & 2836 & 9100 & 173 \\
\hline $\mathrm{H}(32 \mathrm{~A})$ & 6168 & 3135 & 8532 & 173 \\
\hline $\mathrm{H}(32 \mathrm{~B})$ & 5299 & 2948 & 8169 & 173 \\
\hline $\mathrm{H}(33 \mathrm{~A})$ & 6720 & 3332 & 7525 & 123 \\
\hline $\mathrm{H}(33 \mathrm{~B})$ & 5779 & 3184 & 7125 & 123 \\
\hline $\mathrm{H}(36 \mathrm{~A})$ & 7322 & 2691 & 5304 & 305 \\
\hline $\mathrm{H}(36 \mathrm{~B})$ & 8492 & 2715 & 5012 & 305 \\
\hline $\mathrm{H}(37 \mathrm{~A})$ & 10076 & 2686 & 9262 & 155 \\
\hline $\mathrm{H}(37 \mathrm{~B})$ & 8905 & 2686 & 9530 & 155 \\
\hline $\mathrm{H}(38 \mathrm{~A})$ & 9505 & 3155 & 8435 & 151 \\
\hline $\mathrm{H}(38 \mathrm{~B})$ & 10403 & 2957 & 8699 & 151 \\
\hline $\mathrm{H}(39 \mathrm{~A})$ & 10986 & 2954 & 7773 & 138 \\
\hline H(39B) & 10090 & 3153 & 7510 & 138 \\
\hline $\mathrm{H}(3 \mathrm{XA})$ & -614 & 5208 & 9348 & 104 \\
\hline $\mathrm{H}(3 \mathrm{XB})$ & -1007 & 5124 & 8573 & 104 \\
\hline $\mathrm{H}(4 \mathrm{XA})$ & 1076 & 5225 & 8950 & 123 \\
\hline $\mathrm{H}(4 \mathrm{XB})$ & 354 & 5424 & 8493 & 123 \\
\hline $\mathrm{H}(4 \mathrm{XC})$ & 697 & 5135 & 8178 & 123 \\
\hline $\mathrm{H}(1 \mathrm{XA})$ & -759 & 4299 & 9148 & 163 \\
\hline $\mathrm{H}(1 \mathrm{XB})$ & -1322 & 4339 & 9894 & 163 \\
\hline $\mathrm{H}(1 \mathrm{XC})$ & -108 & 4409 & 9811 & 163 \\
\hline $\mathrm{H}(2 \mathrm{XA})$ & -1655 & 4697 & 9041 & 112 \\
\hline $\mathrm{H}(2 \mathrm{XB})$ & -1276 & 4800 & 9803 & 112 \\
\hline $\mathrm{H}(40 \mathrm{~A})$ & 10800 & 2691 & 6431 & 172 \\
\hline $\mathrm{H}(40 \mathrm{~B})$ & 11372 & 2691 & 7175 & 172 \\
\hline
\end{tabular}




\begin{tabular}{lrrrr}
$\mathrm{H}(34 \mathrm{~A})$ & 6594 & 3180 & 6264 & 150 \\
$\mathrm{H}(34 \mathrm{~B})$ & 7532 & 3325 & 6662 & 150 \\
$\mathrm{H}(35 \mathrm{~A})$ & 7927 & 3101 & 5515 & 274 \\
$\mathrm{H}(35 \mathrm{~B})$ & 8949 & 2981 & 5883 & 274 \\
$\mathrm{H}(5 \mathrm{XA})$ & -411 & 2902 & 11407 & 104 \\
$\mathrm{H}(5 \mathrm{XB})$ & -1359 & 2792 & 10953 & 104 \\
$\mathrm{H}(6 \mathrm{YA})$ & -1596 & 3073 & 11627 & 76 \\
$\mathrm{H}(6 \mathrm{YB})$ & -375 & 3019 & 11601 & 76 \\
$\mathrm{H}(7 \mathrm{X} A)$ & -1236 & 3022 & 13483 & 71 \\
$\mathrm{H}(7 X B)$ & -2233 & 3063 & 13002 & 71 \\
$\mathrm{H}(8 Y A)$ & -2609 & 2786 & 13647 & 123 \\
$\mathrm{H}(8 \mathrm{YB})$ & -1457 & 2899 & 13687 & 123 \\
& & & & \\
\hline
\end{tabular}


Table S-23. Torsion angles $\left[^{\circ}\right]$ for $\mathbf{4}^{\prime}$.

\begin{tabular}{|c|c|}
\hline $\mathrm{C}(12)-\mathrm{N}(11)-\mathrm{C}(21)-\mathrm{C}(31)$ & $163.1(5)$ \\
\hline $\mathrm{Fe}(1)-\mathrm{N}(11)-\mathrm{C}(21)-\mathrm{C}(31)$ & $-6.4(8)$ \\
\hline $\mathrm{C}(12)-\mathrm{N}(11)-\mathrm{C}(21)-\mathrm{C}(11)$ & $-16.5(8)$ \\
\hline $\mathrm{Fe}(1)-\mathrm{N}(11)-\mathrm{C}(21)-\mathrm{C}(11)$ & $174.0(4)$ \\
\hline $\mathrm{N}(11)-\mathrm{C}(21)-\mathrm{C}(31)-\mathrm{C}(41)$ & $-1.9(10)$ \\
\hline $\mathrm{C}(11)-\mathrm{C}(21)-\mathrm{C}(31)-\mathrm{C}(41)$ & $177.7(6)$ \\
\hline $\mathrm{C}(13)-\mathrm{N}(21)-\mathrm{C}(41)-\mathrm{C}(31)$ & $-176.7(5)$ \\
\hline $\mathrm{Fe}(1)-\mathrm{N}(21)-\mathrm{C}(41)-\mathrm{C}(31)$ & $1.0(8)$ \\
\hline $\mathrm{C}(13)-\mathrm{N}(21)-\mathrm{C}(41)-\mathrm{C}(51)$ & $2.4(8)$ \\
\hline $\mathrm{Fe}(1)-\mathrm{N}(21)-\mathrm{C}(41)-\mathrm{C}(51)$ & $-179.9(4)$ \\
\hline $\mathrm{C}(21)-\mathrm{C}(31)-\mathrm{C}(41)-\mathrm{N}(21)$ & $5.0(10)$ \\
\hline$C(21)-C(31)-C(41)-C(51)$ & $-174.2(6)$ \\
\hline $\mathrm{C}(21)-\mathrm{N}(11)-\mathrm{C}(12)-\mathrm{C}(62)$ & $109.2(7)$ \\
\hline $\mathrm{Fe}(1)-\mathrm{N}(11)-\mathrm{C}(12)-\mathrm{C}(62)$ & $-80.6(6)$ \\
\hline $\mathrm{C}(21)-\mathrm{N}(11)-\mathrm{C}(12)-\mathrm{C}(22)$ & $-77.4(7)$ \\
\hline $\mathrm{Fe}(1)-\mathrm{N}(11)-\mathrm{C}(12)-\mathrm{C}(22)$ & $92.8(5)$ \\
\hline $\mathrm{C}(62)-\mathrm{C}(12)-\mathrm{C}(22)-\mathrm{C}(32)$ & $0.1(9)$ \\
\hline $\mathrm{N}(11)-\mathrm{C}(12)-\mathrm{C}(22)-\mathrm{C}(32)$ & $-173.4(5)$ \\
\hline $\mathrm{C}(62)-\mathrm{C}(12)-\mathrm{C}(22)-\mathrm{C}(72)$ & $177.9(6)$ \\
\hline $\mathrm{N}(11)-\mathrm{C}(12)-\mathrm{C}(22)-\mathrm{C}(72)$ & $4.4(8)$ \\
\hline $\mathrm{C}(12)-\mathrm{C}(22)-\mathrm{C}(32)-\mathrm{C}(42)$ & $2.9(9)$ \\
\hline $\mathrm{C}(72)-\mathrm{C}(22)-\mathrm{C}(32)-\mathrm{C}(42)$ & $-174.9(6)$ \\
\hline $\mathrm{C}(22)-\mathrm{C}(32)-\mathrm{C}(42)-\mathrm{C}(52)$ & $-3.8(10)$ \\
\hline $\mathrm{C}(32)-\mathrm{C}(42)-\mathrm{C}(52)-\mathrm{C}(62)$ & $1.7(10)$ \\
\hline $\mathrm{C}(42)-\mathrm{C}(52)-\mathrm{C}(62)-\mathrm{C}(12)$ & $1.2(10)$ \\
\hline $\mathrm{C}(42)-\mathrm{C}(52)-\mathrm{C}(62)-\mathrm{C}(102)$ & $-178.0(6)$ \\
\hline $\mathrm{N}(11)-\mathrm{C}(12)-\mathrm{C}(62)-\mathrm{C}(52)$ & $171.3(6)$ \\
\hline $\mathrm{C}(22)-\mathrm{C}(12)-\mathrm{C}(62)-\mathrm{C}(52)$ & $-2.1(9)$ \\
\hline $\mathrm{N}(11)-\mathrm{C}(12)-\mathrm{C}(62)-\mathrm{C}(102)$ & $-9.5(9)$ \\
\hline$C(22)-C(12)-C(62)-C(102)$ & $177.1(6)$ \\
\hline $\mathrm{C}(32)-\mathrm{C}(22)-\mathrm{C}(72)-\mathrm{C}(92)$ & $-48.1(8)$ \\
\hline $\mathrm{C}(12)-\mathrm{C}(22)-\mathrm{C}(72)-\mathrm{C}(92)$ & $134.2(6)$ \\
\hline $\mathrm{C}(32)-\mathrm{C}(22)-\mathrm{C}(72)-\mathrm{C}(82)$ & $75.7(7)$ \\
\hline$C(12)-C(22)-C(72)-C(82)$ & $-102.1(7)$ \\
\hline
\end{tabular}




\begin{tabular}{|c|c|}
\hline $\mathrm{C}(52)-\mathrm{C}(62)-\mathrm{C}(102)-\mathrm{C}(112)$ & $-65.9(8)$ \\
\hline$C(12)-C(62)-C(102)-C(112)$ & $114.9(7)$ \\
\hline $\mathrm{C}(52)-\mathrm{C}(62)-\mathrm{C}(102)-\mathrm{C}(122)$ & $57.5(8)$ \\
\hline$C(12)-C(62)-C(102)-C(122)$ & $-121.7(7)$ \\
\hline $\mathrm{C}(41)-\mathrm{N}(21)-\mathrm{C}(13)-\mathrm{C}(63)$ & $-83.6(7)$ \\
\hline $\mathrm{Fe}(1)-\mathrm{N}(21)-\mathrm{C}(13)-\mathrm{C}(63)$ & $98.6(6)$ \\
\hline $\mathrm{C}(41)-\mathrm{N}(21)-\mathrm{C}(13)-\mathrm{C}(23)$ & $99.5(6)$ \\
\hline $\mathrm{Fe}(1)-\mathrm{N}(21)-\mathrm{C}(13)-\mathrm{C}(23)$ & $-78.3(6)$ \\
\hline$C(63)-C(13)-C(23)-C(33)$ & $1.1(9)$ \\
\hline $\mathrm{N}(21)-\mathrm{C}(13)-\mathrm{C}(23)-\mathrm{C}(33)$ & $178.0(5)$ \\
\hline$C(63)-C(13)-C(23)-C(73)$ & $179.3(6)$ \\
\hline $\mathrm{N}(21)-\mathrm{C}(13)-\mathrm{C}(23)-\mathrm{C}(73)$ & $-3.8(9)$ \\
\hline $\mathrm{C}(13)-\mathrm{C}(23)-\mathrm{C}(33)-\mathrm{C}(43)$ & $0.1(10)$ \\
\hline$C(73)-C(23)-C(33)-C(43)$ & $-178.1(7)$ \\
\hline $\mathrm{C}(23)-\mathrm{C}(33)-\mathrm{C}(43)-\mathrm{C}(53)$ & $-0.5(11)$ \\
\hline $\mathrm{C}(33)-\mathrm{C}(43)-\mathrm{C}(53)-\mathrm{C}(63)$ & $-0.2(10)$ \\
\hline $\mathrm{C}(43)-\mathrm{C}(53)-\mathrm{C}(63)-\mathrm{C}(13)$ & $1.3(9)$ \\
\hline $\mathrm{C}(43)-\mathrm{C}(53)-\mathrm{C}(63)-\mathrm{C}(103)$ & $-176.8(6)$ \\
\hline $\mathrm{C}(23)-\mathrm{C}(13)-\mathrm{C}(63)-\mathrm{C}(53)$ & $-1.8(9)$ \\
\hline $\mathrm{N}(21)-\mathrm{C}(13)-\mathrm{C}(63)-\mathrm{C}(53)$ & $-178.7(5)$ \\
\hline $\mathrm{C}(23)-\mathrm{C}(13)-\mathrm{C}(63)-\mathrm{C}(103)$ & $176.3(5)$ \\
\hline $\mathrm{N}(21)-\mathrm{C}(13)-\mathrm{C}(63)-\mathrm{C}(103)$ & $-0.5(9)$ \\
\hline $\mathrm{C}(33)-\mathrm{C}(23)-\mathrm{C}(73)-\mathrm{C}(93)$ & $66.4(9)$ \\
\hline $\mathrm{C}(13)-\mathrm{C}(23)-\mathrm{C}(73)-\mathrm{C}(93)$ & $-111.7(7)$ \\
\hline $\mathrm{C}(33)-\mathrm{C}(23)-\mathrm{C}(73)-\mathrm{C}(83)$ & $-60.0(9)$ \\
\hline $\mathrm{C}(13)-\mathrm{C}(23)-\mathrm{C}(73)-\mathrm{C}(83)$ & $121.9(7)$ \\
\hline $\mathrm{C}(53)-\mathrm{C}(63)-\mathrm{C}(103)-\mathrm{C}(113)$ & $79.5(7)$ \\
\hline $\mathrm{C}(13)-\mathrm{C}(63)-\mathrm{C}(103)-\mathrm{C}(113)$ & $-98.6(7)$ \\
\hline$C(53)-C(63)-C(103)-C(123)$ & $-45.0(8)$ \\
\hline $\mathrm{C}(13)-\mathrm{C}(63)-\mathrm{C}(103)-\mathrm{C}(123)$ & $137.0(7)$ \\
\hline $\mathrm{C}(15)-\mathrm{N}(14)-\mathrm{C}(24)-\mathrm{C}(34)$ & $173.7(6)$ \\
\hline $\mathrm{Fe}(2)-\mathrm{N}(14)-\mathrm{C}(24)-\mathrm{C}(34)$ & $-2.0(8)$ \\
\hline$C(15)-N(14)-C(24)-C(14)$ & $-7.2(8)$ \\
\hline $\mathrm{Fe}(2)-\mathrm{N}(14)-\mathrm{C}(24)-\mathrm{C}(14)$ & $177.0(4)$ \\
\hline $\mathrm{N}(14)-\mathrm{C}(24)-\mathrm{C}(34)-\mathrm{C}(44)$ & $0.5(10)$ \\
\hline$C(14)-C(24)-C(34)-C(44)$ & $-178.6(6)$ \\
\hline
\end{tabular}




\begin{tabular}{|c|c|}
\hline $\mathrm{C}(16)-\mathrm{N}(24)-\mathrm{C}(44)-\mathrm{C}(34)$ & $178.7(5)$ \\
\hline $\mathrm{Fe}(2)-\mathrm{N}(24)-\mathrm{C}(44)-\mathrm{C}(34)$ & $1.1(8)$ \\
\hline $\mathrm{C}(16)-\mathrm{N}(24)-\mathrm{C}(44)-\mathrm{C}(54)$ & $-1.6(8)$ \\
\hline $\mathrm{Fe}(2)-\mathrm{N}(24)-\mathrm{C}(44)-\mathrm{C}(54)$ & $-179.1(5)$ \\
\hline $\mathrm{C}(24)-\mathrm{C}(34)-\mathrm{C}(44)-\mathrm{N}(24)$ & $0.0(10)$ \\
\hline $\mathrm{C}(24)-\mathrm{C}(34)-\mathrm{C}(44)-\mathrm{C}(54)$ & $-179.7(7)$ \\
\hline $\mathrm{C}(24)-\mathrm{N}(14)-\mathrm{C}(15)-\mathrm{C}(65)$ & $108.2(7)$ \\
\hline $\mathrm{Fe}(2)-\mathrm{N}(14)-\mathrm{C}(15)-\mathrm{C}(65)$ & $-75.8(7)$ \\
\hline $\mathrm{C}(24)-\mathrm{N}(14)-\mathrm{C}(15)-\mathrm{C}(25)$ & $-74.8(7)$ \\
\hline $\mathrm{Fe}(2)-\mathrm{N}(14)-\mathrm{C}(15)-\mathrm{C}(25)$ & $101.2(6)$ \\
\hline$C(65)-C(15)-C(25)-C(35)$ & $-4.5(9)$ \\
\hline $\mathrm{N}(14)-\mathrm{C}(15)-\mathrm{C}(25)-\mathrm{C}(35)$ & $178.5(5)$ \\
\hline$C(65)-C(15)-C(25)-C(75)$ & $171.3(6)$ \\
\hline $\mathrm{N}(14)-\mathrm{C}(15)-\mathrm{C}(25)-\mathrm{C}(75)$ & $-5.7(9)$ \\
\hline$C(15)-C(25)-C(35)-C(45)$ & $0.7(10)$ \\
\hline$C(75)-C(25)-C(35)-C(45)$ & $-175.1(6)$ \\
\hline$C(25)-C(35)-C(45)-C(55)$ & $3.9(10)$ \\
\hline $\mathrm{C}(35)-\mathrm{C}(45)-\mathrm{C}(55)-\mathrm{C}(65)$ & $-4.9(10)$ \\
\hline$C(45)-C(55)-C(65)-C(15)$ & $1.3(10)$ \\
\hline $\mathrm{C}(45)-\mathrm{C}(55)-\mathrm{C}(65)-\mathrm{C}(105)$ & $-178.6(7)$ \\
\hline $\mathrm{N}(14)-\mathrm{C}(15)-\mathrm{C}(65)-\mathrm{C}(55)$ & $-179.5(6)$ \\
\hline$C(25)-C(15)-C(65)-C(55)$ & $3.5(10)$ \\
\hline $\mathrm{N}(14)-\mathrm{C}(15)-\mathrm{C}(65)-\mathrm{C}(105)$ & $0.3(10)$ \\
\hline$C(25)-C(15)-C(65)-C(105)$ & $-176.7(6)$ \\
\hline $\mathrm{C}(35)-\mathrm{C}(25)-\mathrm{C}(75)-\mathrm{C}(95)$ & $-39.9(9)$ \\
\hline$C(15)-C(25)-C(75)-C(95)$ & $144.4(7)$ \\
\hline $\mathrm{C}(35)-\mathrm{C}(25)-\mathrm{C}(75)-\mathrm{C}(85)$ & $86.6(8)$ \\
\hline$C(15)-C(25)-C(75)-C(85)$ & $-89.1(7)$ \\
\hline $\mathrm{C}(55)-\mathrm{C}(65)-\mathrm{C}(105)-\mathrm{C}(125)$ & 75.3(9) \\
\hline $\mathrm{C}(15)-\mathrm{C}(65)-\mathrm{C}(105)-\mathrm{C}(125)$ & $-104.5(8)$ \\
\hline $\mathrm{C}(55)-\mathrm{C}(65)-\mathrm{C}(105)-\mathrm{C}(115)$ & $-45.9(10)$ \\
\hline $\mathrm{C}(15)-\mathrm{C}(65)-\mathrm{C}(105)-\mathrm{C}(115)$ & $134.2(8)$ \\
\hline $\mathrm{C}(44)-\mathrm{N}(24)-\mathrm{C}(16)-\mathrm{C}(26)$ & $98.1(7)$ \\
\hline $\mathrm{Fe}(2)-\mathrm{N}(24)-\mathrm{C}(16)-\mathrm{C}(26)$ & $-84.2(6)$ \\
\hline $\mathrm{C}(44)-\mathrm{N}(24)-\mathrm{C}(16)-\mathrm{C}(66)$ & $-84.0(7)$ \\
\hline $\mathrm{Fe}(2)-\mathrm{N}(24)-\mathrm{C}(16)-\mathrm{C}(66)$ & $93.6(6)$ \\
\hline
\end{tabular}




\begin{tabular}{|c|c|}
\hline $\mathrm{N}(24)-\mathrm{C}(16)-\mathrm{C}(26)-\mathrm{C}(36)$ & $176.9(6)$ \\
\hline$C(66)-C(16)-C(26)-C(36)$ & $-1.0(10)$ \\
\hline $\mathrm{N}(24)-\mathrm{C}(16)-\mathrm{C}(26)-\mathrm{C}(76)$ & $-1.9(10)$ \\
\hline$C(66)-C(16)-C(26)-C(76)$ & $-179.8(7)$ \\
\hline$C(16)-C(26)-C(36)-C(46)$ & $2.0(11)$ \\
\hline$C(76)-C(26)-C(36)-C(46)$ & $-179.2(8)$ \\
\hline$C(26)-C(36)-C(46)-C(56)$ & $-1.4(11)$ \\
\hline$C(36)-C(46)-C(56)-C(66)$ & $-0.3(10)$ \\
\hline$C(46)-C(56)-C(66)-C(16)$ & $1.2(9)$ \\
\hline$C(46)-C(56)-C(66)-C(106)$ & $-176.9(6)$ \\
\hline$C(26)-C(16)-C(66)-C(56)$ & $-0.6(9)$ \\
\hline $\mathrm{N}(24)-\mathrm{C}(16)-\mathrm{C}(66)-\mathrm{C}(56)$ & $-178.5(5)$ \\
\hline$C(26)-C(16)-C(66)-C(106)$ & $177.5(6)$ \\
\hline $\mathrm{N}(24)-\mathrm{C}(16)-\mathrm{C}(66)-\mathrm{C}(106)$ & $-0.4(8)$ \\
\hline$C(36)-C(26)-C(76)-C(86)$ & $55.6(11)$ \\
\hline$C(16)-C(26)-C(76)-C(86)$ & $-125.7(8)$ \\
\hline$C(36)-C(26)-C(76)-C(96)$ & $-69.5(13)$ \\
\hline$C(16)-C(26)-C(76)-C(96)$ & $109.3(11)$ \\
\hline$C(56)-C(66)-C(106)-C(116)$ & $83.4(7)$ \\
\hline$C(16)-C(66)-C(106)-C(116)$ & $-94.6(7)$ \\
\hline$C(56)-C(66)-C(106)-C(126)$ & $-39.9(8)$ \\
\hline$C(16)-C(66)-C(106)-C(126)$ & $142.0(6)$ \\
\hline $\mathrm{C}(120)-\mathrm{O}(10)-\mathrm{C}(10)-\mathrm{C}(20)$ & $175.4(8)$ \\
\hline $\mathrm{K}(1)-\mathrm{O}(10)-\mathrm{C}(10)-\mathrm{C}(20)$ & $32.3(10)$ \\
\hline $\mathrm{C}(30)-\mathrm{O}(20)-\mathrm{C}(20)-\mathrm{C}(10)$ & $-172.8(8)$ \\
\hline $\mathrm{K}(1)-\mathrm{O}(20)-\mathrm{C}(20)-\mathrm{C}(10)$ & $63.2(9)$ \\
\hline $\mathrm{O}(10)-\mathrm{C}(10)-\mathrm{C}(20)-\mathrm{O}(20)$ & $-65.6(11)$ \\
\hline $\mathrm{C}(20)-\mathrm{O}(20)-\mathrm{C}(30)-\mathrm{C}(40)$ & $176.0(8)$ \\
\hline $\mathrm{K}(1)-\mathrm{O}(20)-\mathrm{C}(30)-\mathrm{C}(40)$ & $-61.6(8)$ \\
\hline $\mathrm{C}(50)-\mathrm{O}(30)-\mathrm{C}(40)-\mathrm{C}(30)$ & $-173.6(7)$ \\
\hline $\mathrm{K}(1)-\mathrm{O}(30)-\mathrm{C}(40)-\mathrm{C}(30)$ & $-32.1(8)$ \\
\hline $\mathrm{O}(20)-\mathrm{C}(30)-\mathrm{C}(40)-\mathrm{O}(30)$ & $63.6(10)$ \\
\hline $\mathrm{C}(40)-\mathrm{O}(30)-\mathrm{C}(50)-\mathrm{C}(60)$ & $-177.2(7)$ \\
\hline $\mathrm{K}(1)-\mathrm{O}(30)-\mathrm{C}(50)-\mathrm{C}(60)$ & $39.7(8)$ \\
\hline $\mathrm{C}(70)-\mathrm{O}(40)-\mathrm{C}(60)-\mathrm{C}(50)$ & $-83.8(9)$ \\
\hline $\mathrm{K}(1)-\mathrm{O}(40)-\mathrm{C}(60)-\mathrm{C}(50)$ & $53.7(8)$ \\
\hline
\end{tabular}




\begin{tabular}{|c|c|}
\hline $\mathrm{O}(30)-\mathrm{C}(50)-\mathrm{C}(60)-\mathrm{O}(40)$ & $-63.6(9)$ \\
\hline $\mathrm{C}(60)-\mathrm{O}(40)-\mathrm{C}(70)-\mathrm{C}(80)$ & $-171.1(7)$ \\
\hline $\mathrm{K}(1)-\mathrm{O}(40)-\mathrm{C}(70)-\mathrm{C}(80)$ & $53.5(8)$ \\
\hline $\mathrm{C}(90)-\mathrm{O}(50)-\mathrm{C}(80)-\mathrm{C}(70)$ & $177.7(7)$ \\
\hline $\mathrm{K}(1)-\mathrm{O}(50)-\mathrm{C}(80)-\mathrm{C}(70)$ & $49.4(8)$ \\
\hline $\mathrm{O}(40)-\mathrm{C}(70)-\mathrm{C}(80)-\mathrm{O}(50)$ & $-68.7(9)$ \\
\hline $\mathrm{C}(80)-\mathrm{O}(50)-\mathrm{C}(90)-\mathrm{C}(100)$ & $179.9(7)$ \\
\hline $\mathrm{K}(1)-\mathrm{O}(50)-\mathrm{C}(90)-\mathrm{C}(100)$ & $-52.1(8)$ \\
\hline$C(110)-O(60)-C(100)-C(90)$ & $177.9(8)$ \\
\hline $\mathrm{K}(1)-\mathrm{O}(60)-\mathrm{C}(100)-\mathrm{C}(90)$ & $-46.8(9)$ \\
\hline $\mathrm{O}(50)-\mathrm{C}(90)-\mathrm{C}(100)-\mathrm{O}(60)$ & $67.7(9)$ \\
\hline $\mathrm{C}(100)-\mathrm{O}(60)-\mathrm{C}(110)-\mathrm{C}(120)$ & $85.8(9)$ \\
\hline $\mathrm{K}(1)-\mathrm{O}(60)-\mathrm{C}(110)-\mathrm{C}(120)$ & $-51.0(9)$ \\
\hline$C(10)-O(10)-C(120)-C(110)$ & $173.7(8)$ \\
\hline $\mathrm{K}(1)-\mathrm{O}(10)-\mathrm{C}(120)-\mathrm{C}(110)$ & $-41.3(9)$ \\
\hline $\mathrm{O}(60)-\mathrm{C}(110)-\mathrm{C}(120)-\mathrm{O}(10)$ & $63.0(11)$ \\
\hline $\mathrm{C}(200)-\mathrm{O}(70)-\mathrm{C}(130)-\mathrm{C}(140)$ & $-82.2(8)$ \\
\hline $\mathrm{K}(1)-\mathrm{O}(70)-\mathrm{C}(130)-\mathrm{C}(140)$ & $67.1(7)$ \\
\hline $\mathrm{C}(200)-\mathrm{O}(70)-\mathrm{C}(130)-\mathrm{K}(1)$ & $-149.3(7)$ \\
\hline $\mathrm{C}(150)-\mathrm{O}(80)-\mathrm{C}(140)-\mathrm{C}(130)$ & $153.9(7)$ \\
\hline $\mathrm{K}(1)-\mathrm{O}(80)-\mathrm{C}(140)-\mathrm{C}(130)$ & $22.8(9)$ \\
\hline $\mathrm{O}(70)-\mathrm{C}(130)-\mathrm{C}(140)-\mathrm{O}(80)$ & $-60.3(9)$ \\
\hline $\mathrm{K}(1)-\mathrm{C}(130)-\mathrm{C}(140)-\mathrm{O}(80)$ & $-16.5(6)$ \\
\hline $\mathrm{C}(140)-\mathrm{O}(80)-\mathrm{C}(150)-\mathrm{C}(160)$ & $-77.7(9)$ \\
\hline $\mathrm{K}(1)-\mathrm{O}(80)-\mathrm{C}(150)-\mathrm{C}(160)$ & $57.0(8)$ \\
\hline $\mathrm{C}(170)-\mathrm{O}(90)-\mathrm{C}(160)-\mathrm{C}(150)$ & $170.3(7)$ \\
\hline $\mathrm{K}(1)-\mathrm{O}(90)-\mathrm{C}(160)-\mathrm{C}(150)$ & $32.0(9)$ \\
\hline $\mathrm{O}(80)-\mathrm{C}(150)-\mathrm{C}(160)-\mathrm{O}(90)$ & $-60.5(10)$ \\
\hline $\mathrm{C}(160)-\mathrm{O}(90)-\mathrm{C}(170)-\mathrm{C}(180)$ & $-76.0(9)$ \\
\hline $\mathrm{K}(1)-\mathrm{O}(90)-\mathrm{C}(170)-\mathrm{C}(180)$ & $66.0(8)$ \\
\hline $\mathrm{C}(190)-\mathrm{O}(100)-\mathrm{C}(180)-\mathrm{C}(170)$ & $155.0(7)$ \\
\hline $\mathrm{K}(1)-\mathrm{O}(100)-\mathrm{C}(180)-\mathrm{C}(170)$ & $28.2(9)$ \\
\hline $\mathrm{O}(90)-\mathrm{C}(170)-\mathrm{C}(180)-\mathrm{O}(100)$ & $-63.5(10)$ \\
\hline $\mathrm{C}(180)-\mathrm{O}(100)-\mathrm{C}(190)-\mathrm{C}(200)$ & $-77.4(9)$ \\
\hline $\mathrm{K}(1)-\mathrm{O}(100)-\mathrm{C}(190)-\mathrm{C}(200)$ & $53.3(8)$ \\
\hline$C(130)-O(70)-C(200)-C(190)$ & $173.6(7)$ \\
\hline
\end{tabular}




\begin{tabular}{|c|c|}
\hline $\mathrm{K}(1)-\mathrm{O}(70)-\mathrm{C}(200)-\mathrm{C}(190)$ & $31.6(9)$ \\
\hline $\mathrm{O}(100)-\mathrm{C}(190)-\mathrm{C}(200)-\mathrm{O}(70)$ & $-57.1(10)$ \\
\hline $\mathrm{C}(210) \# 1-\mathrm{O}(110)-\mathrm{C}(210)-\mathrm{C}(220)$ & $-179.8(5)$ \\
\hline $\mathrm{K}(2)-\mathrm{O}(110)-\mathrm{C}(210)-\mathrm{C}(220)$ & $-46.7(9)$ \\
\hline $\mathrm{C}(230)-\mathrm{O}(120)-\mathrm{C}(220)-\mathrm{C}(210)$ & $172.3(7)$ \\
\hline $\mathrm{K}(2)-\mathrm{O}(120)-\mathrm{C}(220)-\mathrm{C}(210)$ & $-50.5(9)$ \\
\hline $\mathrm{O}(110)-\mathrm{C}(210)-\mathrm{C}(220)-\mathrm{O}(120)$ & $65.0(10)$ \\
\hline $\mathrm{C}(220)-\mathrm{O}(120)-\mathrm{C}(230)-\mathrm{C}(240)$ & $79.8(9)$ \\
\hline $\mathrm{K}(2)-\mathrm{O}(120)-\mathrm{C}(230)-\mathrm{C}(240)$ & $-58.5(7)$ \\
\hline $\mathrm{C}(250)-\mathrm{O}(130)-\mathrm{C}(240)-\mathrm{C}(230)$ & $-177.9(6)$ \\
\hline $\mathrm{K}(2)-\mathrm{O}(130)-\mathrm{C}(240)-\mathrm{C}(230)$ & $-34.8(8)$ \\
\hline $\mathrm{O}(120)-\mathrm{C}(230)-\mathrm{C}(240)-\mathrm{O}(130)$ & $61.5(9)$ \\
\hline $\mathrm{C}(240)-\mathrm{O}(130)-\mathrm{C}(250)-\mathrm{C}(260)$ & $178.2(6)$ \\
\hline $\mathrm{K}(2)-\mathrm{O}(130)-\mathrm{C}(250)-\mathrm{C}(260)$ & $35.5(7)$ \\
\hline $\mathrm{C}(260) \# 1-\mathrm{O}(140)-\mathrm{C}(260)-\mathrm{C}(250)$ & $-172.9(4)$ \\
\hline $\mathrm{K}(2)-\mathrm{O}(140)-\mathrm{C}(260)-\mathrm{C}(250)$ & $63.2(7)$ \\
\hline $\mathrm{O}(130)-\mathrm{C}(250)-\mathrm{C}(260)-\mathrm{O}(140)$ & $-66.0(8)$ \\
\hline $\mathrm{C}(8 \mathrm{X}) \# 1-\mathrm{O}(150)-\mathrm{C}(8 \mathrm{X})-\mathrm{C}(7 \mathrm{X})$ & $-143.1(10)$ \\
\hline $\mathrm{K}(2)-\mathrm{O}(150)-\mathrm{C}(8 \mathrm{X})-\mathrm{C}(7 \mathrm{X})$ & $0(3)$ \\
\hline $\mathrm{C}(6 \mathrm{Y})-\mathrm{O}(160)-\mathrm{C}(7 \mathrm{Y})-\mathrm{C}(8 \mathrm{Y})$ & $162.0(15)$ \\
\hline $\mathrm{K}(2)-\mathrm{O}(160)-\mathrm{C}(7 \mathrm{Y})-\mathrm{C}(8 \mathrm{Y})$ & $22.3(19)$ \\
\hline $\mathrm{C}(7 \mathrm{X})-\mathrm{O}(160)-\mathrm{C}(6 \mathrm{X})-\mathrm{C}(5 \mathrm{X})$ & $-164.2(14)$ \\
\hline $\mathrm{K}(2)-\mathrm{O}(160)-\mathrm{C}(6 \mathrm{X})-\mathrm{C}(5 \mathrm{X})$ & $-31.3(17)$ \\
\hline $\mathrm{C}(6 \mathrm{Y})-\mathrm{C}(5 \mathrm{Y})-\mathrm{O}(170)-\mathrm{C}(5 \mathrm{Y}) \# 1$ & $145.0(11)$ \\
\hline $\mathrm{C}(6 \mathrm{Y})-\mathrm{C}(5 \mathrm{Y})-\mathrm{O}(170)-\mathrm{K}(2)$ & $8(2)$ \\
\hline $\mathrm{C}(310) \# 1-\mathrm{O}(180)-\mathrm{C}(310)-\mathrm{C}(320)$ & $-123.0(17)$ \\
\hline $\mathrm{K}(3)-\mathrm{O}(180)-\mathrm{C}(310)-\mathrm{C}(320)$ & $33(2)$ \\
\hline $\mathrm{O}(180)-\mathrm{C}(310)-\mathrm{C}(320)-\mathrm{O}(190)$ & $-29(3)$ \\
\hline $\mathrm{C}(330)-\mathrm{O}(190)-\mathrm{C}(320)-\mathrm{C}(310)$ & $-171.8(16)$ \\
\hline $\mathrm{K}(3)-\mathrm{O}(190)-\mathrm{C}(320)-\mathrm{C}(310)$ & $6(2)$ \\
\hline $\mathrm{C}(320)-\mathrm{O}(190)-\mathrm{C}(330)-\mathrm{C}(340)$ & $179.9(15)$ \\
\hline $\mathrm{K}(3)-\mathrm{O}(190)-\mathrm{C}(330)-\mathrm{C}(340)$ & $1.8(17)$ \\
\hline $\mathrm{C}(380)-\mathrm{O}(220)-\mathrm{C}(370)-\mathrm{C}(370) \# 1$ & $-124.5(8)$ \\
\hline $\mathrm{K}(3)-\mathrm{O}(220)-\mathrm{C}(370)-\mathrm{C}(370) \# 1$ & $13.0(9)$ \\
\hline $\mathrm{C}(370)-\mathrm{O}(220)-\mathrm{C}(380)-\mathrm{C}(390)$ & 119.9(16) \\
\hline $\mathrm{K}(3)-\mathrm{O}(220)-\mathrm{C}(380)-\mathrm{C}(390)$ & $-18(2)$ \\
\hline
\end{tabular}




\begin{tabular}{lc}
$\mathrm{O}(220)-\mathrm{C}(380)-\mathrm{C}(390)-\mathrm{O}(230)$ & $0(3)$ \\
$\mathrm{C}(400)-\mathrm{O}(230)-\mathrm{C}(390)-\mathrm{C}(380)$ & $-122.8(16)$ \\
$\mathrm{K}(3)-\mathrm{O}(230)-\mathrm{C}(390)-\mathrm{C}(380)$ & $18.3(19)$ \\
$\mathrm{C}(2 \mathrm{X})-\mathrm{O}(1 \mathrm{X})-\mathrm{C}(3 \mathrm{X})-\mathrm{C}(4 \mathrm{X})$ & $178.5(9)$ \\
$\mathrm{C}(3 \mathrm{X})-\mathrm{O}(1 \mathrm{X})-\mathrm{C}(2 \mathrm{X})-\mathrm{C}(1 \mathrm{X})$ & $-176.1(9)$ \\
$\mathrm{C}(390)-\mathrm{O}(230)-\mathrm{C}(400)-\mathrm{C}(400) \# 1$ & $124.0(8)$ \\
$\mathrm{K}(3)-\mathrm{O}(230)-\mathrm{C}(400)-\mathrm{C}(400) \# 1$ & $-17.8(9)$ \\
$\mathrm{C}(350)-\mathrm{C}(360)-\mathrm{O}(210)-\mathrm{C}(360) \# 1$ & $173.3(10)$ \\
$\mathrm{C}(350)-\mathrm{C}(360)-\mathrm{O}(210)-\mathrm{K}(3)$ & $34(2)$ \\
$\mathrm{C}(350)-\mathrm{O}(200)-\mathrm{C}(340)-\mathrm{C}(330)$ & $172.4(18)$ \\
$\mathrm{K}(3)-\mathrm{O}(200)-\mathrm{C}(340)-\mathrm{C}(330)$ & $-2(3)$ \\
$\mathrm{O}(190)-\mathrm{C}(330)-\mathrm{C}(340)-\mathrm{O}(200)$ & $0(3)$ \\
$\mathrm{O}(210)-\mathrm{C}(360)-\mathrm{C}(350)-\mathrm{O}(200)$ & $-54(3)$ \\
$\mathrm{C}(340)-\mathrm{O}(200)-\mathrm{C}(350)-\mathrm{C}(360)$ & $-127(2)$ \\
$\mathrm{K}(3)-\mathrm{O}(200)-\mathrm{C}(350)-\mathrm{C}(360)$ & $47(2)$ \\
$\mathrm{O}(160)-\mathrm{C}(6 \mathrm{X})-\mathrm{C}(5 \mathrm{X})-\mathrm{O}(170)$ & $63(3)$ \\
$\mathrm{C}(5 \mathrm{X}) \# 1-\mathrm{O}(170)-\mathrm{C}(5 \mathrm{X})-\mathrm{C}(6 \mathrm{X})$ & $62(4)$ \\
$\mathrm{K}(2)-\mathrm{O}(170)-\mathrm{C}(5 \mathrm{X})-\mathrm{C}(6 \mathrm{X})$ & $-60(3)$ \\
$\mathrm{O}(170)-\mathrm{C}(5 \mathrm{Y})-\mathrm{C}(6 \mathrm{Y})-\mathrm{O}(160)$ & $-45(3)$ \\
$\mathrm{C}(7 \mathrm{Y})-\mathrm{O}(160)-\mathrm{C}(6 \mathrm{Y})-\mathrm{C}(5 \mathrm{Y})$ & $-89(2)$ \\
$\mathrm{K}(2)-\mathrm{O}(160)-\mathrm{C}(6 \mathrm{Y})-\mathrm{C}(5 \mathrm{Y})$ & $59(2)$ \\
$\mathrm{O}(150)-\mathrm{C}(8 \mathrm{X})-\mathrm{C}(7 \mathrm{X})-\mathrm{O}(160)$ & $39(3)$ \\
$\mathrm{C}(6 \mathrm{X})-\mathrm{O}(160)-\mathrm{C}(7 \mathrm{X})-\mathrm{C}(8 \mathrm{X})$ & $87(2)$ \\
$\mathrm{K}(2)-\mathrm{O}(160)-\mathrm{C}(7 \mathrm{X})-\mathrm{C}(8 \mathrm{X})$ & $-56(2)$ \\
$\mathrm{O}(160)-\mathrm{C}(7 \mathrm{Y})-\mathrm{C}(8 \mathrm{Y})-\mathrm{O}(150)$ & $-56(3)$ \\
$\mathrm{C}(8 \mathrm{Y}) \# 1-\mathrm{O}(150)-\mathrm{C}(8 \mathrm{Y})-\mathrm{C}(7 \mathrm{Y})$ & $-67(4)$ \\
$\mathrm{K}(2)-\mathrm{O}(150)-\mathrm{C}(8 \mathrm{Y})-\mathrm{C}(7 \mathrm{Y})$ & \\
\hline
\end{tabular}

Symmetry transformations used to generate equivalent atoms:

$\# 1 \mathrm{x},-\mathrm{y}+1 / 2, \mathrm{z}$ 
$\mathrm{L}^{\mathrm{Me}} \mathbf{F e}\left(\mathrm{OCPh}_{2} \mathbf{C H C P h}\right)$ (6). Low-temperature diffraction data ( $\omega$-scans) were collected on a Rigaku MicroMax-007HF diffractometer coupled to a Saturn994+ CCD detector with $\mathrm{Cu} \mathrm{K} \alpha(\lambda=$ $1.54178 \AA$ ) for the structure of 6 . The diffraction images were processed and scaled using Rigaku Oxford Diffraction software (CrysAlisPro; Rigaku OD: The Woodlands, TX, 2015). The structure was solved with SHELXT and was refined against $F^{2}$ on all data by full-matrix least squares with SHELXL (Sheldrick, G. M. Acta Cryst. 2008, A64, 112-122). All non-hydrogen atoms were refined anisotropically. Hydrogen atoms were included in the model at geometrically calculated positions and refined using a riding model. The isotropic displacement parameters of all hydrogen atoms were fixed to 1.2 times the $\mathrm{U}$ value of the atoms to which they are linked (1.5 times for methyl groups. 


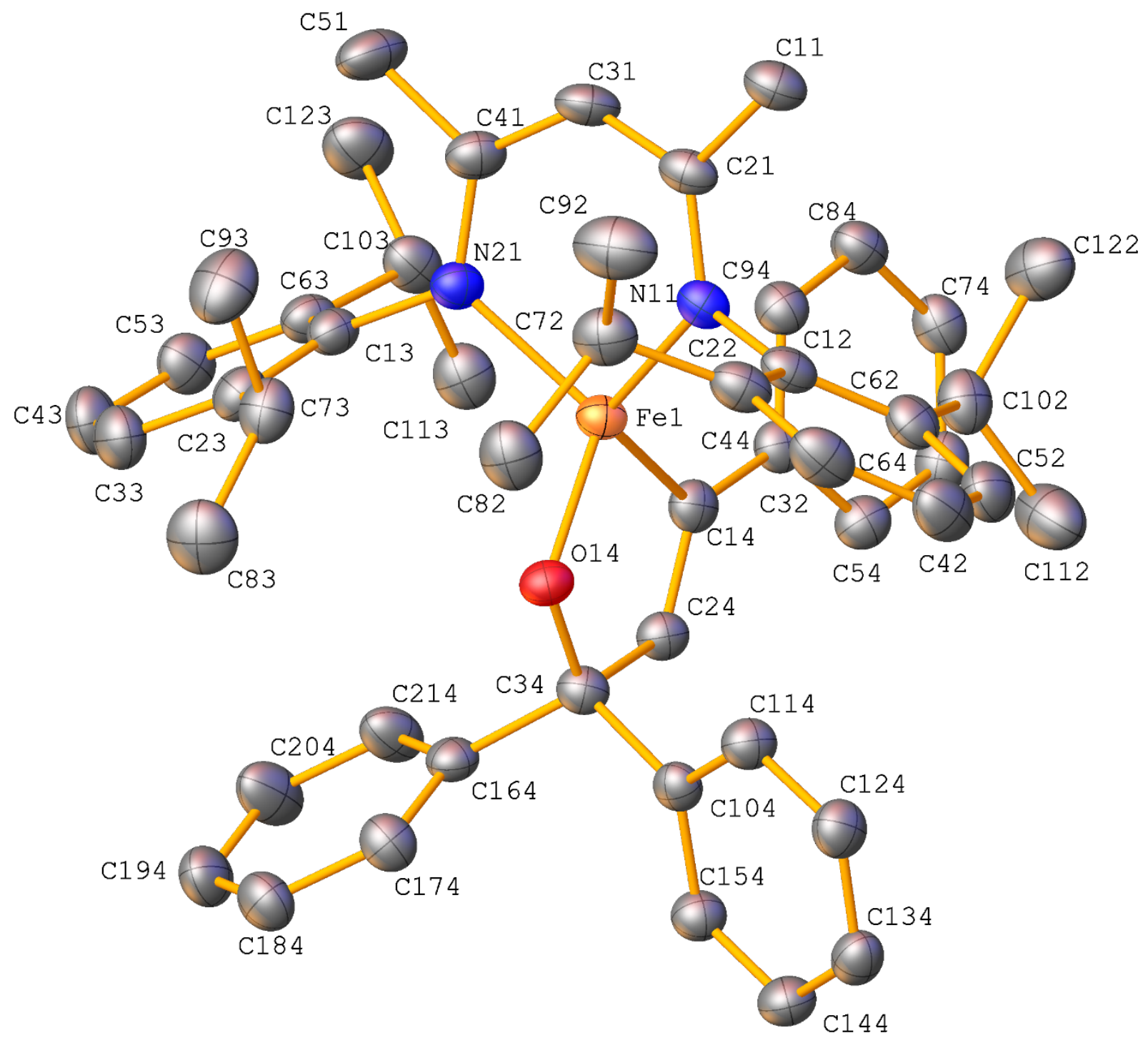

Figure S-28: The complete numbering scheme of $\mathbf{L}^{\mathrm{Me}} \mathbf{F e}\left(\mathbf{O C P h} \mathbf{C H C H}_{2} \mathbf{C H C}\right)(6)$ with $50 \%$ thermal ellipsoid probability levels. The hydrogen atoms are omitted for clarity. 
Table S-24. Crystal data and structure refinement for 6.

Identification code

CSD Deposition Number

Empirical formula

Formula weight

Temperature

Wavelength

Crystal system

Space group

Unit cell dimensions

Volume

Z

Density (calculated)

Absorption coefficient

$\mathrm{F}(000)$

Crystal size

Crystal color and habit

Diffractometer

Theta range for data collection

Index ranges

Reflections collected

Independent reflections

Observed reflections (I > 2sigma(I))

Completeness to theta $=66.600^{\circ}$

Absorption correction

Max. and min. transmission

Solution method

Refinement method

Data / restraints / parameters

Goodness-of-fit on $\mathrm{F}^{2}$

Final $R$ indices [I $>2 \operatorname{sigma}(\mathrm{I})]$

$\mathrm{R}$ indices (all data)

Absolute structure parameter

Largest diff. peak and hole 007b-18035

1944812

C50 H57 Fe N2 O

757.82

93(2) K

\section{$1.54184 \AA$}

Monoclinic

P21

$a=13.1153(3) \AA$

$\alpha=90^{\circ}$.

$b=11.5562(3) \AA$ $\beta=97.040(2)^{\circ}$.

$c=14.0967(4) \AA$ $\gamma=90^{\circ}$.

2120.43(10) $\AA^{3}$

2

$1.187 \mathrm{~g} / \mathrm{cm}^{3}$

$3.130 \mathrm{~mm}^{-1}$

810

$0.200 \times 0.200 \times 0.030 \mathrm{~mm}^{3}$

Green Plate

Rigaku Saturn 944+ CCD

3.159 to $66.600^{\circ}$.

$-15<=\mathrm{h}<=15,-13<=\mathrm{k}<=13,-16<=\mathrm{l}<=16$

76162

$7495[\mathrm{R}($ int $)=0.0861]$

6924

$100.0 \%$

Semi-empirical from equivalents

1.00000 and 0.86231

SHELXT-2014/5 (Sheldrick, 2014)

SHELXL-2014/7 (Sheldrick, 2014)

7495 / 1 / 497

1.027

$\mathrm{R} 1=0.0344, \mathrm{wR} 2=0.0799$

$\mathrm{R} 1=0.0396, \mathrm{wR} 2=0.0827$

$-0.007(2)$

0.353 and -0.252 e. $\AA^{-3}$ 
Table S-25. Atomic coordinates ( $\times 10^{4}$ ) and equivalent isotropic displacement parameters $\left(\AA^{2} \times 10^{3}\right)$ for 6. $U(e q)$ is defined as one third of the trace of the orthogonalized $U^{i j}$ tensor.

\begin{tabular}{|c|c|c|c|c|}
\hline & $\mathrm{x}$ & $\mathrm{y}$ & $\mathrm{z}$ & $\mathrm{U}(\mathrm{eq})$ \\
\hline $\mathrm{Fe}(01)$ & $7500(1)$ & $5547(1)$ & 7097(1) & $24(1)$ \\
\hline $\mathrm{N}(11)$ & $8237(2)$ & $7054(2)$ & $7193(2)$ & $26(1)$ \\
\hline $\mathrm{N}(21)$ & $6160(2)$ & $6169(2)$ & $7384(2)$ & $27(1)$ \\
\hline $\mathrm{C}(11)$ & $8287(3)$ & $9187(3)$ & 7072(3) & $34(1)$ \\
\hline$C(21)$ & 7724(3) & $8054(3)$ & $7096(2)$ & $27(1)$ \\
\hline$C(31)$ & $6649(3)$ & $8129(3)$ & $7065(3)$ & $33(1)$ \\
\hline$C(41)$ & $5937(3)$ & 7298(3) & $7267(3)$ & $31(1)$ \\
\hline$C(51)$ & $4869(3)$ & $7727(4)$ & 7371(3) & $45(1)$ \\
\hline$C(12)$ & $9344(2)$ & $7049(3)$ & $7425(2)$ & $27(1)$ \\
\hline$C(22)$ & $9763(3)$ & $7273(3)$ & $8377(2)$ & $32(1)$ \\
\hline$C(32)$ & 10831(3) & $7195(3)$ & $8597(3)$ & $38(1)$ \\
\hline$C(42)$ & $11455(3)$ & 6894(4) & 7921(3) & $41(1)$ \\
\hline$C(52)$ & $11025(3)$ & $6675(3)$ & 6990(3) & $39(1)$ \\
\hline $\mathrm{C}(62)$ & 9968(3) & $6746(3)$ & $6724(2)$ & $31(1)$ \\
\hline$C(72)$ & 9104(3) & $7552(3)$ & $9159(2)$ & $36(1)$ \\
\hline$C(82)$ & $9127(3)$ & $6535(4)$ & 9861(3) & $42(1)$ \\
\hline $\mathrm{C}(92)$ & 9437(4) & $8684(4)$ & $9676(3)$ & $51(1)$ \\
\hline$C(102)$ & $9514(3)$ & $6518(3)$ & $5694(3)$ & $36(1)$ \\
\hline$C(112)$ & 10111(3) & $5628(4)$ & $5195(3)$ & $52(1)$ \\
\hline$C(122)$ & 9398(3) & 7641(4) & $5118(3)$ & $45(1)$ \\
\hline$C(13)$ & $5411(2)$ & $5378(3)$ & $7684(2)$ & $27(1)$ \\
\hline$C(23)$ & $5528(3)$ & $5005(3)$ & $8638(2)$ & $31(1)$ \\
\hline$C(33)$ & $4806(3)$ & $4250(3)$ & $8932(3)$ & $38(1)$ \\
\hline$C(43)$ & 3983(3) & $3867(4)$ & $8300(3)$ & $40(1)$ \\
\hline$C(53)$ & $3897(3)$ & $4203(3)$ & $7356(3)$ & $36(1)$ \\
\hline$C(63)$ & $4604(2)$ & 4955(3) & 7021(2) & $30(1)$ \\
\hline$C(73)$ & $6417(2)$ & $5442(4)$ & $9357(2)$ & $35(1)$ \\
\hline$C(83)$ & 6784(3) & $4545(4)$ & $10128(3)$ & $49(1)$ \\
\hline$C(93)$ & $6143(3)$ & $6586(4)$ & $9810(3)$ & $51(1)$ \\
\hline$C(103)$ & $4504(3)$ & $5240(3)$ & $5957(2)$ & $34(1)$ \\
\hline$C(113)$ & $4724(3)$ & $4167(4)$ & $5379(3)$ & $40(1)$ \\
\hline$C(123)$ & $3436(3)$ & $5736(4)$ & $5580(3)$ & $47(1)$ \\
\hline
\end{tabular}




\begin{tabular}{lrrrl}
$\mathrm{O}(14)$ & $8047(2)$ & $4367(2)$ & $7879(2)$ & $26(1)$ \\
$\mathrm{C}(14)$ & $7541(2)$ & $4500(3)$ & $5945(2)$ & $27(1)$ \\
$\mathrm{C}(24)$ & $7868(2)$ & $3476(3)$ & $6310(2)$ & $26(1)$ \\
$\mathrm{C}(34)$ & $8193(2)$ & $3315(3)$ & $7388(2)$ & $26(1)$ \\
$\mathrm{C}(44)$ & $7293(2)$ & $4762(3)$ & $4915(2)$ & $26(1)$ \\
$\mathrm{C}(54)$ & $7595(3)$ & $4057(3)$ & $4187(2)$ & $31(1)$ \\
$\mathrm{C}(64)$ & $7355(3)$ & $4342(3)$ & $3233(2)$ & $33(1)$ \\
$\mathrm{C}(74)$ & $6804(2)$ & $5342(3)$ & $2974(2)$ & $32(1)$ \\
$\mathrm{C}(84)$ & $6515(3)$ & $6064(3)$ & $3677(3)$ & $33(1)$ \\
$\mathrm{C}(94)$ & $6768(2)$ & $5780(3)$ & $4630(2)$ & $30(1)$ \\
$\mathrm{C}(104)$ & $9345(2)$ & $3008(3)$ & $7494(2)$ & $26(1)$ \\
$\mathrm{C}(114)$ & $10086(3)$ & $3860(3)$ & $7732(2)$ & $30(1)$ \\
$\mathrm{C}(124)$ & $11123(3)$ & $3592(3)$ & $7779(3)$ & $34(1)$ \\
$\mathrm{C}(134)$ & $11433(3)$ & $2474(3)$ & $7615(2)$ & $32(1)$ \\
$\mathrm{C}(144)$ & $10705(3)$ & $1624(3)$ & $7384(2)$ & $33(1)$ \\
$\mathrm{C}(154)$ & $9670(3)$ & $1893(3)$ & $7316(2)$ & $30(1)$ \\
$\mathrm{C}(164)$ & $7561(2)$ & $2385(3)$ & $7832(2)$ & $27(1)$ \\
$\mathrm{C}(174)$ & $7925(3)$ & $1927(3)$ & $8715(2)$ & $32(1)$ \\
$\mathrm{C}(184)$ & $7352(3)$ & $1135(3)$ & $9171(3)$ & $37(1)$ \\
$\mathrm{C}(194)$ & $6395(3)$ & $790(3)$ & $8738(3)$ & $40(1)$ \\
$\mathrm{C}(204)$ & $6029(3)$ & $1246(4)$ & $7858(3)$ & $46(1)$ \\
$\mathrm{C}(214)$ & $6601(3)$ & $2043(3)$ & $7408(3)$ & $39(1)$ \\
\hline
\end{tabular}


Table S-26. Bond lengths $[\AA]$ and angles $\left[{ }^{\circ}\right]$ for $\mathbf{6}$.

\begin{tabular}{|c|c|}
\hline $\mathrm{Fe}(01)-\mathrm{O}(14)$ & $1.843(2)$ \\
\hline $\mathrm{Fe}(01)-\mathrm{N}(21)$ & $1.986(3)$ \\
\hline $\mathrm{Fe}(01)-\mathrm{N}(11)$ & $1.988(3)$ \\
\hline $\mathrm{Fe}(01)-\mathrm{C}(14)$ & $2.031(3)$ \\
\hline $\mathrm{N}(11)-\mathrm{C}(21)$ & $1.336(4)$ \\
\hline $\mathrm{N}(11)-\mathrm{C}(12)$ & $1.449(4)$ \\
\hline $\mathrm{N}(21)-\mathrm{C}(41)$ & $1.342(4)$ \\
\hline $\mathrm{N}(21)-\mathrm{C}(13)$ & $1.443(4)$ \\
\hline$C(11)-C(21)$ & $1.505(5)$ \\
\hline $\mathrm{C}(11)-\mathrm{H}(11 \mathrm{~A})$ & 0.9800 \\
\hline $\mathrm{C}(11)-\mathrm{H}(11 \mathrm{~B})$ & 0.9800 \\
\hline $\mathrm{C}(11)-\mathrm{H}(11 \mathrm{C})$ & 0.9800 \\
\hline $\mathrm{C}(21)-\mathrm{C}(31)$ & $1.408(5)$ \\
\hline$C(31)-C(41)$ & $1.393(5)$ \\
\hline $\mathrm{C}(31)-\mathrm{H}(31)$ & 0.9500 \\
\hline$C(41)-C(51)$ & $1.509(5)$ \\
\hline $\mathrm{C}(51)-\mathrm{H}(51 \mathrm{~A})$ & 0.9800 \\
\hline $\mathrm{C}(51)-\mathrm{H}(51 \mathrm{~B})$ & 0.9800 \\
\hline $\mathrm{C}(51)-\mathrm{H}(51 \mathrm{C})$ & 0.9800 \\
\hline$C(12)-C(62)$ & $1.402(5)$ \\
\hline$C(12)-C(22)$ & $1.410(5)$ \\
\hline$C(22)-C(32)$ & $1.400(5)$ \\
\hline$C(22)-C(72)$ & $1.516(5)$ \\
\hline$C(32)-C(42)$ & $1.375(6)$ \\
\hline $\mathrm{C}(32)-\mathrm{H}(32)$ & 0.9500 \\
\hline$C(42)-C(52)$ & $1.386(6)$ \\
\hline $\mathrm{C}(42)-\mathrm{H}(42)$ & 0.9500 \\
\hline$C(52)-C(62)$ & $1.393(5)$ \\
\hline $\mathrm{C}(52)-\mathrm{H}(52)$ & 0.9500 \\
\hline $\mathrm{C}(62)-\mathrm{C}(102)$ & $1.523(5)$ \\
\hline $\mathrm{C}(72)-\mathrm{C}(82)$ & $1.534(5)$ \\
\hline $\mathrm{C}(72)-\mathrm{C}(92)$ & $1.535(6)$ \\
\hline $\mathrm{C}(72)-\mathrm{H}(72)$ & 1.0000 \\
\hline $\mathrm{C}(82)-\mathrm{H}(82 \mathrm{~A})$ & 0.9800 \\
\hline $\mathrm{C}(82)-\mathrm{H}(82 \mathrm{~B})$ & 0.9800 \\
\hline
\end{tabular}




\begin{tabular}{|c|c|}
\hline $\mathrm{C}(82)-\mathrm{H}(82 \mathrm{C})$ & 0.9800 \\
\hline $\mathrm{C}(92)-\mathrm{H}(92 \mathrm{~A})$ & 0.9800 \\
\hline C(92)-H(92B) & 0.9800 \\
\hline C(92)-H(92C) & 0.9800 \\
\hline$C(102)-C(112)$ & $1.517(6)$ \\
\hline$C(102)-C(122)$ & $1.529(6)$ \\
\hline $\mathrm{C}(102)-\mathrm{H}(102)$ & 1.0000 \\
\hline $\mathrm{C}(112)-\mathrm{H}(11 \mathrm{D})$ & 0.9800 \\
\hline $\mathrm{C}(112)-\mathrm{H}(11 \mathrm{E})$ & 0.9800 \\
\hline $\mathrm{C}(112)-\mathrm{H}(11 \mathrm{~F})$ & 0.9800 \\
\hline $\mathrm{C}(122)-\mathrm{H}(12 \mathrm{~A})$ & 0.9800 \\
\hline $\mathrm{C}(122)-\mathrm{H}(12 \mathrm{~B})$ & 0.9800 \\
\hline $\mathrm{C}(122)-\mathrm{H}(12 \mathrm{C})$ & 0.9800 \\
\hline$C(13)-C(23)$ & $1.404(5)$ \\
\hline$C(13)-C(63)$ & $1.411(5)$ \\
\hline$C(23)-C(33)$ & $1.387(5)$ \\
\hline$C(23)-C(73)$ & $1.534(5)$ \\
\hline C(33)-C(43) & $1.385(5)$ \\
\hline $\mathrm{C}(33)-\mathrm{H}(33)$ & 0.9500 \\
\hline$C(43)-C(53)$ & $1.378(5)$ \\
\hline $\mathrm{C}(43)-\mathrm{H}(43)$ & 0.9500 \\
\hline$C(53)-C(63)$ & $1.394(5)$ \\
\hline $\mathrm{C}(53)-\mathrm{H}(53)$ & 0.9500 \\
\hline$C(63)-C(103)$ & $1.525(5)$ \\
\hline$C(73)-C(93)$ & $1.529(6)$ \\
\hline$C(73)-C(83)$ & $1.536(6)$ \\
\hline $\mathrm{C}(73)-\mathrm{H}(73)$ & 1.0000 \\
\hline $\mathrm{C}(83)-\mathrm{H}(83 \mathrm{~A})$ & 0.9800 \\
\hline C(83)-H(83B) & 0.9800 \\
\hline $\mathrm{C}(83)-\mathrm{H}(83 \mathrm{C})$ & 0.9800 \\
\hline C(93)-H(93A) & 0.9800 \\
\hline C(93)-H(93B) & 0.9800 \\
\hline C(93)-H(93C) & 0.9800 \\
\hline C(103)-C(113) & $1.531(5)$ \\
\hline$C(103)-C(123)$ & $1.545(5)$ \\
\hline C(103)-H(103) & 1.0000 \\
\hline $\mathrm{C}(113)-\mathrm{H}(11 \mathrm{G})$ & 0.9800 \\
\hline
\end{tabular}




\begin{tabular}{|c|c|}
\hline $\mathrm{C}(113)-\mathrm{H}(11 \mathrm{H})$ & 0.9800 \\
\hline $\mathrm{C}(113)-\mathrm{H}(11 \mathrm{I})$ & 0.9800 \\
\hline $\mathrm{C}(123)-\mathrm{H}(12 \mathrm{D})$ & 0.9800 \\
\hline $\mathrm{C}(123)-\mathrm{H}(12 \mathrm{E})$ & 0.9800 \\
\hline $\mathrm{C}(123)-\mathrm{H}(12 \mathrm{~F})$ & 0.9800 \\
\hline $\mathrm{O}(14)-\mathrm{C}(34)$ & $1.423(4)$ \\
\hline$C(14)-C(24)$ & $1.341(5)$ \\
\hline$C(14)-C(44)$ & $1.479(5)$ \\
\hline$C(24)-C(34)$ & $1.538(4)$ \\
\hline $\mathrm{C}(24)-\mathrm{H}(24)$ & 0.9500 \\
\hline$C(34)-C(164)$ & $1.536(5)$ \\
\hline$C(34)-C(104)$ & $1.542(4)$ \\
\hline $\mathrm{C}(44)-\mathrm{C}(94)$ & $1.397(5)$ \\
\hline $\mathrm{C}(44)-\mathrm{C}(54)$ & $1.405(5)$ \\
\hline$C(54)-C(64)$ & $1.383(5)$ \\
\hline $\mathrm{C}(54)-\mathrm{H}(54)$ & 0.9500 \\
\hline$C(64)-C(74)$ & $1.387(5)$ \\
\hline $\mathrm{C}(64)-\mathrm{H}(64)$ & 0.9500 \\
\hline$C(74)-C(84)$ & $1.384(5)$ \\
\hline $\mathrm{C}(74)-\mathrm{H}(74)$ & 0.9500 \\
\hline $\mathrm{C}(84)-\mathrm{C}(94)$ & $1.382(5)$ \\
\hline $\mathrm{C}(84)-\mathrm{H}(84)$ & 0.9500 \\
\hline $\mathrm{C}(94)-\mathrm{H}(94)$ & 0.9500 \\
\hline$C(104)-C(154)$ & $1.389(5)$ \\
\hline$C(104)-C(114)$ & $1.395(5)$ \\
\hline$C(114)-C(124)$ & $1.388(5)$ \\
\hline $\mathrm{C}(114)-\mathrm{H}(114)$ & 0.9500 \\
\hline$C(124)-C(134)$ & $1.383(5)$ \\
\hline $\mathrm{C}(124)-\mathrm{H}(124)$ & 0.9500 \\
\hline$C(134)-C(144)$ & $1.380(5)$ \\
\hline $\mathrm{C}(134)-\mathrm{H}(134)$ & 0.9500 \\
\hline$C(144)-C(154)$ & $1.385(5)$ \\
\hline $\mathrm{C}(144)-\mathrm{H}(144)$ & 0.9500 \\
\hline $\mathrm{C}(154)-\mathrm{H}(154)$ & 0.9500 \\
\hline$C(164)-C(174)$ & $1.383(5)$ \\
\hline$C(164)-C(214)$ & $1.384(5)$ \\
\hline$C(174)-C(184)$ & $1.391(5)$ \\
\hline
\end{tabular}




\begin{tabular}{|c|c|}
\hline $\mathrm{C}(174)-\mathrm{H}(174)$ & 0.9500 \\
\hline$C(184)-C(194)$ & $1.385(5)$ \\
\hline $\mathrm{C}(184)-\mathrm{H}(184)$ & 0.9500 \\
\hline$C(194)-C(204)$ & $1.379(6)$ \\
\hline C(194)-H(194) & 0.9500 \\
\hline$C(204)-C(214)$ & $1.388(5)$ \\
\hline $\mathrm{C}(204)-\mathrm{H}(204)$ & 0.9500 \\
\hline $\mathrm{C}(214)-\mathrm{H}(214)$ & 0.9500 \\
\hline $\mathrm{O}(14)-\mathrm{Fe}(01)-\mathrm{N}(21)$ & $115.80(11)$ \\
\hline $\mathrm{O}(14)-\mathrm{Fe}(01)-\mathrm{N}(11)$ & $117.21(10)$ \\
\hline $\mathrm{N}(21)-\mathrm{Fe}(01)-\mathrm{N}(11)$ & $95.94(11)$ \\
\hline $\mathrm{O}(14)-\mathrm{Fe}(01)-\mathrm{C}(14)$ & $89.50(12)$ \\
\hline $\mathrm{N}(21)-\mathrm{Fe}(01)-\mathrm{C}(14)$ & $119.27(12)$ \\
\hline N(11)-Fe(01)-C(14) & $121.11(12)$ \\
\hline $\mathrm{C}(21)-\mathrm{N}(11)-\mathrm{C}(12)$ & $120.3(3)$ \\
\hline $\mathrm{C}(21)-\mathrm{N}(11)-\mathrm{Fe}(01)$ & $121.1(2)$ \\
\hline $\mathrm{C}(12)-\mathrm{N}(11)-\mathrm{Fe}(01)$ & $118.5(2)$ \\
\hline $\mathrm{C}(41)-\mathrm{N}(21)-\mathrm{C}(13)$ & $120.3(3)$ \\
\hline $\mathrm{C}(41)-\mathrm{N}(21)-\mathrm{Fe}(01)$ & $120.8(2)$ \\
\hline $\mathrm{C}(13)-\mathrm{N}(21)-\mathrm{Fe}(01)$ & $118.8(2)$ \\
\hline $\mathrm{C}(21)-\mathrm{C}(11)-\mathrm{H}(11 \mathrm{~A})$ & 109.5 \\
\hline $\mathrm{C}(21)-\mathrm{C}(11)-\mathrm{H}(11 \mathrm{~B})$ & 109.5 \\
\hline $\mathrm{H}(11 \mathrm{~A})-\mathrm{C}(11)-\mathrm{H}(11 \mathrm{~B})$ & 109.5 \\
\hline $\mathrm{C}(21)-\mathrm{C}(11)-\mathrm{H}(11 \mathrm{C})$ & 109.5 \\
\hline $\mathrm{H}(11 \mathrm{~A})-\mathrm{C}(11)-\mathrm{H}(11 \mathrm{C})$ & 109.5 \\
\hline $\mathrm{H}(11 \mathrm{~B})-\mathrm{C}(11)-\mathrm{H}(11 \mathrm{C})$ & 109.5 \\
\hline $\mathrm{N}(11)-\mathrm{C}(21)-\mathrm{C}(31)$ & $123.1(3)$ \\
\hline $\mathrm{N}(11)-\mathrm{C}(21)-\mathrm{C}(11)$ & $120.8(3)$ \\
\hline $\mathrm{C}(31)-\mathrm{C}(21)-\mathrm{C}(11)$ & $116.0(3)$ \\
\hline$C(41)-C(31)-C(21)$ & $130.2(3)$ \\
\hline $\mathrm{C}(41)-\mathrm{C}(31)-\mathrm{H}(31)$ & 114.9 \\
\hline $\mathrm{C}(21)-\mathrm{C}(31)-\mathrm{H}(31)$ & 114.9 \\
\hline $\mathrm{N}(21)-\mathrm{C}(41)-\mathrm{C}(31)$ & $123.6(3)$ \\
\hline $\mathrm{N}(21)-\mathrm{C}(41)-\mathrm{C}(51)$ & $119.9(3)$ \\
\hline $\mathrm{C}(31)-\mathrm{C}(41)-\mathrm{C}(51)$ & $116.5(3)$ \\
\hline $\mathrm{C}(41)-\mathrm{C}(51)-\mathrm{H}(51 \mathrm{~A})$ & 109.5 \\
\hline
\end{tabular}




$\begin{array}{ll}\mathrm{C}(41)-\mathrm{C}(51)-\mathrm{H}(51 \mathrm{~B}) & 109.5 \\ \mathrm{H}(51 \mathrm{~A})-\mathrm{C}(51)-\mathrm{H}(51 \mathrm{~B}) & 109.5 \\ \mathrm{C}(41)-\mathrm{C}(51)-\mathrm{H}(51 \mathrm{C}) & 109.5 \\ \mathrm{H}(51 \mathrm{~A})-\mathrm{C}(51)-\mathrm{H}(51 \mathrm{C}) & 109.5 \\ \mathrm{H}(51 \mathrm{~B})-\mathrm{C}(51)-\mathrm{H}(51 \mathrm{C}) & 109.5 \\ \mathrm{C}(62)-\mathrm{C}(12)-\mathrm{C}(22) & 121.6(3) \\ \mathrm{C}(62)-\mathrm{C}(12)-\mathrm{N}(11) & 119.9(3) \\ \mathrm{C}(22)-\mathrm{C}(12)-\mathrm{N}(11) & 118.4(3) \\ \mathrm{C}(32)-\mathrm{C}(22)-\mathrm{C}(12) & 117.5(3) \\ \mathrm{C}(32)-\mathrm{C}(22)-\mathrm{C}(72) & 119.7(3) \\ \mathrm{C}(12)-\mathrm{C}(22)-\mathrm{C}(72) & 122.7(3) \\ \mathrm{C}(42)-\mathrm{C}(32)-\mathrm{C}(22) & 121.8(3) \\ \mathrm{C}(42)-\mathrm{C}(32)-\mathrm{H}(32) & 119.1 \\ \mathrm{C}(22)-\mathrm{C}(32)-\mathrm{H}(32) & 119.1 \\ \mathrm{C}(32)-\mathrm{C}(42)-\mathrm{C}(52) & 119.7(3) \\ \mathrm{C}(32)-\mathrm{C}(42)-\mathrm{H}(42) & 120.2 \\ \mathrm{C}(52)-\mathrm{C}(42)-\mathrm{H}(42) & 120.2 \\ \mathrm{C}(42)-\mathrm{C}(52)-\mathrm{C}(62) & 121.3(3) \\ \mathrm{C}(42)-\mathrm{C}(52)-\mathrm{H}(52) & 119.3 \\ \mathrm{C}(62)-\mathrm{C}(52)-\mathrm{H}(52) & 119.3 \\ \mathrm{C}(52)-\mathrm{C}(62)-\mathrm{C}(12) & 118.2(3) \\ \mathrm{C}(52)-\mathrm{C}(62)-\mathrm{C}(102) & 120.4(3) \\ \mathrm{C}(12)-\mathrm{C}(62)-\mathrm{C}(102) & 121.4(3) \\ \mathrm{C}(22)-\mathrm{C}(72)-\mathrm{C}(82) & 109.8(3) \\ \mathrm{C}(22)-\mathrm{C}(72)-\mathrm{C}(92) & 111.9(3) \\ \mathrm{C}(82)-\mathrm{C}(72)-\mathrm{C}(92) & 111.4(3) \\ \mathrm{C}(22)-\mathrm{C}(72)-\mathrm{H}(72) & 107.9 \\ \mathrm{C}(82)-\mathrm{C}(72)-\mathrm{H}(72) & 107.9 \\ \mathrm{C}(92)-\mathrm{C}(72)-\mathrm{H}(72) & 107.9 \\ \mathrm{C}(72)-\mathrm{C}(82)-\mathrm{H}(82 \mathrm{~A}) & 109.5 \\ \mathrm{C}(72)-\mathrm{C}(82)-\mathrm{H}(82 \mathrm{~B}) & 109.5 \\ \mathrm{H}(82 \mathrm{~A})-\mathrm{C}(82)-\mathrm{H}(82 \mathrm{~B}) & 109.5 \\ \mathrm{C}(72)-\mathrm{C}(82)-\mathrm{H}(82 \mathrm{C}) & 109.5 \\ \mathrm{H}(82 \mathrm{~A})-\mathrm{C}(82)-\mathrm{H}(82 \mathrm{C}) & 109.5 \\ \mathrm{H}(92 \mathrm{~A})-\mathrm{H}(92 \mathrm{~B}) & 109.5 \\ & 109.5 \\ & \end{array}$




\begin{tabular}{|c|c|}
\hline $\mathrm{H}(92 \mathrm{~A})-\mathrm{C}(92)-\mathrm{H}(92 \mathrm{~B})$ & 109.5 \\
\hline $\mathrm{C}(72)-\mathrm{C}(92)-\mathrm{H}(92 \mathrm{C})$ & 109.5 \\
\hline $\mathrm{H}(92 \mathrm{~A})-\mathrm{C}(92)-\mathrm{H}(92 \mathrm{C})$ & 109.5 \\
\hline $\mathrm{H}(92 \mathrm{~B})-\mathrm{C}(92)-\mathrm{H}(92 \mathrm{C})$ & 109.5 \\
\hline$C(112)-C(102)-C(62)$ & $113.3(3)$ \\
\hline$C(112)-C(102)-C(122)$ & $110.6(3)$ \\
\hline$C(62)-C(102)-C(122)$ & $111.2(3)$ \\
\hline $\mathrm{C}(112)-\mathrm{C}(102)-\mathrm{H}(102)$ & 107.1 \\
\hline $\mathrm{C}(62)-\mathrm{C}(102)-\mathrm{H}(102)$ & 107.1 \\
\hline $\mathrm{C}(122)-\mathrm{C}(102)-\mathrm{H}(102)$ & 107.1 \\
\hline $\mathrm{C}(102)-\mathrm{C}(112)-\mathrm{H}(11 \mathrm{D})$ & 109.5 \\
\hline $\mathrm{C}(102)-\mathrm{C}(112)-\mathrm{H}(11 \mathrm{E})$ & 109.5 \\
\hline $\mathrm{H}(11 \mathrm{D})-\mathrm{C}(112)-\mathrm{H}(11 \mathrm{E})$ & 109.5 \\
\hline $\mathrm{C}(102)-\mathrm{C}(112)-\mathrm{H}(11 \mathrm{~F})$ & 109.5 \\
\hline $\mathrm{H}(11 \mathrm{D})-\mathrm{C}(112)-\mathrm{H}(11 \mathrm{~F})$ & 109.5 \\
\hline $\mathrm{H}(11 \mathrm{E})-\mathrm{C}(112)-\mathrm{H}(11 \mathrm{~F})$ & 109.5 \\
\hline $\mathrm{C}(102)-\mathrm{C}(122)-\mathrm{H}(12 \mathrm{~A})$ & 109.5 \\
\hline $\mathrm{C}(102)-\mathrm{C}(122)-\mathrm{H}(12 \mathrm{~B})$ & 109.5 \\
\hline $\mathrm{H}(12 \mathrm{~A})-\mathrm{C}(122)-\mathrm{H}(12 \mathrm{~B})$ & 109.5 \\
\hline $\mathrm{C}(102)-\mathrm{C}(122)-\mathrm{H}(12 \mathrm{C})$ & 109.5 \\
\hline $\mathrm{H}(12 \mathrm{~A})-\mathrm{C}(122)-\mathrm{H}(12 \mathrm{C})$ & 109.5 \\
\hline $\mathrm{H}(12 \mathrm{~B})-\mathrm{C}(122)-\mathrm{H}(12 \mathrm{C})$ & 109.5 \\
\hline $\mathrm{C}(23)-\mathrm{C}(13)-\mathrm{C}(63)$ & $120.9(3)$ \\
\hline $\mathrm{C}(23)-\mathrm{C}(13)-\mathrm{N}(21)$ & $118.5(3)$ \\
\hline $\mathrm{C}(63)-\mathrm{C}(13)-\mathrm{N}(21)$ & $120.6(3)$ \\
\hline $\mathrm{C}(33)-\mathrm{C}(23)-\mathrm{C}(13)$ & 118.7(3) \\
\hline$C(33)-C(23)-C(73)$ & $120.1(3)$ \\
\hline$C(13)-C(23)-C(73)$ & 121.1(3) \\
\hline$C(43)-C(33)-C(23)$ & 121.1(3) \\
\hline $\mathrm{C}(43)-\mathrm{C}(33)-\mathrm{H}(33)$ & 119.5 \\
\hline $\mathrm{C}(23)-\mathrm{C}(33)-\mathrm{H}(33)$ & 119.5 \\
\hline$C(53)-C(43)-C(33)$ & $119.8(3)$ \\
\hline $\mathrm{C}(53)-\mathrm{C}(43)-\mathrm{H}(43)$ & 120.1 \\
\hline $\mathrm{C}(33)-\mathrm{C}(43)-\mathrm{H}(43)$ & 120.1 \\
\hline$C(43)-C(53)-C(63)$ & $121.6(3)$ \\
\hline $\mathrm{C}(43)-\mathrm{C}(53)-\mathrm{H}(53)$ & 119.2 \\
\hline $\mathrm{C}(63)-\mathrm{C}(53)-\mathrm{H}(53)$ & 119.2 \\
\hline
\end{tabular}




\begin{tabular}{|c|c|}
\hline$C(53)-C(63)-C(13)$ & $117.9(3)$ \\
\hline$C(53)-C(63)-C(103)$ & $119.2(3)$ \\
\hline$C(13)-C(63)-C(103)$ & $122.9(3)$ \\
\hline $\mathrm{C}(93)-\mathrm{C}(73)-\mathrm{C}(23)$ & 111.2(3) \\
\hline$C(93)-C(73)-C(83)$ & $111.0(3)$ \\
\hline$C(23)-C(73)-C(83)$ & $113.1(3)$ \\
\hline $\mathrm{C}(93)-\mathrm{C}(73)-\mathrm{H}(73)$ & 107.1 \\
\hline $\mathrm{C}(23)-\mathrm{C}(73)-\mathrm{H}(73)$ & 107.1 \\
\hline $\mathrm{C}(83)-\mathrm{C}(73)-\mathrm{H}(73)$ & 107.1 \\
\hline $\mathrm{C}(73)-\mathrm{C}(83)-\mathrm{H}(83 \mathrm{~A})$ & 109.5 \\
\hline $\mathrm{C}(73)-\mathrm{C}(83)-\mathrm{H}(83 \mathrm{~B})$ & 109.5 \\
\hline $\mathrm{H}(83 \mathrm{~A})-\mathrm{C}(83)-\mathrm{H}(83 \mathrm{~B})$ & 109.5 \\
\hline $\mathrm{C}(73)-\mathrm{C}(83)-\mathrm{H}(83 \mathrm{C})$ & 109.5 \\
\hline $\mathrm{H}(83 \mathrm{~A})-\mathrm{C}(83)-\mathrm{H}(83 \mathrm{C})$ & 109.5 \\
\hline $\mathrm{H}(83 \mathrm{~B})-\mathrm{C}(83)-\mathrm{H}(83 \mathrm{C})$ & 109.5 \\
\hline $\mathrm{C}(73)-\mathrm{C}(93)-\mathrm{H}(93 \mathrm{~A})$ & 109.5 \\
\hline $\mathrm{C}(73)-\mathrm{C}(93)-\mathrm{H}(93 \mathrm{~B})$ & 109.5 \\
\hline $\mathrm{H}(93 \mathrm{~A})-\mathrm{C}(93)-\mathrm{H}(93 \mathrm{~B})$ & 109.5 \\
\hline C(73)-C(93)-H(93C) & 109.5 \\
\hline $\mathrm{H}(93 \mathrm{~A})-\mathrm{C}(93)-\mathrm{H}(93 \mathrm{C})$ & 109.5 \\
\hline $\mathrm{H}(93 \mathrm{~B})-\mathrm{C}(93)-\mathrm{H}(93 \mathrm{C})$ & 109.5 \\
\hline$C(63)-C(103)-C(113)$ & $110.4(3)$ \\
\hline$C(63)-C(103)-C(123)$ & $112.6(3)$ \\
\hline$C(113)-C(103)-C(123)$ & $109.8(3)$ \\
\hline $\mathrm{C}(63)-\mathrm{C}(103)-\mathrm{H}(103)$ & 108.0 \\
\hline $\mathrm{C}(113)-\mathrm{C}(103)-\mathrm{H}(103)$ & 108.0 \\
\hline $\mathrm{C}(123)-\mathrm{C}(103)-\mathrm{H}(103)$ & 108.0 \\
\hline $\mathrm{C}(103)-\mathrm{C}(113)-\mathrm{H}(11 \mathrm{G})$ & 109.5 \\
\hline $\mathrm{C}(103)-\mathrm{C}(113)-\mathrm{H}(11 \mathrm{H})$ & 109.5 \\
\hline $\mathrm{H}(11 \mathrm{G})-\mathrm{C}(113)-\mathrm{H}(11 \mathrm{H})$ & 109.5 \\
\hline $\mathrm{C}(103)-\mathrm{C}(113)-\mathrm{H}(11 \mathrm{I})$ & 109.5 \\
\hline $\mathrm{H}(11 \mathrm{G})-\mathrm{C}(113)-\mathrm{H}(11 \mathrm{I})$ & 109.5 \\
\hline $\mathrm{H}(11 \mathrm{H})-\mathrm{C}(113)-\mathrm{H}(11 \mathrm{I})$ & 109.5 \\
\hline $\mathrm{C}(103)-\mathrm{C}(123)-\mathrm{H}(12 \mathrm{D})$ & 109.5 \\
\hline $\mathrm{C}(103)-\mathrm{C}(123)-\mathrm{H}(12 \mathrm{E})$ & 109.5 \\
\hline $\mathrm{H}(12 \mathrm{D})-\mathrm{C}(123)-\mathrm{H}(12 \mathrm{E})$ & 109.5 \\
\hline $\mathrm{C}(103)-\mathrm{C}(123)-\mathrm{H}(12 \mathrm{~F})$ & 109.5 \\
\hline
\end{tabular}




$\begin{array}{ll}\mathrm{H}(12 \mathrm{D})-\mathrm{C}(123)-\mathrm{H}(12 \mathrm{~F}) & 109.5 \\ \mathrm{H}(12 \mathrm{E})-\mathrm{C}(123)-\mathrm{H}(12 \mathrm{~F}) & 109.5 \\ \mathrm{C}(34)-\mathrm{O}(14)-\mathrm{Fe}(01) & 114.01(19) \\ \mathrm{C}(24)-\mathrm{C}(14)-\mathrm{C}(44) & 125.4(3) \\ \mathrm{C}(24)-\mathrm{C}(14)-\mathrm{Fe}(01) & 104.9(2) \\ \mathrm{C}(44)-\mathrm{C}(14)-\mathrm{Fe}(01) & 129.7(2) \\ \mathrm{C}(14)-\mathrm{C}(24)-\mathrm{C}(34) & 121.5(3) \\ \mathrm{C}(14)-\mathrm{C}(24)-\mathrm{H}(24) & 119.2 \\ \mathrm{C}(34)-\mathrm{C}(24)-\mathrm{H}(24) & 119.2 \\ \mathrm{O}(14)-\mathrm{C}(34)-\mathrm{C}(164) & 106.7(2) \\ \mathrm{O}(14)-\mathrm{C}(34)-\mathrm{C}(24) & 109.9(3) \\ \mathrm{C}(164)-\mathrm{C}(34)-\mathrm{C}(24) & 112.9(3) \\ \mathrm{O}(14)-\mathrm{C}(34)-\mathrm{C}(104) & 109.8(3) \\ \mathrm{C}(164)-\mathrm{C}(34)-\mathrm{C}(104) & 111.8(3) \\ \mathrm{C}(24)-\mathrm{C}(34)-\mathrm{C}(104) & 105.9(2) \\ \mathrm{C}(94)-\mathrm{C}(44)-\mathrm{C}(54) & 116.8(3) \\ \mathrm{C}(94)-\mathrm{C}(44)-\mathrm{C}(14) & 119.7(3) \\ \mathrm{C}(54)-\mathrm{C}(44)-\mathrm{C}(14) & 123.4(3) \\ \mathrm{C}(64)-\mathrm{C}(54)-\mathrm{C}(44) & 121.5(3) \\ \mathrm{C}(64)-\mathrm{C}(54)-\mathrm{H}(54) & 119.3 \\ \mathrm{C}(44)-\mathrm{C}(54)-\mathrm{H}(54) & 119.3 \\ \mathrm{C}(54)-\mathrm{C}(64)-\mathrm{C}(74) & 120.2(3) \\ \mathrm{C}(54)-\mathrm{C}(64)-\mathrm{H}(64) & 119.9 \\ \mathrm{C}(74)-\mathrm{C}(64)-\mathrm{H}(64) & 119.9 \\ \mathrm{C}(84)-\mathrm{C}(74)-\mathrm{C}(64) & 119.5(3) \\ \mathrm{C}(84)-\mathrm{C}(74)-\mathrm{H}(74) & 120.2 \\ \mathrm{C}(64)-\mathrm{C}(74)-\mathrm{H}(74) & 120.2 \\ \mathrm{C}(94)-\mathrm{C}(84)-\mathrm{C}(74) & 119.9(3) \\ \mathrm{C}(94)-\mathrm{C}(84)-\mathrm{H}(84) & 120.0 \\ \mathrm{C}(74)-\mathrm{C}(84)-\mathrm{H}(84) & 120.0 \\ \mathrm{C}(84)-\mathrm{C}(94)-\mathrm{C}(44) & 122.0(3) \\ \mathrm{C}(84)-\mathrm{C}(94)-\mathrm{H}(94) & 119.0 \\ \mathrm{C}(44)-\mathrm{C}(94)-\mathrm{H}(94) & 119.0 \\ \mathrm{C}(154)-\mathrm{C}(104)-\mathrm{C}(114) & 118.6(3) \\ \mathrm{C}(154)-\mathrm{C}(104)-\mathrm{C}(34) & 121.0(3) \\ & 120.4(3) \\ \mathrm{C}(104)-\mathrm{C}(34) & \\ & \\ & \\ \mathrm{C}(114)-\mathrm{C}(104) & 20.2(3) \\ & \end{array}$




$\begin{array}{ll}\mathrm{C}(124)-\mathrm{C}(114)-\mathrm{H}(114) & 119.9 \\ \mathrm{C}(104)-\mathrm{C}(114)-\mathrm{H}(114) & 119.9 \\ \mathrm{C}(134)-\mathrm{C}(124)-\mathrm{C}(114) & 120.5(3) \\ \mathrm{C}(134)-\mathrm{C}(124)-\mathrm{H}(124) & 119.8 \\ \mathrm{C}(114)-\mathrm{C}(124)-\mathrm{H}(124) & 119.8 \\ \mathrm{C}(144)-\mathrm{C}(134)-\mathrm{C}(124) & 119.7(3) \\ \mathrm{C}(144)-\mathrm{C}(134)-\mathrm{H}(134) & 120.2 \\ \mathrm{C}(124)-\mathrm{C}(134)-\mathrm{H}(134) & 120.2 \\ \mathrm{C}(134)-\mathrm{C}(144)-\mathrm{C}(154) & 120.0(3) \\ \mathrm{C}(134)-\mathrm{C}(144)-\mathrm{H}(144) & 120.0 \\ \mathrm{C}(154)-\mathrm{C}(144)-\mathrm{H}(144) & 120.0 \\ \mathrm{C}(144)-\mathrm{C}(154)-\mathrm{C}(104) & 121.0(3) \\ \mathrm{C}(144)-\mathrm{C}(154)-\mathrm{H}(154) & 119.5 \\ \mathrm{C}(104)-\mathrm{C}(154)-\mathrm{H}(154) & 119.5 \\ \mathrm{C}(174)-\mathrm{C}(164)-\mathrm{C}(214) & 118.3(3) \\ \mathrm{C}(174)-\mathrm{C}(164)-\mathrm{C}(34) & 119.4(3) \\ \mathrm{C}(214)-\mathrm{C}(164)-\mathrm{C}(34) & 122.2(3) \\ \mathrm{C}(164)-\mathrm{C}(174)-\mathrm{C}(184) & 121.4(3) \\ \mathrm{C}(164)-\mathrm{C}(174)-\mathrm{H}(174) & 119.3 \\ \mathrm{C}(184)-\mathrm{C}(174)-\mathrm{H}(174) & 119.3 \\ \mathrm{C}(194)-\mathrm{C}(184)-\mathrm{C}(174) & 119.8(3) \\ \mathrm{C}(194)-\mathrm{C}(184)-\mathrm{H}(184) & 120.1 \\ \mathrm{C}(174)-\mathrm{C}(184)-\mathrm{H}(184) & 120.1 \\ \mathrm{C}(204)-\mathrm{C}(194)-\mathrm{C}(184) & 118.9(3) \\ \mathrm{C}(204)-\mathrm{C}(194)-\mathrm{H}(194) & 120.5 \\ \mathrm{C}(184)-\mathrm{C}(194)-\mathrm{H}(194) & 120.5 \\ \mathrm{C}(194)-\mathrm{C}(204)-\mathrm{C}(214) & 121.1(3) \\ \mathrm{C}(194)-\mathrm{C}(204)-\mathrm{H}(204) & 119.5 \\ \mathrm{C}(214)-\mathrm{C}(204)-\mathrm{H}(204) & 119.5 \\ \mathrm{C}(164)-\mathrm{C}(214)-\mathrm{C}(204) & 120.5(3) \\ \mathrm{C}(164)-\mathrm{C}(214)-\mathrm{H}(214) & 119.8 \\ & 119.8 \\ & \end{array}$


Table S-27. Anisotropic displacement parameters $\left(\AA^{2} \times 10^{3}\right)$ for 6 . The anisotropic displacement factor exponent takes the form: $-2 \pi^{2}\left[h^{2} a^{* 2} U^{11}+\ldots+2 h k a^{*} b^{*} U^{12}\right]$

\begin{tabular}{|c|c|c|c|c|c|c|}
\hline & $\mathrm{U}^{11}$ & $\mathrm{U}^{22}$ & $\mathrm{U}^{33}$ & $\mathrm{U}^{23}$ & $\mathrm{U}^{13}$ & $\mathrm{U}^{12}$ \\
\hline $\mathrm{Fe}(01)$ & $24(1)$ & $21(1)$ & $26(1)$ & $-1(1)$ & $0(1)$ & $-1(1)$ \\
\hline $\mathrm{N}(11)$ & $28(1)$ & $24(2)$ & $23(1)$ & $1(1)$ & $-2(1)$ & $-2(1)$ \\
\hline $\mathrm{N}(21)$ & $26(1)$ & $25(2)$ & $31(2)$ & $0(1)$ & $0(1)$ & $1(1)$ \\
\hline$C(11)$ & $39(2)$ & $25(2)$ & $36(2)$ & $3(2)$ & $-2(2)$ & $-1(2)$ \\
\hline $\mathrm{C}(21)$ & $36(2)$ & $22(2)$ & $23(2)$ & $0(1)$ & $-4(1)$ & $0(1)$ \\
\hline $\mathrm{C}(31)$ & $36(2)$ & $22(2)$ & $38(2)$ & $4(1)$ & $-5(2)$ & $5(1)$ \\
\hline $\mathrm{C}(41)$ & $29(2)$ & $28(2)$ & $36(2)$ & $-1(1)$ & $-3(1)$ & $3(1)$ \\
\hline $\mathrm{C}(51)$ & $33(2)$ & $33(2)$ & $69(3)$ & $-3(2)$ & $4(2)$ & $8(2)$ \\
\hline$C(12)$ & $29(2)$ & $20(2)$ & $31(2)$ & $3(1)$ & $-3(1)$ & $-4(1)$ \\
\hline $\mathrm{C}(22)$ & $34(2)$ & $27(2)$ & $34(2)$ & $4(1)$ & $-5(2)$ & $-2(1)$ \\
\hline$C(32)$ & $34(2)$ & $39(2)$ & $36(2)$ & $7(2)$ & $-10(2)$ & $-7(2)$ \\
\hline $\mathrm{C}(42)$ & $28(2)$ & $44(2)$ & $50(2)$ & $9(2)$ & $-2(2)$ & $-6(2)$ \\
\hline$C(52)$ & $36(2)$ & $37(2)$ & $44(2)$ & $5(2)$ & $9(2)$ & $-5(2)$ \\
\hline$C(62)$ & $34(2)$ & $26(2)$ & $32(2)$ & $1(2)$ & $3(1)$ & $-7(2)$ \\
\hline$C(72)$ & $40(2)$ & $40(2)$ & $28(2)$ & $-2(2)$ & $-5(2)$ & $2(2)$ \\
\hline $\mathrm{C}(82)$ & $41(2)$ & $52(3)$ & $33(2)$ & $1(2)$ & $-2(2)$ & $-5(2)$ \\
\hline $\mathrm{C}(92)$ & $69(3)$ & $43(2)$ & $38(2)$ & $-7(2)$ & $-11(2)$ & $5(2)$ \\
\hline$C(102)$ & $37(2)$ & $38(2)$ & $34(2)$ & $-4(2)$ & $5(2)$ & $-10(2)$ \\
\hline$C(112)$ & $77(3)$ & $40(2)$ & $39(2)$ & $-1(2)$ & $10(2)$ & $0(2)$ \\
\hline$C(122)$ & $53(2)$ & $50(2)$ & $33(2)$ & $-4(2)$ & $1(2)$ & $10(2)$ \\
\hline$C(13)$ & $24(2)$ & $24(2)$ & $34(2)$ & $-2(1)$ & $3(1)$ & $1(1)$ \\
\hline $\mathrm{C}(23)$ & $29(2)$ & $34(2)$ & $31(2)$ & $-6(2)$ & $4(1)$ & $2(1)$ \\
\hline $\mathrm{C}(33)$ & $40(2)$ & $43(2)$ & $32(2)$ & $2(2)$ & $8(2)$ & $-3(2)$ \\
\hline C(43) & $35(2)$ & $44(2)$ & $44(2)$ & $3(2)$ & $9(2)$ & $-11(2)$ \\
\hline $\mathrm{C}(53)$ & $28(2)$ & $39(2)$ & $41(2)$ & $1(2)$ & $-1(2)$ & $-6(2)$ \\
\hline$C(63)$ & $24(2)$ & $28(2)$ & $37(2)$ & $-1(1)$ & $0(1)$ & $1(1)$ \\
\hline$C(73)$ & $33(2)$ & $42(2)$ & $30(2)$ & $-9(2)$ & $4(1)$ & $-6(2)$ \\
\hline$C(83)$ & $49(2)$ & $62(3)$ & $33(2)$ & $-2(2)$ & $-7(2)$ & $-3(2)$ \\
\hline $\mathrm{C}(93)$ & $50(2)$ & $53(3)$ & $50(2)$ & $-20(2)$ & $9(2)$ & $-6(2)$ \\
\hline$C(103)$ & $28(2)$ & $37(2)$ & $37(2)$ & $5(2)$ & $-4(1)$ & $-4(1)$ \\
\hline$C(113)$ & $39(2)$ & $46(2)$ & $32(2)$ & $0(2)$ & $-3(2)$ & $-5(2)$ \\
\hline$C(123)$ & $37(2)$ & $50(3)$ & $50(2)$ & $10(2)$ & $-7(2)$ & $2(2)$ \\
\hline
\end{tabular}




\begin{tabular}{lllllll}
$\mathrm{O}(14)$ & $27(1)$ & $23(1)$ & $27(1)$ & $-4(1)$ & $0(1)$ & $2(1)$ \\
$\mathrm{C}(14)$ & $22(2)$ & $28(2)$ & $28(2)$ & $-2(1)$ & $1(1)$ & $-5(1)$ \\
$\mathrm{C}(24)$ & $24(2)$ & $25(2)$ & $28(2)$ & $-2(1)$ & $0(1)$ & $-1(1)$ \\
$\mathrm{C}(34)$ & $26(2)$ & $24(2)$ & $26(2)$ & $-2(1)$ & $-1(1)$ & $-2(1)$ \\
$\mathrm{C}(44)$ & $25(2)$ & $26(2)$ & $28(2)$ & $-2(1)$ & $1(1)$ & $-5(1)$ \\
$\mathrm{C}(54)$ & $29(2)$ & $30(2)$ & $32(2)$ & $-4(1)$ & $1(1)$ & $-1(1)$ \\
$\mathrm{C}(64)$ & $32(2)$ & $38(2)$ & $28(2)$ & $-7(2)$ & $3(1)$ & $-5(2)$ \\
$\mathrm{C}(74)$ & $33(2)$ & $36(2)$ & $25(2)$ & $0(1)$ & $-1(1)$ & $-8(2)$ \\
$\mathrm{C}(84)$ & $32(2)$ & $32(2)$ & $35(2)$ & $5(2)$ & $0(1)$ & $-2(2)$ \\
$\mathrm{C}(94)$ & $32(2)$ & $28(2)$ & $29(2)$ & $-2(1)$ & $3(1)$ & $-3(1)$ \\
$\mathrm{C}(104)$ & $27(2)$ & $28(2)$ & $22(2)$ & $3(1)$ & $1(1)$ & $0(1)$ \\
$\mathrm{C}(114)$ & $28(2)$ & $28(2)$ & $33(2)$ & $-1(1)$ & $1(1)$ & $-1(1)$ \\
$\mathrm{C}(124)$ & $29(2)$ & $35(2)$ & $37(2)$ & $-1(2)$ & $3(2)$ & $-5(2)$ \\
$\mathrm{C}(134)$ & $28(2)$ & $38(2)$ & $31(2)$ & $2(2)$ & $4(1)$ & $4(2)$ \\
$\mathrm{C}(144)$ & $34(2)$ & $29(2)$ & $36(2)$ & $-2(2)$ & $4(2)$ & $4(2)$ \\
$\mathrm{C}(154)$ & $30(2)$ & $27(2)$ & $32(2)$ & $-2(1)$ & $2(1)$ & $-1(1)$ \\
$\mathrm{C}(164)$ & $28(2)$ & $21(2)$ & $32(2)$ & $-2(1)$ & $4(1)$ & $2(1)$ \\
$\mathrm{C}(174)$ & $33(2)$ & $32(2)$ & $29(2)$ & $-2(2)$ & $-1(1)$ & $-5(2)$ \\
$\mathrm{C}(184)$ & $42(2)$ & $37(2)$ & $30(2)$ & $4(2)$ & $4(2)$ & $-2(2)$ \\
$\mathrm{C}(194)$ & $34(2)$ & $38(2)$ & $49(2)$ & $7(2)$ & $10(2)$ & $-5(2)$ \\
$\mathrm{C}(204)$ & $28(2)$ & $44(2)$ & $63(3)$ & $13(2)$ & $-5(2)$ & $-9(2)$ \\
$\mathrm{C}(214)$ & $29(2)$ & $37(2)$ & $48(2)$ & $11(2)$ & $-5(2)$ & $-1(2)$ \\
& & & & & & \\
\hline
\end{tabular}


Table S-28. Hydrogen coordinates ( $\left.\times 10^{4}\right)$ and isotropic displacement parameters $\left(\AA^{2} \times 10^{3}\right)$ for 6 .

\begin{tabular}{|c|c|c|c|c|}
\hline & $\mathrm{x}$ & $\mathrm{y}$ & $\mathrm{z}$ & $\mathrm{U}(\mathrm{eq})$ \\
\hline $\mathrm{H}(11 \mathrm{~A})$ & 8314 & 9566 & 7697 & 51 \\
\hline $\mathrm{H}(11 \mathrm{~B})$ & 7926 & 9687 & 6580 & 51 \\
\hline $\mathrm{H}(11 \mathrm{C})$ & 8987 & 9044 & 6925 & 51 \\
\hline $\mathrm{H}(31)$ & 6364 & 8862 & 6877 & 39 \\
\hline $\mathrm{H}(51 \mathrm{~A})$ & 4422 & 7606 & 6770 & 68 \\
\hline $\mathrm{H}(51 \mathrm{~B})$ & 4897 & 8555 & 7525 & 68 \\
\hline $\mathrm{H}(51 \mathrm{C})$ & 4597 & 7301 & 7886 & 68 \\
\hline $\mathrm{H}(32)$ & 11133 & 7356 & 9231 & 45 \\
\hline $\mathrm{H}(42)$ & 12176 & 6835 & 8090 & 49 \\
\hline $\mathrm{H}(52)$ & 11459 & 6472 & 6524 & 47 \\
\hline $\mathrm{H}(72)$ & 8380 & 7650 & 8854 & 44 \\
\hline $\mathrm{H}(82 \mathrm{~A})$ & 8856 & 5840 & 9520 & 64 \\
\hline $\mathrm{H}(82 \mathrm{~B})$ & 8703 & 6721 & 10367 & 64 \\
\hline $\mathrm{H}(82 \mathrm{C})$ & 9836 & 6394 & 10145 & 64 \\
\hline $\mathrm{H}(92 \mathrm{~A})$ & 10134 & 8596 & 10009 & 77 \\
\hline $\mathrm{H}(92 \mathrm{~B})$ & 8963 & 8866 & 10141 & 77 \\
\hline $\mathrm{H}(92 \mathrm{C})$ & 9427 & 9313 & 9209 & 77 \\
\hline $\mathrm{H}(102)$ & 8808 & 6199 & 5713 & 43 \\
\hline $\mathrm{H}(11 \mathrm{D})$ & 10772 & 5958 & 5076 & 78 \\
\hline $\mathrm{H}(11 \mathrm{E})$ & 9716 & 5410 & 4586 & 78 \\
\hline $\mathrm{H}(11 \mathrm{~F})$ & 10231 & 4941 & 5601 & 78 \\
\hline $\mathrm{H}(12 \mathrm{~A})$ & 8983 & 8192 & 5435 & 68 \\
\hline $\mathrm{H}(12 \mathrm{~B})$ & 9057 & 7478 & 4473 & 68 \\
\hline $\mathrm{H}(12 \mathrm{C})$ & 10078 & 7974 & 5075 & 68 \\
\hline $\mathrm{H}(33)$ & 4877 & 3991 & 9576 & 46 \\
\hline $\mathrm{H}(43)$ & 3480 & 3375 & 8517 & 49 \\
\hline $\mathrm{H}(53)$ & 3343 & 3915 & 6923 & 43 \\
\hline $\mathrm{H}(73)$ & 7008 & 5600 & 8991 & 42 \\
\hline $\mathrm{H}(83 \mathrm{~A})$ & 6251 & 4434 & 10550 & 73 \\
\hline $\mathrm{H}(83 \mathrm{~B})$ & 7416 & 4821 & 10503 & 73 \\
\hline $\mathrm{H}(83 \mathrm{C})$ & 6919 & 3808 & 9823 & 73 \\
\hline
\end{tabular}




\begin{tabular}{|c|c|c|c|c|}
\hline $\mathrm{H}(93 \mathrm{~A})$ & 5985 & 7169 & 9308 & 77 \\
\hline $\mathrm{H}(93 \mathrm{~B})$ & 6726 & 6849 & 10260 & 77 \\
\hline $\mathrm{H}(93 \mathrm{C})$ & 5543 & 6473 & 10151 & 77 \\
\hline $\mathrm{H}(103)$ & 5031 & 5840 & 5859 & 41 \\
\hline $\mathrm{H}(11 \mathrm{G})$ & 5395 & 3846 & 5630 & 60 \\
\hline $\mathrm{H}(11 \mathrm{H})$ & 4730 & 4382 & 4707 & 60 \\
\hline $\mathrm{H}(11 \mathrm{I})$ & 4189 & 3586 & 5428 & 60 \\
\hline $\mathrm{H}(12 \mathrm{D})$ & 2911 & 5142 & 5624 & 70 \\
\hline $\mathrm{H}(12 \mathrm{E})$ & 3431 & 5971 & 4911 & 70 \\
\hline $\mathrm{H}(12 \mathrm{~F})$ & 3290 & 6408 & 5965 & 70 \\
\hline $\mathrm{H}(24)$ & 7899 & 2834 & 5893 & 31 \\
\hline $\mathrm{H}(54)$ & 7973 & 3370 & 4354 & 37 \\
\hline $\mathrm{H}(64)$ & 7567 & 3852 & 2753 & 39 \\
\hline $\mathrm{H}(74)$ & 6626 & 5529 & 2318 & 38 \\
\hline $\mathrm{H}(84)$ & 6143 & 6753 & 3506 & 40 \\
\hline $\mathrm{H}(94)$ & 6579 & 6294 & 5105 & 36 \\
\hline $\mathrm{H}(114)$ & 9880 & 4627 & 7861 & 36 \\
\hline $\mathrm{H}(124)$ & 11623 & 4180 & 7926 & 41 \\
\hline $\mathrm{H}(134)$ & 12143 & 2291 & 7661 & 39 \\
\hline $\mathrm{H}(144)$ & 10915 & 854 & 7272 & 39 \\
\hline $\mathrm{H}(154)$ & 9174 & 1306 & 7144 & 36 \\
\hline $\mathrm{H}(174)$ & 8581 & 2159 & 9017 & 38 \\
\hline $\mathrm{H}(184)$ & 7616 & 831 & 9778 & 44 \\
\hline H(194) & 5998 & 248 & 9042 & 48 \\
\hline $\mathrm{H}(204)$ & 5375 & 1011 & 7554 & 55 \\
\hline $\mathrm{H}(214)$ & 6331 & 2355 & 6806 & 47 \\
\hline
\end{tabular}


Table S-29. Torsion angles [ $\left.{ }^{\circ}\right]$ for $\left[\mathrm{L}^{\mathrm{Me}} \mathrm{Fe}\left(\mathrm{OCPh}_{2} \mathrm{CHCPh}\right)\right](\mathbf{6})$.

\begin{tabular}{|c|c|}
\hline $\mathrm{C}(12)-\mathrm{N}(11)-\mathrm{C}(21)-\mathrm{C}(31)$ & $-166.8(3)$ \\
\hline $\mathrm{Fe}(01)-\mathrm{N}(11)-\mathrm{C}(21)-\mathrm{C}(31)$ & $9.9(4)$ \\
\hline $\mathrm{C}(12)-\mathrm{N}(11)-\mathrm{C}(21)-\mathrm{C}(11)$ & $9.5(4)$ \\
\hline $\mathrm{Fe}(01)-\mathrm{N}(11)-\mathrm{C}(21)-\mathrm{C}(11)$ & $-173.9(2)$ \\
\hline $\mathrm{N}(11)-\mathrm{C}(21)-\mathrm{C}(31)-\mathrm{C}(41)$ & $11.1(6)$ \\
\hline $\mathrm{C}(11)-\mathrm{C}(21)-\mathrm{C}(31)-\mathrm{C}(41)$ & $-165.3(4)$ \\
\hline $\mathrm{C}(13)-\mathrm{N}(21)-\mathrm{C}(41)-\mathrm{C}(31)$ & $173.9(3)$ \\
\hline $\mathrm{Fe}(01)-\mathrm{N}(21)-\mathrm{C}(41)-\mathrm{C}(31)$ & $-8.7(5)$ \\
\hline $\mathrm{C}(13)-\mathrm{N}(21)-\mathrm{C}(41)-\mathrm{C}(51)$ & $-5.0(5)$ \\
\hline $\mathrm{Fe}(01)-\mathrm{N}(21)-\mathrm{C}(41)-\mathrm{C}(51)$ & $172.5(3)$ \\
\hline $\mathrm{C}(21)-\mathrm{C}(31)-\mathrm{C}(41)-\mathrm{N}(21)$ & $-11.8(6)$ \\
\hline $\mathrm{C}(21)-\mathrm{C}(31)-\mathrm{C}(41)-\mathrm{C}(51)$ & $167.1(4)$ \\
\hline $\mathrm{C}(21)-\mathrm{N}(11)-\mathrm{C}(12)-\mathrm{C}(62)$ & $-107.3(4)$ \\
\hline $\mathrm{Fe}(01)-\mathrm{N}(11)-\mathrm{C}(12)-\mathrm{C}(62)$ & $76.0(3)$ \\
\hline $\mathrm{C}(21)-\mathrm{N}(11)-\mathrm{C}(12)-\mathrm{C}(22)$ & $77.2(4)$ \\
\hline $\mathrm{Fe}(01)-\mathrm{N}(11)-\mathrm{C}(12)-\mathrm{C}(22)$ & $-99.5(3)$ \\
\hline $\mathrm{C}(62)-\mathrm{C}(12)-\mathrm{C}(22)-\mathrm{C}(32)$ & $0.8(5)$ \\
\hline $\mathrm{N}(11)-\mathrm{C}(12)-\mathrm{C}(22)-\mathrm{C}(32)$ & $176.2(3)$ \\
\hline$C(62)-C(12)-C(22)-C(72)$ & $-177.3(3)$ \\
\hline $\mathrm{N}(11)-\mathrm{C}(12)-\mathrm{C}(22)-\mathrm{C}(72)$ & $-1.9(5)$ \\
\hline $\mathrm{C}(12)-\mathrm{C}(22)-\mathrm{C}(32)-\mathrm{C}(42)$ & $-1.2(5)$ \\
\hline $\mathrm{C}(72)-\mathrm{C}(22)-\mathrm{C}(32)-\mathrm{C}(42)$ & $177.0(4)$ \\
\hline $\mathrm{C}(22)-\mathrm{C}(32)-\mathrm{C}(42)-\mathrm{C}(52)$ & $1.0(6)$ \\
\hline $\mathrm{C}(32)-\mathrm{C}(42)-\mathrm{C}(52)-\mathrm{C}(62)$ & $-0.5(6)$ \\
\hline $\mathrm{C}(42)-\mathrm{C}(52)-\mathrm{C}(62)-\mathrm{C}(12)$ & $0.2(5)$ \\
\hline $\mathrm{C}(42)-\mathrm{C}(52)-\mathrm{C}(62)-\mathrm{C}(102)$ & 179.2(3) \\
\hline $\mathrm{C}(22)-\mathrm{C}(12)-\mathrm{C}(62)-\mathrm{C}(52)$ & $-0.4(5)$ \\
\hline $\mathrm{N}(11)-\mathrm{C}(12)-\mathrm{C}(62)-\mathrm{C}(52)$ & $-175.7(3)$ \\
\hline $\mathrm{C}(22)-\mathrm{C}(12)-\mathrm{C}(62)-\mathrm{C}(102)$ & $-179.4(3)$ \\
\hline $\mathrm{N}(11)-\mathrm{C}(12)-\mathrm{C}(62)-\mathrm{C}(102)$ & $5.3(5)$ \\
\hline $\mathrm{C}(32)-\mathrm{C}(22)-\mathrm{C}(72)-\mathrm{C}(82)$ & $-68.5(4)$ \\
\hline $\mathrm{C}(12)-\mathrm{C}(22)-\mathrm{C}(72)-\mathrm{C}(82)$ & $109.6(4)$ \\
\hline $\mathrm{C}(32)-\mathrm{C}(22)-\mathrm{C}(72)-\mathrm{C}(92)$ & $55.7(4)$ \\
\hline $\mathrm{C}(12)-\mathrm{C}(22)-\mathrm{C}(72)-\mathrm{C}(92)$ & $-126.2(4)$ \\
\hline$C(52)-C(62)-C(102)-C(112)$ & $32.4(5)$ \\
\hline
\end{tabular}




\begin{tabular}{|c|c|}
\hline$C(12)-C(62)-C(102)-C(112)$ & $-148.6(3)$ \\
\hline$C(52)-C(62)-C(102)-C(122)$ & $-92.9(4)$ \\
\hline$C(12)-C(62)-C(102)-C(122)$ & $86.1(4)$ \\
\hline $\mathrm{C}(41)-\mathrm{N}(21)-\mathrm{C}(13)-\mathrm{C}(23)$ & $-103.1(4)$ \\
\hline $\mathrm{Fe}(01)-\mathrm{N}(21)-\mathrm{C}(13)-\mathrm{C}(23)$ & $79.4(3)$ \\
\hline $\mathrm{C}(41)-\mathrm{N}(21)-\mathrm{C}(13)-\mathrm{C}(63)$ & $79.5(4)$ \\
\hline $\mathrm{Fe}(01)-\mathrm{N}(21)-\mathrm{C}(13)-\mathrm{C}(63)$ & $-98.0(3)$ \\
\hline $\mathrm{C}(63)-\mathrm{C}(13)-\mathrm{C}(23)-\mathrm{C}(33)$ & $-3.0(5)$ \\
\hline $\mathrm{N}(21)-\mathrm{C}(13)-\mathrm{C}(23)-\mathrm{C}(33)$ & 179.6(3) \\
\hline $\mathrm{C}(63)-\mathrm{C}(13)-\mathrm{C}(23)-\mathrm{C}(73)$ & $178.7(3)$ \\
\hline $\mathrm{N}(21)-\mathrm{C}(13)-\mathrm{C}(23)-\mathrm{C}(73)$ & $1.2(5)$ \\
\hline $\mathrm{C}(13)-\mathrm{C}(23)-\mathrm{C}(33)-\mathrm{C}(43)$ & $0.0(5)$ \\
\hline $\mathrm{C}(73)-\mathrm{C}(23)-\mathrm{C}(33)-\mathrm{C}(43)$ & $178.4(4)$ \\
\hline$C(23)-C(33)-C(43)-C(53)$ & $2.6(6)$ \\
\hline $\mathrm{C}(33)-\mathrm{C}(43)-\mathrm{C}(53)-\mathrm{C}(63)$ & $-2.1(6)$ \\
\hline $\mathrm{C}(43)-\mathrm{C}(53)-\mathrm{C}(63)-\mathrm{C}(13)$ & $-0.8(5)$ \\
\hline$C(43)-C(53)-C(63)-C(103)$ & $176.5(3)$ \\
\hline $\mathrm{C}(23)-\mathrm{C}(13)-\mathrm{C}(63)-\mathrm{C}(53)$ & $3.3(5)$ \\
\hline $\mathrm{N}(21)-\mathrm{C}(13)-\mathrm{C}(63)-\mathrm{C}(53)$ & $-179.3(3)$ \\
\hline$C(23)-C(13)-C(63)-C(103)$ & $-173.9(3)$ \\
\hline $\mathrm{N}(21)-\mathrm{C}(13)-\mathrm{C}(63)-\mathrm{C}(103)$ & $3.5(5)$ \\
\hline $\mathrm{C}(33)-\mathrm{C}(23)-\mathrm{C}(73)-\mathrm{C}(93)$ & $-92.4(4)$ \\
\hline $\mathrm{C}(13)-\mathrm{C}(23)-\mathrm{C}(73)-\mathrm{C}(93)$ & $85.9(4)$ \\
\hline $\mathrm{C}(33)-\mathrm{C}(23)-\mathrm{C}(73)-\mathrm{C}(83)$ & $33.2(5)$ \\
\hline $\mathrm{C}(13)-\mathrm{C}(23)-\mathrm{C}(73)-\mathrm{C}(83)$ & $-148.5(3)$ \\
\hline $\mathrm{C}(53)-\mathrm{C}(63)-\mathrm{C}(103)-\mathrm{C}(113)$ & $-67.1(4)$ \\
\hline$C(13)-C(63)-C(103)-C(113)$ & $110.0(4)$ \\
\hline $\mathrm{C}(53)-\mathrm{C}(63)-\mathrm{C}(103)-\mathrm{C}(123)$ & $56.0(4)$ \\
\hline$C(13)-C(63)-C(103)-C(123)$ & $-126.9(4)$ \\
\hline $\mathrm{N}(21)-\mathrm{Fe}(01)-\mathrm{O}(14)-\mathrm{C}(34)$ & $119.6(2)$ \\
\hline $\mathrm{N}(11)-\mathrm{Fe}(01)-\mathrm{O}(14)-\mathrm{C}(34)$ & $-128.2(2)$ \\
\hline $\mathrm{C}(14)-\mathrm{Fe}(01)-\mathrm{O}(14)-\mathrm{C}(34)$ & $-3.0(2)$ \\
\hline $\mathrm{C}(44)-\mathrm{C}(14)-\mathrm{C}(24)-\mathrm{C}(34)$ & $174.8(3)$ \\
\hline $\mathrm{Fe}(01)-\mathrm{C}(14)-\mathrm{C}(24)-\mathrm{C}(34)$ & $-4.3(4)$ \\
\hline $\mathrm{Fe}(01)-\mathrm{O}(14)-\mathrm{C}(34)-\mathrm{C}(164)$ & $-121.3(2)$ \\
\hline $\mathrm{Fe}(01)-\mathrm{O}(14)-\mathrm{C}(34)-\mathrm{C}(24)$ & $1.4(3)$ \\
\hline $\mathrm{Fe}(01)-\mathrm{O}(14)-\mathrm{C}(34)-\mathrm{C}(104)$ & $117.4(2)$ \\
\hline
\end{tabular}




\begin{tabular}{|c|c|}
\hline $\mathrm{C}(14)-\mathrm{C}(24)-\mathrm{C}(34)-\mathrm{O}(14)$ & $2.3(4)$ \\
\hline $\mathrm{C}(14)-\mathrm{C}(24)-\mathrm{C}(34)-\mathrm{C}(164)$ & $121.3(3)$ \\
\hline $\mathrm{C}(14)-\mathrm{C}(24)-\mathrm{C}(34)-\mathrm{C}(104)$ & $-116.2(3)$ \\
\hline $\mathrm{C}(24)-\mathrm{C}(14)-\mathrm{C}(44)-\mathrm{C}(94)$ & 167.2(3) \\
\hline $\mathrm{Fe}(01)-\mathrm{C}(14)-\mathrm{C}(44)-\mathrm{C}(94)$ & $-14.0(4)$ \\
\hline $\mathrm{C}(24)-\mathrm{C}(14)-\mathrm{C}(44)-\mathrm{C}(54)$ & $-15.5(5)$ \\
\hline $\mathrm{Fe}(01)-\mathrm{C}(14)-\mathrm{C}(44)-\mathrm{C}(54)$ & $163.3(3)$ \\
\hline$C(94)-C(44)-C(54)-C(64)$ & $-2.0(5)$ \\
\hline $\mathrm{C}(14)-\mathrm{C}(44)-\mathrm{C}(54)-\mathrm{C}(64)$ & $-179.4(3)$ \\
\hline $\mathrm{C}(44)-\mathrm{C}(54)-\mathrm{C}(64)-\mathrm{C}(74)$ & $0.0(5)$ \\
\hline $\mathrm{C}(54)-\mathrm{C}(64)-\mathrm{C}(74)-\mathrm{C}(84)$ & $1.3(5)$ \\
\hline $\mathrm{C}(64)-\mathrm{C}(74)-\mathrm{C}(84)-\mathrm{C}(94)$ & $-0.6(5)$ \\
\hline$C(74)-C(84)-C(94)-C(44)$ & $-1.5(5)$ \\
\hline $\mathrm{C}(54)-\mathrm{C}(44)-\mathrm{C}(94)-\mathrm{C}(84)$ & $2.7(5)$ \\
\hline $\mathrm{C}(14)-\mathrm{C}(44)-\mathrm{C}(94)-\mathrm{C}(84)$ & $-179.8(3)$ \\
\hline $\mathrm{O}(14)-\mathrm{C}(34)-\mathrm{C}(104)-\mathrm{C}(154)$ & $162.2(3)$ \\
\hline $\mathrm{C}(164)-\mathrm{C}(34)-\mathrm{C}(104)-\mathrm{C}(154)$ & $44.0(4)$ \\
\hline $\mathrm{C}(24)-\mathrm{C}(34)-\mathrm{C}(104)-\mathrm{C}(154)$ & $-79.3(4)$ \\
\hline $\mathrm{O}(14)-\mathrm{C}(34)-\mathrm{C}(104)-\mathrm{C}(114)$ & $-20.6(4)$ \\
\hline $\mathrm{C}(164)-\mathrm{C}(34)-\mathrm{C}(104)-\mathrm{C}(114)$ & $-138.8(3)$ \\
\hline$C(24)-C(34)-C(104)-C(114)$ & $97.9(3)$ \\
\hline $\mathrm{C}(154)-\mathrm{C}(104)-\mathrm{C}(114)-\mathrm{C}(124)$ & $0.5(5)$ \\
\hline $\mathrm{C}(34)-\mathrm{C}(104)-\mathrm{C}(114)-\mathrm{C}(124)$ & $-176.8(3)$ \\
\hline$C(104)-C(114)-C(124)-C(134)$ & $-1.6(5)$ \\
\hline $\mathrm{C}(114)-\mathrm{C}(124)-\mathrm{C}(134)-\mathrm{C}(144)$ & $1.2(5)$ \\
\hline$C(124)-C(134)-C(144)-C(154)$ & $0.1(5)$ \\
\hline C(134)-C(144)-C(154)-C(104) & $-1.2(5)$ \\
\hline $\mathrm{C}(114)-\mathrm{C}(104)-\mathrm{C}(154)-\mathrm{C}(144)$ & $0.9(5)$ \\
\hline$C(34)-C(104)-C(154)-C(144)$ & 178.1(3) \\
\hline $\mathrm{O}(14)-\mathrm{C}(34)-\mathrm{C}(164)-\mathrm{C}(174)$ & $-76.1(4)$ \\
\hline$C(24)-C(34)-C(164)-C(174)$ & 163.1(3) \\
\hline $\mathrm{C}(104)-\mathrm{C}(34)-\mathrm{C}(164)-\mathrm{C}(174)$ & $43.9(4)$ \\
\hline $\mathrm{O}(14)-\mathrm{C}(34)-\mathrm{C}(164)-\mathrm{C}(214)$ & $99.3(4)$ \\
\hline$C(24)-C(34)-C(164)-C(214)$ & $-21.4(4)$ \\
\hline$C(104)-C(34)-C(164)-C(214)$ & $-140.7(3)$ \\
\hline C(214)-C(164)-C(174)-C(184) & $0.5(5)$ \\
\hline$C(34)-C(164)-C(174)-C(184)$ & 176.1(3) \\
\hline
\end{tabular}




$\begin{array}{lc}\text { C(164)-C(174)-C(184)-C(194) } & 0.0(6) \\ \text { C(174)-C(184)-C(194)-C(204) } & -0.1(6) \\ \text { C(184)-C(194)-C(204)-C(214) } & -0.3(6) \\ \text { C(174)-C(164)-C(214)-C(204) } & -0.9(6) \\ \text { C(34)-C(164)-C(214)-C(204) } & -176.4(3) \\ C(194)-C(204)-C(214)-C(164) & 0.9(6)\end{array}$




\section{References}

${ }^{1}$ George, G. N. EXAFSPAK; Stanford Synchrotron Radiation Lightsource, Stanford Linear Accelerator Center; Stanford University: Stanford, CA, 2001.

${ }^{2}$ Neese, F. WIREs Comput. Mol. Sci. 2012, 2, 73-78.

${ }^{3}$ (a) Becke, A. D. Phys. Rev. A 1988, 38, 3098-3100. (b) Perdew, J. P. Phys. Rev. B 1986, 33, 8822-8824.

4 (a) Weigend, F.; Ahlrichs, R. Phys. Chem. Chem. Phys. 2005, 7, 3297-3305. (b) Pantazis, D. A.; Chen, X.-Y.; Landis, C. R.; Neese, F. J. Chem. Theory Comput. 2008, 4, 908-919. (c) van Lenthe, E.; van der Avoird, A.; Wormer, P. E. S. J. Chem. Phys. 1998, 108, 4783-4796.

${ }^{5}$ van Wüllen, C. J. Chem. Phys. 1998, 109, 392-399.

${ }^{6}$ Neese, F. Inorg. Chim. Acta 2002, 337, 181-192.

${ }^{7}$ DeBeer George, S.; Petrenko, T.; Neese, F. J. Phys. Chem. A 2008, 112, 12936-12943.

${ }^{8}$ (a) Becke, A.D. J. Chem. Phys. 1993, 98, 5648-5652. (b) Lee, C.; Yang, W.; Parr, R. G. Phys. Rev. B 1988, 37, 785-789.

${ }^{9}$ Weigend, F.; Ahlrichs, R.. Phys. Chem. Chem. Phys. 2005, 7, 3297-3305.

${ }^{10}$ Neese, F.; Wennmohs, F.; Hansen, A.; Becker, U. Chem. Phys. 2009, 356, 98-109.

${ }^{11}$ Neese, F. J. Chem. Phys. 2003, 119, 9428-9443.

12 Neese, F. J. Am. Chem. Soc. 2006, 128, 10213-10222.

${ }^{13}$ APEX2, version 2013.2-0; Bruker AXS: Madison, WI, 2013.

${ }^{14}$ Sheldrick, G. M. SADABS, version 2012/1; University of Göttingen: Göttingen, Germany, 2012.

${ }^{15}$ SAINT, version 8.27B; Bruker AXS: Madison, WI, 2013.

${ }^{16}$ Burla, M. C.; Caliandro, R.; Camalli, M.; Carrozzini, B.; Cascarano, G. L.; Giacovazzo, C.; Mallamo, M.; Mazzone, A.; Polidori, G.; Spagna, R. SIR2011: a new package for crystal structure determination and refinement, version 1.0; Istituto di Cristallografia: Bari, Italy, 2012. ${ }^{17}$ Sheldrick, G. M. SHELXL-2013/4, University of Göttingen: Göttingen, Germany, 2013. 